Aus der Abteilung Unfallchirurgie, Plastische und Wiederherstellungschirurgie

(Prof.Dr.med.K.M.Stürmer)

im Zentrum Chirurgie

der Medizinischen Fakultät der Universität Göttingen

\title{
Oberschenkelfraktur beim Polytrauma
}

Eine retrospektive Studie auf der Grundlage des Göttinger Polytraumaregisters

\author{
INAUGURAL-DISSERTATION \\ zur Erlangung des Doktorgrades \\ der Medizinischen Fakultät \\ der Georg-August-Universität zu Göttingen
}

vorgelegt von

\section{Alexandra Böhmert}

aus Naumburg/Saale

Göttingen 2010 
Dekan: Prof. Dr.med.C.Frömmel

I. Berichterstatter: Prof.Dr.med.K.Dresing

II. Berichterstatter/in: Priv.-Doz.Dr.med.Klinger

III. Berichterstatter/in:

Tag der mündlichen Prüfung: 22.November 2010 


\section{INHALTSVERZEICHNIS}

1. Einleitung $S .1$

1.1. Definition des Polytraumas S.1

1.2. Historie über die Versorgung der Mehrfachverletzungen S.3

1.3. Epidemiologie der Unfallentwicklung $\quad \mathrm{S} .5$

1.4. Präklinisches Management des polytraumatisierten Patienten $\quad$ S.11

1.5. Klinische Versorgung des Polytraumas $\quad \mathbf{S . 1 5}$

1.6. Rehabilitation nach Polytrauma $\quad \mathbf{S . 2 5}$

1.7. Pathophysiologie des Polytraumas $\mathbf{S . 2 6}$

1.8. Frakturen der Extremitäten im Rahmen eines Polytraumas $\quad$ S.28

1.9. Osteosynthetische Verfahren für die Versorgung der Femurfraktur $\quad$ S.31

1.10. Fragestellung der Untersuchung $\mathbf{S} 33$

2. Material und Methodik $\quad \mathbf{5 . 3 4}$

2.1. Datenerfassung $\mathbf{S . 3 4}$

2.2. Einschlusskriterien $\mathbf{S . 3 4}$

2.3. Nachuntersuchung der ausgewählten Patienten $\quad \mathbf{S} 35$

2.4. Datenverarbeitung und Statistik $\quad$ S.36

2.5. Auswertung des Fragebogens und der klinischen Nachuntersuchung $\mathbf{S . 3 7}$

$\begin{array}{ll}\text { 2.6. Definitionen } & 5.37\end{array}$

$\begin{array}{ll}\text { 3. Ergebnisse } & \$ 39\end{array}$

3.1. Überblick über die Schockraumpatienten $\quad$ S.39

3.2. Gesamte Polytraumastudie $\quad \$ .40$

3.2.1. Abbreviated Injury Scale (AIS) $\quad \mathbf{4} 41$

3.3. Gesamtpolytraumastudie versus Polytraumapatienten mit $\quad \mathbf{S . 4 3}$

Femurfraktur

3.3.1. Jahresverteilung $\quad \mathrm{S.43}$

3.3.2. Geschlechtsverteilung $\quad 5.44$

3.3.3. Alters- und Geschlechtsverteilung nach Schweregradgruppierung $\quad$ S.44

3.3.4. Todeszeitpunkt $\quad \mathbf{S . 4 5}$

3.3.5. Intensivstationsaufenthalts- und Beatmungsdauer $\quad$ S.46

3.3.6. Hämoglobin und Hämatokrit $\quad \mathrm{S} .48$

3.3.7. Zielkrankenhaus $\quad$ S.49

3.3.8. Unfallursache $\quad \mathrm{S.51}$

3.4. Präklinische und klinische Daten der Polytraumapatienten mit $\mathbf{S . 5 1}$ Femurfraktur im Gruppenvergleich

3.4.1. Geschlechts- und Altersverteilung $\quad$ S.51

3.4.2. Jahresverteilung $\$ .53$

3.4.3. Schweregradverteilung nach ISS $\$ .53$

3.4.4. Zielkrankenhaus $\quad \mathbf{5 . 5 4}$

3.4.5. Unfallursache $\quad \mathbf{5} 55$

3.4.6. Verletzungsmuster $\quad$ S.55

3.4.7. Seitenverteilung der Femurfrakturen und Grad der offenen S.56 Frakturen

3.4.8. AO-Klassifikation $\quad$ S.57

3.4.9. Primär- und Sekundär-Operation zur Frakturversorgung am Femur $\quad S .57$ 
3.4.10. Intensivstationsaufenthalts- und Beatmungsdauer $\quad$ S.59

3.4.11. Hämoglobin und Hämatokrit $\quad S .60$

3.4.12. Stationäre Aufenthaltsdauer und Entlassung S.61

3.4.13. Komplikationen und Risikofaktoren $\quad$ S.63

3.5. Spätergebnisse $\quad$ S.64

3.5.1. Auswertung des Fragebogens $\quad$ S.65

3.5.1.1. Anzahl der Patienten und Nachuntersuchungszeitraum $\quad$ S.65

3.5.1.2. Soziodemographische Daten $\quad$ S.65

3.5.1.3. Veränderungen im privaten Umfeld und Lebensumstände S.68

3.5.1.4. Aussagen zu Schmerzen nach dem Trauma $\quad$ S.70

3.5.1.5. Belastbarkeit und Gehstrecke nach dem Trauma $\quad$ S.75

3.5.2. Auswertung der klinischen Nachuntersuchung $\quad$ S.77

$\begin{array}{lll}\text { 3.5.2.1. Altersverteilung zum Untersuchungszeitpunkt } & \text { S.77 }\end{array}$

3.5.2.2. Bodymassindex (BMI) der untersuchten Patienten $\quad$ S.78

3.5.2.3. Gangbild und Hilfsmittelnutzung $\quad$ S.78

$\begin{array}{ll}\text { 3.5.2.4. Differenzierte Standarten } & \text { S.79 }\end{array}$

3.5.2.5. Schmerzlokalisation und Schmerzart $\quad$ S.80

3.5.2.6. Längen- und Umfangsbestimmungen S.81

3.5.2.7. Bestimmung der Bewegungsausmaße der angrenzenden S.84

3.5.2.8. Modifizierter Score nach NEER et al. (1967) S.85

$\begin{array}{ll}\text { 4. Diskussion } & 5.87\end{array}$

5. Zusammenfassung $\quad \$ .98$

5.1. Einleitung $\quad S .98$

5.2. Methodik $\quad S .98$

5.3. Ergebnisse $\quad \mathrm{S.98}$

$\begin{array}{ll}\text { 5.4. Schlussfolgerung } & \text { S.99 }\end{array}$

6. Anhang $\quad 5.101$

6.1. Glasgow-Coma-Scale $\quad$ S.101

6.2. Anforderungen an Krankenhäuser zur Behandlung von $\quad \mathrm{S.102}$

Schwerverletzten im Rahmen des Traumanetzwerkes

6.3. Mangled Extremity Severity Score (MESS) $\quad 5.104$

6.4. Modifizierter Score nach NEER et al. (1967) $\quad$ S.105

6.5. AO-Klassifikation der Femurfraktur $\quad \$ .106$

6.6. Anschreiben an die Patienten $\quad 5.109$

6.7. Fragebogen $\quad \$ .110$

$\begin{array}{ll}\text { 6.8. Nachuntersuchungsbogen } & \text { S.116 }\end{array}$

$\begin{array}{ll}\text { 7. Literaturverzeichnis } & \$ 119\end{array}$

$\begin{array}{ll}\text { 8. Abkürzungsverzeichnis } & \mathbf{S . 1 2 7}\end{array}$ 


\section{Einleitung}

\section{Einleitung}

Die Behandlung von Unfallverletzten gehört zu den ältesten Aufgaben der Medizin. „In den zurückliegenden Jahrhunderten hatte sich der Mensch mit der natürlichen Umwelt oder seiner eigenen Gattung im Kampf mit primitiven Waffen auseinander zu setzen. Entsprechend niedrig war die Zahl der Unfälle. Die Traumatisierung entsprach den Verletzungen durch Tiere, wie durch Schnitt und Stichwunden im Kampf. Die Behandlungsmethoden waren primitiv. Die Zahl der tödlichen Verluste durch diese Verletzungen wie infolge von Infektionen und auch die Anzahl der Defektheilungen war groß, erreichte aber, trotz fehlender Statistiken, wohl niemals die heutigen Zahlen.“ (REHN 1973, S.432)

\subsection{Definition des Polytraumas}

„Der Begriff "Polytrauma" beschreibt die Verletzung einer oder mehrerer Körperregionen oder Organsysteme, wobei die Einzelverletzungen überlebbar sind, die Kombination der Einzelverletzungen jedoch durch die nachfolgende systemische Inflammationsreaktion tödlich enden kann." (LEHNERT und MARZI 2009, S.386)

SCHWEIBERER et al. (1987) verstehen unter Polytrauma die gleichzeitig entstandene schwere Verletzung in mindestens zwei der vier Körperregionen - Kopf, Thorax, Abdomen und Bewegungsapparat - bzw. eine besonders schwere Mehrfachverletzung des Bewegungsapparates (Wirbelsäule, Becken, Extremitäten), wobei ein Bereich so schwer geschädigt ist, dass eine vitale Bedrohung besteht.

Ähnlich lautet auch die Definition der Mehrfachverletzung von TSCHERNE et al. (1984), bei der die Verletzungen mehrerer Körperregionen oder Organsysteme ebenfalls gleichzeitig entstanden sind, wobei wenigstens eine Verletzung oder die Kombination mehrerer lebensbedrohlich ist.

Diese Definition wurde durch SCHWEIBERER et al. (1987) erweitert und präzisiert. Nach ihrer Definition gelten unter anderem auch schwere Verletzungen der Region Bewegungsapparat (Wirbelsäule, Becken, Extremitäten) sowie 2- und 3-gradige Verbrennungen mit mehr als $10 \%$ betroffener Körperoberfläche als Polytrauma. 


\section{Einleitung}

Nach RUCHHOLTZ et al. (1996) treten bei Polytraumatisierten zu 61\% Verletzungen des Kopfes, zu 63\% des Thorax, zu 43\% des Abdomens und zu 56\% Verletzungen des Bewegungsapparates auf.

In der Anfangsphase ist der Schweregrad des Polytraumas nicht immer an der Summe der Einzelverletzungen oder an hämodynamischen Parametern erkennbar. Aus diesem Grunde werden verschiedene Scores zur Schweregradklassifikation angewandt, die der frühzeitigen Identifizierung Schwerverletzter dienen sollen und Hilfe bei der Entscheidung über notwendige therapeutische Maßnahmen geben können. Die große Vielfalt der zur Verfügung stehenden Bewertungssysteme weist jedoch darauf hin, dass es einige Schwierigkeiten mit sich bringt, sie zu entwickeln. Sie sollen einerseits objektiv, aussagekräftig und verlässlich, andererseits aber auch praktikabel sein (SEEFELDER et al. 1988).

Einige orientieren sich an anatomischen Gesichtspunkten wie z.B. die Abbreviated Injury Scale - AIS (Committee on Injury Scaling) und der Injury Severity Score - ISS (BAKER et al. 1974). Andere wiederum basieren auf physiologischen Veränderungen wie der Akute Trauma Index (MILHOLLAND et al. 1979), der Trauma Score (CHAMPION et al. 1981) und der Revised Trauma Score (CHAMPION et al. 1989).

Der Hannoversche Polytraumaschlüssel (PTS) nach TSCHERNE et al. (1984), der 1991 nochmals überarbeitet wurde, beinhaltet anatomische Gesichtspunkte sowie das Alter des Patienten.

Die Bewertungssysteme sollten zum einen gefährdete Patienten herausstellen (Sensitivität) und zum anderen aber auch die Verletzungsschwere mäßig Gefährdeter nicht überbewerten (Spezifität).

Nach einer Dissertation von BROSS (1995) betrug bei seiner Untersuchung die Spezifität für den ISS 92,1\% und für den PTS 96,5\%, die Sensitivität betrug $71 \%$ für den ISS und $12,9 \%$ für den PTS.

Der ISS ist einer der am weitesten verbreiteten Traumascores (DEANE et al. 1986). Der einzige Kritikpunkt an diesem Score ist, dass er das Patientenalter nicht berücksichtigt. 


\section{Einleitung}

Die Schwächen des PTS liegen in der zu hohen Bewertung der ExtremitätenVerletzungen und in der zu geringen Bewertung der Schädel-Hirn-Verletzungen.

Wägt man nun die Vor- und Nachteile beider Score-Systeme ab, so scheint der ISS besser mit dem klinischen Verlauf zu korrelieren (BROSS 1995).

Deshalb wird an der Universitätsmedizin Göttingen zur Beschreibung von Polytraumatisierten der AIS in Verbindung mit dem ISS angewandt. Der praktische Nutzen der Scores ist jedoch umstritten, weil ihre Berechnung im allgemeinen erst nach Abschluss der Diagnostik möglich ist. Bedeutsam sind sie aber für die einheitliche Schweregradbeurteilung, die die Voraussetzung für eine Vergleichbarkeit von Daten, zum Beispiel Therapieergebnissen unterschiedlicher Einrichtungen, ermöglicht.

\subsection{Historie über die Versorgung der Mehrfachverletzungen}

Der Begriff Mehrfachverletzung war bereits zu der Zeit der Trojanischen Kriege (1200 v. Chr.) bekannt, denn Homer berichtet in seiner Ilias, dass die „Schwerverletzten Helden“ unter einen Baum zum Sterben gelegt worden seien. Zu dieser Zeit lag die Letalität weit über 80\%, auch zur Zeit der Markomannischen Kriege (167-75 v.Chr.) lag sie immer noch über $70 \%$, obwohl man im Römischen Reich eine Organisation zur Unfallversorgung gegründet hatte.

In der Chirurgia Magna wurde 1363 entschieden, dass man die Polytraumatisierten nicht länger durch „nutzloses Hantieren“ quälen sollte.

Zum Zeitpunkt der Napoleonischen Kriege (1792ff.) erzielte man erstmals eine Verbesserung in der Behandlung von Mehrfachverletzten, nachdem J.D.Larrey (erster Heereschirurg Napoleons) „die Folgen des traumatischen Schocks und die Bedeutung der Akutversorgung dieser Patienten“ erkannt hatte (TSCHERNE et al. 1987).

Erst im 20.Jahrhundert hatte sich dann ein grundsätzlicher Wandel der Behandlung von Polytraumatisierten vollzogen. Nachdem 1922 "Polytrauma" nur ein Begriff, aber noch kein Thema für die Deutsche Gesellschaft für Chirurgie war und man die Behandlung von Schwerstverletzten als für einen „besseren Chirurgen für unwürdig“ 


\section{Einleitung}

hielt, wurde 1922 die Gesellschaft für Unfallheilkunde gegründet (TSCHERNE et al. 1988).

Durch das geänderte Anspruchsdenken der Gesellschaft im Bezug auf die Wiederherstellung von Unfallfolgen und immer neuere Erkenntnisse in den Grundlagenfächern, wie Physiologie und Biochemie, konnte sich die Behandlung von Polytrauma-Patienten entscheidend fortentwickeln.

Seit den 20er Jahren lassen sich bedeutende Fortschritte in der Versorgung von Mehrfachverletzten nachweisen, die nicht nur durch das Fach Chirurgie begründet wurden, sondern vielmehr auch durch Fortschritte in den Forschungsgebieten wie Physiologie, Biochemie oder auch Pharmakologie. Durch die Erkenntnisse über die pathophysiologischen Veränderungen beim Schock konnte man sie besser mittels Intensivmedizin behandeln. Da die Patienten nunmehr die ersten Tage nach dem Trauma überlebten, konnte es jetzt vermehrt auch zu den Schockfolgeerkrankungen wie das Nieren- oder Lungenversagen kommen.

Während die klassischen Komplikationen wie Schock, akutes Nierenversagen (ANV) und auch das Lungenversagen (ARDS) inzwischen durch optimierte präklinische Versorgung und verbesserte Intensivmedizin weitgehend vermieden werden können, ist das Multiorganversagen (MOV) zu einem limitierenden Faktor des chirurgischen Erfolgs geworden (DEITCH 1992).

CRUMP et al. (1988) sprechen von einem iatrogenen Problem, hervorgerufen durch die Tatsache, dass durch die moderne Medizin heute die Patienten lange genug überleben, damit die dem Organversagen zugrundeliegenden Pathomechanismen erst wirken können.

Diese Entwicklung lässt sich auch in nachfolgender Abbildung 1 über die historischen Veränderungen der Todesursachen nach Polytrauma erkennen. 


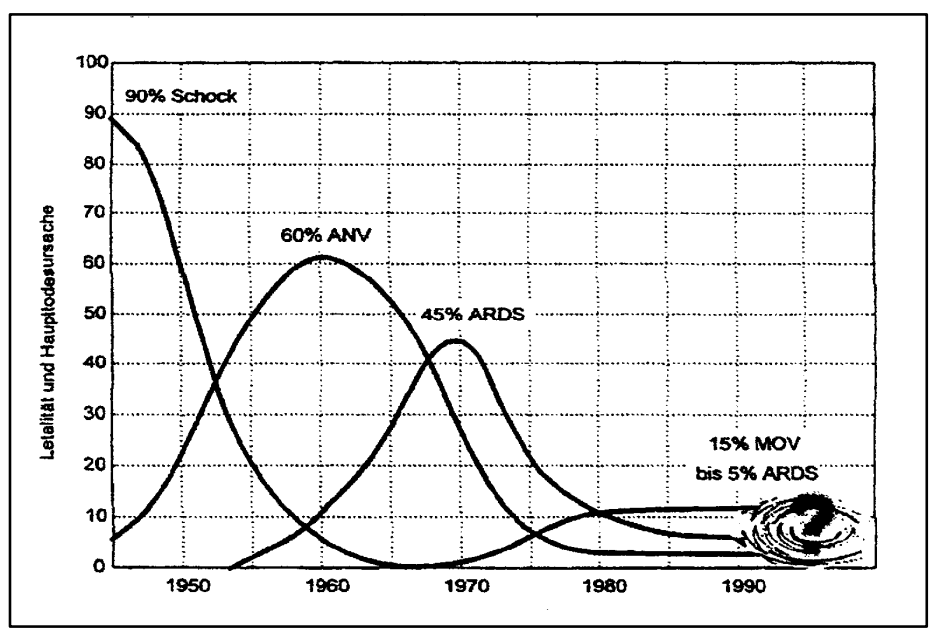

Abbildung 1: Historische Veränderungen der Todesursachen nach schwerem Trauma (nach TSCHERNE und REGEL 1997, S.220)

Heute kann man die verstorbenen Patienten fast alle in zwei Kategorien einteilen, zum einen die Gruppe der Patienten, die an Verletzungen verstorben sind, die nicht mit dem Leben vereinbar sind, und zum anderen die Gruppe der Patienten, die nach Tagen bis Wochen nach dem Trauma vorwiegend an konsekutivem Organversagen mit oftmals septiformen Begleitumständen versterben. Dieser als „späte Tod“ bezeichnete Vorgang steht nicht mehr in direktem Zusammenhang mit dem Trauma, dennoch ist seine Pathogenese umstritten und auch die Therapie sehr unspezifisch (NEUHOF 1991, SCHLAG und REDL 1988).

\subsection{Epidemiologie der Unfallentwicklung}

Das Polytrauma ist einer Epidemie unserer Zeit gleichzusetzen, es spielt die Rolle von Seuchen der vergangenen Jahrhunderte. Weltweit wird nach Untersuchungen der Weltgesundheitsorganisation (WHO) die Bedeutung des Traumas weiter ansteigen bedingt durch die technische Entwicklung in den Ländern der Dritten Welt (HAVEMANN 1972).

Sucht man nach den Ursachen für Polytraumen, so findet man in der Literatur unterschiedliche Angaben. So wird der Anteil der durch Verkehrsunfall verursachten Polytraumen zwischen $50-80 \%$ beschrieben. Die Angaben differieren mehr oder 


\section{Einleitung}

weniger durch unterschiedliche definitorische Ansätze. Es wird bereits seit mehreren Jahren diskutiert, inwieweit die Arbeitswegeunfälle den Arbeitsunfällen oder aber den Verkehrsunfällen zugeordnet werden. Der Arbeitsunfall bzw. der Sturz aus großer Höhe stehen an 2. Stelle der Ursachen für Polytraumen.

Tabelle 1: Unfallursachen aus verschiedenen Untersuchungen.

\begin{tabular}{|c|c|c|c|c|c|c|c|}
\hline Autor & $\begin{array}{l}\text { Verkehrs- } \\
\text { unfall }\end{array}$ & $\begin{array}{l}\text { Arbeits- } \\
\text { unfall }\end{array}$ & Suizid & $\begin{array}{l}\text { Freizeit- } \\
\text { unfall }\end{array}$ & Haus- & $\begin{array}{l}\text { Sturz } \\
\text { aus }>3 m\end{array}$ & Sonstiges \\
\hline Dittel/Weller 1981 & $77 \%$ & $19 \%$ & $1,3 \%$ & $1,30 \%$ & & & $1,40 \%$ \\
\hline Dittmer et al. 1983 & $81,60 \%$ & $6,60 \%$ & $5,2 \%$ & $3,30 \%$ & $3 \%$ & & $0,30 \%$ \\
\hline Zenker et al. 1992 & $82,90 \%$ & & & & & $13,50 \%$ & $3,60 \%$ \\
\hline Haas et al. 1997 & $70 \%$ & $10 \%$ & & $10 \%$ & $10 \%$ & & \\
\hline Bernhard et al. 2004 & $55 \%$ & $24 \%$ & & & & $14 \%$ & \\
\hline
\end{tabular}

Laut Datenerfassung des Statistischen Bundesamtes (Abrufdatum 10.08.2009) wurden zum Beispiel im Jahr 2008 bundesweit insgesamt 2,29 Mio. Unfälle polizeilich aufgenommen, das waren 1,8\% weniger im Vergleich zum Vorjahr. Ebenfalls rückläufig zeigte sich im Jahr 2008 die Zahl der Unfälle mit Personenschaden mit 5,2\% und getöteten Personen mit 9,5\% im Vergleich zum Vorjahr. Im Vergleich hierzu wurde lediglich im Jahr 2004 ein Rückgang der Verkehrstoten nach der Wiedervereinigung Deutschlands mit $11,7 \%$ erzielt.

Überblickt man den gesamten Zeitraum seit 1953 (seit diesem Jahr liegen erstmals Zahlen für Verkehrstote nach dem heutigen Gebietsstand vor), zeigt sich, dass ein Höchststand im Jahre 1970 gezählt wurde, wobei es 21332 Verkehrstote bei einem Kraftfahrzeugbestand von 20,8Mio. gab. Seitdem ist die Zahl der Verkehrstoten fast kontinuierlich gesunken, mit Ausnahme der ersten zwei Jahre nach dem Mauerfall. In den neuen Bundesländern stieg damals die Zahl der Toten im Verkehr vor allem durch eine gesteigerte Motorisierung sowie schlechte Straßenverhältnisse. Im Jahr 1991 erfasste man nach der Wiedervereinigung die höchste Zahl der Verkehrstoten mit 11 300. Während die Zahl der Motorisierung weiterhin stieg, sank die Zahl der Getöteten im Jahr 2008 verglichen mit 1970 um fast 80\%, die Zahl der Verletzten um 29\%, dies zeigt eine Zunahme der Verkehrssicherheit (Statistisches Bundesamt (Abrufdatum 10.08.2009)).

In der nachfolgenden Tabelle 2 ist dieser Verlauf in den letzten 50 Jahren aufgeführt, die Gründe für die Entwicklung sind sehr vielfältig, neue Verkehrsrechtliche 
Regelungen (Helmtragepflicht, Gurtanlagepflicht, Höchstgrenze für Blutalkohol, etc.) sowie verbesserte medizinische Erstversorgung am Unfallort und Versorgung in speziellen Zentren.

Tabelle 2: Straßenverkehrsunfälle, Verunglückte und Bestand an motorisierten Fahrzeugen in Deutschland (Statistisches Bundesamt (Abrufdatum 10.08.2009)).

\begin{tabular}{|c|l|l|l|l|l|}
\hline Jahr & $\begin{array}{l}\text { Unfälle mit } \\
\text { Personen- } \\
\text { schaden }\end{array}$ & \multicolumn{2}{|l|}{$\begin{array}{l}\text { Verunglückte } \\
\text { Insgesamt }\end{array}$} & $\begin{array}{l}\text { Bestand an } \\
\text { darunter } \\
\text { Gotortete } \\
\text { Fahrierten } \\
\text { in Mio. }\end{array}$ & $\begin{array}{l}\text { Getötete } \\
\text { je 10 000 } \\
\text { des } \\
\text { Bestandes }\end{array}$ \\
\hline 1953 & - & 345019 & 12631 & 4,8 & 26,5 \\
\hline 1960 & - & 518793 & 16477 & 11,6 & 14,2 \\
\hline 1970 & 414362 & 599364 & 21332 & 20,8 & 10,2 \\
\hline 1980 & 412672 & 555966 & 15050 & 33,8 & 4,4 \\
\hline 1990 & 389350 & 521977 & 11046 & 43,6 & 2,5 \\
\hline 2000 & 382949 & 511577 & 7503 & 47,5 & 1,6 \\
\hline 2003 & 354534 & 468783 & 6613 & 49,1 & 1,3 \\
\hline 2004 & 339310 & 445968 & 5842 & 49,6 & 1,2 \\
\hline 2005 & 336619 & 438804 & 5361 & 50,0 & 1,1 \\
\hline 2006 & 327984 & 427428 & 5091 & 50,3 & 1,0 \\
\hline 2007 & 335845 & 436368 & 4949 & 50,9 & 1,0 \\
\hline 2008 & 320614 & 413524 & 4477 & 51,3 & 0,9 \\
\hline
\end{tabular}

Betrachtet man die Verteilung der Unfallverletzten und Getöteten auf Bundesländerebene, zeigt sich auch hier bis auf einzelne Ausnahmen ein Rückgang im Vergleich zu den Vorjahren. Die Zahl der Verunglückten im Jahr 2008 ist mit -11\% am stärksten rückläufig im Vergleich zum Vorjahr in Brandenburg, gefolgt von Thüringen mit $-8,8 \%$ und Baden-Württemberg mit -7,9\%. Mehr Verunfallte gab es jedoch in den Stadtstaaten Bremen $+4 \%$, Berlin $+2,2 \%$ und in Hamburg $+1,2 \%$. Auch die Entwicklung der Getöteten zeigt auf Bundeslandebene eine positive Entwicklung, so zeigte sich in Bremen ein Rückgang von $-37 \%$ im Jahre 2008 und im Saarland $-30 \%$ sowie in Bayern um 21\%. Ein Anstieg der tödlich Verletzten zeigte sich im Jahr 2008 in Hamburg mit $+33 \%$, in Sachsen-Anhalt mit $+4,7 \%$ sowie in Rheinlandpfalz mit $+3,2 \%$ (Statistisches Bundesamt (Abrufdatum 10.08.2009)).

Bezogen auf je 100000 Einwohner in Deutschland verunglückten im Jahr 2008 503 Personen, das größte bevölkerungsbezogene Risiko im Straßenverkehr zu verunfallen, bestand in Hamburg mit 606/100 000 Einwohner, gefolgt vom Saarland mit 578/100 000 Einwohner und Bayern mit 574/100 000 Einwohner. Im Durchschnitt 


\section{Einleitung}

wurden 54 Personen je eine Mio. Einwohner deutschlandweit bei Straßenverkehrsunfällen getötet. Mit 92 Todesopfern je eine Mio. Einwohner lag die Gefahr tödlich zu verunglücken in Sachsen-Anhalt am höchsten, im Vergleich hierzu lagen NordrheinWestfalen mit 39 und das Saarland mit 41 Getöteten je eine Mio. Einwohner unter dem deutschen Durchschnitt.

Rückblickend zeigt sich, dass in den neuen Bundesländern große Fortschritte gemacht wurden. So gab es im Jahr 1991 noch durchschnittlich 250 Verkehrstote je eine Mio. Einwohner in den fünf neuen Bundesländern, im Jahre 2008 nur noch 76, das heißt einen Rückgang um 69\% (Statistisches Bundesamt (Abrufdatum 10.08.2009)).

Tabelle 3: Verunglückte/Getötete bei Straßenverkehrsunfällen 2001, 2007 und 2008 nach Bundesländern aufgeführt (Statistisches Bundesamt (Abrufdatum 10.08.2009)).

\begin{tabular}{|c|c|c|c|c|c|c|}
\hline Land & $\begin{array}{l}2001 \\
\text { Anzahl } \\
\text { Getötete }\end{array}$ & $\begin{array}{l}\text { Anzahl } \\
\text { Verletzte }\end{array}$ & $\begin{array}{l}2007 \\
\text { Anzahl } \\
\text { Getötete }\end{array}$ & $\begin{array}{l}\text { Anzahl } \\
\text { Verletzte }\end{array}$ & $\begin{array}{l}2008 \\
\text { Anzahl } \\
\text { Getötete }\end{array}$ & $\begin{array}{l}\text { Anzahl } \\
\text { Verletzte }\end{array}$ \\
\hline $\begin{array}{l}\text { Deutschland } \\
\text { gesamt }\end{array}$ & 6977 & 494775 & 4970 & 436474 & 4477 & 409047 \\
\hline $\begin{array}{l}\text { Baden- } \\
\text { Württemberg }\end{array}$ & 842 & 58358 & 622 & 53804 & 551 & 49105 \\
\hline Bayern & 1324 & 88409 & 992 & 75508 & 788 & 71110 \\
\hline Berlin & 65 & 17848 & 56 & 17306 & 55 & 17630 \\
\hline Brandenburg & 375 & 16834 & 263 & 12189 & 222 & 10669 \\
\hline Bremen & 19 & 4041 & 19 & 3732 & 12 & 3763 \\
\hline Hamburg & 56 & 12248 & 30 & 10595 & 40 & 10685 \\
\hline Hessen & 516 & 36350 & 373 & 32909 & 318 & 30088 \\
\hline $\begin{array}{l}\text { Mecklenburg- } \\
\text { Vorpommern }\end{array}$ & 296 & 12049 & 145 & 8806 & 132 & 8200 \\
\hline Niedersachsen & 811 & 51848 & 648 & 47176 & 595 & 44017 \\
\hline $\begin{array}{l}\text { Nordrhein- } \\
\text { Westfalen }\end{array}$ & 1038 & 93013 & 729 & 84821 & 694 & 80148 \\
\hline $\begin{array}{l}\text { Rheinland- } \\
\text { Pfalz }\end{array}$ & 313 & 24903 & 220 & 21757 & 227 & 20854 \\
\hline Saarland & 70 & 6801 & 61 & 6288 & 42 & 5952 \\
\hline Sachsen & 409 & 24128 & 236 & 20327 & 243 & 18805 \\
\hline $\begin{array}{l}\text { Sachsen- } \\
\text { Anhalt }\end{array}$ & 323 & 14985 & 213 & 13273 & 223 & 12079 \\
\hline $\begin{array}{l}\text { Schleswig- } \\
\text { Holstein }\end{array}$ & 226 & 18325 & 156 & 16428 & 149 & 15704 \\
\hline Thüringen & 294 & 14635 & 187 & 11427 & 185 & 10238 \\
\hline
\end{tabular}




\section{Einleitung}

Die am meisten gefährdeten Verkehrsteilnehmer sind die Kinder, dies ist begründet durch die mangelnde Erfahrung und ein geringeres Reaktionsvermögen. Im Jahr 2008 verunfallten insgesamt 31648 Kinder in Deutschland, wovon 102 verstarben, im Vergleich zum Vorjahr verunglückten $6,4 \%$ und mit 8,1\% verstarben auch weniger. Betrachtet man die letzten 30 Jahre, so bestätigt sich diese positive Tendenz, wenn auch die Zahlen immer noch relativ hoch sind. Im Jahr 1978 verunfallten mehr als doppelt so viele Kinder (<15Lj.) im Straßenverkehr (72 129) wie im Jahr 2008 (Statistisches Bundesamt (Abrufdatum 10.08.2009)).

Die Altersstruktur zeigt, dass der größte Anteil der Verunfallten zwischen 18 und 24 Jahren alt ist. Im Jahr 2008 verunglückten 81442 junge Erwachsene im Straßenverkehr, 71\% hiervon fuhren in einem PKW. Von 887 getöteten 18- bis 24Jährigen kamen 653 bei einem PKW-Unfall um (Statistisches Bundesamt (Abrufdatum 10.08.2009)).

Im Fokus der Verkehrssicherheitsexperten stehen in zunehmendem Maße die Senioren, zum einen stieg die Bevölkerungszahl der über 65-Jährigen in den letzten 15 Jahren stärker an als die der anderen Altersgruppen, zum anderen sind die älteren Menschen deutlich mobiler wie frühere Generationen. Im Jahr 2008 verunfallten 44527 Personen im Alter von 65 und mehr Jahren im Straßenverkehr, 45\% hiervon bei einem PKW-Unfall und 28\% als Fahrradfahrer (Statistisches Bundesamt (Abrufdatum 10.08.2009)).

Auch in den Ländern der Europäischen Union (EU) finden sich ähnliche Ansätze. Insgesamt kamen in der Europäischen Union im Jahr 2007 ca. 42500 Menschen bei Straßenverkehrsunfällen ums Leben. Im Durchschnitt wurden 86 Personen je eine Mio. Einwohner im Straßenverkehr in allen Ländern der EU tödlich verletzt. Gemessen an der Einwohnerzahl hatte Malta mit 34, gefolgt von Niederlanden mit 43 und dem Vereinigten Königreich mit 50 die wenigsten Getöteten. Im Vergleich hierzu wurden in der EU 2001 rund 54300 Personen im Straßenverkehr getötet. Ziel der EU war eine Reduzierung der Verkehrstoten bis 2010 im Vergleich zu 2001 um die Hälfte, im gesamteuropäischen Raum wurde dies bisher nicht erreicht (Statistisches Bundesamt (Abrufdatum 10.08.2009)). 


\section{Einleitung}

Sieht man diese Zahlen, so fragt man sich natürlich auch nach den Kosten, die durch Polytraumen auf die Bundesrepublik zu kommen. Da es sich hauptsächlich um junge Menschen handelt („mehr als 50\% der Verunfallten sind jünger als 40 Jahre“ (TSCHERNE et al. 1997), führt es zu enormen volkswirtschaftlichen Verlusten, sowie zu erheblichen medizinischen und psychosozialen Problemen, wie zum Beispiel Behinderung und spätere Schwierigkeiten bei der Reintegration. Dabei verursachen die Krankenhauskosten lediglich 5\% der Gesamtkosten, die Hauptkosten aus volkswirtschaftlicher Sicht werden durch Therapiefolgekosten (Rehabilitation), Rentenzahlungen sowie langfristigen Produktionsverlust verursacht (HÄUSLER et al. 2001). In einer Studie auf der Grundlage des Traumaregisters errechnete OESTERN (2008) die Krankenhausgesamtkosten mit durchschnittlich $32166 \pm 25404 €$, wobei die Behandlungskosten für die Überlebenden im Vergleich zum Gesamtkollektiv etwas höher lagen (34 $142 \pm 25697 €$ ) und die Kosten für die verstorbenen Patienten dementsprechend etwas niedriger lagen (30 $047 \pm 24383 €$ ). Hierbei fallen ca. $17676 €$ als hohe Kosten für den Intensivstationären Aufenthalt an, gefolgt von den Operationen mit ca. $7774 €$ und dem Aufenthalt auf der Normalstation mit ca. $3409 €$. In der Studie zeigte sich eine Abhängigkeit der Kosten von der Schwere der Verletzung, so stiegen die Behandlungskosten signifikant mit steigender Verletzungsschwere an. Im Vergleich zu den Studien aus den 90er Jahren, wie z.B. VARNEY et al. 1994, RUCHHOLTZ et al. 1995 und SCHMELZ et al. 2002, zeigen sich sehr verschiedene Kostenangaben, wobei zu bemerken ist, dass in den einzelnen Studien die Intensivliegedauer und Verwaltungskosten sehr unterschiedlich mit einbezogen wurden.

Ein großes Problem in der Vergütung der Versorgung Schwerstverletzter stellt immer noch die Vergütung nach G-DRG-System ("German-Diagnosis Related Groups" dar. Verschiedene Kostenanalysen von PAPE et al. (2003), RUCHHOLTZ (2004) und GROTZ et al. (2004) fanden bei der Polytrauma-Vergütung eine Unterdeckung des G-DRG-Systems von 7599 bis $12000 €$. Laut KÜHNE et al. (2006) wird eine chronische Unterdeckung der Kosten dazu führen, dass einzelne Kliniken sich aus der Behandlung von Polytraumatisierten zurückziehen bzw. auf eine Minimum reduzieren werden, was wahrscheinlich wieder eine Verschlechterung der Versorgung zur Folge haben wird. 


\section{Einleitung}

Hinzu kommen sozioökonomische Auswirkungen von Unfällen auf das Bruttosozialprodukt. Im Jahr 2004 verursachten Unfälle 56,97 Mio. Arbeitsunfähigkeitstage, dies entsprach einem Produktionsausfall von 5,2 Mrd. Euro. Nicht enthalten sind hierbei noch Kosten für Rehabilitationsmaßnahmen sowie Rentenansprüche (Bundesministeriums für Arbeit: Jahresbericht 2004, Seite 28).

\subsection{Präklinisches Management des polytraumatisierten Patienten}

Die Polytraumaversorgung stellt heute höchste Anforderungen in personeller, operativer und organisatorischer Hinsicht. Durch den Einsatz flächendeckender Rettungssysteme soll bereits durch das präklinische Rettungsteam über die geeignete Zielklinik - entsprechend der dort vorhandenen diagnostischen und therapeutischen Möglichkeiten - entschieden werden.

Die Rettung von Unfallopfern wird vorwiegend von gemeinnützig organisierten Rettungsorganisationen und der Freiwilligen Feuerwehr personell und materiell verwirklicht. Für die bodengebundene Rettung stehen der RTW (Rettungstransportwagen), der NAW (Notarztwagen), sowie das NEF (Notarzteinsatzfahrzeug) zur Verfügung. Die flächendeckende Luftrettung wird durch die regional stationierten Rettungshubschrauber (RTH) gewährleistet. Koordiniert wird die Unfallrettung durch die Rettungsleitstellen, bei denen die Notrufe eingehen. Die flächendeckende Notarztversorgung, die wiederum über die Rettungsleitstellen koordiniert wird, stellt auch eine Besonderheit des Deutschen Rettungssystems dar.

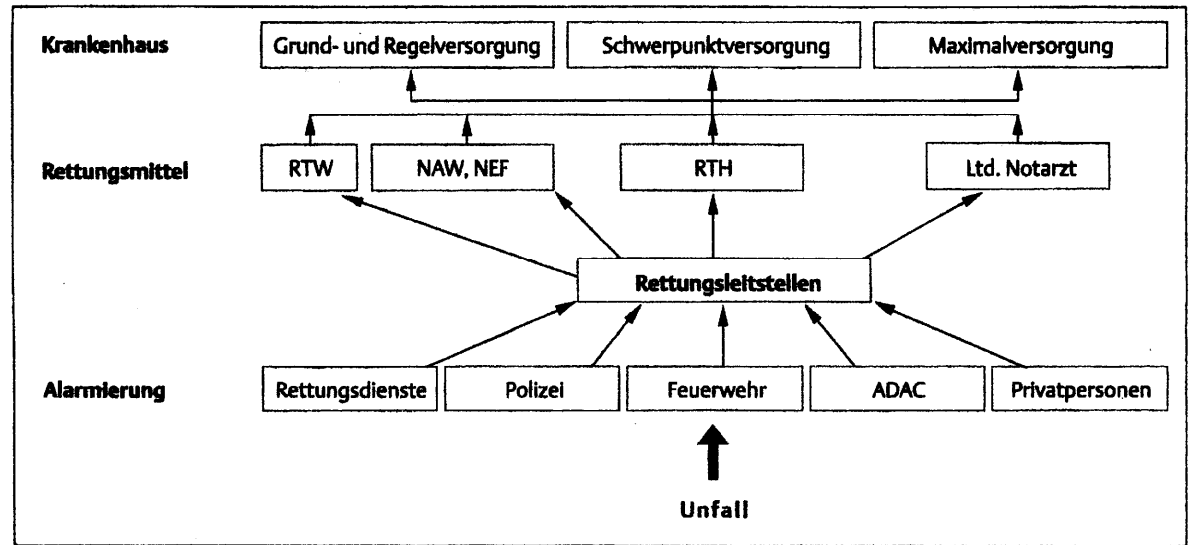

Abbildung 2: Organisation der Rettungskette. 


\section{Einleitung}

Eine flächendeckende Versorgung durch NAW- und RTH-Standorte ist bundesweit sehr unterschiedlich verwirklicht. Laut KÜHNE et al. (2006) fanden sich im Jahr 2000 im gesamten Bundesgebiet 51 Rettungshubschrauberstandorte und 1051 bodengebundene Notarzt-Standorte. Vergleicht man die einzelnen Bundesländer, so zeigt sich, dass Brandenburg, Mecklenburg-Vorpommern, Thüringen und SachsenAnhalt die größten Flächen pro NAW-Standort zu versorgen hatten, siehe hierzu auch nachfolgende Tabelle.

Tabelle 4: Versorgungsflächen von Krankenhäusern, Notarztstandorten und Luftrettungsstützpunkten (KÜHNE et al. 2006,S.362).

\begin{tabular}{|l|r|r|r|}
\hline Land & $\begin{array}{l}\text { Versorgungsfäche } \\
\left(\mathrm{km}^{2}\right) / \text { Krankenhaus }\end{array}$ & $\begin{array}{l}\text { Versorgungsfläche } \\
\left(\mathrm{km}^{2}\right) / \text { Notarztstandort }\end{array}$ & $\begin{array}{l}\text { Versorgungsfläche } \\
\left(\mathrm{km}^{2}\right) / \mathrm{RTH}\end{array}$ \\
\hline $\begin{array}{l}\text { Mecklenburg- } \\
\text { Vorpommern }\end{array}$ & 3862 & 446 & 7724 \\
\hline Brandenburg & 3684 & 526 & 9825 \\
\hline Thüringen & 1244 & 385 & 5390 \\
\hline Sachsen-Anhalt & 1572 & 417 & 20445 \\
\hline Niedersachsen & 1700 & Ka & 9523 \\
\hline Sachsen & 1227 & 239 & 6137 \\
\hline Bayern & 1356 & 346 & 8818 \\
\hline Rheinland-Pfalz & 1417 & 265 & 6615 \\
\hline $\begin{array}{l}\text { Baden- } \\
\text { Württemberg }\end{array}$ & 1021 & 273 & 7150 \\
\hline Hessen & 1242 & 330 & 7038 \\
\hline Saarland & 642 & 171 & 2568 \\
\hline $\begin{array}{l}\text { Schleswig- } \\
\text { Holstein }\end{array}$ & 1751 & 426 & 7881 \\
\hline $\begin{array}{l}\text { Nordrhein- } \\
\text { Westfalen }\end{array}$ & 420 & 160 & 4869 \\
\hline Bremen & 81 & 67 & 404 \\
\hline Berlin & 89 & 59 & 387 \\
\hline Hamburg & 108 & 69 & \\
\hline
\end{tabular}

Auf den bundesweit vorhandenen Notarztprotokollen werden verschiedene Zeiten erfasst, wodurch Intervalle wie Transportintervall, Prähospitalzeitintervall sowie Ausrückintervall errechnet werden können. Das "On-Scene-Intervall", die notärztliche Versorgungsphase betrug in einer Studie von BURGHOFER et al. (2006) im Mittel 21 Minuten, sie beinhaltete die Patientenversorgung sowie den Zugangs- und Abtransport zum Rettungsmittel. Die Zeitdauer war hierbei von einer eventuellen Vorbehandlung und der Verletzungsschwere abhängig - dementsprechend bei Schwerverletzten verlängert. Retrospektiv von SCHLECHTRIEMEN et al. (2002) ausgewertete Daten zeigten, dass $35,7 \%$ der Patienten mit Polytrauma mittels 


\section{Einleitung}

Luftrettungsdienst innerhalb von einer Stunde in die Klinik eingeliefert wurden. In einer weiteren Studie wurden bei 82,3\% aller Einsätze ein Prähospitalintervall von unter einer Stunde eingehalten, was der Forderung nach Nutzung der "golden hour of trauma" (COWLEY 1977) entgegen kommt. Im bodengebundenen Rettungsdienst wurden präklinische Versorgungszeiten von 15-20 Minuten beobachtet (FINKENZELLER et al. 2005). Insbesondere polytraumatisch verletzte Patienten profitieren von einer kurzen Rettungszeit (<30min) und schnellen Einlieferung in ein Traumazentrum (<90min) (GELDNER und SCHWARZ 2003). Aufgrund größerer zu versorgender Flächen und den schlechteren Straßenverhältnissen ist mit einer verlängerten Transportzeit polytraumatisierter Patienten in das entsprechende Krankenhaus auszugehen und mit einer Verschlechterung der Gesamtprognose. Dies ist vor allem in den neuen Bundesländern der Fall (KÜHNE et al. 2006). Ein optimale Traumaversorgung ist nicht nur durch eine adäquat ausgerüstete Maximal- und Schwerpunktklinik gewährleistet, sondern es müssen auch die infrastrukturellen Bedingungen in den einzelnen Bundesländern der Situation angepasst werden. Die gezielte und zeitgerechte Zuweisung in die geeignete Klinik sowie das hohe notärztliche Versorgungsniveau gewinnen zunehmend an Bedeutung (LUIZ 2003).

Am Unfallort werden zunächst Unfallhergang, die Umgebungssituation sowie das Ausmaß des Traumas erfasst. Falls noch nicht erfolgt, muss die Einsatzstelle zum Ausschluss der Eigen- und Fremdgefährdung entsprechend gesichert werden (BECK et al. 2002, HAUKE et al. 2001, TREPESCH et al. 2001).

Es wurden verschiedene Algorithmen für das präklinische Management des Polytraumas vorgeschlagen und drei Phasen für ein zeitgerechtes standardisiertes Vorgehen entworfen (NEHRLICH und MAGHSUDI 1997, KANZ et al. 2002):

se ALPHA : - Lebensrettende Maßnahmen 1. Minute

Pha

- Kontrolle der Vitalparameter mit präklinischem Monitoring (SpO2, EKG, Blutdruck und bei beatmeten Patienten dann Kapnometrie)

- Kontrolle der Bewusstseinslage anhand des GCS

- Kontrolle und Sicherung der Atemwege

- Atmung

- Kreislaufkontrolle (durch Palpation des Karotispulses)

- ggf. Notfallintubation unter HWS-Stabilisierung

- ggf. Reanimation. 


\section{Einleitung}

se BRAVO : $\quad-5 \mathrm{~min}$

Pha

- spritzende Blutung mittels Kompression und

Druckverband versorgt

- Stabilisierung der HWS mittels Stiffneck

- Sauerstoffapplikation (10l/min)

- Volumentherapie (1000ml kristalline Lösung im Sinne einer Small-Volume-Resuscitation (SVR) über

2 großlumige periphere Venenzugänge).

Se CHARLIE : - $\quad$ 15min

- Beurteilung des Verletzungsmuster (dieses wird an Rettungsleitstelle weitergegeben, um zeitnahe Suche nach geeigneten Zielklinik zu ermöglichen)

- Analgesie, Sedierung

- Frühintubation, Beatmung (Ziel ist suffiziente Oxygenierung und Ventilation und ein sicherer Aspirationsschutz)

- Anlage einer Thoraxdrainage

- Lagerung und ggf. vorherige Reposition von Luxationen sowie groben Fehlstellungen von Frakturen (Vakuummatratze/-schienen etc.)

- Transport.

Es ist hierbei anzumerken, dass das präklinische Atemwegsmanagement von Traumapatienten oft situationsbedingt deutlich erschwert ist. Laut Untersuchung von MUTZBAUER und HELM (2001) zeigte sich in einer retrospektiven Studie bei 162 eingeklemmten Patienten, die durch den Rettungshubschrauber Christoph 22 versorgt wurden, dass 31\% noch im eingeklemmten Zustand sowie 38\% direkt nach Extraktion endotracheal intubiert werden mussten. Schwerwiegende Komplikationen nach einer erfolgreichen Intubation können durch eine korrekte Fixierung des Tubus und kontinuierliche klinische Kontrolle auch durch Kapnographie verhindert werden. Als Alternativen zur Atemwegssicherung bei Unmöglichkeit der Intubation stehen Combitubus, Larynxtubus und Larynxmaske zur Verfügung. Als Ultima Ratio gilt die Notfallkoniotomie als chirurgischer Zugang zu den Atemwegen (KEUL et al. 2004, ZINK et al. 2002).

"Eine Analgosedierung schützt vor streßinduzierten Folgeschäden und rettungstechnischen nicht vermeidbaren schmerzhaften Manipulationen und Erschütterungen." (BERNHARD et al. 2004, S.902). Damit es aufgrund des 


\section{Einleitung}

verminderten Verteilungsvolumen nicht zu einer Überdosierung und konsekutiver Ateminsuffizienz kommt, müssen die Medikamente titrierend appliziert werden (MÖNK 2003).

Die Präklinische Volumentherapie ist unter anderem vom Verletzungsmuster abhängig, so wird beim SHT eher eine aggressive Volumensubstitution mit Ziel normotensiver Blutdruckwerte empfohlen. Demgegenüber wird bei unkontrollierbaren Blutungen in Körperhöhlen eher eine permissive Hypotension angestrebt (KREIMEIER et al. 2003).

Die rasche präklinische Versorgung und der entsprechend zeitnahe Transport in die geeignete Klinik führen zu einer erhöhten Überlebensrate nach schwerem Trauma. Die Auswahl des geeigneten Transportmittels muss jeweils individuell unter Berücksichtigung des Verletzungsmusters, der Entfernung zur Zielklinik, der lokalen Wetterbedingungen und der Tageszeit erfolgen (BIEWENER et al. 2004). Von der Rettungsleitstelle wird die Zielklinik über den Patienten, die Notarztdiagnosen und ob der Patient beatmet ist oder nicht, sowie über die ungefähre Eintreffzeit informiert (BECK et al. 2002, BIEWENER et al. 2004).

\subsection{Klinische Versorgung des Polytraumas}

Durch die Deutsche Gesellschaft für Unfallchirurgie wurden bereits 1997 erstmalig Empfehlungen zur Struktur, Organisation und Ausstattung der präklinischen und klinischen Verletzten-Versorgung in der Bundesrepublik Deutschland publiziert (HAAS 1997). Durch Veränderungen der Verletzungsarten und -schwere sowie der Rahmenbedingungen ist eine Überarbeitung der Empfehlungen notwendig geworden. Die Arbeitsgemeinschaft Polytrauma der Deutschen Gesellschaft für Unfallchirurgie gründete 1993 das Traumaregister, es dient als Instrument der multizentrischen Erfassung von Behandlungsdaten Schwerverletzter im deutschsprachigen Raum. Seit April 2003 erfolgt die Erfassung der Daten online. Ziel dieses Zusammenschlusses ist die Verbesserung der Versorgung Schwerverletzter (www.traumaregister.de 2000). 
Zur Versorgung der Schwerverletzten wurden die in Deutschland vorhandenen stationären Versorgungseinrichtungen in 3 Gruppen gegliedert:

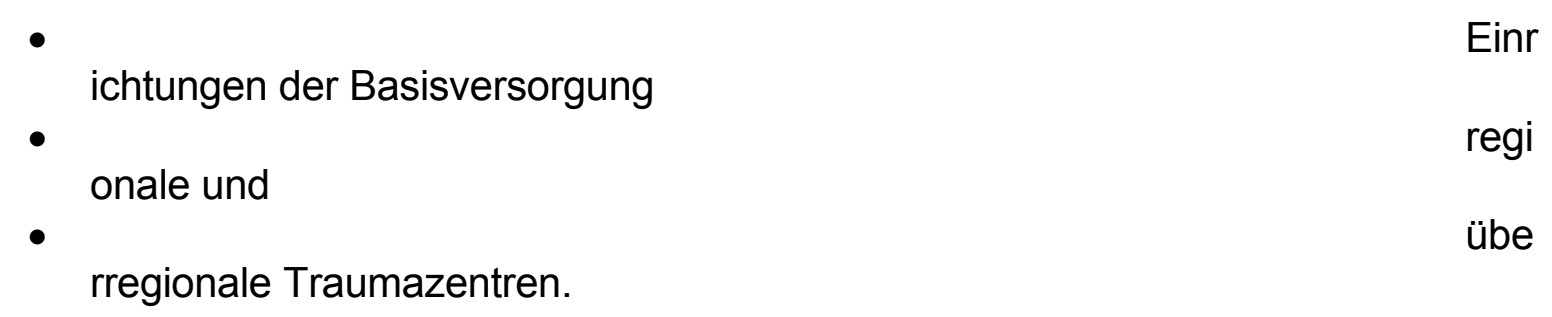

Einr regi übe

Wichtige Voraussetzung für diese Gliederung ist auch eine strukturierte Verzahnung, ein entsprechender Informationsaustausch und enge Kooperation zwischen den jeweiligen Kliniken (Deutsche Gesellschaft für Unfallchirurgie e.V. September 2006).

Laut KÜHNE et al. (2006) wurden 108 Häuser mit ca. 8700 unfallchirurgischen Betten als überregionale Traumazentren, 209 Kliniken mit ca. 12000 unfallchirurgischen Betten als regionale Traumazentren und 431 Krankenhäuser mit ca. 37000 chirurgischen Betten der Basisversorgung zugeordnet (Stand 31.12.2004).

Aufgabenstellung und Strukturelle Anforderungen der Versorgungsstufen nach dem "Weißbuch" Schwerverletzten-Versorgung (Deutsche Gesellschaft für Unfallchirurgie e.V. September 2006, S.21-22):

\section{A) Einrichtungen der Basisversorgung:}

- Sic herstellung der Behandlung Schwerverletzter durch chirurgische Notfallversorgung (z.B. Notfallthorakotomie, Notlaparotomie, Stabilisierungen mittels Fixateur oder Beckenzwinge)

- $\quad$ Ver pflichtung zur Mit- und Weiterbehandlung im Verbund mit den anderen Traumazentren entsprechend ihrem Leistungsspektrum

onale Teilnahme an klinischen Studien opti - $\quad$ Bet eiligung an fachspezifischen Fort- Weiterbildungen

alitätssicherung

eilung für Unfallchirurgie od. Unfallchirurgie/Orthopädie od. Chirurgie stündige Verfügbarkeit FA für Orthopädie/Unfallchirurgie Zusatzweiterbildung Spezielle Unfallchirurgie

Qu

Abt 24mit 


\section{Einleitung}

\footnotetext{
stündige Verfügbarkeit FA für Viszeralchirurgie od. Allgemeinchirurgie stündige Verfügbarkeit FA für Anästhesiologie

stündige Bereitschaft der Notaufnahme

stündige Operationsbereitschaft für Notfälle mliche, apparative und personelle Ausstattung zur Triage und Versorgung Schwerverletzter.
}

-

\section{B) Regionales Traumazentrum:}

stündige Aufnahme und Versorgung von Schwerverletzten haltung bestimmter Fachdisziplinen nfalls Verpflichtung zur Mit- und Weiterbehandlung gemäß Leistungsspektrum

\section{$\bullet$} etzungsartenverfahren der gesetzlichen Unfallversicherungen

nahme an klinischen Studien

allchirurgische Qualitätssicherungsverfahren

unf

ik für Unfallchirurgie od. Unfallchirurgie/Orthopädie

- 24 stündige Präsenz FA für Orthopädie/Unfallchirurgie mit Zusatzweiter-bildung Spezielle Unfallchirurgie

stündige Präsenz FA für Viszeralchirurgie od. Allgemeinchirurgie

stündige Präsenz FA für Anästhesiologie

stündige Präsenz FA für Radiologie ndige Verfügbarkeit wesentlicher verletzungsrelevanten Fach-abteilungen

stündige Bereitschaft der Notaufnahme

stündige Operationsbereitschaft zur Versorgung mind. eines Schwerverletzten

stündige Vorhaltung entsprechender Intensivbehandlungskapazität 


\section{Einleitung}

eiligung an der präklinischen Notfallrettung (RTH/Notarztstandort)

Bet

-

mliche, apparative und personelle Ausstattung entsprechend dem Verletzungsartenverfahren.

\section{C) Überregionales Traumazentrum:}

• Versorgung der Schwerverletzten => insbesondere interdisziplinäre Behandlung aller Patienten

und Weiterbehandlung (Sekundärverlegung) von Schwerverletzten

etzungsartenverfahren der gesetzlichen Unfallversicherungen

Mit-

Verl währleistung der gesamten fachspezifische Aus-, Fort- und Weiterbildung

$\mathrm{Ge}$

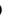
nahme an wissenschaftlichen und klinischen Studien

Teil

unf

allchirurgische Qualitätssicherungsverfahren

Klin

ik für Unfallchirurgie od. Unfallchirurgie/Orthopädie

stündige Präsenz FA für Orthopädie/Unfallchirurgie mit Zusatzweiter-bildung Spezielle Unfallchirurgie

-

stündige Präsenz FA für Viszeralchirurgie od. Allgemeinchirurgie

stündige Präsenz FA für Anästhesiologie

stündige Präsenz FA für Radiologie

-

stündige Präsenz FA für Neurochirurgie

ndige Verfügbarkeit aller verletzungsrelevanten Fachabteilungen

stündige Bereitschaft der Notaufnahme

stündige Operationsbereitschaft zur Versorgung von mind. zwei Schwerverletzten mit entsprechendem Personal

stündige Vorhaltung entsprechender Intensivbehandlungskapazität für mehrere gleichzeitig zu behandelnden Schwerverletzten

ung oder maßgeblich Beteiligung an der präklinischen Notfallrettung (RTH/Notarztstandort)

-

mliche, apparative und personelle Ausstattung entsprechend dem Verletzungsartenverfahren. 


\section{D) Spezialisierte Behandlungszentren:} werbrandverletzte

kenmarksverletzte

Rüc

plantationszentrum $\operatorname{Re}$ $\operatorname{Re}$ habilitation von Schädel-Hirn-Verletzter.

Die apparative Ausstattung der Zentren ist im Anhang aufgeführt. (siehe "Anforderungen an Krankenhäuser zur Behandlung von Schwerverletzten im Rahmen des Traumanetzwerkes" aus Weißbuch Schwerverletzten-Versorgung (Deutsche Gesellschaft für Unfallchirurgie e.V. September 2006).

Die Aufnahme der Patienten erfolgt über den sogenannten Schockraum der Klinik. Die Behandlung dort sollte auf klinikinternen, interdisziplinären Behandlungsrichtlinien in Anlehnung an die S3-Leitlinie der Deutschen Gesellschaft für Unfallchirurgie (DRESING et al. 2007). Die erstversorgenden anwesenden Chirurgen und Anästhesisten sollten in ihrer Ausbildung einen Advanced Trauma Life Support (ATLS)-Kurs erfolgreich absolviert haben (BOUILLON et al. 2004, STURM et al. 2002).

Der Transport in den Schockraum eines regionalen oder überregionalen Traumazentrums ist bei Verdacht auf eine schwerwiegende Verletzung bzw. hohen Gefährdungsgrad des Patienten angezeigt. Hierüber entscheidet der Notarzt unter Zuhilfenahme folgender Kriterien aus dem "Weißbuch" Schwerverletzten Versorgung (Deutsche Gesellschaft für Unfallchirurgie e.V. September 2006, S.20):

1.

Iwerte

Vita

sgow-Coma-Scale $<14$

Gla

$$
\text { - }
$$

olischer Blutdruck unter $90 \mathrm{mmHg}$

Syst

mfrequenz unter 10 oder über 29/min Ate

erstoffsättigung unter $90 \%$ 


\section{Einleitung}

2.

etzungsmuster

Verl

weres SHT

Sch

ennbar schwere Abdominalverletzung

Erk

abiler Thorax

Inst

ne Thoraxvertet

Offe

ne Thoraxverletzung

Inst

abile Beckenfraktur

-

r als 1 Fraktur großer Röhrenknochen der unteren Extremitäten

Meh

Sta

mmnahe Gefäßverletzung

Pro

ximale Amputationen

3.

allmechanismus

Unf

Fuß

gänger oder Fahrradfahrer angefahren $(>30 \mathrm{~km} / \mathrm{h})$

Mot

orrad- oder Autounfall mit hoher Geschwindigkeit

Her

ausschleudern aus dem Fahrzeug

-

osserieverformung über $50 \mathrm{~cm}$

Kar

Tod

eines Beifahrers

Stur

z aus mehr als 3 Meter Höhe

Expl

osionsverletzung

Eink

lemmung/Verschüttung.

Bei Transportzeiten von mehr als 30 Minuten sollte ein nächstliegendes Krankenhaus der Basisversorgung angefahren werden, um den Patienten entsprechend der ATLSKriterien zu versorgen und die Transportfähigkeit für eine Weiterverlegung herzustellen. Eine zeitgerechte Verlegung sollte hierbei angestrebt werden.

Schon während der polytraumatisierte Patient zum Krankenhaus transportiert wird, wird das Schockraumteam benachrichtigt, damit es bei Eintreffen des Patienten bereitsteht. Desweiteren wird es über den Zustand sowie das Verletzungsmuster des Patienten informiert. Trifft nun der Patient in Notarztbegleitung im Schockraum ein, so übergibt dieser den Patienten an den sog. Traumaleader, indem er alle inm bekannten 
Informationen über Unfallhergang, Zustand des Patienten und Therapiemaßnahmen am Unfallort kurz berichtet. Nun beginnt das klinische Management, hierbei laufen Diagnostik und Therapie parallel, wobei die Diagnostik bei Notwendigkeit notfalltherapeutischer Interventionen jederzeit abgebrochen wird.

Laut der Leitlinie "Polytrauma" der DGU, unterscheidet man folgende Behandlungsphasen (DRESING et al. 2007, S.14) :

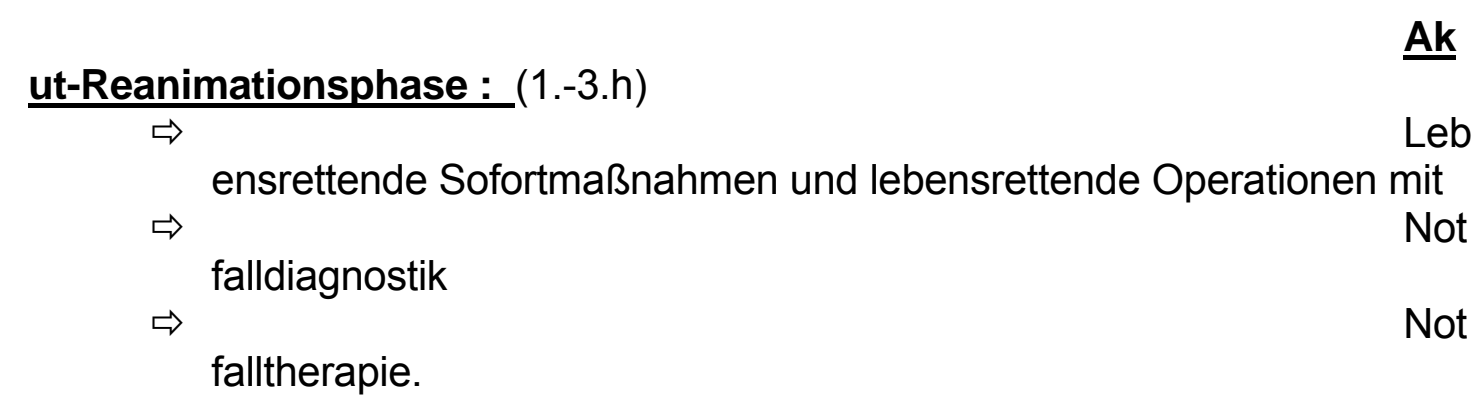

undärphase : (3.-8.Tag, Regenerationsphase)

$\Rightarrow$

nsivtherapie

$\Rightarrow$

tere Diagnostik

$\Rightarrow$ ngliche Operationen.
Inte

Wei

Dri

$\underline{\text { Ter }}$

Auf

$\operatorname{Re}$

tiärphase : (>8.Tag, Rehabilitationsphase)

$\Rightarrow$

geschobene Operationen

$\Rightarrow$

habilitationsmaßnahmen.

Die Verletzungsmuster bzw. die Verletzungsschwere müssen im Schockraum bereits klar definiert werden. An Hand eines kurzen klinischen Untersuchungsganges sowie ergänzend durch Röntgenuntersuchung und zunehmend auch durch GanzkörperComputertomographie wird das Verletzungsmuster dargestellt (WOLTMANN und BÜHREN (2007). Nachfolgend ist ein kurzer klinischer Untersuchungsgang aufgeführt: 
- Untersuchung des Kopfes :

$\Rightarrow \quad$ äuß

ere Verletzungszeichen, Hämatome, Blutungen, Offene Verletzungen

$\Rightarrow$ illen (Seitendifferenz, Pupillenverengung/-erweiterung, Lichtreaktion)

$\Rightarrow$ ungen aus Nasen-Rachen-Raum oder Gehörgang

$\Rightarrow$ uoraustritt aus Nase oder Ohr

$\Rightarrow$ ningismus

Blut Liq $\mathrm{Me}$

$\Rightarrow$ Fra $\Rightarrow$ kturzeichen und Instabilität Verl etzung der Halswirbelsäule.

- Untersuchung des Thorax :

$\Rightarrow$ Inspektion (Wunden, Prellmarken, Hautemphysem, Atemexkursion)

$\Rightarrow$ Perkussion

$\Rightarrow$ Auskultation (Pneumothorax, Spannungspneumothorax, etc.)

$\Rightarrow$ Kompression des knöchernen Gerüstes einschließlich Rippen, Sternum und Claviculae.

- Untersuchung des Abdomens :

$\Rightarrow$ Inspektion (Wunden, Prellmarken, penetrierende Verletzungen)

$\Rightarrow$ Auskultationsbefund und Palpationsbefund sind beim Polytrauma meist wenig hilfreich, da der Patient häufig keine Schmerzen äußern kann, da er oftmals bewusstlos oder intubiert ist.

$\Rightarrow$ Bei stumpfen Bauchtraumen weitere Diagnostik durch Sonographie möglich.

- Untersuchung der Wirbelsäule :

$\Rightarrow$ Inspektion (Wunden, Prellmarken, Hämatome)

$\Rightarrow$ Fehlstellung, abnorme Beweglichkeit

$\Rightarrow$ Schmerzlokalisation

$\Rightarrow$ Neurologische Ausfälle (Querschnittsymptomatik).

- Untersuchung des Beckens und der Extremitäten :

$\Rightarrow$ Inspektion (Wunden, Prellmarken, Fehlstellungen, Austritt von Blut aus der Harnröhre erfordert eine retrograde Urethrographie)

$\Rightarrow$ rektale und vaginale digitale Untersuchung sind obligat

$\Rightarrow$ das Becken wird in lateraler und anteroposteriorer Richtung vorsichtig komprimiert, Schmerzen oder abnorme Beweglichkeit weisen auf eine Verletzung hin - CAVE : Blutverlust

$\Rightarrow$ Extremitäten (Blutung, Hämatom, Kontrolle der Stabilität, abnorme Beweglichkeit, Krepitation, Fehlstellung - Hinweis auf eine Fraktur)

$\Rightarrow$ Palpation der Pulse - Hinweis auf Gefäßverletzung.

(STÜRMER et al. 2001, DRESING et al. 2007).

Diese Untersuchung kann ein erfahrener Arzt innerhalb weniger Minuten durchführen und erhält ohne großen Aufwand einen Überblick über das Verletzungsmuster des Patienten. Des weiteren können evtl. fremdanamnestisch Daten zu den Vorerkrankungen (Herzschrittmacher, Diabetes, usw.) und zu etwaigen Begleit- 


\section{Einleitung}

umständen (Gerinnungsmedikation, Schwangerschaft, etc.) erfragt werden. Neben der klinischen Untersuchung laufen parallel die laborchemischen Analysen von Blut und Urin. Folgende Laborwerte sollten notwendigerweise bestimmt werden :

$\begin{array}{lr}\text { - } \quad \text { bild } & \text { Blut } \\ \text { - } \quad \text { gruppe } & \text { Blut } \\ \text { - } \quad \text { innung } & \text { Ger } \\ \text { - } \quad \text { ktrolyte } & \text { Ele } \\ \text { - uzblut } & \text { Kre } \\ \text { gasanalyse. } & \text { Blut }\end{array}$

Fakultativ können Laktat, die Hepatitis- und HIV-Serologie (zum Schutz der Mitarbeiter), Blutalkoholspiegel, Drogenscreening und Medikamentenscreening, sowie das Myoglobin bestimmt werden (STÜRMER et al. 2001).

Nach Abschluss der klinischen Untersuchung kann die apparative Diagnostik starten. Dazu zählen die schon erwähnte Sonographie des Abdomens und des Thorax, die auch später zu Kontrollen wiederholt werden. Bei der Röntgenkontrolle erfolgt unverzüglich die Anfertigung von Thorax ap, Becken ap und HWS seitlich. Die Computertomographie wird bei progredienten Hirndruckzeichen und fehlenden Hinweisen auf lebensbedrohliche Blutungen im Thorax und Abdomen frühzeitig eingesetzt. In einer Studie von WOLTMANN und BÜHREN (2007) zeigte sich durch Einsatz des Polytrauma-CTs eine Verkürzung der Schockraumphase in einem Zeitraum von 2,5 Jahren von durchschnittlich 144 Minuten auf 88 Minuten, sowie Senkung der Mortalität von $23 \%$ auf $12 \%$.

In Deutschland wurde seit 2003 das Konzept des "Advanced Trauma Life Support" (ATLS) zunehmend angewandt bzw. standardisierte Algorithmen für das Schockraummanagement erstellt, um Behandlungsfehler zur reduzieren und eine Verkürzung und Optimierung der Behandlungsabläufe zu schaffen (BOUILLON et al. 2004). Parallel zur klinischen Erstuntersuchung erfolgt bei entsprechenden Befunden die frühe klinische Therapie durch Reanimationsmaßnahmen, Überprüfung der 


\section{Einleitung}

Tubuslage, Legen von venösen und arteriellen Zugängen, Infusionstherapie (bei anhaltendem schweren Schock auch Blutsubstitution, im Notfall auch Blutgruppe 0 rh negativ), Kompressionsverbände bei Blutungen. Erste Notfalloperationen sind unter anderem Notlaparotomie, Notthorakotomie, Thoraxdrainage, Operative Versorgung stammnaher Massenblutungen sowie ggf. bei Beckenzerreißung Anlage einer Beckenzwinge. Wichtige medikamentöse Gabe besteht in kreislaufstabilisierenden Medikamenten, Tetanusprophylaxe sowie Antibiotikaprophylaxe (DRESING et al. 2007).

Die apparative Diagnostik wird bei ausreichender Stabilität des Patienten durch Kontrollsonographie des Abdomens und Thorax sowie konventionelles Röntgen von :

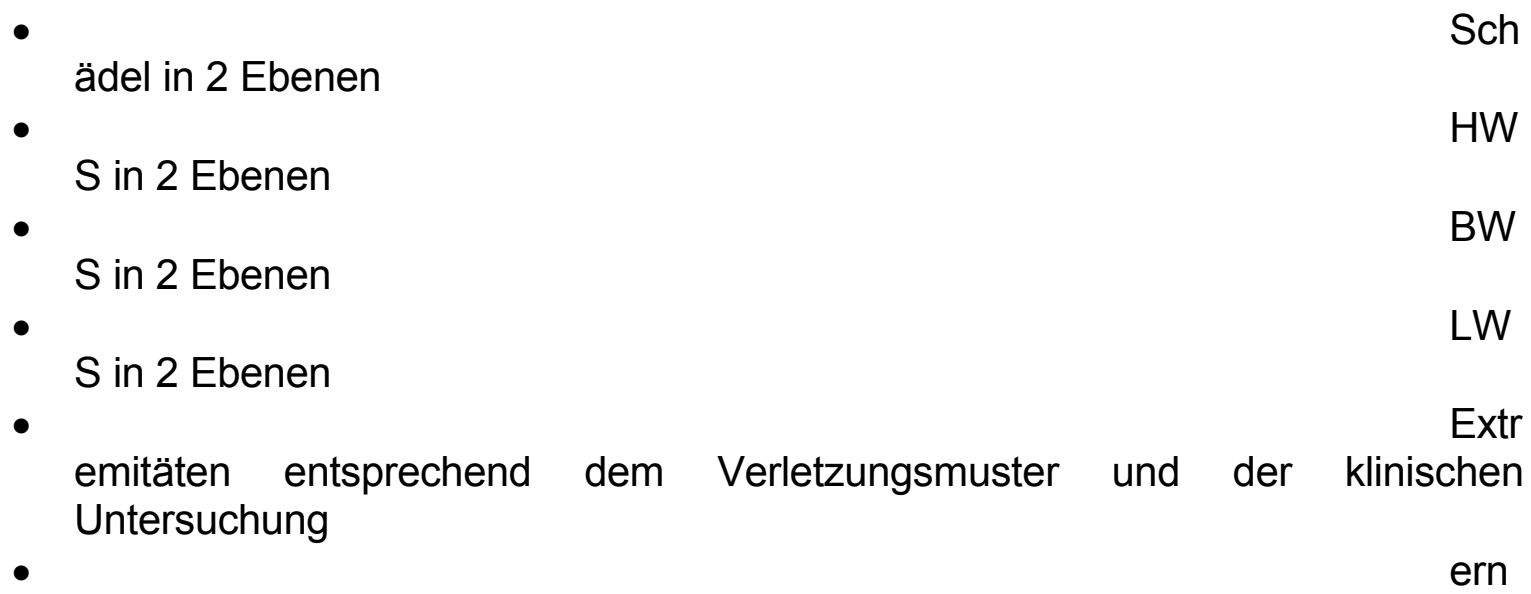
eute Thoraxkontrolle

vervollständigt (DRESING et al. 2007,S.21).

Weitere ergänzende apparative Diagnostik bietet die Doppler-Sonographie, Angiographie und Angio-CT, Urethrographie (STÜRMER et al. 2001).

Aufgrund eines zunehmenden Verständnisses der pathophysiologischen Vorgänge bei Trauma und Operation erfolgte in den letzten Jahren eine Anpassung der Versorgungskonzepte bzw. -zeitpunkte bei Schwerverletzten. Es werden hierbei zwei Konzepte unterschieden: (HAAS et al. 2007)

$\Rightarrow$ Da mage-control (DC)-Konzept 


\section{Einleitung}

$\Rightarrow$

y-total-care (ETC)-Konzept.

Das Damage-control (DC)-Konzept sieht entsprechend dem Verletzungsmuster des Patienten ein abgestuftes Versorgungsschema vor. Dem zugrunde liegend erkannte man, dass zu lange primäre Eingriffe dem schwerverletzten Patienten durch Verstärkung einer letalen Trias (metabolische Azidose, Hypothermie, Koagulopathie) eher schaden und zum sog. "second hit" führen (PAPE et al. 1999 und 2002, KEEL und TRENTZ 2005).

Das ursprüngliche DC-Konzept wurde in 3 Phasen unterteilt : 1. Phase der Erstoperation, 2. der Stabilisierung und 3. der geplanten Reoperation; eine weitere Phase, die "ground zero recognition phase" = präklinische/frühe klinische Phase wurde ergänzt (HAAS et al. 2007). Die erste Phase des DC-Konzeptes beinhaltet Operationen, durch die lebensbedrohliche Blutungen kontrolliert bzw. Körperhöhlen entlastet und einer Kontamination vorgebeugt werden können. Auch für die unfallchirurgische Versorgung wurde dieses Konzept übernommen, hierbei geht es um eine schnelle Stabilisierung der vorhandenen Frakturen mittels Fixateur extern und eine entsprechende Weichteilversorgung (PAPE und KRETTEK 2003, GIANNOUDIS et al. 2009, TUTTLE et al. 2009). In einer Prospektiven Studie von TAEGER et al. (2005) konnte durch Anwendung des DC-Konzeptes eine signifikante Verkürzung der OP-Zeit in der Primärphase und ein geringerer Blutverlust verzeichnet werden. In der sich anschließenden Stabilisierungsphase wird der Patient intensivmedizinisch stabilisiert, dass heißt Ausgleich der Azidose und Temperatur, gute Oxygenierung, Gerinnungsnormalisierung (PARR und ALABDI 2004). Hiernach kann der Patient bei ausreichender Stabilisierung den geplanten Reoperationen zugeführt werden.

Das Early-total-care-Konzept steht hierzu im Gegensatz und wird seit den 1980er Jahren propagiert. Es beinhaltet die definitive Versorgung von Extremitätenverletzungen in der Primärphase, wodurch im klinischen Verlauf eine frühe Mobilisation und Schmerzreduktion erreicht würde (BONE et al.1989, JOHNSON et al. 1985). Es gibt jedoch dazu sehr unterschiedliche Studien, die belegen, dass vor allem bei einem zusätzlichen SHT oder schwerem Thoraxtrauma es durch die Länge der primären Operation zum Beispiel durch intramedulläre Nagelung zu einem zusätzlichen Schaden kommt (PAPE et al. 2004). In einer Studie von PAPE et al. 
(2002) wurde gezeigt, dass bei der Anwendung des ETC-Konzeptes signifikant höhere Raten an ARDS ("acute respiratory distress syndrom") und MOV (Multiorganversagen) kommt.

Für die Frakturversorgung werden die beiden Konzepte immer noch kontrovers zwischen den Verfechtern des ETC-Konzeptes (Nordamerika) (O'BRIEN 2003) und DCO-Anhängern diskutiert. In Deutschland hat sich zunehmend ein abgestuftes Versorgungskonzept bei der Frakturversorgung des Polytraumatisierten etabliert (NAST-KOLB et al. 2005, PAPE et al. 2002, PAPE et al. 2004). Hierbei fanden bestimmte Patientencharakteristika ("Borderline-Patienten"), welche Komplikationen erwarten lassen, Beachtung und es wurde sich zu einem abgestuften Vorgehen entschieden (PAPE und KRETTEK 2003).

Sogenannte "Borderline-Kriterien" für ein abgestuftes Versorgungskonzept sind (PAPE et al. 2004, S.933):

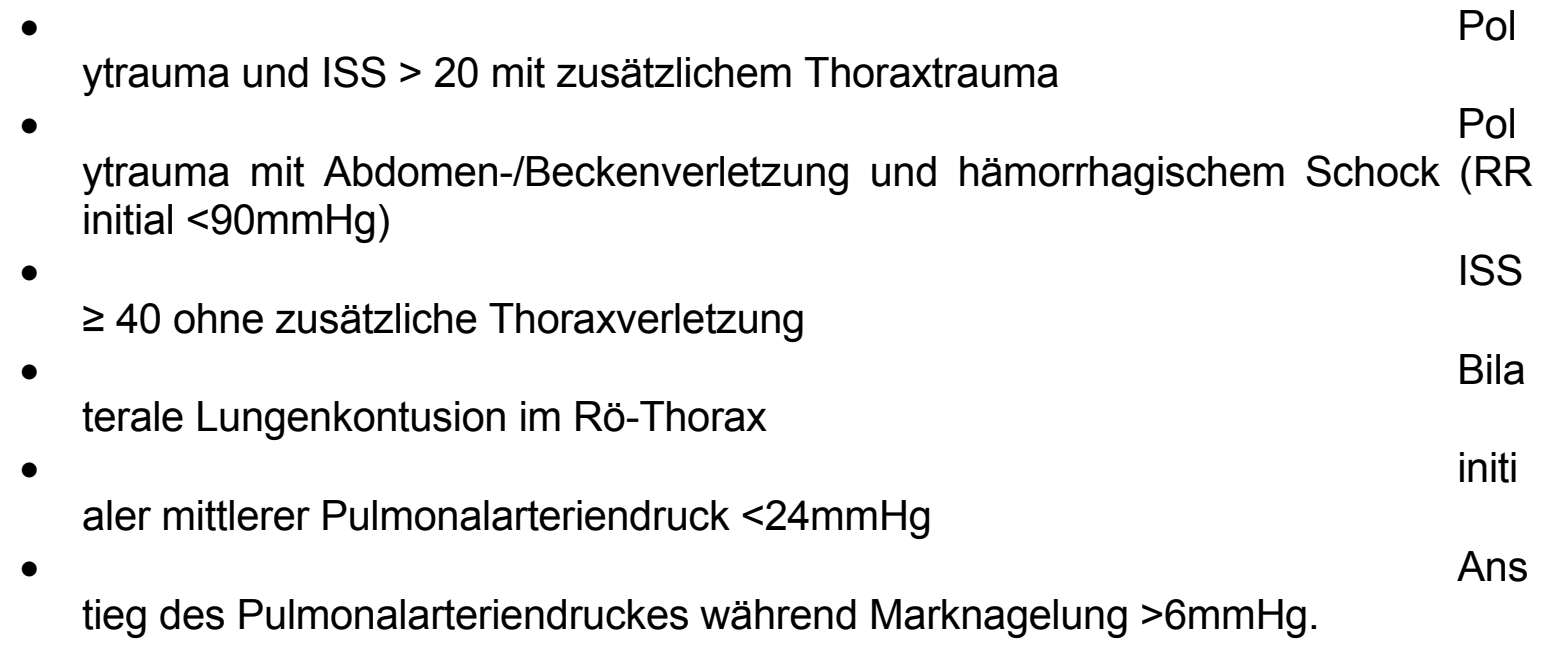

Für eine erfolgreiche Anwendung des DC-Konzeptes ist es wichtig, den richtigen Zeitpunkt für die sekundäre definitive Versorgung der Fraktur zu wählen. Es zeigte sich in mehreren Studien, dass der beste Zeitpunkt für die verzögerte Versorgung zwischen dem 5. und 10.Tag nach Trauma liegt, während der Zeitraum zwischen dem 2.-4.Tag durch eine hyperinflammatorische Phase und der Zeitraum zwischen der 2.-3.Woche 


\section{Einleitung}

aufgrund der Immunsuppression gemieden werden sollte (PAPE et al. 1999, PAPE et al. 2002).

Fazit : "Die Akut- und Primärphase sind die entscheidenden Phasen für die Festlegung und Durchführung einer prioritätenorientierten (operativen) Behandlungsstrategie unter Berücksichtigung des entstandenen Verletzungsmusters sowie des Zustandes des Patienten." (HAAS et al. 2007,S.900)

Ist die stationäre Behandlung erfolgreich abgeschlossen, schließt sich in den meisten Fällen eine Rehabilitationsmaßnahme an.

\subsection{Rehabilitation nach Polytrauma}

Das Ziel der Rehabilitation mittels medizinischer, beruflicher und sozialer Maßnahmen ist die Wiedereingliederung des polytraumatisierten Patienten in sein soziales und berufliches Umfeld. Die Rehabilitation steht vor einer Entschädigung durch geldliche Mittel. Die Träger für eine Rehabilitation sind unter anderem die Gesetzlichen Krankenversicherungen, die Gesetzlichen Unfallversicherungen, die Gesetzlichen Rentenversicherungen, die Altershilfe für Landwirte und die Kriegsopferversorgung.

Man unterscheidet 3 Formen der Rehabilitation: die medizinische, die berufliche und die soziale Rehabilitation.

Die medizinische Rehabilitation beginnt meist schon während des stationären Aufenthaltes des Patienten und sollte auch gleich im Anschluss daran weitergeführt werden. Hierfür stehen 3 Zentren zur Verfügung :

1.)Rehabilitationszentrum für Hirngeschädigte

2.)Rehabilitationszentrum für Verletzungen des Bewegungsapparates

3.)Rehabilitationszentrum für Querschnittverletzte.

Schon kurz nach Aufnahme des Patienten nach dem Trauma beginnt die Physiotherapie. Sie dient in den ersten Tagen als Prophylaxe für vielleicht entstehende Komplikationen, wie zum Beispiel Dekubitus, Pneumonie, Thrombosen und 


\section{Einleitung}

Kontrakturen. Dazu dienen unter anderem die Atemgymnastik, die aktive und passive Bewegung der nicht verletzten Extremitäten, sowie die richtige Lagerung der verletzten Extremitäten auf Schienen (WOLTER et al. 1997, SIMMEL und BÜHREN 2009).

Möglichst frühzeitig sollte hiernach der Patient aus dem Bett im Gehwagen oder an Unterarmgehstützen mobilisiert werden. Ziel der Physiotherapie bei Verletzungen der Wirbelsäule und der Extremitäten ist eine Erhaltung bzw. das Wiedererreichen der Gelenkfunktion sowie der Muskelkraft und der Koordination der Bewegungsabläufe. Gelingt das nicht, müssen „kompensatorische Bewegungsmuster“ erlernt werden. Um dies zu unterstützen führt man passive Dehnungstechniken, Entspannungstechniken und Traktions-Behandlungen durch. Auch technische Geräte können dabei Anwendung finden, wie zum Beispiel die passive Durch-Bewegung auf der Motorschiene. Wenn nach diesen Behandlungen eine ausreichende Belastbarkeit erreicht wurde, kann mit der aktiven Therapie begonnen werden. Hierunter zählt nicht nur das Bewegungsbad, sondern auch isometrische Anspannungsübungen oder auch die Trainingstherapie an Fitnessgeräten. Bei Verletzungen der unteren Extremität ist man bestrebt, den Patienten möglichst früh wieder auf die Beine zu stellen und inm das Gehen wieder zu erlernen. Hierfür muss der Patient im Umgang mit bestimmten Hilfsmitteln wie Bandagen oder Gehhilfen geschult werden. Das Gehen kann auf der Ebene, der Treppe oder dem Laufband unter Zuhilfenahme der dafür notwendigen Hilfsmittel trainiert werden. Bei narbigen Verhärtungen oder Verklebungen der Gleitschichten, sowie Lymphödem oder ähnlichem kommt die physikalische Therapie, wie zum Beispiel die Lymphdrainage, Massage, Reizstrombehandlung oder Fangopackung zum Einsatz (WOLTER et al. 1997).

In einer Studie von STALP et al. (2001) wurde ein standardisiertes Instrument zur quantitativen und reproduzierbaren Rehabilitationserfassung nach Polytrauma ("HASPOC") entwickelt.

Parallel zur medizinischen Rehabilitation setzt auch die berufliche ein, indem dem Patienten ein Berufshelfer zur Seite gestellt wird und ihm der Einstieg zurück ins Berufsleben durch Arbeitsversuche (ggf. stundenweise Wiedereingliederung) erleichtert wird (DRESING et al. 2007). Nach verschiedenen Studien haben die Patienten mit Schädel-Hirn-Trauma die größten Schwierigkeiten wieder in den 


\section{Einleitung}

Arbeitsprozess eingegliedert zu werden (GLINZ und AFFENTRANGER 1975, ZANGGER 1989).

Auch die soziale Rehabilitation sollte schon während des stationären Aufenthaltes beginnen. Besondere Bedeutung kommt dabei der Reintegration des Patienten in sein familiäres Umfeld zu. Schon auf Station können klärende Gespräche geführt werden, inwieweit eine Rehabilitation möglich ist und mit welchen möglichen Behinderungen man auch weiterhin leben muss.

\subsection{Pathophysiologie des Polytraumas}

Pathophysiologisch betrachtet, durchläuft der schwerverletzte Patient drei Phasen. "In der ersten Phase (akute Reaktion) führt das Trauma und der hämorrhagische Schock über metabolische, neuroendokrine, inflammatorische und durch Hypovolämie getriggerte Reaktionen zum Zustand der Organe im Schock." (WOLTMANN und BÜHREN 2007, S.499). Die zweite Phase wird auch als SIRS (Systemic Inflammatory Response Syndrom) bezeichnet, hierbei führen Katabolie, Akutphasereaktion und Reperfusionsschaden zum frühen Organversagen. Es kommt zu einem autoaggressiven Circulus vitiosus mit Immundefektsyndrom und progressive Koagulopathie (MOORE et al. 1998). Laut WOLTMANN et al. (1998) kommt es durch einen "Mucosabarriere-Verlust" und "nachfolgender Translokation" bei den schwerverletzten Patienten zu einem septischen Multiorgandysfunktionssyndrom und später versterben die Patienten am Multiorganversagen mit septischen Schock. In einer Studie von WOLTMANN und BÜHREN (2007) zeigte sich, dass posttraumatisch die verzögerte zellvermittelte Immunität gestört ist, man bezeichnet dies als anerg. Es zeigte sich ferner, dass ab dem 4.Tag die später Überlebenden eine normergen Zustand erreichen, wohingegen die, die an Sepsis versterben, an- oder hyperg sind. Daraus leitet sich die Empfehlung ab, die verzögerte definitive Frakturversorgung nach dem DC-Konzept zwischen dem 5.-10.Tag nach Trauma durchzuführen. Unnötige Operationen oder Eingriffe zum falschen Zeitpunkt führen zum sog. "second-hit", hierbei wird der Circulus vitiosus autoaggressivus durch den Eingriff selbst aber auch durch Hypothermie und Hypotension während des Eingriffes verstärkt (WOLTMANN und BÜHREN 2007). 


\section{Einleitung}

Der hämorrhagisch-traumatische Schock, auch hypovolämischer Schock genannt, führt über eine Umverteilung der Makrozirkulation - durch Stimulierung des Sympathiko-adrenalen Systems und Reaktion des Renin-Angiotensin-AldosteronMechanismus zu einer Störung der Mikrozirkulation mit mikrovaskulärer Permeabilitätsschädigung und Gewebshypoxie. Die Auswirkungen auf die einzelnen Organe sind je nach Gefäßarchitektur der Endstrombahn sehr unterschiedlich (FRIEDL et al. 1989). Das Schock-Geschehen wird durch Schmerzen, die das adrenale System stimulieren, oft verstärkt oder sogar erst ausgelöst. Deshalb ist eine Ruhigstellung von Frakturen unabdingbar (OESTERN et al. 1984).

Durch vermehrtes Gewebethromboplastin und Kinasen, die aus dem traumatisch geschädigtem Gewebe stammen, kommt es zu einer vermehrten intravasalen Aktivierung der Gerinnung und zu einem verzögertem Abbau ihrer Produkte. Dies führt zur sogenannten „Hyperkoagulabilität" und damit zur disseminierten intravasalen Gerinnung (DIC). Dadurch wird die Viskosität des Blutes verstärkt und die ohnehin schon bestehende Mikrozirkulationsstörung wird noch verstärkt. Übersteigt nun der Verbrauch der Gerinnungsfaktoren deren Neubildung, kommt es zur Verbrauchskoagulopathie, deren Folge dann die hämorrhagische Diathese und Präzipitation von Fibrin mit sekundärem Organschaden sind. Wird dieser Vorgang beim Patienten nicht erkannt bzw. behandelt, kann dies in einen irreversiblen Schock münden (MÜLLERBERGHAUS et al. 1980, RIEDE und SCHAEFER 1999).

Labortechnisch lässt sich diese Gerinnungsstörung durch erhebliche Konzentrationserhöhungen an Fibrinspaltprodukten und D-Dimeren nachweisen (DÖRNER 2006). Als Therapie gelten neben der Heparinisierung auch die ausreichende Substitution von Gerinnungsfaktoren und Antithrombin-III .

BONE et al. (1989) und JOHNSON et al. (1985), Verfechter des ETC-Konzeptes, zeigten in ihren Studien, dass es durch eine späte definitive Versorgung der Frakturen der langen Röhrenknochen zu einer erhöhten ARDS-, Fettembolie- und PneumonieRate gekommen sei. Andere Studien wiederum zeigten das Gegenteil, so zeigte sich bei zusätzlichem Thoraxtrauma bei primärer Femurnagelung eine erhöhte ARDS-Rate sowie Mortalität (PAPE et al. 1993). 


\subsection{Frakturen der Extremitäten im Rahmen eines Polytraumas}

Man unterscheidet zwei Typen der Frakturheilung :

$$
\begin{aligned}
& \Rightarrow \text { indirekte Frakturheilung } \\
& \Rightarrow \text { direkte Frakturheilung (SEEBACH und MARZI 2009, S.40). }
\end{aligned}
$$

Die indirekte Frakturheilung stellt die regelhafte Heilung von Frakturen bei konservativer Behandlung, Marknagelung oder Fixateur-Behandlung dar, während die direkte vor allem bei Spaltbrüchen oder der Plattenosteosynthese zu finden ist.

Sie ist durch die Bildung eines „primären Fixationskallus“ gekennzeichnet und läuft in 5 Phasen ab :

1. Verl etzungsphase (Fraktur).

\section{Ent}

zündungsphase (gekennzeichnet durch Blutstillung und Resorption des Hämatoms, sowie Bildung eines Fibringerüstes).

3.

nulationsphase (meist überlappend zur 2 Phase stimuliert durch Wachstumsfaktoren entsteht ein neues Kollagengerüst sowie ein neues Gefäßnetz. Nach einer Zeit von 4-6 Wochen hat sich dann der Fixationskallus gebildet. Diese Kallusbildung schreitet von peripher nach zentral zur Frakturzone fort. Durch Matrixproteine werden wiederum die Osteoblasten aktiviert, die den zerstörten Knochen aufbauen.).

4.

Pha

se der Kallushärtung (Nach Kontakt der Frakturenden über den Fixationskallus kommt es zu einer zunehmenden Mineralisierung der Grundsubstanz und es bildet sich der sogenannte Geflechtknochen. Nach 3-4 Monaten hält der Knochen dann den physiologischen Belastungen stand.

5 .

Pha se des Umbaus ("modeling and remodeling") Diese Phase erstreckt sich über Monate bis Jahre. Aus dem Geflechtknochen, der durch Diffusion versorgt wird, wird durch zunehmenden Umbau der Lamellenknochen mit seinem Haverschen und Volkmannschen Kanalsystem, die seiner Ernährung dienen. Zum Abschluss des Vorgangs liegt dann Lamellenknochen mit Periost und Endost vor.

Die direkte Frakturheilung kommt ohne die Bildung eines Geflechtknochen aus. Sie beginnt mit dem Vorwachsen des Haverschen Systems in das gegenüberliegende Frakturende. Entlang der Kapillaren bilden sich dann neue Knochenlamellen. Sie wird 


\section{Einleitung}

auch als Kontaktheilung bezeichnet (SEEBACH und MARZI 2009). Voraussetzung für die Knochenbruchheilung sind eine ausreichende Durchblutung der Frakturzone, der Kontakt der Frakturenden, „eine gewisse Ruhe im Frakturspalt“, sowie die Beweglichkeit der umschließenden Muskulatur (MUHR 1997).

Die Indikation zur sofortigen Stabilisierung bzw. Versorgung der folgenden Frakturen ist selbstverständlich :

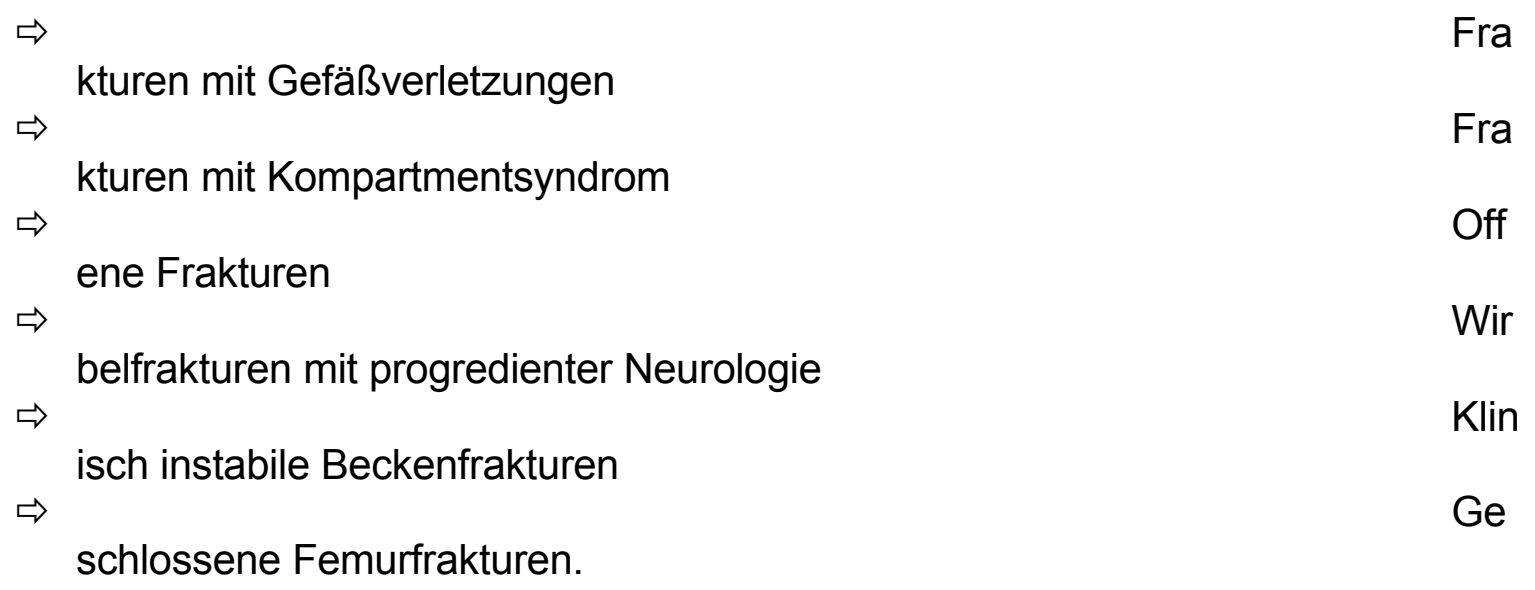

Abhängig von dem übrigen Verletzungsmuster sollte dann über den Zeitpunkt und die Art der Versorgung nachgedacht werden. Selbst bei relativ instabilem Patienten sollte zumindest eine approximative Versorgung mittels Fixateur externe versucht werden. Sobald der Patient sich stabilisiert hat, kann dann die sekundäre Versorgung der langen Röhrenknochen (vor allem Femur, Humerus und Tibia) durch die Osteosynthese erfolgen (STÜRMER et al. 2001, RIXEN et al. 2005 b, GIANNOUDIS et al. 2009).

Die Entscheidung, ob man die Extremität erhält oder ob man eine Amputation durchführen sollte, ist sehr bedeutend, denn eine primäre, zeitaufwendige Rekonstruktion kann unter Umständen zu einer lebensbedrohenden Situation führen. An der Ausdehnung und der Schwere des Weichteilschadens wie auch an der gesamten Verletzungsschwere sollte man sich bei der Entscheidung zur Amputation orientieren (TRENTZ 1993, TRUNKEY 1991).

Die rechtzeitige Amputation kann lebensrettend sein. Die Entscheidung dazu muss vom gesamten Behandlungsteam getragen werden und entsprechend dokumentiert 


\section{Einleitung}

werden, wenn nötig sogar mit Bilddokumentation der betroffenen Extremität (STÜRMER et al. 2001).

Entscheidungshilfen bieten auch die sogenannten „mangled extremity scores“, welche objektive Kriterien zur Beurteilung des Weichteilschadens, der Gesamtverletzungsschwere geben. Im angloamerikanischen Raum haben sich dafür der MESS (siehe Anhang) und der NISSSA unter Beachtung des Prinzips "life before limb" (McNAMARA et al. 1994, NAST-KOLB et al. 2005) durchgesetzt.

„Die wichtigsten Faktoren bei der Behandlung von Frakturen mit Gefäßläsion sind das Ischämieintervall und der Grad des Reperfusionsschadens." (TSCHERNE und REGEL 1997, S.218). Durch die generalisierte Hypoxämie bei einem Polytraumapatienten können Schäden, die durch diese Pathomechanismen verursacht wurden, noch verschlimmert werden. Deshalb ist es wichtig, solche Gefäßläsionen sofort zu diagnostizieren und zu behandeln. Oberste Priorität gilt deshalb der Rekonstruktion der Arterienverletzung der großen Gefäße wie A.axillaris, A.femoralis oder A.poplitea. Diese sollten primär definitiv rekonstruiert werden durch Venen oder Kunststoffinterponate. Ist das nicht möglich, so ist die Amputation indiziert (WOLTMANN und BÜHREN 2007).

Nicht zu vergessen ist die Entwicklung eines Kompartmentsyndroms, welches durch einen Anstieg des intrafaszialen Drucks zu einem irreversiblen Muskel-, Nerven- und Gefäßschaden führen kann. Wenn ein Wert gemessen wird, der die Differenz aus diastolischem Druck und $20 \mathrm{mmHg}$ überschreitet, sollte sofort eingegriffen werden und eine Fasciotomie durchgeführt werden. Deshalb sollten speziell die Hochrisikopatienten, wie zum Beispiel Patienten mit geschlossenen Trümmerfrakturen der Tibia oder auch mit komplexen Fußverletzungen, engmaschig kontrolliert werden (WHITESIDES et al. 1996).

Die offenen Frakturen müssen in der Primärperiode versorgt werden, zur Versorgung zählen das Debridement, ausgiebige Spülung und temporäre Deckung durch Hautersatzmaterialien und die stabile Fixierung (PAPE et al. 2004, LEHNERT und MARZI 2009).

In der Literatur findet man variierende Angaben über den prozentualen Anteil der Extremitätenverletzung am Verletzungsmuster, so zeigte sich in einer Datenerhebung 


\section{Einleitung}

von WENINGER et al. (2005) ein Anteil von 68,3\% mit Frakturen der langen Röhrenknochen beim Polytrauma. Am häufigsten finden sich hierbei Femurfrakturen mit einem Anteil von 73,2\%. Extremitätenverletzung in $69 \%$ der Fälle gaben auch TRUPKA et al. (1995), PIEK und JANTZEN (2000) und WALCHER (2003) bei ihren Unter-suchungen an.

\subsection{Osteosynthetische Verfahren für die Versorgung der Femurfraktur}

Entsprechend der AO-Klassifikation werden die Femurfrakturen in Femurschaftfrakturen, proximale und distale Femurfrakturen unterteilt (siehe Anhang Einteilung nach AO-Klassifikation).

Die proximalen Femurfrakturen werden unterteilt in trochantäre Femurfrakturen (31A), Schenkelhalsfrakturen (31-B) und Hüftkopffrakturen (31-C). Für die stabilen trochantären Frakturen steht die DHS (Dynamische Hüftschraube) zur Verfügung, für instabile Frakturen hingegen ist der PFN (proximaler Femurnagel) indiziert. Weitere mögliche Osteosyntheseverfahren stellen die DCS (dynamische Kondylenschraube), die Kondylenplatte sowie die DHS mit Trochanterabstützplatte dar, die in ausgewählten Fällen zur Anwendung kommen. Für die Schenkelhalsfraktur stehen zum einen kopferhaltende Verfahren und zum anderen endoprothetische Verfahren zur Verfügung. Am häufigsten wird hierbei die DHS mit zusätzlicher Rotationsschraube eingesetzt, eine weitere etwas weniger angewandte Methode ist die Osteosynthese mittels $130^{\circ}$-Winkelplatte. Bei guter Knochenqualität kann die Osteosynthese auch durch 3 Spongiosaschrauben erfolgen, welche schrittweise nachgezogen werden. Für den endoprothetischen Ersatz vor allem bei älteren Patienten findet die unipolare Kopfprothese und für aktive Patienten eher die Hüft-Totalendoprothese Anwendung. Bei den Hüftkopffrakturen sollte zuvor ein CT durchgeführt werden zur genauen Lokalisation und Größe des Fragmentes, kleine Fragmente $<1 \mathrm{~cm}^{2}$, die unterhalb Lig.capitis femoris liegen, bedürfen meist keiner Operation. Osteochondrale Fragmente, welche oberhalb liegen, sollten reponiert werden und mittels Kleinfragmentschrauben fixiert werden, die Schraubenköpfe müssen hierbei unter dem Knorpel versenkt werden (RÜEDI et al. 2003). 


\section{Einleitung}

Für die Versorgung der Femurschaftfrakturen stehen ebenfalls verschiedene Osteosyntheseverfahren zur Verfügung. Für die subtrochantären Frakturen stehen die Kondylenplatte, dynamische Kondylenschraube, proximale Femurnägel und solide Femurnägel (UFN) zur Verfügung. Die diaphysären Frakturen sind die Domäne der Marknägel. Bei einfachen Frakturen kommen die Universalnägel oder auch neuen aufgebohrten Nägel zum Einsatz. Komplizierte Frakturen werden mit dem soliden oder durchbohrten Femurnagel (UFN/CFN) versorgt. Etwas seltener kommen die LC-DCP oder die neue LCP (locking compression plate) als Brückenplatte, in tunnelierender Technik eingebracht, zur Anwendung. Bei schwerem Weichteilschaden, offenen Frakturen oder extrem polytraumatisierten Patienten kommt als schonendstes Verfahren der Fixateur extern, dem DC-Konzept entsprechend zum Einsatz. Allerdings sollte innerhalb von 1-2 Wochen aufgrund der Gefahr der Pin-Infekte ein Verfahrenswechsel durchgeführt werden (RÜEDI et al. 2003, RIXEN et al. 2005 a).

Nur in ca. $6 \%$ der Fälle der Femurfrakturen handelt es sich um distale Femurfrakturen. Bei diesen Frakturen kommt es oftmals zu einem beträchtlichen Weichteilschaden oder es sind häufig offene Frakturen. Bei polytraumatisierten Patienten sowie offenen Frakturen mit schwerem Weichteilschaden kommt primär zur temporären Stabilisierung der gelenkübergreifende Fixateur extern zur Anwendung. Bei den intraartikulären distalen Femurfrakturen sollte die anatomische Rekonstruktion der Gelenkfläche Ziel der Osteosynthese sein. Es gibt hierzu verschiedene Fixationstechniken: interfragmentäre Kompression mittels Zugschrauben oder Defektüberbrückung mit Kortikalisschrauben, Kondylenplatte $95^{\circ}$, dynamische Kondylenschraube (DCS). Die Kondylenabstützplatte kam früher für komplexe Frakturen (33-C3) zum Einsatz, ist jedoch heute vom LISS, Fixateur intern, abgelöst. Für extraarticuläre einfache Frakturen kommt auch die retrograde Nagelung mittels solidem distalen Femurnagel (DFN) in Frage (RÜEDI et al. 2003).

\subsection{Fragestellung der Untersuchung}

Anhand einer retrospektiven Studie auf der Grundlage des Göttingen Polytraumaregisters sollte für den Untersuchungszeitraum September 1994 bis 


\section{Einleitung}

Dezember 2000 das Patientengut mit Oberschenkelfrakturen bei erlittenem Polytrauma untersucht und ausgewertet werden.

Es wurde dabei zu folgenden Fragen in der Auswertung Stellung genommen:

- Wie wirken sich Schweregrad der Verletzung und Verletzungskombinationen hinsichtlich des Outcomes des Polytraumapatienten aus?

- Welchen Einfluss haben der Zeitpunkt der Frakturversorgung und die Wahl der operativen Methode auf das Behandlungsergebnis ?

- Wie sind die Ergebnisse der sozialen und beruflichen Rehabilitation im untersuchten Patientengut? 
2. Methoden und Material

\subsection{Datenerfassung}

Die in der vorliegenden Arbeit verwendeten Daten sind Bestandteil der Polytraumastudie der Abteilung Unfallchirurgie, Plastische und Wiederherstellungschirurgie der Universitätsmedizin Göttingen. Die Datenerfassung und -analyse erfolgte im Zeitraum 01/2004 bis 07/2006. Es wurden hierbei Daten der Patienten erfasst, die im Zeitraum zwischen 01.09.1994 bis 31.12.2000 über den Schockraum der Universitätsmedizin Göttingen aufgenommen worden sind. In einer speziell für die Studie entwickelten Datenbank wurde diese Daten retrospektiv erfasst. Das heißt, die Daten über den Unfallhergang sowie das präklinische Intervall wurden retrospektiv erfasst, die Daten über Diagnostik und Therapie im Schockraum wurden durch den diensthabenden Arzt/Ärztin prospektiv aufgezeichnet. Aus den vorhandenen Akten wurde folgende Daten entnommen : Alter, Geschlecht, AIS, ISS, Unfalldatum/-uhrzeit, Unfallhergang, GCS, Vitalparameter, Notrufeingang, Datum und Uhrzeit der Aufnahme in der Klinik, in der Klinik : Diagnostik wann?, was?, Laborparameter, Operationen mit jeweiligem Zeitpunkt, Beatmungsdauer, Aufenthaltsdauer auf der Intensivstation und der Normalstation, Entlassungszeitpunkt.

\subsection{Einschlusskriterien}

Aus den o.g. in der Datenbank erfassten Patienten wurden für die vorliegende Arbeit jene ausgewählt, welche folgende Kriterien erfüllten:

1. Pol ytraumapatienten, d.h. wie in der Einleitung erläutert, ISS $\geq 16$

2. allzeitpunkt zwischen 01.09.1994 bis 31.12.2000

3. gnose Femurfraktur

Dia

4. $r \geq 16$ Jahre.

Alte

Bereits vorhandene Daten und noch fehlende Daten, der durch die o.g. Kriterien herausgefilterten Patienten, wurden anhand der Angaben aus den Krankenakten 
überprüft bzw. ergänzt. Insbesondere der errechnete ISS (Injury Severity Score) zwecks der Einteilung der Verletzungsschwere wurde von der Autorin und zwei unabhängigen Untersuchern nochmals überprüft. Für die Diagnosesicherung Femurfraktur wurden die vorhandenen Röntgenbilder gesichtet, digitalisiert und an Hand der Kriterien der „Arbeitsgemeinschaft für Osteosynthesefragen“ (AO) klassifiziert. Die durch die Untersucherin klassifizierten Bilder wurden von 2 unabhängigen Untersuchern nochmals im Hinblick auf die Einteilung überprüft.

Um mögliche Fehler in der Datenerfassung zu vermeiden, wurden die Daten nach einem zeitlichen Abstand von ca. einem Monat nochmals überprüft, des weiteren wurden Patientenakten und Röntgenbilder, welche im Archiv nicht vorrätig waren, in zeitlichen Abständen mindestens drei weitere Male bestellt.

\subsection{Nachuntersuchung der ausgewählten Patienten}

Alle Patienten, die die o.g. Einschlusskriterien erfüllten, wurden persönlich angeschrieben und zu der geplanten Nachuntersuchung eingeladen. Es erfolgte hiernach telefonische Absprache mit dem jeweiligen Patienten bzw. Patientin zwecks Terminvereinbarung. Patienten, die nicht zu einer Nachuntersuchung in die Universitätsmedizin Göttingen kommen konnten, erhielten mit dem Anschreiben gleichzeitig den von der Doktorandin erstellten Fragebogen (im Anhang) zur Beantwortung mit beiliegendem frankierten Rückumschlag. Alle Patienten wurden dahingehend informiert, dass die Teilnahme an der Nachuntersuchung freiwillig im Rahmen einer Dissertationsarbeit erfolgt, und dass bei Nichtteilnahme innen keinerlei Nachteile für die Weiterbehandlung entstehen. Ihnen wurde des weiteren mitgeteilt, dass die Daten anonymisiert ausgewertet werden.

Der entwickelte Fragebogen enthielt zunächst 20 Fragen. Er wurde von 12 gesunden Probanden, welche keinen Bezug zur Polytraumastudie hatten, validiert. Die Probanden bestanden aus 7 Männern und 5 Frauen, der Altersdurchschnitt lag bei $22,7 \pm 3,5$ Jahren. Der bei der Untersuchung angewandte Fragebogen enthielt nach Überarbeitung 15 Fragen. 
Patienten, die nach telefonischer Terminvereinbarung bereit waren, zu einer Nachuntersuchung in die Universitätsmedizin Göttingen zu kommen, erhielten vor der Untersuchung den o.g. Fragebogen ausgehändigt, um ihn eigenständig auszufüllen. Die Nachuntersuchung diente der Erfassung der subjektiven Beschwerden der Patienten sowie der gezielten klinischen Untersuchung.

Um eine standardisierte vergleichbare Untersuchung zu ermöglichen, wurde vorab eine Untersuchungsbogen (im Anhang) erstellt, dessen einzelne Fragestellungen bzw. Punkte bei jedem Patienten abgearbeitet wurden. Der Untersuchungsbogen wurde auf die anatomischen und funktionellen Gesichtspunkte bei einer Oberschenkelverletzung ausgerichtet. Die Nachuntersuchungen erfolgten in den Untersuchungsräumen der Abteilung Unfallchirurgie, Plastische und Wiederherstellungschirurgie der Universitätsmedizin Göttingen unter Aufsicht eines Assistenzarztes oder eines Facharztes für Unfallchirurgie. Auf eine erneute Röntgenkontrolle wurde zum Untersuchungszeitpunkt verzichtet, da in den meisten Fällen ausreichend postoperative Verlaufsbilder vorhanden waren bzw. die Patienten selbst extern angefertigte Bilder zur Untersuchung mitbrachten.

\subsection{Datenverarbeitung und Statistik}

Die Datenerfassung und Bearbeitung erfolgte mit FileMaker Pro 4.0 auf einem Apple Macintosh Computer. Über diese relationale Datenbank war es möglich, beliebig verschiedene Daten, welche zuvor erfasst waren, zu suchen und sie, wenn nötig den jeweiligen Patienten zuzuordnen.

Zur weiteren Datenanalyse wurden die erfassten Daten anonymisiert. Wie bereits in der Einleitung erläutert, wurden die Entlassungsdiagnosen nach dem Abbreviated Injury Scale (AIS) von jeweils 0-5 kodiert, woraus sich dann der Injury Severity Score (ISS) errechnete. Die statische Auswertung der Daten erfolgte mit Hilfe des Computerprogrammes Statistica for Windows ${ }^{\circledR}$ 6.0. Mit Hilfe der „deskriptiven Statistik“ ließen sich die verschiedenen Verteilungen der Parameter sowie besondere Auffälligkeiten beschreiben. Ergänzende graphische Darstellungen wurden mit Hilfe des Programms Microsoft Excel für Windows ${ }^{\circledR}$ erstellt. Zur statistischen Auswertung der zwei Patientengruppen wurden für dichotome Variablen der Chi $^{2}$-Vierfeldertest und 
für analoge Skalen der Wilcoxon-Mann-Whitney-Test verwendet. Das Signifikanzniveau lag bei den beiden Tests jeweils bei $5 \%$ (zweiseitiges $\alpha$ ). Die folgenden Parameter der Auswertung der Ergebnisse werden im weiteren Teil dieser Arbeit angegeben: Prozentanteil des Gesamtkollektivs, Mittelwert mit Standardabweichung, Minimum und Maximum, Medianwert, 25- und 75\%-Quartile.

\subsection{Auswertung des Fragebogens und der klinischen Nachtuntersuchung}

Die vorhandenen Daten, der von den Patienten bearbeiteten Fragebögen, wurden ebenfalls mittels Statistica for Windows ${ }^{\circledR}$ ausgewertet. Sollten bei der Beantwortung der einzelnen Fragen mehrere Ziffern angekreuzt worden sein, wurde jeweils die kleinste Ziffer gewertet, sofern durch schriftliche Anmerkungen des Patienten nicht ein Kreuz auf logischem Weg ausgeschlossen werden konnte.

Zur weiteren Auswertung der Ergebnisse der Untersuchung - ergänzend durch den beantworteten Fragebogen - erfolgte die Erstellung eines Beurteilungsschemas (im Anhang) in Anlehnung an den Neer-Score (NEER et al. 1967). Bei diesem Beurteilungsschema dienten die Einflussgrößen „Schmerz, Funktion und Arbeitsfähigkeit“ als Grundlage. Da in der hier vorliegenden Arbeit die Lebensqualität und nicht das radiologische Ergebnis im Vordergrund standen, wurden die einzelnen Einflussgrößen anders gewichtet. Ein sehr gutes Ergebnis zeigten die Patienten mit einer Punktzahl von 68-85, ein gutes mit einer Punktzahl von 47-67, ein mäßiges Ergebnis mit einer Punktzahl von 22-46, diejenigen mit einer Punktzahl von 0-21 ein nicht zufriedenstellendes Ergebnis.

Die vorliegende Studie im Rahmen der Göttinger Polytraumastudie wurde von der Ethikkommission der Universitätsmedizin der Georg-August-Universität zu Göttingen genehmigt (Antragsnummer 28/1/03).

\subsection{Definitionen}

\section{$\underline{\text { Unterteilung der Patienten mit Femurfraktur }}$}


Gruppe 1 : Patienten mit frühzeitiger operativer Versorgung der Femurfraktur, d.h. Operationsbeginn innerhalb der ersten 6 Stunden (360 Minuten) nach Aufnahme in der Klinik.

Gruppe 2 : Patienten mit verzögerter operativer Versorgung der Femurfraktur, d.h. Operationsbeginn erst nach den ersten 6 Stunden (360 Minuten) nach Aufnahme in der Klinik.

Intensivdauer : Zeitliche Dauer zwischen Aufnahme auf der Intensivstation und Verlegung auf Normalstation.

Stationäre Behandlungsdauer : Zeitdauer des gesamten Aufenthalts des Patienten in der Universitätsmedizin Göttingen.

Beatmungsdauer : Zeitspanne zwischen Intubation und Extubation, sollte ein Patient mehrfach intubiert gewesen sein, wurden die jeweiligen Zeiten addiert. 


\section{Ergebnisse}

\section{1. Überblick über die Schockraumpatienten}

Über den Schockraum der Universitätsmedizin Göttingen wurden im Zeitraum vom 01.09.1994 bis zum 31.12.2000 2285 Patienten aufgenommen, 684 von innen konnten innerhalb des Studienzeitraumes als polytraumatisiert identifiziert werden, wie aus der nachfolgenden Abbildung erkennbar ist.

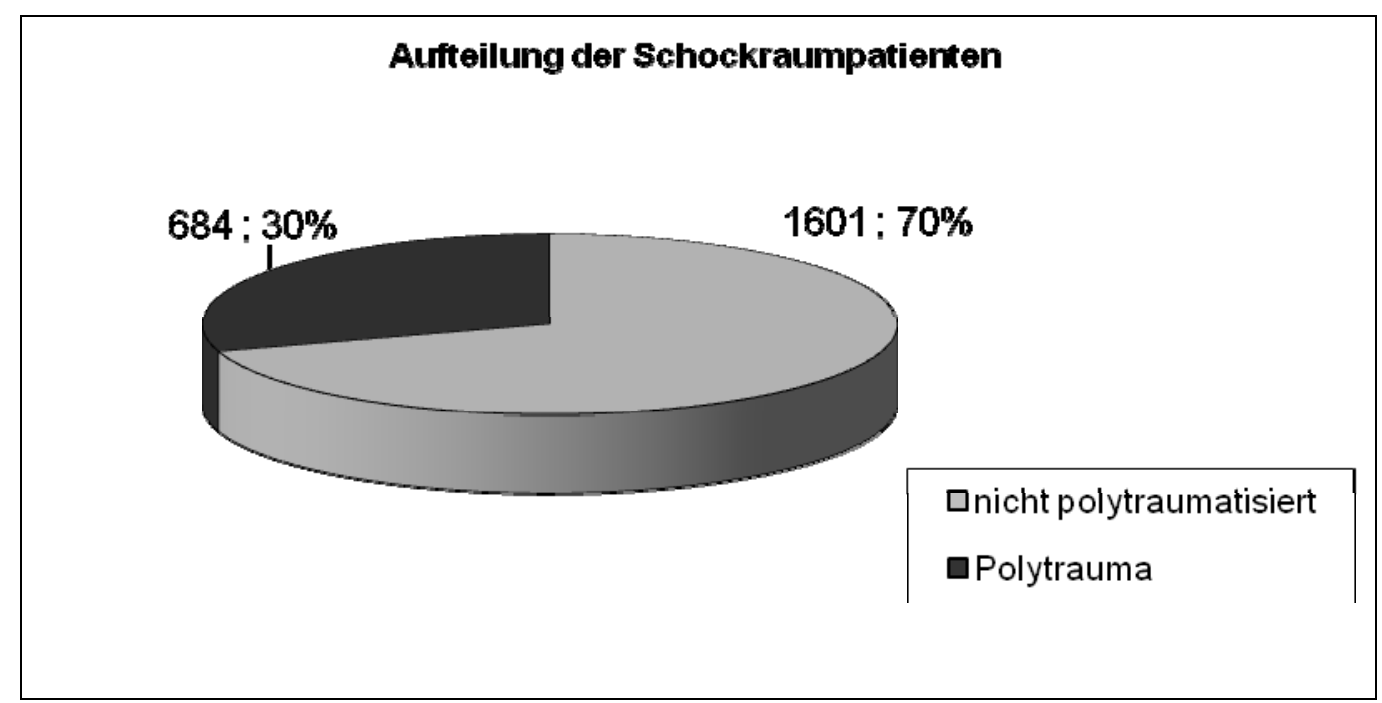

Abbildung 3: Aufteilung der Schockraumpatienten

(Polytrauma versus nicht polytraumatisierte Patienten).

Die Verteilung der Patienten auf die einzelnen Jahre ist der folgenden Abbildung zu entnehmen, hierbei ist zu beachten, dass im Jahre 1994 eine deutlich geringere Anzahl Patienten im Schockraum aufgenommen wurde, dies ist jedoch erklärbar durch den Beginn der Dokumentation, die erst ab September des Jahres begann. Die Schockraumpatientenanzahl schwankte in den Jahren 1995 bis 2000 zwischen $488 \mathrm{im}$ Jahre 2000 und 252 im Jahre 1999. Bei der Geschlechterverteilung der aufgenommen Patienten zeigt sich mit Ausnahme des Jahres 1996, dass überwiegend männliche Patienten im Schockraum aufgenommen wurden. Unter den polytraumatisch verletzten Patienten schwankte die Verteilung auf die einzelnen Jahre zwischen 123 1995 und 100 Patienten 1996. Die Schockraumpatienten setzten sich insgesamt aus 1360 (59,52\%) männlichen Patienten und 925 (40,48\%) weiblichen Patienten zusammen, bei den davon polytraumatisierten Patienten waren es $480(70,18 \%)$ männliche und 204 (29,82\%) weibliche Patienten. 


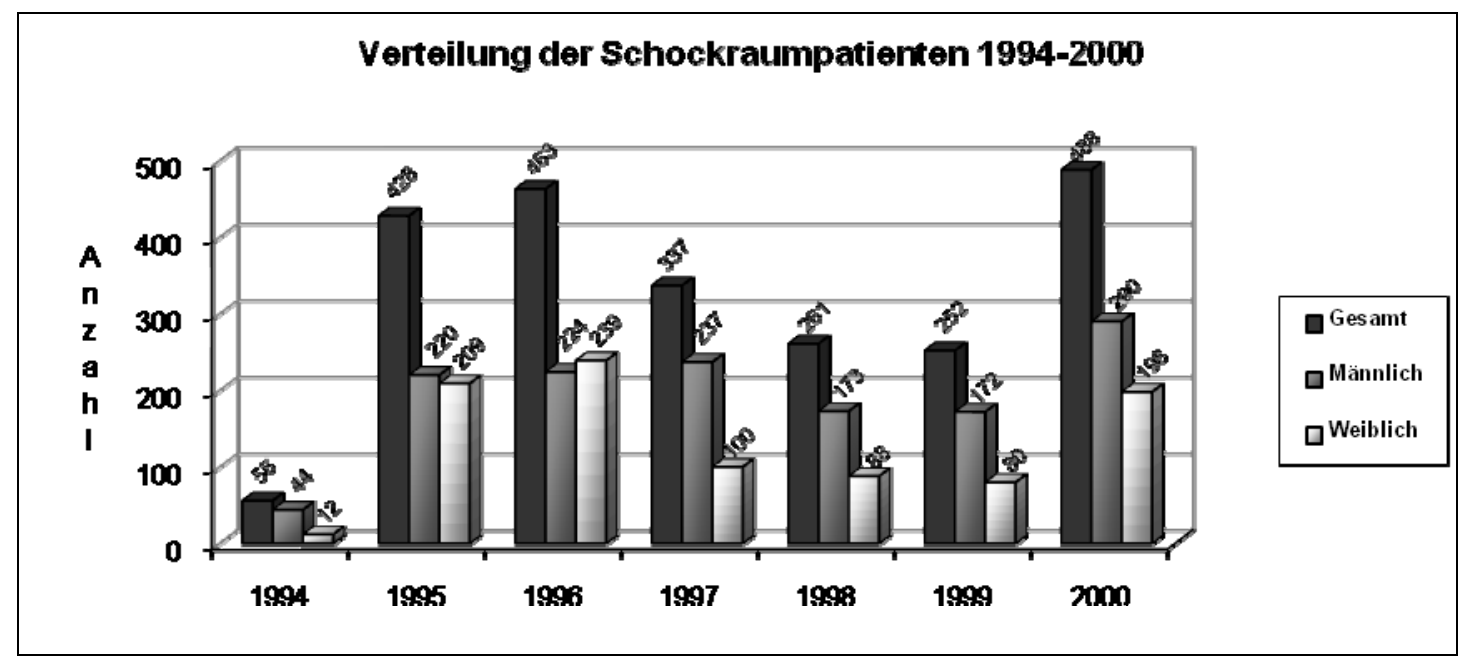

Abbildung 4: Verteilung der Schockraumpatienten auf die Jahre 1994-2000.

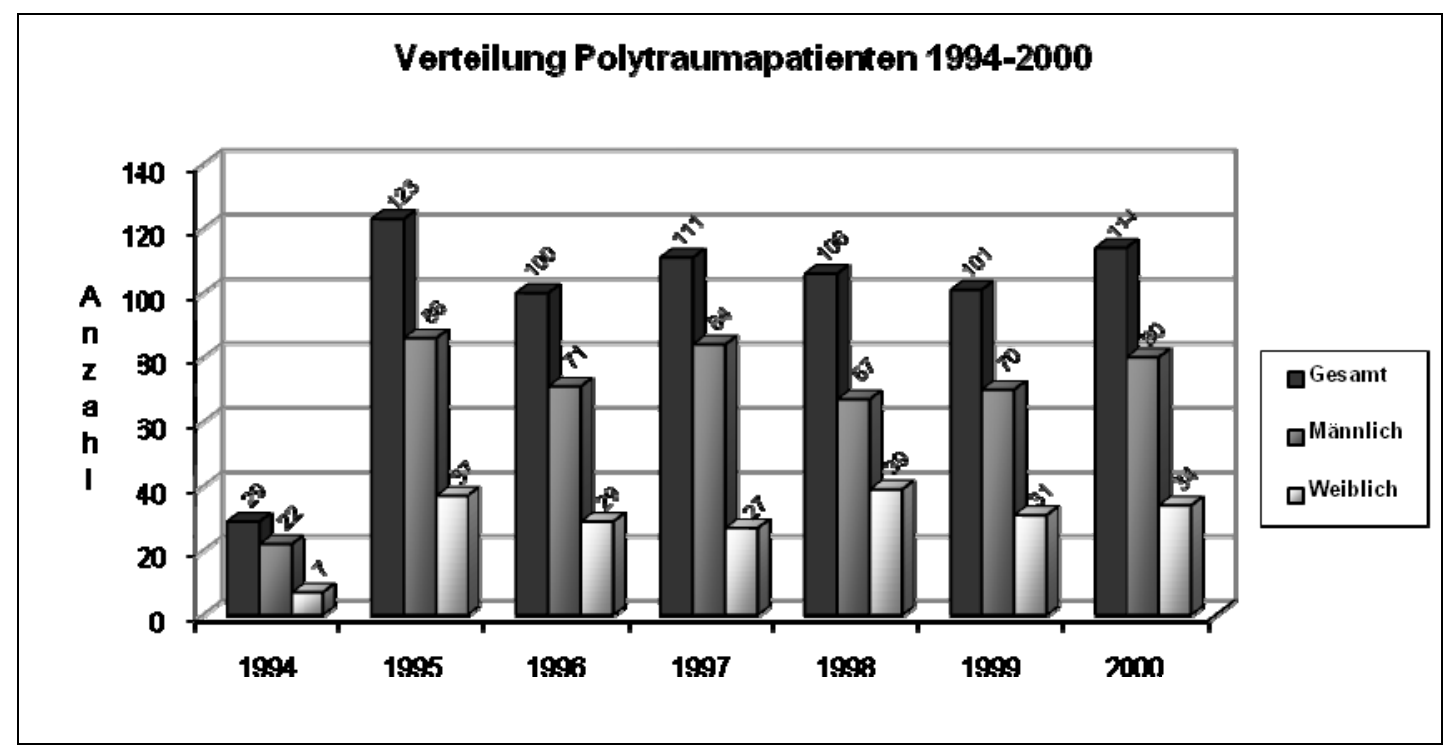

Abbildung 5: Verteilung der Polytraumapatienten auf die Jahre 1994-2000.

\subsection{Gesamte Polytraumastudie}

Von den 684 polytraumatisch verletzten Patienten, die in den Jahren 1994-2000 über den Schockraum der Universitätsmedizin Göttingen aufgenommen wurden, überlebten 563 Patienten (82,31\%), 121 Patienten (17,69\%) verstarben im weiteren Verlauf. In der nachfolgenden Abbildung ist erkennbar, dass die Haupttodesursache unabhängig vom Todeszeitpunkt das Schädelhirntrauma ist, gefolgt von Massenblutung im Abdomen oder Thorax. Dagegen spielen erst im weiteren zeitlichen Verlauf Thoraxtrauma und Beckenverletzung, sowie Organverletzungen eine Rolle. 


\section{Ergebnisse}

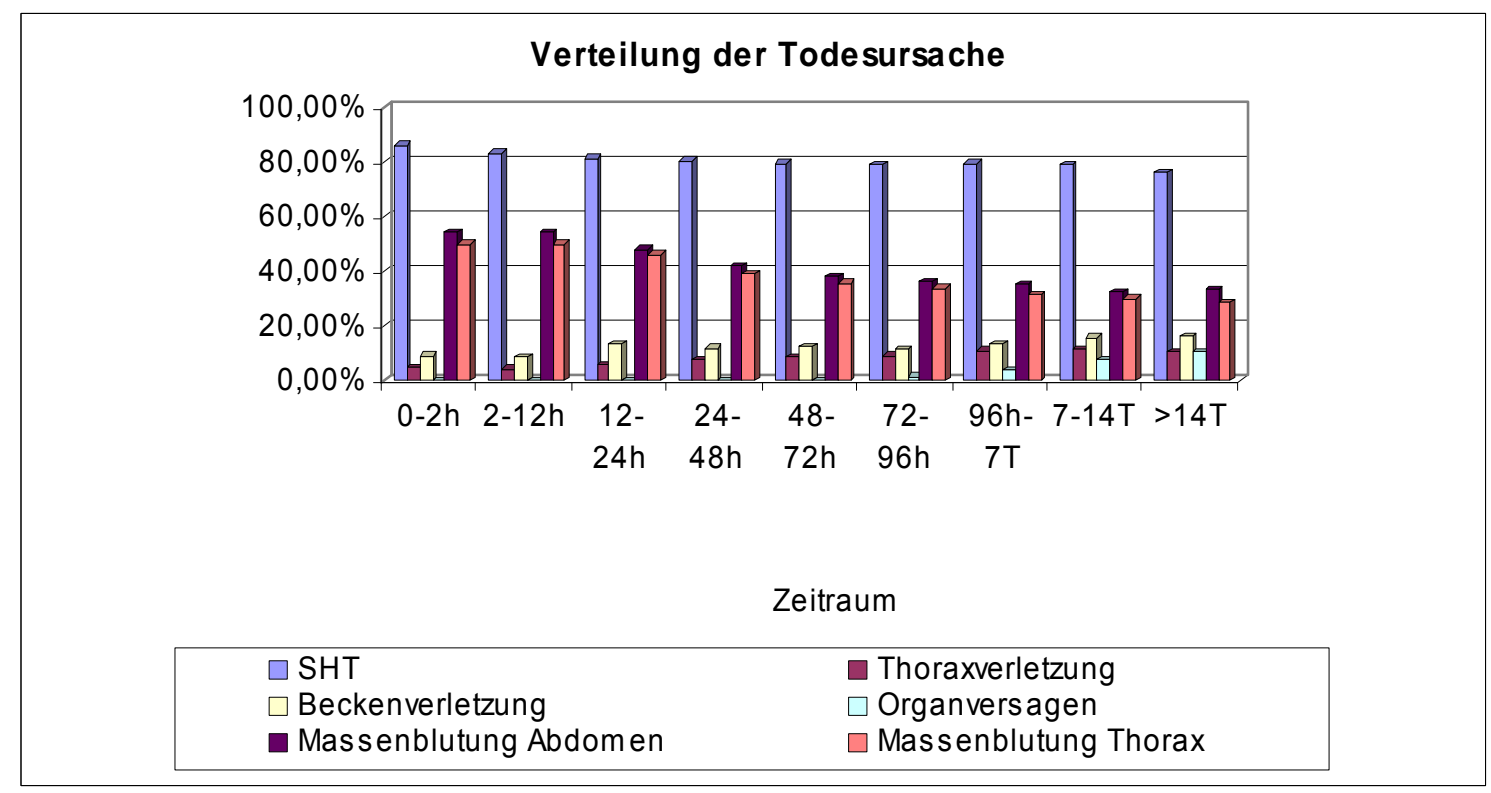

Abbildung 6: Verteilung der Todesursache nach Todeszeitpunkt.

\subsubsection{Abbreviated Injury Scale (AIS)}

Wie bereits in der Einleitung erläutert, wurde bei allen Patienten jeder Einzelverletzung ein bestimmter AIS-Wert und ebenso je nach Lokalisation eine Körperregion zugeordnet. Im Folgenden sind nun die AIS-Mittelwerte mit Standardabweichung in den einzelnen Regionen dargestellt.

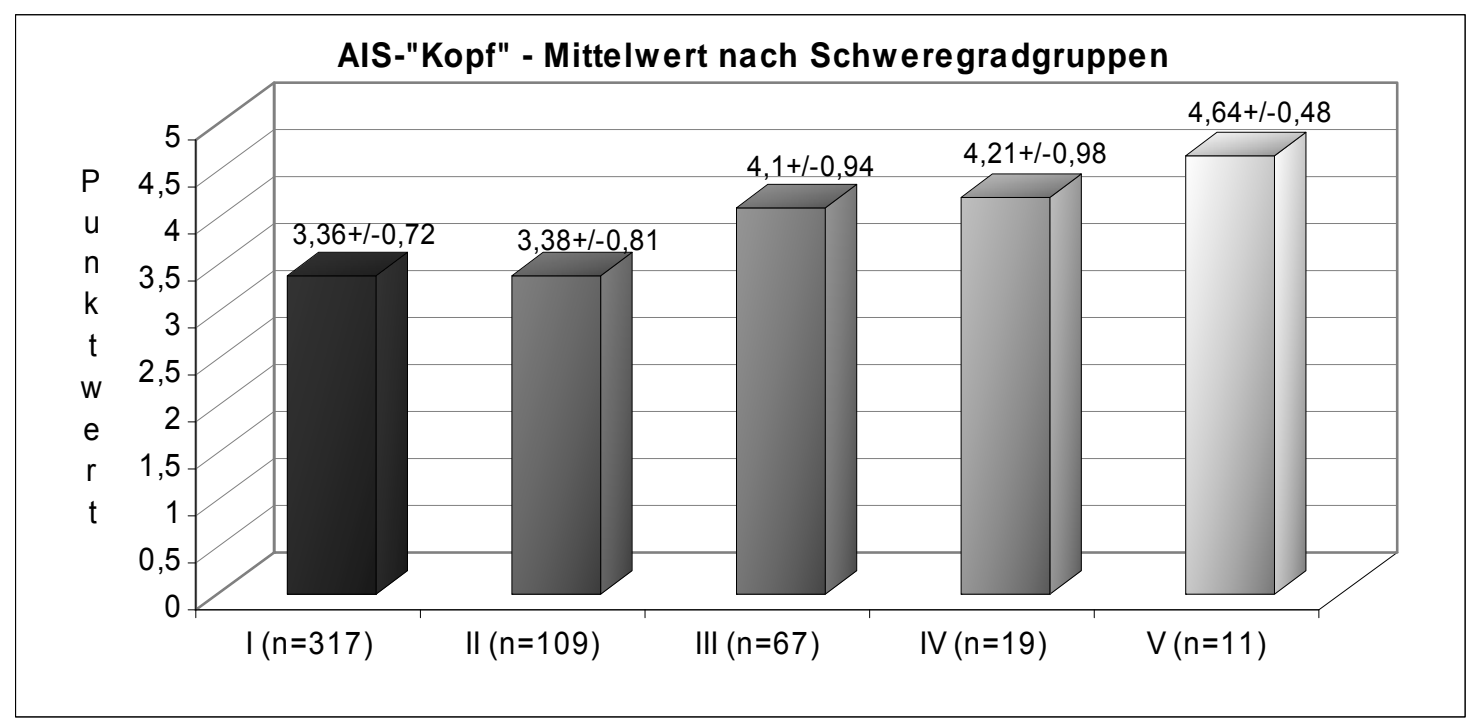

Abbildung 7: AIS-,Kopf“-Mittelwert nach Schweregradgruppen unterteilt. 


\section{Ergebnisse}

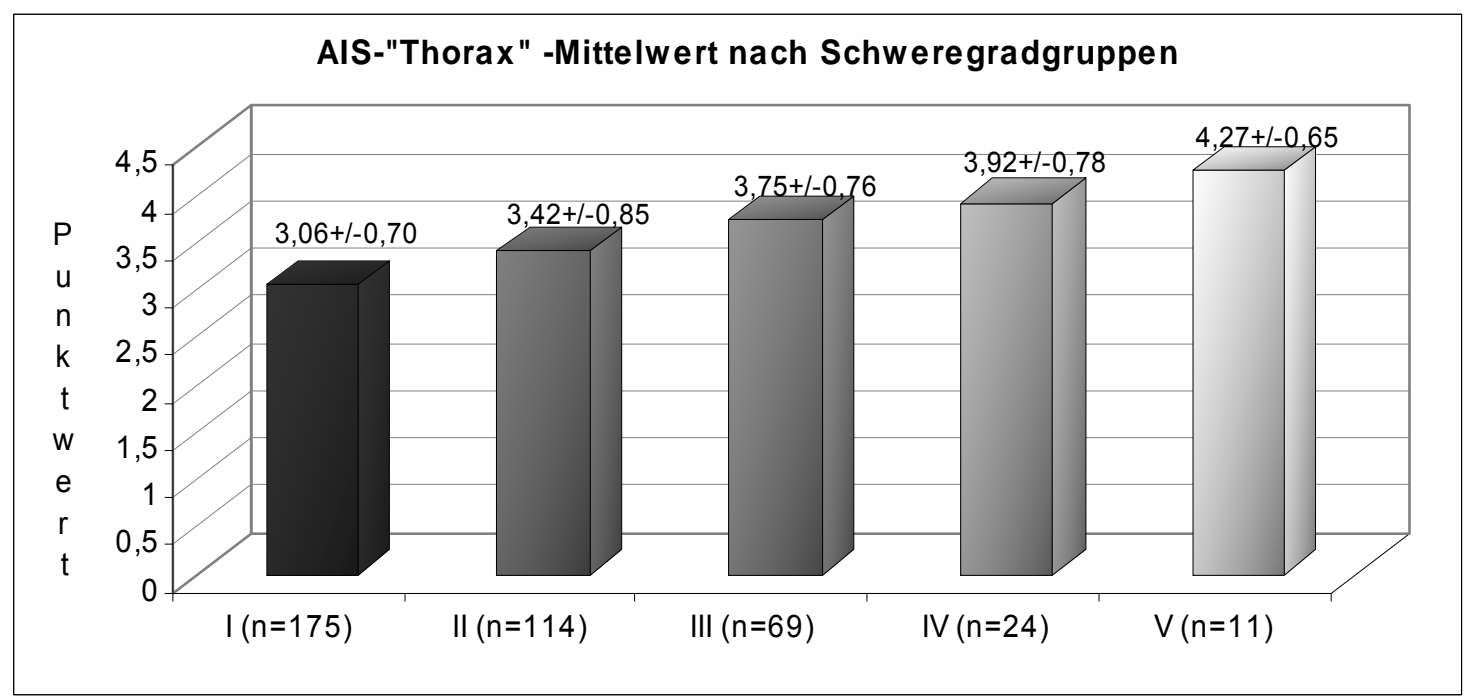

Abbildung 8: AIS-„,Thorax“-Mittelwert nach Schweregradgruppen.

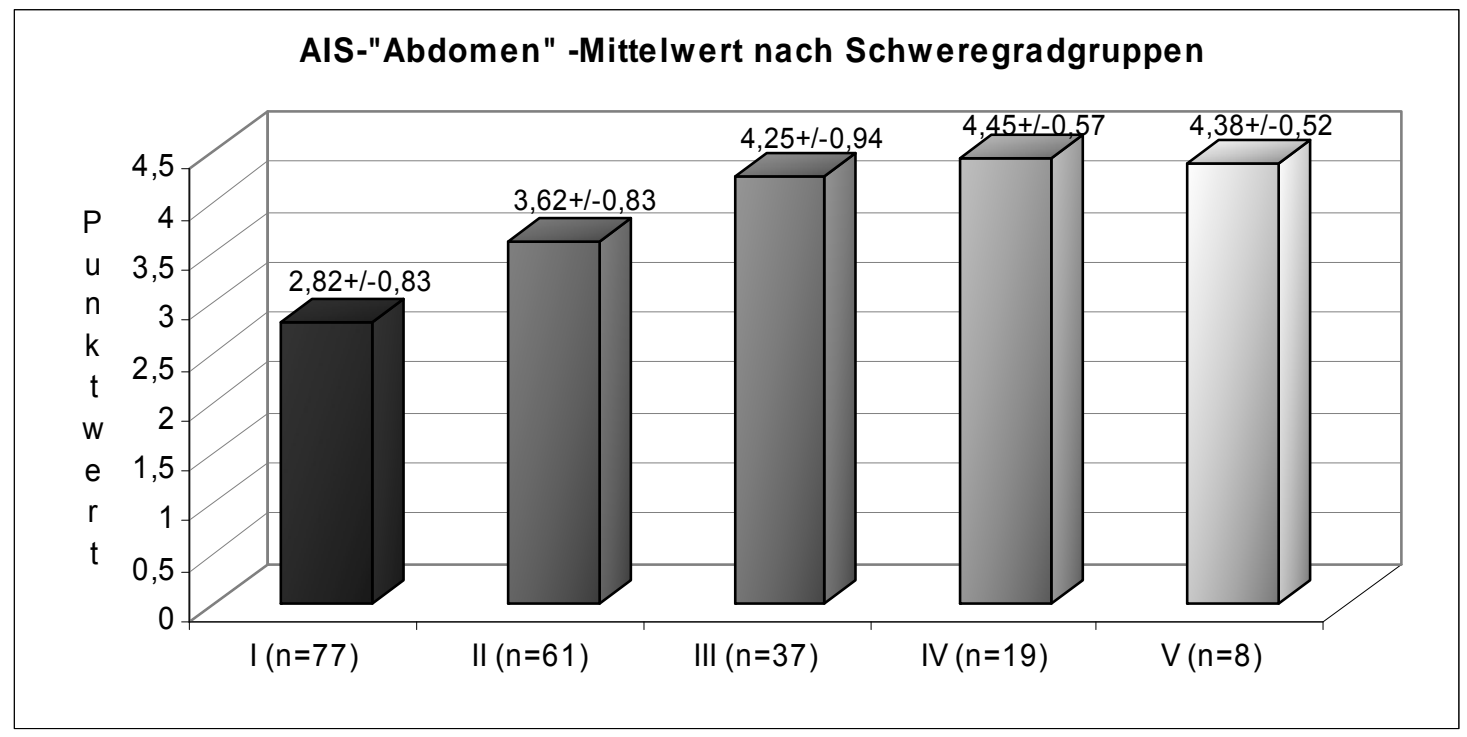

Abbildung 9: AIS-„Abdomen“-Mittelwert nach Schweregradgruppen.

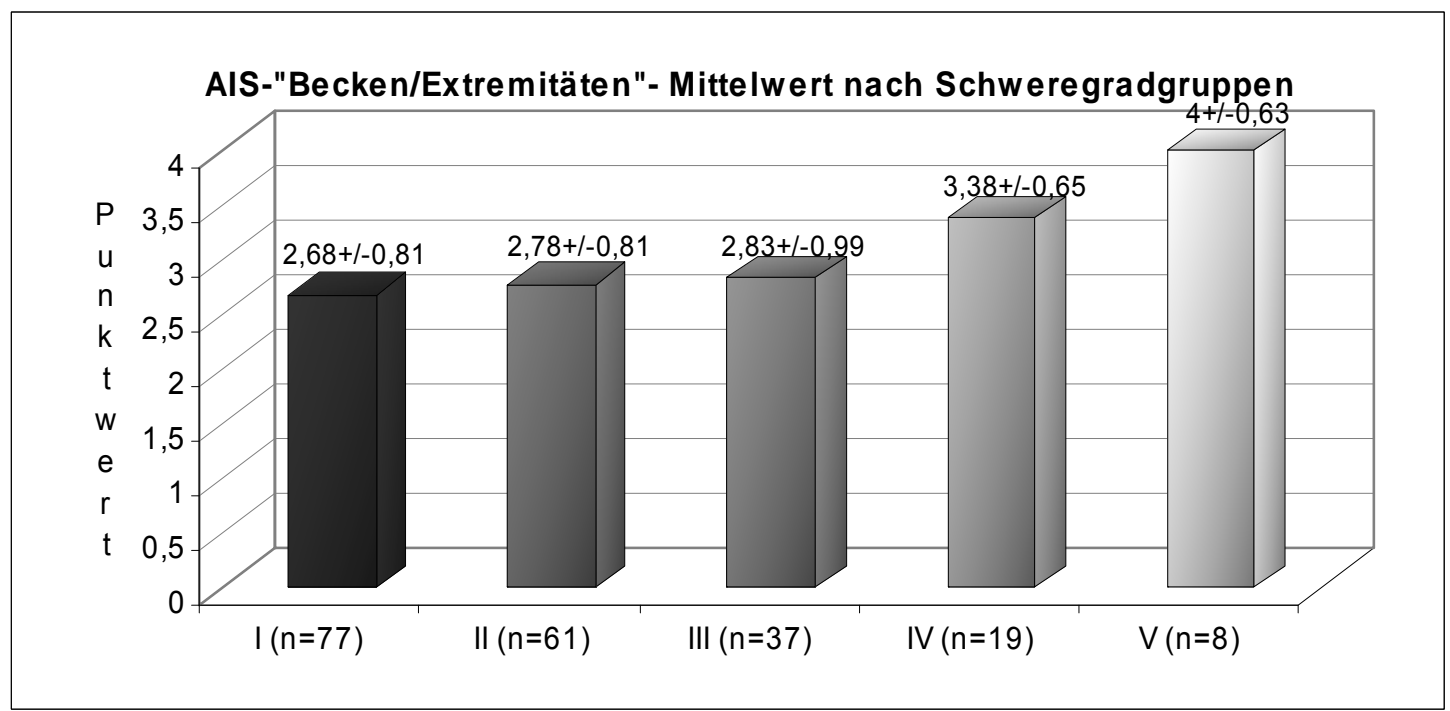

Abbildung 10: AIS-,Becken/Extremitäten“-Mittelwert nach Schweregradgruppen. 
523 Patienten $(76,5 \%)$ der 684 Polytraumapatienten hatten eine Verletzung im Kopfbereich. Dies war die am häufigsten betroffene Region. Der AIS-Mittelwert „Kopf“ betrug 3,52 $\pm 1,15$. Eine Verletzung im Thoraxbereich zogen sich 393 Patienten $(57,5 \%)$ zu, hierbei betrug der AIS-Mittelwert „Thorax“ 3,38 $\pm 0,83$. Der geringste Anteil betraf die Region des Abdomen, hier verletzten sich 202 Patienten (29,5\%). Einen ebenfalls großen Anteil stellten die Verletzungen des Beckens und der Extremitäten mit 444 betroffenen Patienten (64,9\%). Wobei der AIS-Mittelwert „Becken/

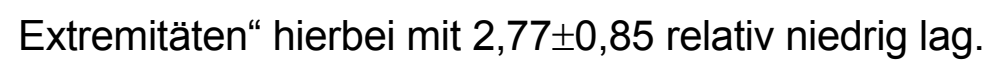

\subsection{Gesamtpolytraumastudie versus Polytraumapatienten mit Femurfraktur}

Daten von 684 polytraumatisch verletzten Patienten, die in den Jahren 1994-2000 über den Schockraum der Universitätsmedizin Göttingen aufgenommen wurden, konnten ausgewertet werden. Aus dem genannten Kollektiv wurden 91 Patienten mit Femurfraktur detailliert untersucht.

\subsubsection{Jahresverteilung}

In der folgenden Abbildung ist die Jahresverteilung für die Fälle der Gesamtpolytraumastudie im Vergleich zu den Fällen des Polytraumas mit Femurfraktur aufgezeigt. Einschränkungen bestehen für das Jahr 1994, dass durch den Beginn der Studie nur zu einem Drittel erfasst werden konnte.

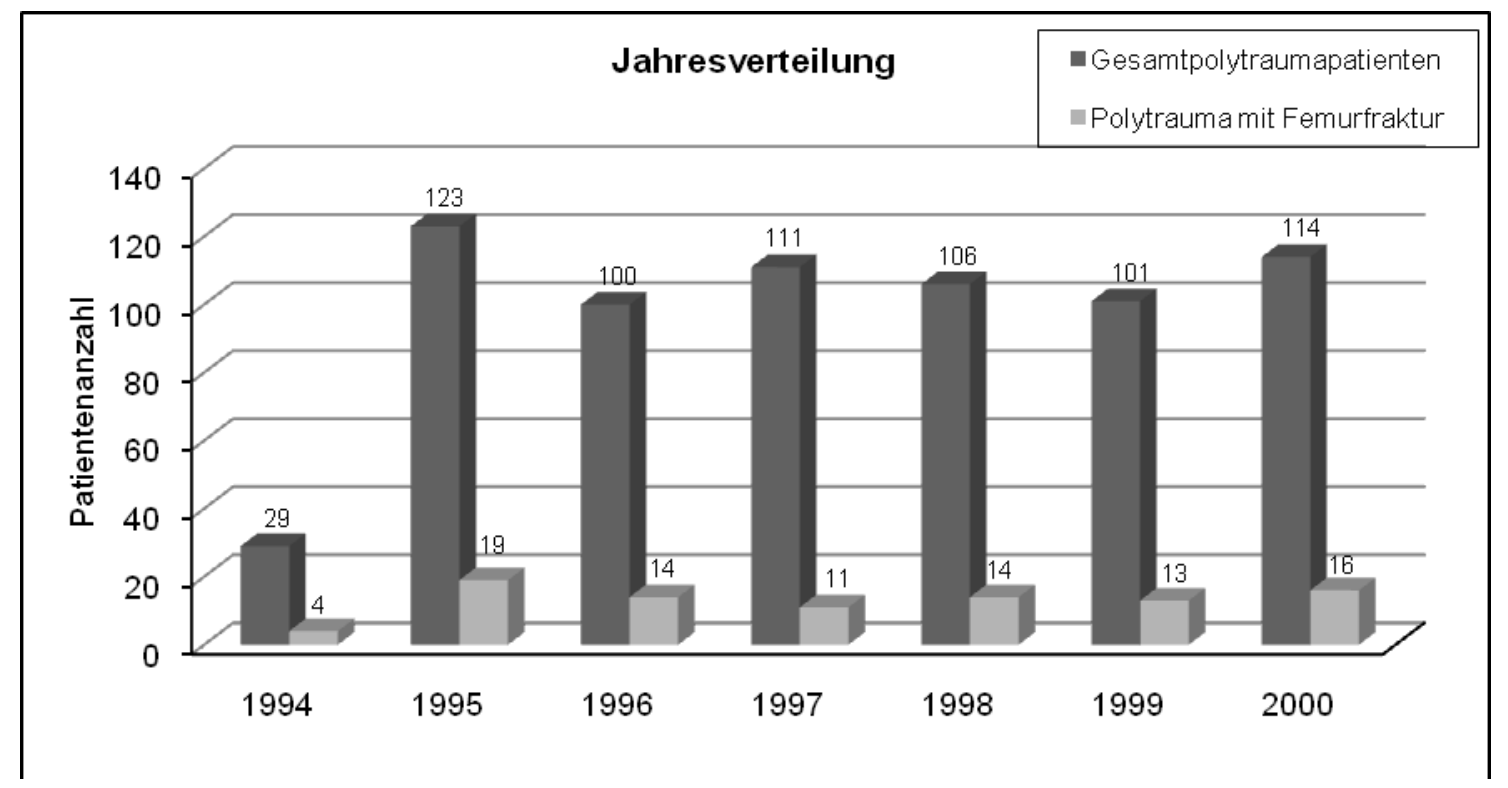

Abbildung 11: Jahresverteilung. 


\subsubsection{Geschlechtsverteilung}

Die Geschlechtsverteilung in der Gruppe mit Femurfraktur ist im Vergleich zum Gesamtkollektiv der Polytraumapatienten etwa gleich. Von 684 polytraumatisierten Patienten waren 480 (70\%) männlichen Geschlechts und 204 (30\%) weiblichen Geschlechts. In der Gruppe der Polytraumatisierten mit Femurfraktur waren 27 (30\%) weiblichen und 64 (70\%) männlichen Geschlechts, wie in den folgenden Abbildungen zu sehen ist.

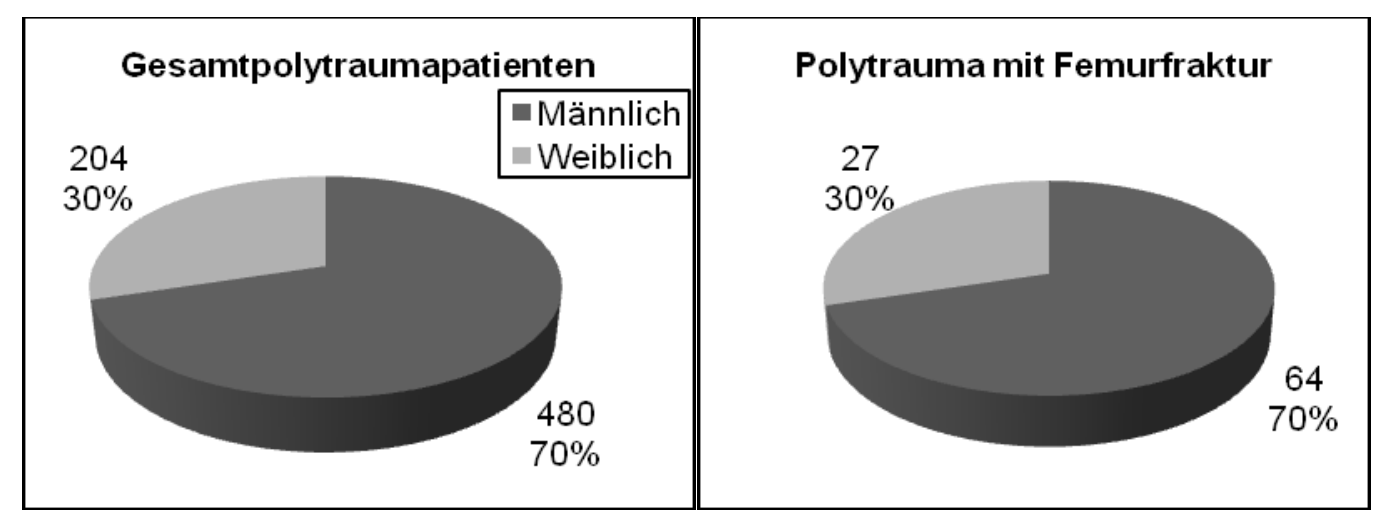

Abbildung 12/13: Geschlechtsverteilung Gesamtpolytraumapatienten und Polytrauma- patienten mit Femurfraktur.

\subsubsection{Alter- und Geschlechtsverteilung nach Schweregradgruppierung}

Entsprechend dem ISS wurden die Patienten wie in Punkt 3.2.1. in verschiedene Schweregradgruppen eingeteilt : Gruppe I (ISS 16-25), Gruppe II (ISS 26-35), Gruppe III (ISS 36-45), Gruppe IV (ISS 46-55), Gruppe V (ISS $\geq 55$ ).

Zum Zeitpunkt des Unfalles lag das Durchschnittsalter aller Polytraumapatienten bei $39,7 \pm 21,0$ Jahren.

Bei den Polytraumapatienten mit Femurfraktur lag im Vergleich hierzu, zum Zeitpunkt des Unfalles, das Durchschnittsalter bei 34,95 $\pm 16,15$ Jahren. Die entsprechenden Alters- und Geschlechtsverteilungen sind den nachfolgenden Tabellen zu entnehmen. 
Tabelle 5: Alters- und Geschlechtsverteilung der Polytraumapatienten nach Schweregradgruppen, sowie Überlebenden und Verstorbenen unterteilt.

\begin{tabular}{|c|c|c|c|c|c|}
\hline & \multirow[t]{2}{*}{ Anzahl $(\mathrm{N}=)$} & \multicolumn{2}{|c|}{ ALTER } & \multicolumn{2}{|c|}{ GESCHLECHT } \\
\hline & & Mittelwert & $\begin{array}{l}\text { Standard- } \\
\text { abweichung }\end{array}$ & Männer & Frauen \\
\hline $\begin{array}{l}\text { Gesamtpoly- } \\
\text { traumapatienten }\end{array}$ & 684 & 39,7 & 21,0 & 480 & 204 \\
\hline ISS 16-25 & 412 & 40,8 & 21,1 & 289 & 123 \\
\hline ISS 26-35 & 150 & 40,0 & 20,1 & 110 & 40 \\
\hline ISS 36-45 & 85 & 35,8 & 20,4 & 56 & 29 \\
\hline ISS 46-55 & 25 & 37,5 & 21,6 & 18 & 7 \\
\hline ISS $\geq 55$ & 12 & 31,3 & 12,8 & 6 & 6 \\
\hline Überlebende & 563 & 38,4 & 19,9 & 401 & 162 \\
\hline Verstorbene & 121 & 46,0 & 24,4 & 79 & 42 \\
\hline
\end{tabular}

Tabelle 6: Alter und Geschlechtsverteilung der Polytraumapatienten mit Femurfraktur nach Schweregradgruppierung sowie Überlebenden und Verstorbenen unterteilt.

\begin{tabular}{|l|c|c|c|c|c|}
\hline & \multicolumn{1}{|l|}{$\begin{array}{l}\text { Anzahl } \\
(\mathrm{N}=)\end{array}$} & \multicolumn{2}{|c|}{$\begin{array}{l}\text { ALTER } \\
\text { Mittelwert }\end{array}$} & $\begin{array}{l}\text { Standard- } \\
\text { abweichung }\end{array}$ & $\begin{array}{l}\text { GESCHLECHT } \\
\text { Männer }\end{array}$ \\
\hline $\begin{array}{l}\text { Polytraumapatienten } \\
\text { mit Femurfraktur }\end{array}$ & 91 & 34,95 & 16,15 & 64 & 27 \\
\hline ISS 16-25 & 44 & 36,90 & 17,75 & 27 & 17 \\
\hline ISS 26-35 & 31 & 31,74 & 13,42 & 23 & 8 \\
\hline ISS 36-45 & 9 & 36,44 & 16,88 & 8 & 1 \\
\hline ISS 46-55 & 5 & 35,80 & 20,38 & 4 & 1 \\
\hline ISS $\geq 55$ & 2 & 34,00 & 8,48 & 2 & 0 \\
\hline Überlebende & 80 & 34,46 & 16,36 & 54 & 26 \\
\hline Verstorbene & 11 & 38,54 & 14,68 & 10 & 1 \\
\hline
\end{tabular}

\subsubsection{Todeszeitpunkt}

Unter den verstorbenen Patienten der Gesamtpolytraumastudie waren 79 männliche und 42 weibliche Patienten. Im Vergleich hierzu verstarben bei den Polytraumapatienten mit Femurfraktur 10 männliche Patienten und 1 weibliche Patientin.

In der nachfolgenden Abbildung ist die Patientenanzahl in Verbindung mit dem Todeszeitpunkt aufgeführt, hierbei zeigt sich, in unserer Gesamtpolytrauma-Studie, dass $18 \%$ der verstorbenen Patienten innerhalb der ersten 2 Stunden und 44,62 \% der insgesamt verstorbenen Patienten innerhalb der ersten 24 Stunden verstorben sind. In 
der Gruppe der Polytraumatisierten mit Femurfraktur verstarben 2 Patienten $(18,2 \%)$ bereits im Schockraum und 9 Patienten $(81,8 \%)$ während des weiteren stationären Aufenthaltes.

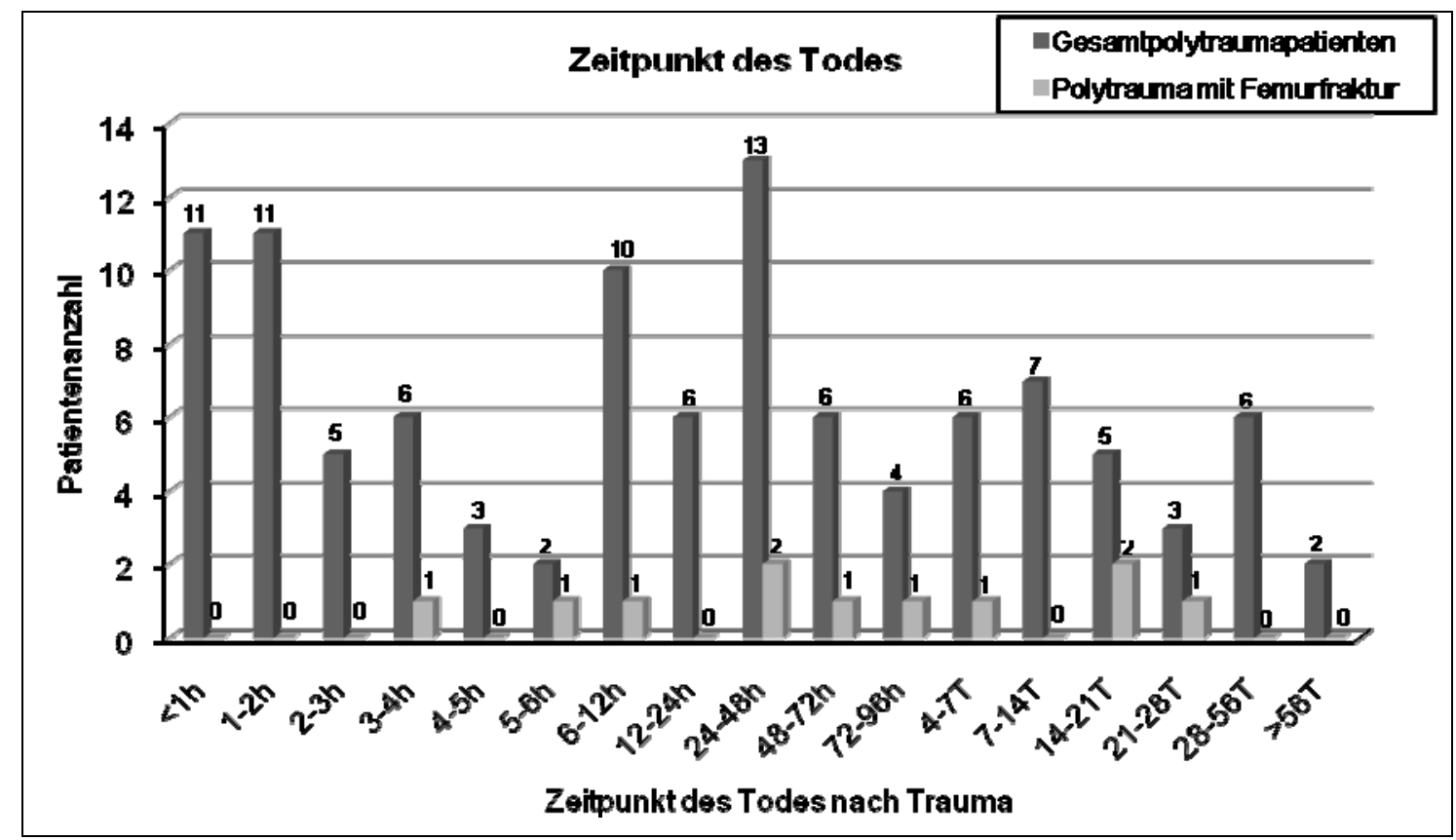

Abbildung 14: Zeitpunkt des Todes.

\subsubsection{Intensivstationsaufenthalts- und Beatmungsdauer}

Von 417 polytraumatisierten Patienten sowie 80 polytraumatisierten Patienten mit Femurfraktur konnte laut den Akten eine exakte Dauer für den Intensivstationsaufenthalt errechnet werden. Durchschnittlich befanden sich die

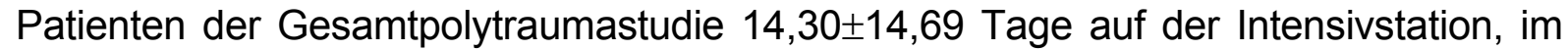
Vergleich hierzu befanden sich die Patienten mit Femurfraktur 15,35 $\pm 17,41$ Tage auf der Intensivstation. Aus der nachfolgenden Abbildung ist erkennbar, dass sich in der Gesamtpolytraumastudie mit zunehmender Verletzungsschwere erwartungsgemäß auch die Aufenthaltsdauer auf der Intensivstation verlängert mit Gruppe I 10,20 $\pm 10,62$ Tage bis Gruppe V mit 28,90 $\pm 26,10$ Tage. Dies trifft auch für die Schweregradgruppen I-III der Polytraumapatienten mit Femurfraktur zu, in Gruppe I betrug die Aufenthaltsdauer auf der Intensivstation 8,59 $\pm 10,01$ Tage, in Gruppe III 41,86 $\pm 30,21$ Tage. Für die Gruppen IV und V ist dies nicht nachweisbar, da die auswertbare 
Datenmenge hier zu gering ausfiel bzw. in der Gruppe $\mathrm{V}$ die beiden Patienten verstorben waren.

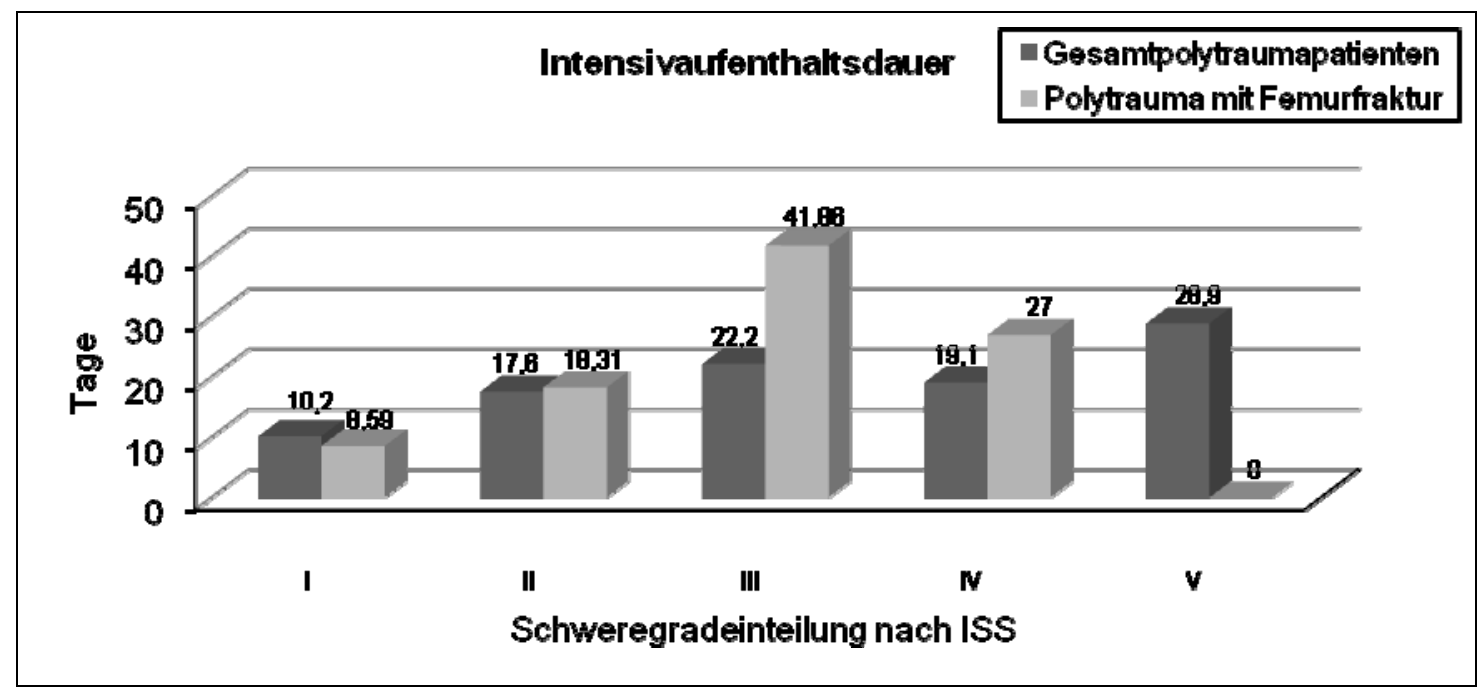

Abbildung 15: Intensivaufenthaltsdauer nach Schweregradgruppierung (Gruppe I (ISS 16-25), Gruppe II (ISS 26-35), Gruppe III (ISS 36-45), Gruppe IV (ISS 46-55), Gruppe V (ISS $\geq 55)$ ).

Die Durchschnittsbeatmungsdauer konnte bei 250 Patienten der Polytraumapatienten

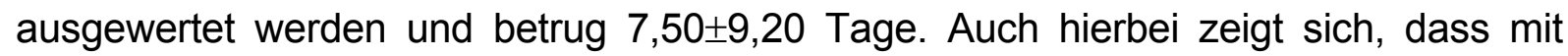
zunehmender Verletzungsschwere auch die Beatmungsdauer steigt, von 4,6 \pm 5 ,8 aus Gruppe I auf 20,0 $\pm 14,4$ Tage. Bei 79 Patienten der Polytraumapatienten mit Femurfraktur konnte die Durchschnittsbeatmungsdauer ausgewertet werden und betrug 7,13 $\pm 8,12$ Tage. Auch hierbei zeigt sich, dass mit zunehmender

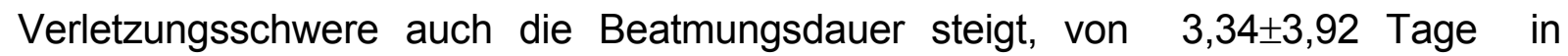

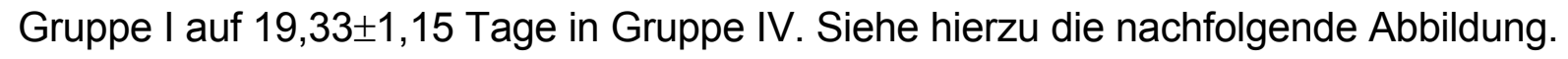

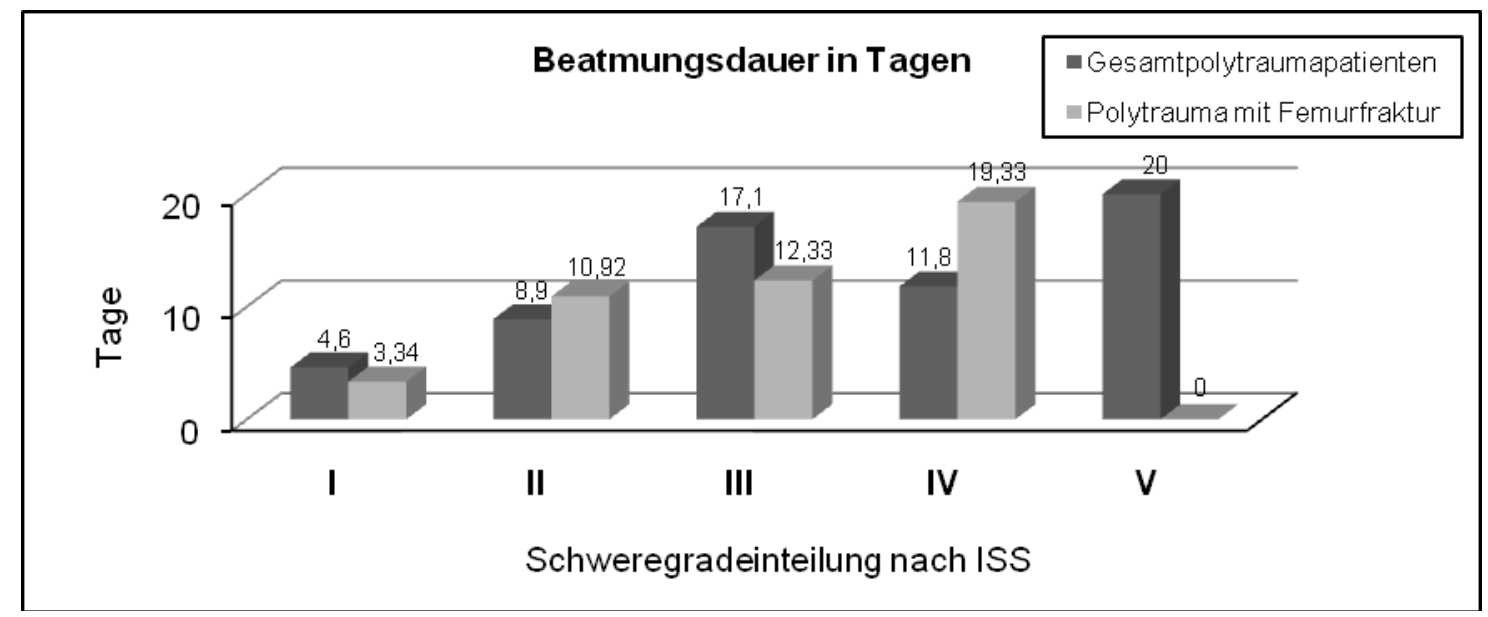

Abbildung 16: Beatmungsdauer nach Schweregradgruppierung. 


\subsubsection{Hämoglobin und Hämatokrit}

Bei jedem Patienten, der im Schockraum aufgenommen wird, wird bei Aufnahme eine Routinelaborkontrolle mit verschiedenen Parametern durchgeführt. Hierzu gehört auch die Bestimmung des Hämoglobins sowie Hämatokritwertes.

Bei der Auswertung der Polytrauma-Datenbank konnten die Hämoglobinwerte bei Aufnahme von 341 Patienten ausgewertet werden. Der durchschnittliche Hb-Wert lag bei 10,2 $\pm 3,01 \mathrm{~g} / \mathrm{dl}$. Bei den überlebenden Patienten lag der durchschnittliche Hb-Wert bei Aufnahme mit 10,6 $\pm 2,75 \mathrm{~g} / \mathrm{dl}$ deutlich über dem Durchschnittswert der verstorbenen Patienten mit $8,3 \pm 3,45 \mathrm{~g} / \mathrm{dl}$.

Bei $85(93,4 \%)$ der 91 Patienten mit Femurfraktur konnte ebenfalls der Hämoglobinwert bei Aufnahme im Schockraum ausgewertet werden. Der durchschnittliche $\mathrm{Hb}-$ Wert lag bei 9,34 $\pm 2,64 \mathrm{~g} / \mathrm{dl}$. Bei den überlebenden Patienten lag der durchschnittliche $\mathrm{Hb}-$ Wert bei Aufnahme mit 9,62 $\pm 2,58 \mathrm{~g} / \mathrm{dl}$ deutlich über dem

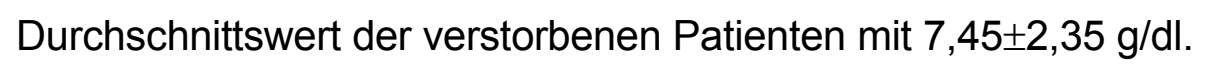

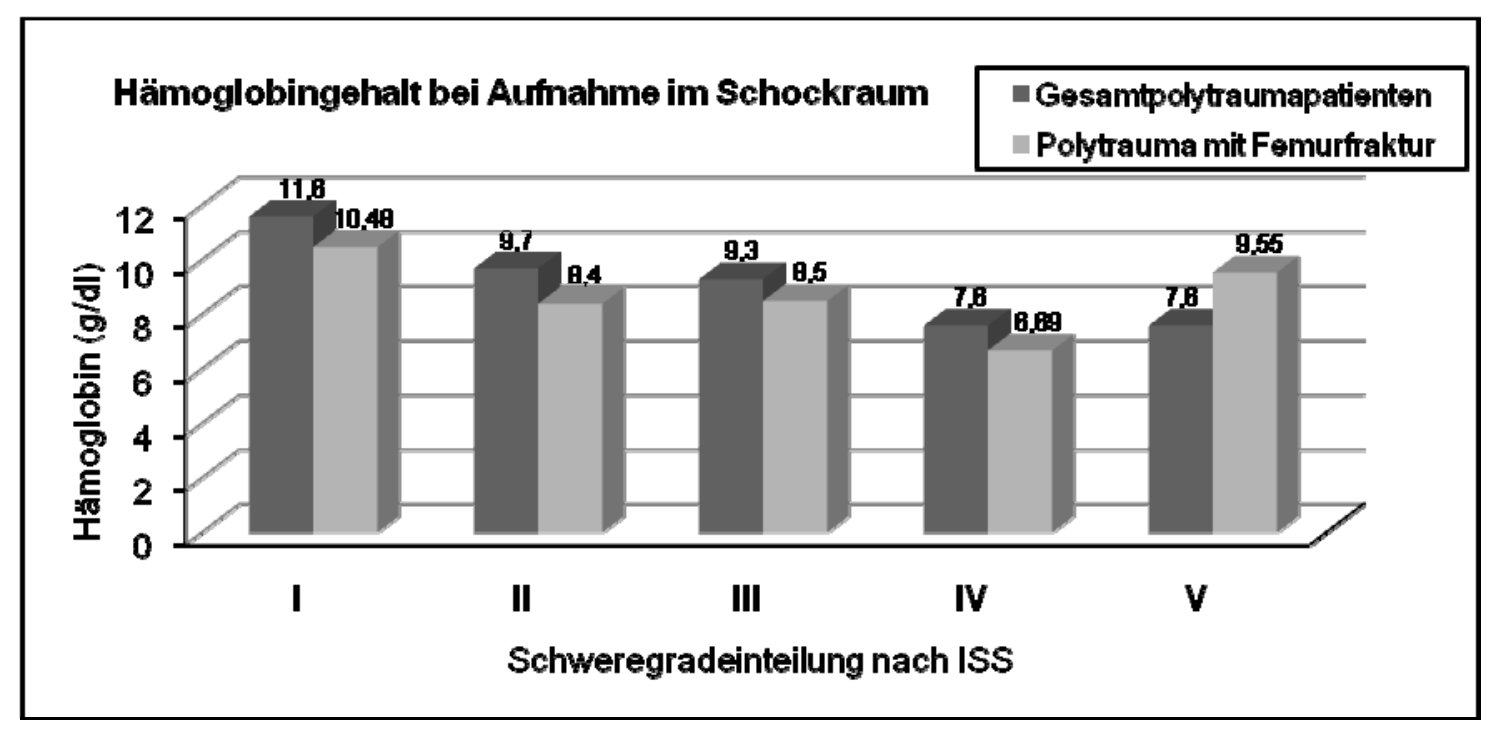

Abbildung 17: Hämoglobin bei Aufnahme.

Bei 301 Patienten aus der Gesamtpolytraumastudie konnte der Hämatokritwert bei Aufnahme ausgewertet werden, der durchschnittliche Hämatokritwert betrug $30,10 \pm 8,70 \%$. Auch hier zeigt sich der Unterschied zwischen den überlebenden Patienten mit einem Durchschnittswert von $31,40 \pm 7,85 \%$ und den verstorbenen 
Patienten mit 23,60 $\pm 9,80 \%$. Mit zunehmender Schwere der Verletzung sinkt der Hämatokritwert von $33,7 \pm 7,25 \%$ in Gruppe I auf $23,5 \pm 6,70 \%$ in Gruppe V.

Bei 82 polytraumatisierten Patienten mit Femurfraktur konnte ein Hämatokritwert zum Aufnahmezeitpunkt erfasst werden, der durchschnittliche Hämatokritwert betrug $27,38 \pm 7,44 \%$. Es zeigte sich ebenfalls ein deutlicher Unterschied zwischen den überlebenden Patienten mit Femurfraktur mit einem Durchschnittswert von $28,25 \pm 7,23 \%$ und den verstorbenen Patienten mit Femurfraktur mit einem Durchschnittswert von 21,76 $\pm 6,50 \%$. Mit zunehmender Schwere der Verletzung sinkt auch hier der Hämatokritwert, von $31,01 \pm 6,11 \%$ in Gruppe I auf $19,42 \pm 7,64 \%$ in Gruppe IV, Gruppe V zeigt einen ansteigenden Hämatokritwert, dabei ist zu beachten, dass diese Gruppe nur 2 Patienten enthält.

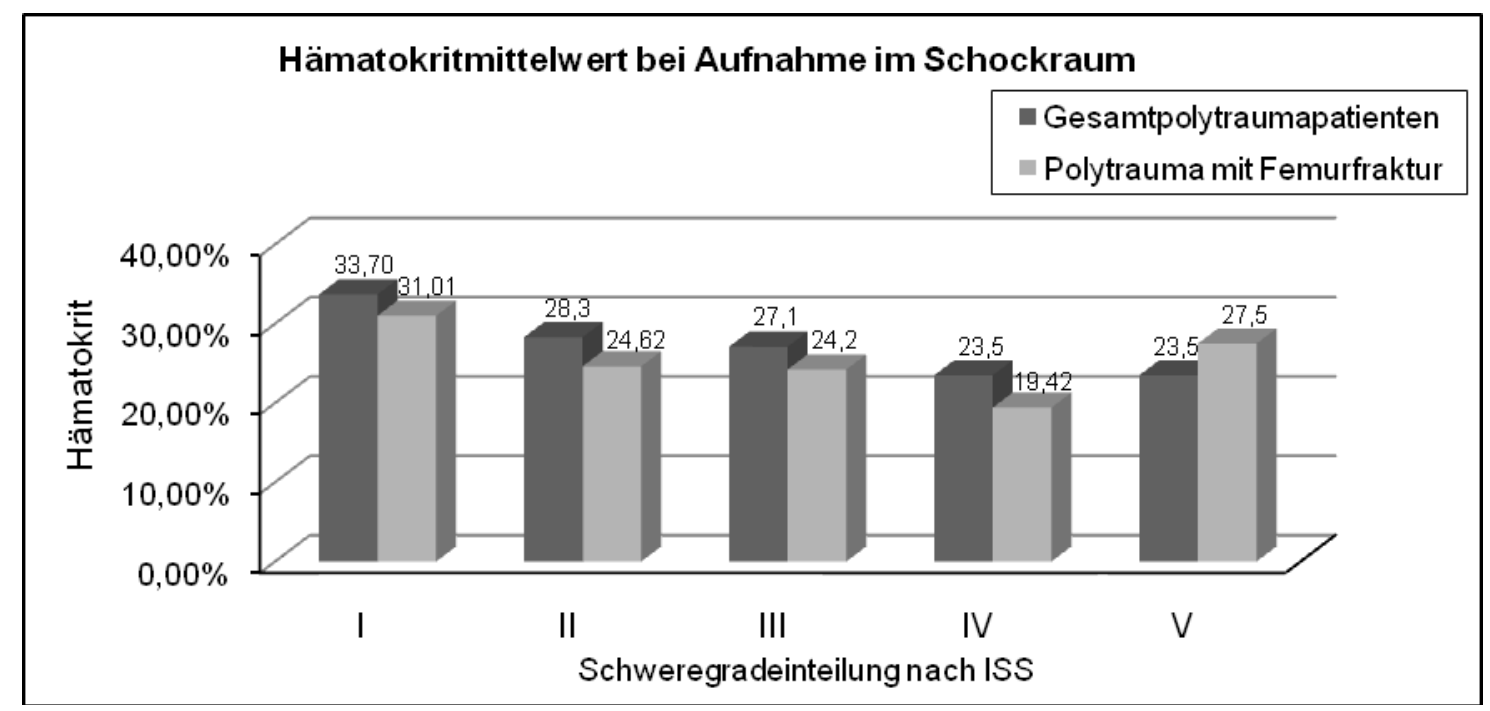

Abbildung 18: Hämatokrit-Mittelwert nach Schweregradgruppierung.

\subsubsection{Zielkrankenhaus}

Bei 567 Patienten (82,9\%) von den 684 insgesamt behandelten Polytraumapatienten konnte das primäre Zielkrankenhaus dokumentiert werden. 378 (66,7\%) der Verletzten wurden primär in die Universitätsmedizin Göttingen aufgenommen, 189 $(33,3 \%)$ Patienten wurden erst sekundär aus einem anderen primären Zielkrankenhaus nach Göttingen verlegt. Die Verteilung auf die einzelnen Schweregradgruppen ist aus der folgenden Tabelle zu entnehmen. Hierbei zeigt sich, 
dass mit zunehmender Verletzungsschwere nach ISS der prozentuale Anteil, der primär in der Universitätsmedizin Göttingen versorgt wird, stetig ansteigt.

Tabelle 7: Primär- und Sekundärversorgung der Gesamtpolytraumapatienten.

\begin{tabular}{|l|c|c|c|c|c|}
\hline & $\begin{array}{l}\text { Gesamt- } \\
\text { anzahl (N=) }\end{array}$ & \multicolumn{2}{|c|}{$\begin{array}{c}\text { Primärversorgung } \\
\text { Uni Göttingen } \\
\text { Prozent- } \\
\text { angabe (\%) }\end{array}$} & \multicolumn{2}{c|}{ Anzahl $\begin{array}{c}\text { Prozent- } \\
\text { angabe (\%) }\end{array}$} \\
\hline $\begin{array}{l}\text { Gesamtpoly- } \\
\text { traumapatienten }\end{array}$ & 567 & 378 & 66,7 & 189 & 33,3 \\
\hline ISS 16-25 & 288 & 181 & 62,8 & 107 & 37,2 \\
\hline ISS 26-35 & 135 & 94 & 69,6 & 41 & 30,4 \\
\hline ISS 36-45 & 79 & 55 & 69,6 & 24 & 30,4 \\
\hline ISS 46-55 & 24 & 18 & 75,0 & 6 & 25,0 \\
\hline ISS $\geq 55$ & 12 & 9 & 75,0 & 3 & 25,0 \\
\hline Überlebende & 464 & 300 & 64,7 & 164 & 35,3 \\
\hline Verstorbene & 103 & 78 & 75,7 & 25 & 24,3 \\
\hline
\end{tabular}

Bei 89 Patienten $(97,8 \%)$ von den 91 Polytraumatisierten mit Femurfraktur konnte das primäre Zielkrankenhaus dokumentiert werden. 70 Patienten $(78,65 \%)$ wurden primär in die Universitätsmedizin Göttingen aufgenommen, 19 (21,35\%) Patienten wurden erst sekundär aus einem anderen primären Zielkrankenhaus nach Göttingen verlegt. Hierbei zeigt sich nicht, wie bei dem Gesamtkollektiv der Polytraumapatienten, dass mit zunehmender Verletzungsschwere nach ISS der prozentuale Anteil, der primär in der Universitätsmedizin Göttingen versorgt wird, stetig ansteigt. Lediglich die besonders schwer verletzten Patienten mit einem ISS >55 wurden zu 100\% primär nach Göttingen gebracht.

Tabelle 8 : Primär- und Sekundärversorgung der Polytraumapatienten mit Femurfraktur.

\begin{tabular}{|c|c|c|c|c|c|}
\hline & $\begin{array}{l}\text { Gesamt- } \\
\text { anzahl } \\
(\mathrm{N}=)\end{array}$ & $\begin{array}{l}\text { Prim } \\
\text { Un } \\
\text { Anza }\end{array}$ & $\begin{array}{l}\text { rsorgung } \\
\text { ttingen } \\
\text { Prozent- } \\
\text { angabe }\end{array}$ & Anzahl & $\begin{array}{l}\text { erlegung } \\
\text { Prozent- } \\
\text { angabe }\end{array}$ \\
\hline $\begin{array}{l}\text { Polytraumapatienten } \\
\text { mit Femurfraktur }\end{array}$ & 89 & 70 & $78,65 \%$ & 19 & $21,35 \%$ \\
\hline ISS 16-25 & 42 & 35 & $83,33 \%$ & 7 & $16,67 \%$ \\
\hline ISS 26-35 & 31 & 24 & $77,42 \%$ & 7 & $22,58 \%$ \\
\hline ISS 36-45 & 9 & 6 & $66,66 \%$ & 3 & $33,34 \%$ \\
\hline ISS 46-55 & 5 & 3 & $60,00 \%$ & 2 & $40,00 \%$ \\
\hline ISS $\geq 55$ & 2 & 2 & $100,00 \%$ & 0 & $0 \%$ \\
\hline Überlebende & 78 & 65 & $83,33 \%$ & 13 & $16,67 \%$ \\
\hline Verstorbene & 11 & 9 & $81,82 \%$ & 2 & $18,18 \%$ \\
\hline
\end{tabular}




\subsubsection{Unfallursache}

Mit 55\% tragen die PKW-Unfälle den Hauptanteil an den Unfallursachen für die polytraumatisierten Patienten. Im Vergleich hierzu war die Unfallursache in $67 \%$ der Patienten mit Femurfraktur der PKW-Unfall. Mit 15\% lag der Anteil, der mit dem Motorrad verunfallten Patienten, welche sich eine Femurfraktur zugezogen hatten, im Vergleich zur Gesamtpolytraumastudie etwas höher. Weitere Unfallursachen im Vergleich zeigen die folgenden Abbildungen.
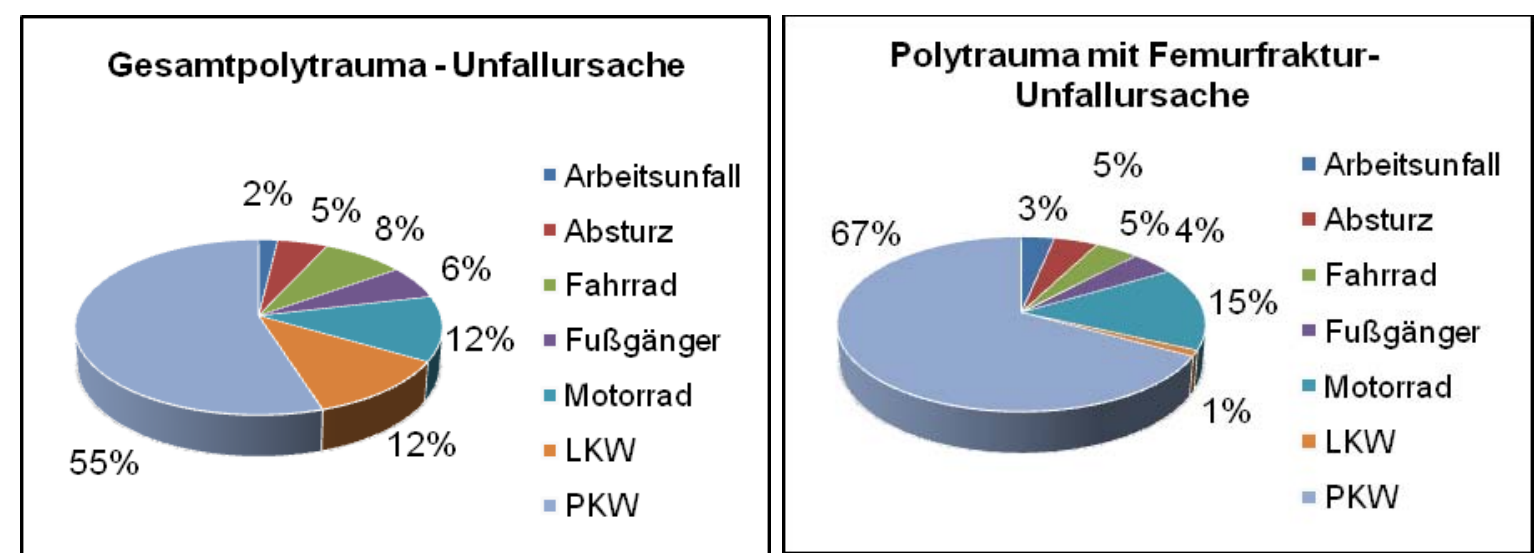

Abbildung 19/20: Unfallursache.

3.4. präklinische und klinische Daten der Polytraumapatienten mit Femurfraktur im Gruppenvergleich

Entsprechend den Erläuterungen im Methodenteil erfolgte die Einteilung der polytraumatisierten Patienten mit Femurfraktur in 2 Gruppen je nach Operationszeitpunkt der Primärversorgung der Femurfraktur. Im nachfolgenden Ergebnisteil sind die gewonnenen Daten im Vergleich aufgeführt.

\subsubsection{Geschlechts- und Altersverteilung}

Wie im Gesamtkollektiv der an der Universitätsmedizin Göttingen im Untersuchungszeitraum behandelten Polytraumapatienten ist auch in diesem Teil der Untersuchung erkennbar, dass deutlich mehr männliche Patienten als weibliche 
betroffen sind. Im Vergleich der beiden Gruppen zeigte sich kein signifikanter Unterschied $(p=0,097)$ im Bezug auf die Geschlechtsverteilung.

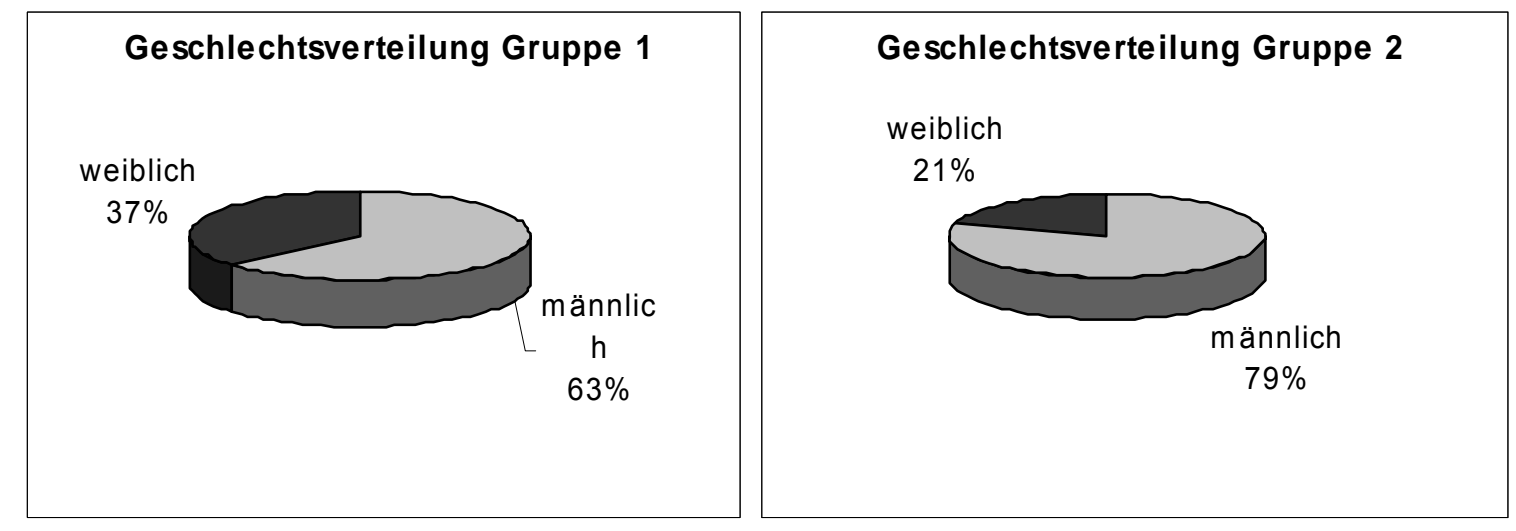

Abbildung 21/22: Geschlechtsverteilung in Gruppe 1 und 2.

Bei der Altersverteilung zeigt sich, dass die am häufigsten betroffene Altersgruppe , die zwischen dem 21.-30. Lebensjahr sowohl in Gruppe 1 wie auch in Gruppe 2 darstellt. Ein signifikanter Unterschied zwischen den beiden Gruppen zeigte sich nicht $(p=0,760)$. In der folgenden Abbildung ist die Altersverteilung der beiden Gruppen aufgezeigt.

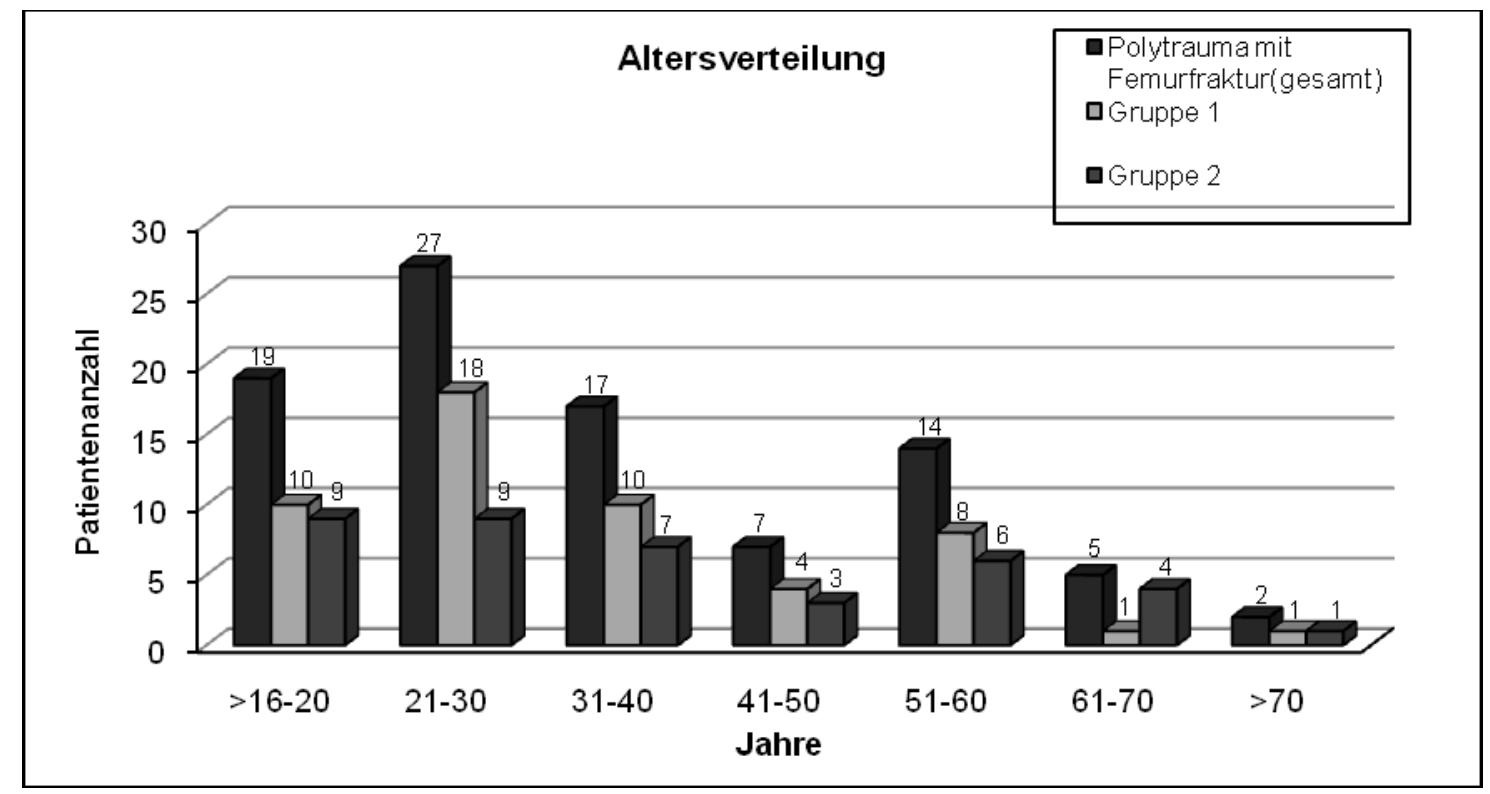

Abbildung 23: Altersverteilung. 


\subsubsection{Jahresverteilung}

Ein signifikanter Unterschied zwischen den beiden Gruppen zeigte sich bei der Jahresverteilung mit $p=0,032$. Wie auch in der folgenden Abbildung erkennbar ist, gibt es einen Gipfel der Patientenanzahl in der Gruppe 1, in der frühzeitigen Frakturversorgung innerhalb der ersten 6 Stunden im Jahre 1995, während in der Gruppe 2 sich die höchste Patientenanzahl im Jahre 1998 findet.

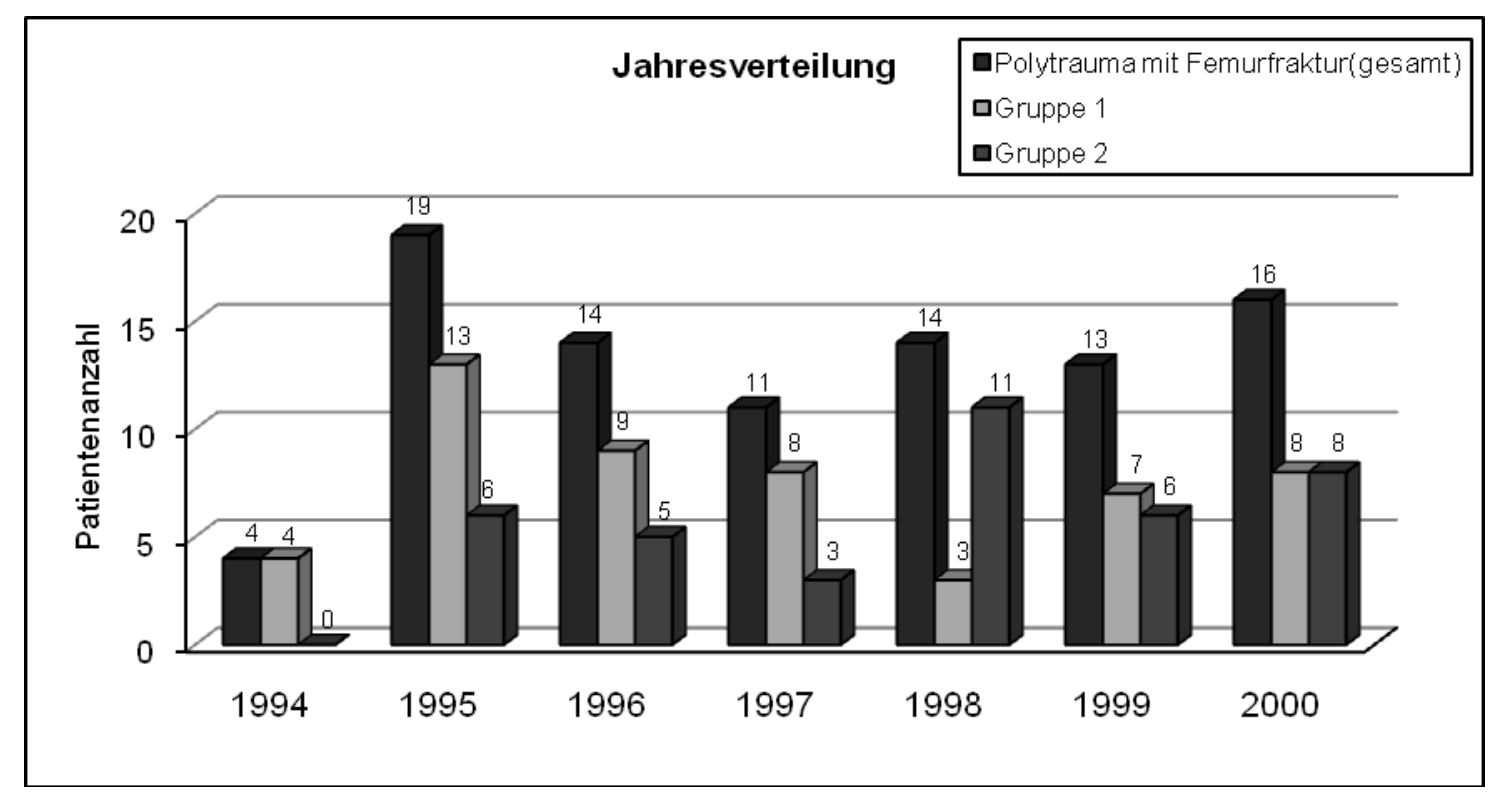

Abbildung 24: Jahresverteilung in Gruppe 1 und 2.

\subsubsection{Schweregradverteilung nach ISS}

In Übereinstimmung mit den bereits vorliegenden Arbeiten über das Gesamtkollektiv der Polytraumapatienten ist auch bei der Untersuchung der Femurfrakturen der größte prozentuale Anteil an Patienten in beiden Gruppen ein Schweregrad nach ISS von 16-25. Ein signifikanter Unterschied zwischen beiden Gruppen war nicht nachweisbar $(p=0,417)$. Der ISS-Mittelwert der Gruppe 1 betrug 29,29 $\pm 11,72$ und für Gruppe 2 $27,18 \pm 9,62$. 


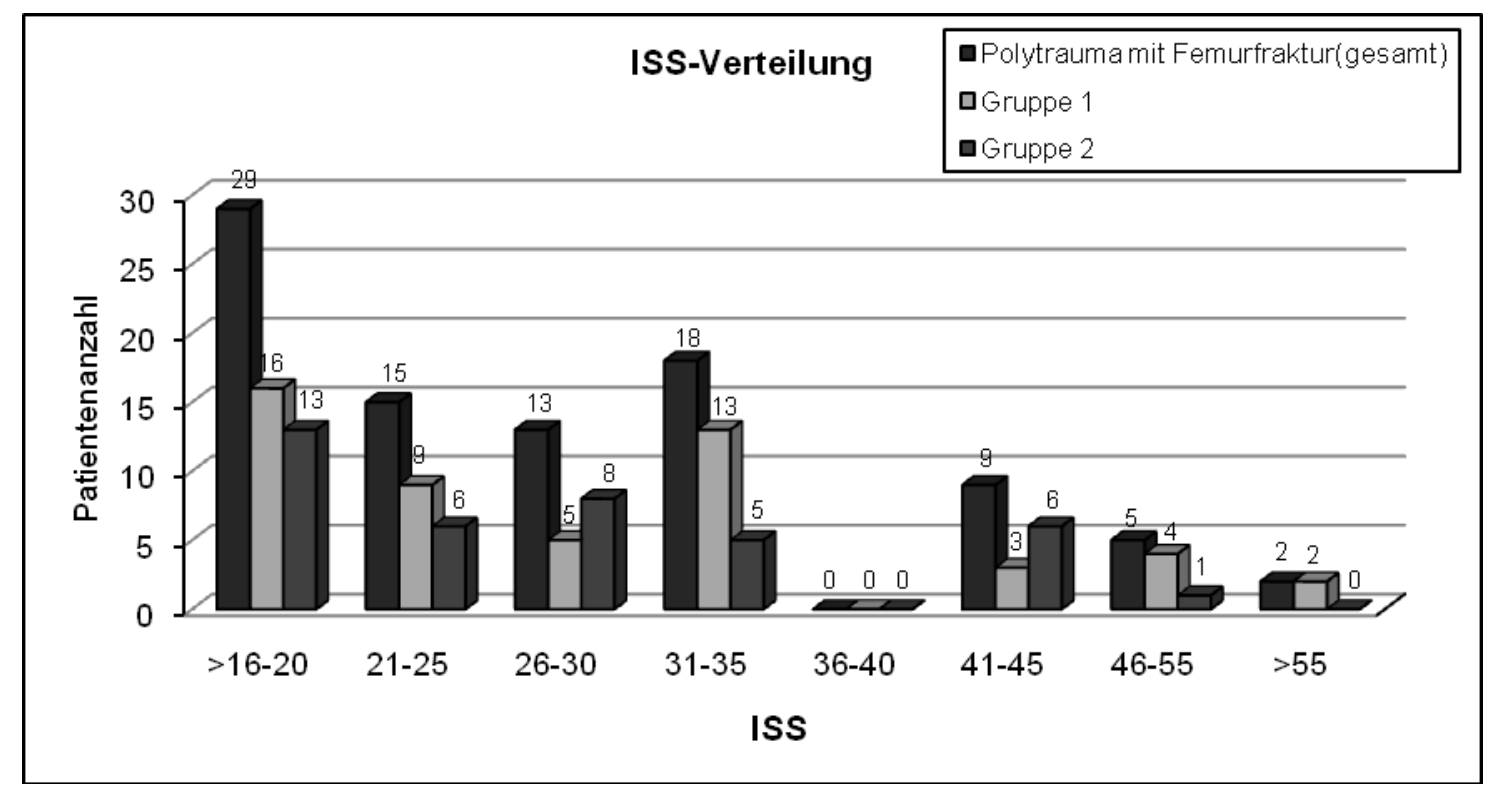

Abbildung 25: Schweregrad nach ISS in Gruppe 1 und 2.

\subsubsection{Zielkrankenhaus}

Ein signifikanter Unterschied zwischen den beiden zu untersuchenden Gruppen zeigte sich bei der Auswahl des Primärkrankenhauses bzw. einer sekundären Verlegung mit $p=0,002$. In der Gruppe 1 beträgt der prozentuale Anteil der Patienten, welche primär in die Universitätsmedizin Göttingen gebracht wurden 90,38\%, im Vergleich hierzu beträgt der Anteil bei Gruppe 2 nur 62,16\%. Nur 9,62\% der Patienten der Gruppe 1 wurden erst sekundär nach Göttingen verlegt, in Gruppe 2 waren es 37,84\% der Patienten.

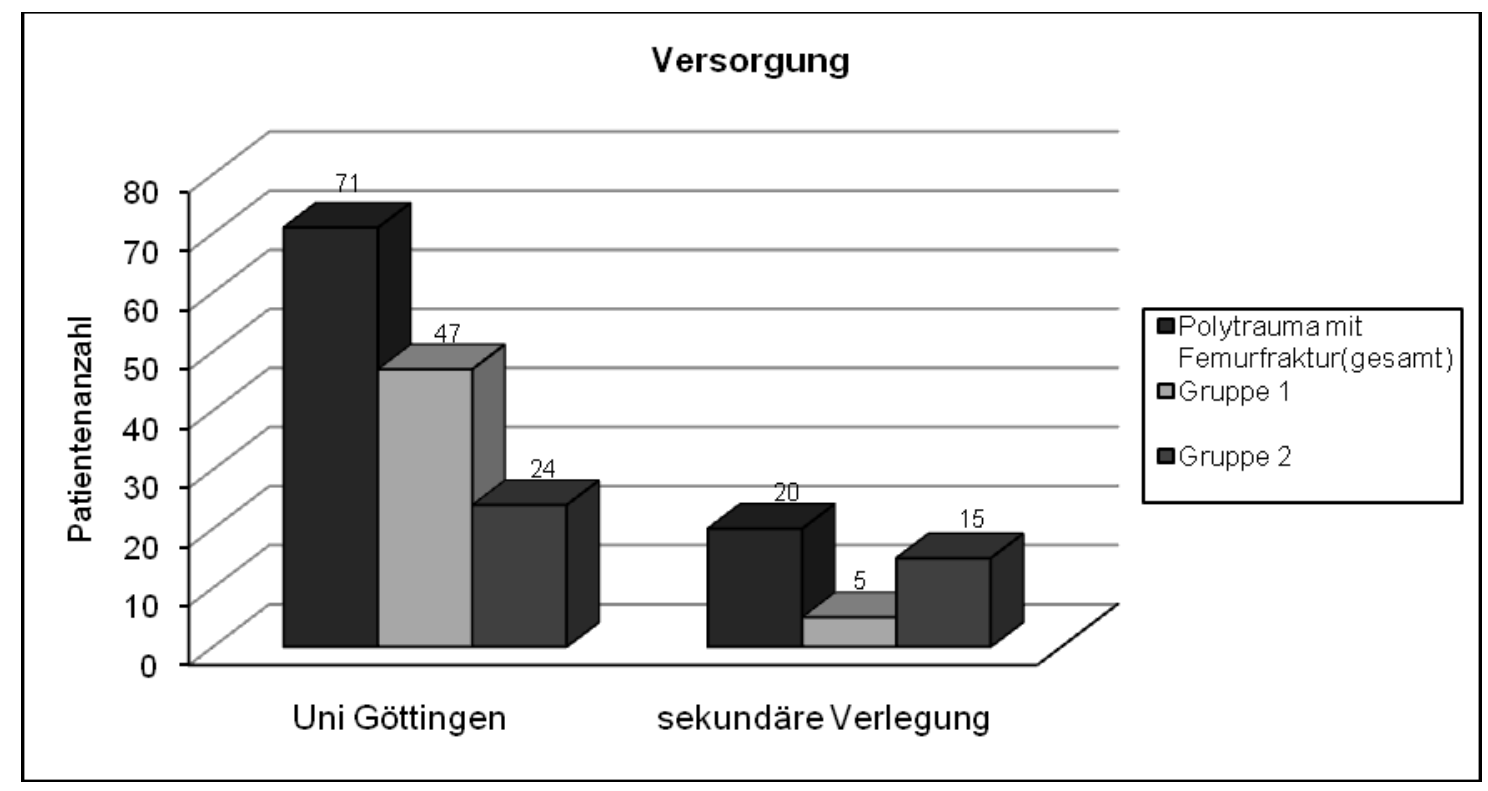

Abbildung 26: Primärversorgung bzw. Sekundärverlegung in Gruppe 1 und 2. 


\subsubsection{Unfallursache}

Bei dem Vergleich der Unfallursachen für die beiden Gruppen zeigte sich mit $p=0,018$ ein signifikanter Unterschied dahin gehend, dass in Gruppe 1 deutlich mehr Motorradunfälle als in Gruppe 2 zu verzeichnen waren, und zum anderen in Gruppe 2 deutlich mehr Absturzunfälle im Vergleich zu Gruppe 1. Die Verteilung der Patienten ist auch der nachfolgenden Abbildung zu entnehmen.

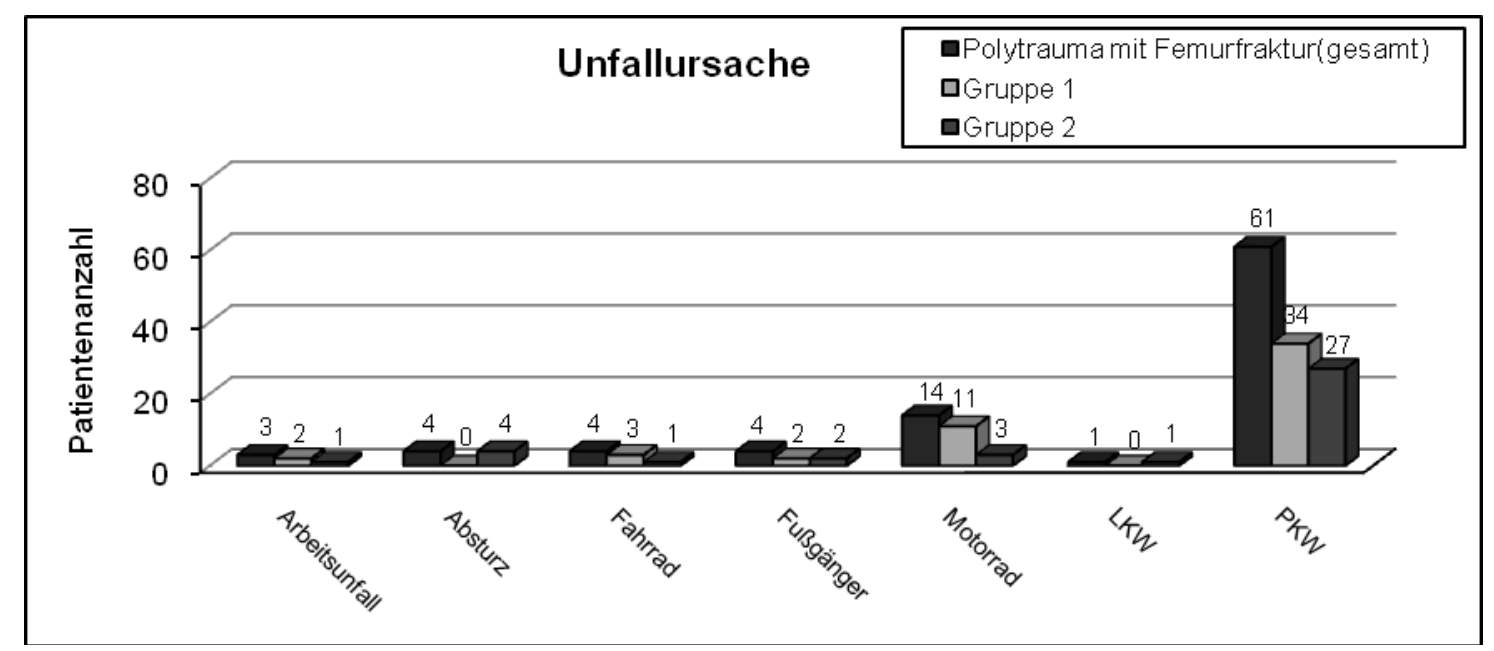

Abbildung 27: Unfallursachen in Gruppe 1 und 2.

\subsubsection{Verletzungsmuster}

Beim Vergleich der Verletzungsmuster beider Gruppen zeigte sich kein signifikanter Unterschied $(p=0,164)$, in beiden Gruppen ist der größte Anteil der betroffenen Regionen Kopf- und Thoraxverletzungen, wie in der nachfolgenden Abbildung erkennbar ist.

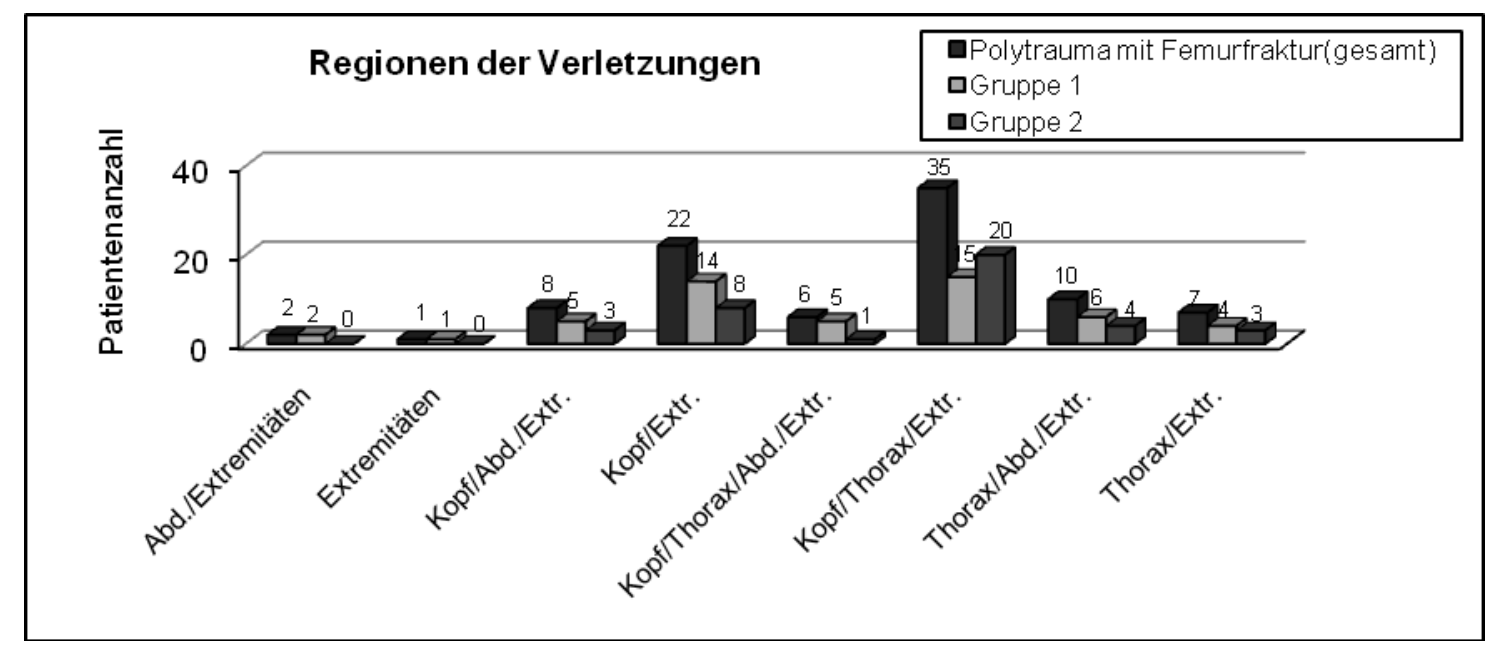

Abbildung 28: Verletzungsmuster. 


\subsubsection{Seitenverteilung der Femurfrakturen und Grad der offenen Frakturen}

In beiden Gruppen zeigte sich, dass in mehr als 50\% der Fälle die linke Seite betroffen war. In Gruppe 1 war die linke Seite in 53,45\% betroffen, in Gruppe 2 in 60,47\%. Die rechte Seite war deutlich weniger betroffen, in Gruppe $125,86 \%$ und in Gruppe 2 20,93\%. Beidseitige Frakturen fanden sich in 10,34\% der Gruppe 1 und in 6,89\% der Gruppe 2. Ein signifikanter Unterschied zwischen den beiden Gruppen zeigte sich nicht $(p=0,529)$.

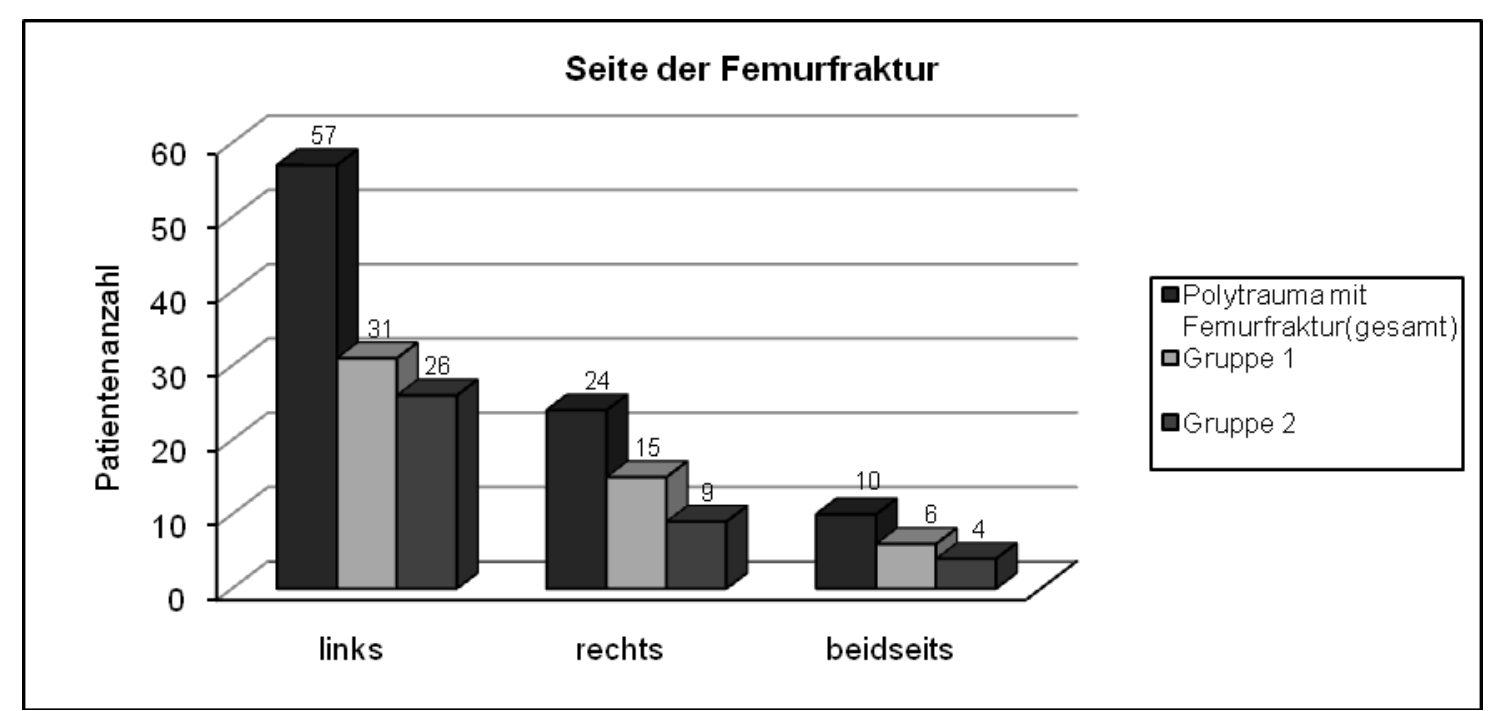

Abbildung 29: Verteilung der betroffenen Seite.

Bei 14 (24,14\%) Femurfrakturen der Gruppe 1 handelte es sich um offene Frakturen, bei $12(27,91 \%)$ der Gruppe 2. Bei der Differenzierung des Ausmaßes der offenen Frakturen zeigte sich zwischen den Gruppen mit $p=0,846$ kein signifikanter Unterschied.

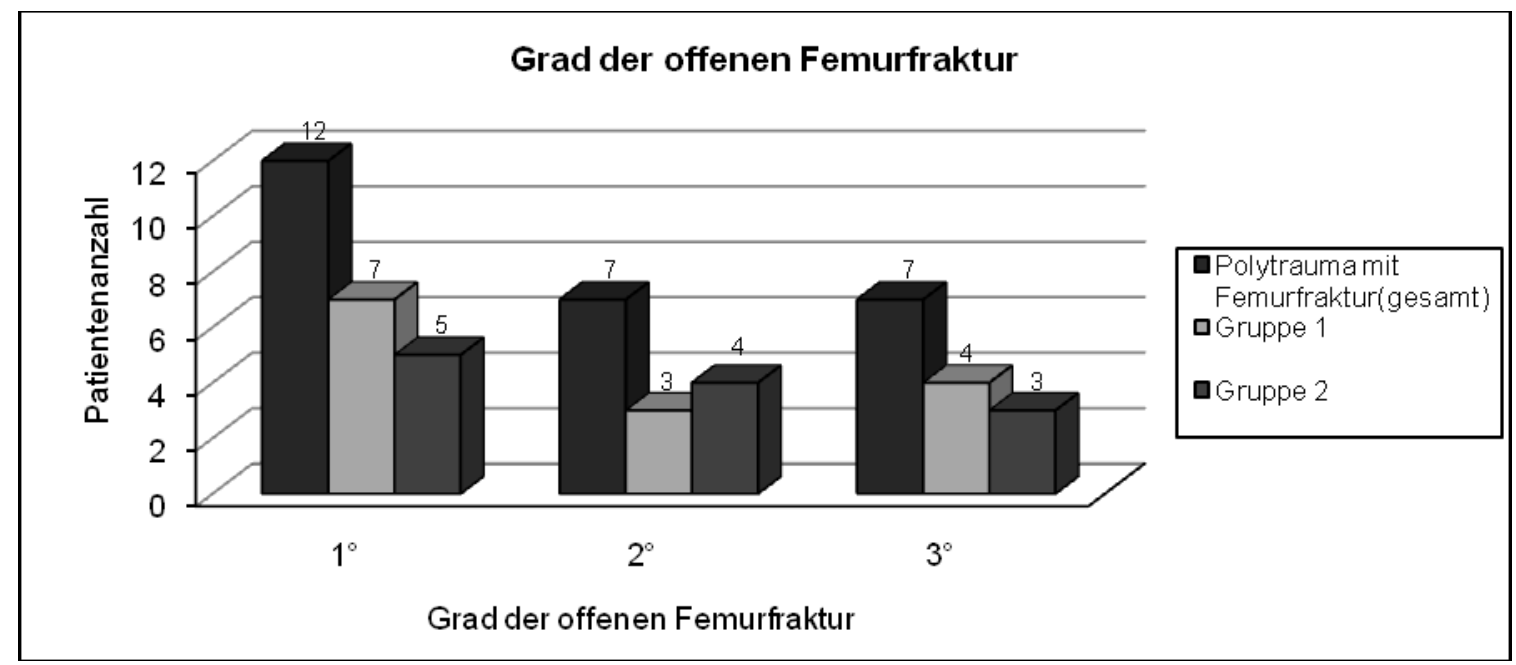

Abbildung 30: Grad der offenen Femurfrakturen. 


\subsubsection{AO-Klassifikation}

Bei 27,59\% der Fälle der Gruppe 1 konnte keine AO-Klassifikation erfolgen, da keine Röntgenaufnahmen zum Untersuchungszeitpunkt mehr im Archiv vorlagen, in Gruppe 2 konnte bei 9,30\% keine Klassifikation erfolgen. Beim Vergleich der nach AOklassifizierten Femurfrakturen der beiden zu untersuchenden Gruppen zeigte sich kein signifikanter Unterschied $(p=0,628)$. In der nachfolgenden Abbildung ist erkennbar, dass den Hauptanteil in beiden Gruppen die Femurschaftfrakturen ausmachen, mit 46,55\% in Gruppe 1 und mit 62,79\% in Gruppe 2.

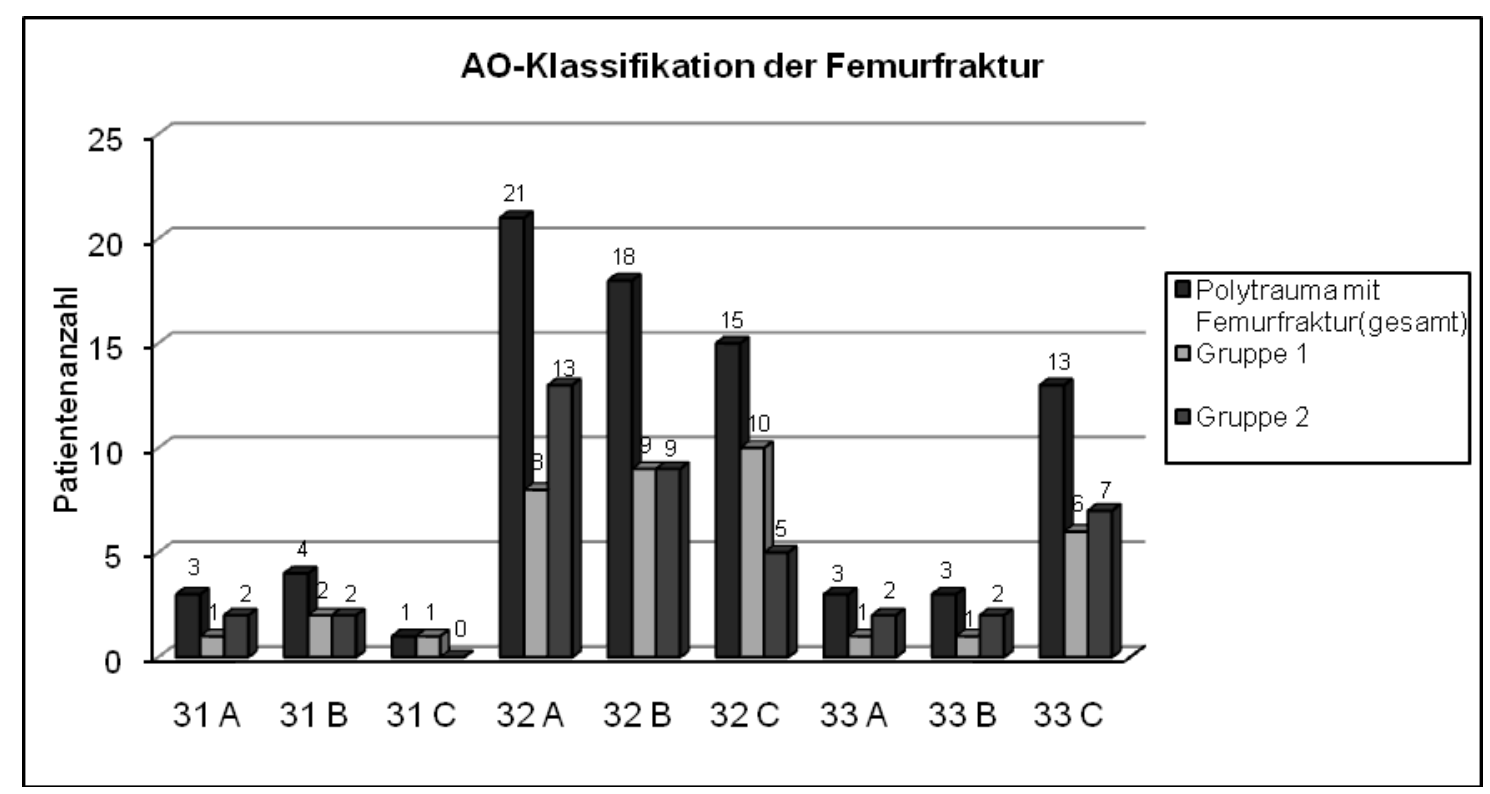

Abbildung 31: Klassifizierung der Femurfrakturen nach AO.

\subsubsection{Primär- und Sekundär-Operation zur Frakturversorgung am Femur}

Bei 54 der Femurfrakturen (93,10\%) der Gruppe 1 konnte die primäre Operation zur Frakturversorgung aus den vorliegenden Akten ausgewertet werden, in Gruppe 2 waren es 43 Femurfrakturen (100\%). Ein signifikanter Unterschied zwischen den beiden Gruppen konnte bei der Primär-Operation nicht gezeigt werden $(p=0,069)$.

37,93\% der Femurfrakturen der Gruppe 1 wurden primär mit einem Fixateur extern versorgt, im Vergleich hierzu wurden jedoch nur 9,30\% der Gruppe 2 mit einem Fixateur extern versorgt. Vergleicht man die Patienten beider Gruppen, die mit einem Fixateur extern versorgt wurden mit denen, die einen Marknagel erhielten, so zeigen sich signifikante Unterschiede hinsichtlich des ISS $(p=0,0005)$, sowie der 
Intensivaufenthaltsdauer $(p=0,0009)$ und Beatmungsdauer $(p=0,00046)$. Der Mittelwert des ISS der Patienten mit Fixateur extern betrug 36,15 $\pm 12,05$, die Intensivaufenthaltsdauer betrug 28,63 $\pm 21,13$ Tage und die Beatmungsdauer 10,8 $\pm 11,17$ Tage. Im Vergleich hierzu lag der ISS der Patienten, die mit einem Marknagel versorgt wurden, bei $21,95 \pm 4,68$, die Intensivaufenthaltsdauer betrug

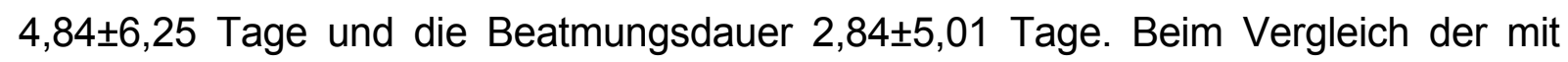
Fixateur extern versorgten Patienten mit denen, die eine Plattenosteosynthese am Femur erhielten, zeigen sich ebenfalls signifikante Unterschiede hinsichtlich des ISS $(p=0,019)$ und der Intensivaufenthaltsdauer $(p=0,0042)$. Der ISS-Mittelwert für die plattenosteosynthetisch versorgten Patienten betrug 28,04 $\pm 9,55$, die Intensivaufenthaltsdauer $14,46 \pm 12,30$ Tage.

In der nachfolgenden Abbildung sind die Primär-Operationen am Femur der jeweiligen Gruppen aufgeführt.

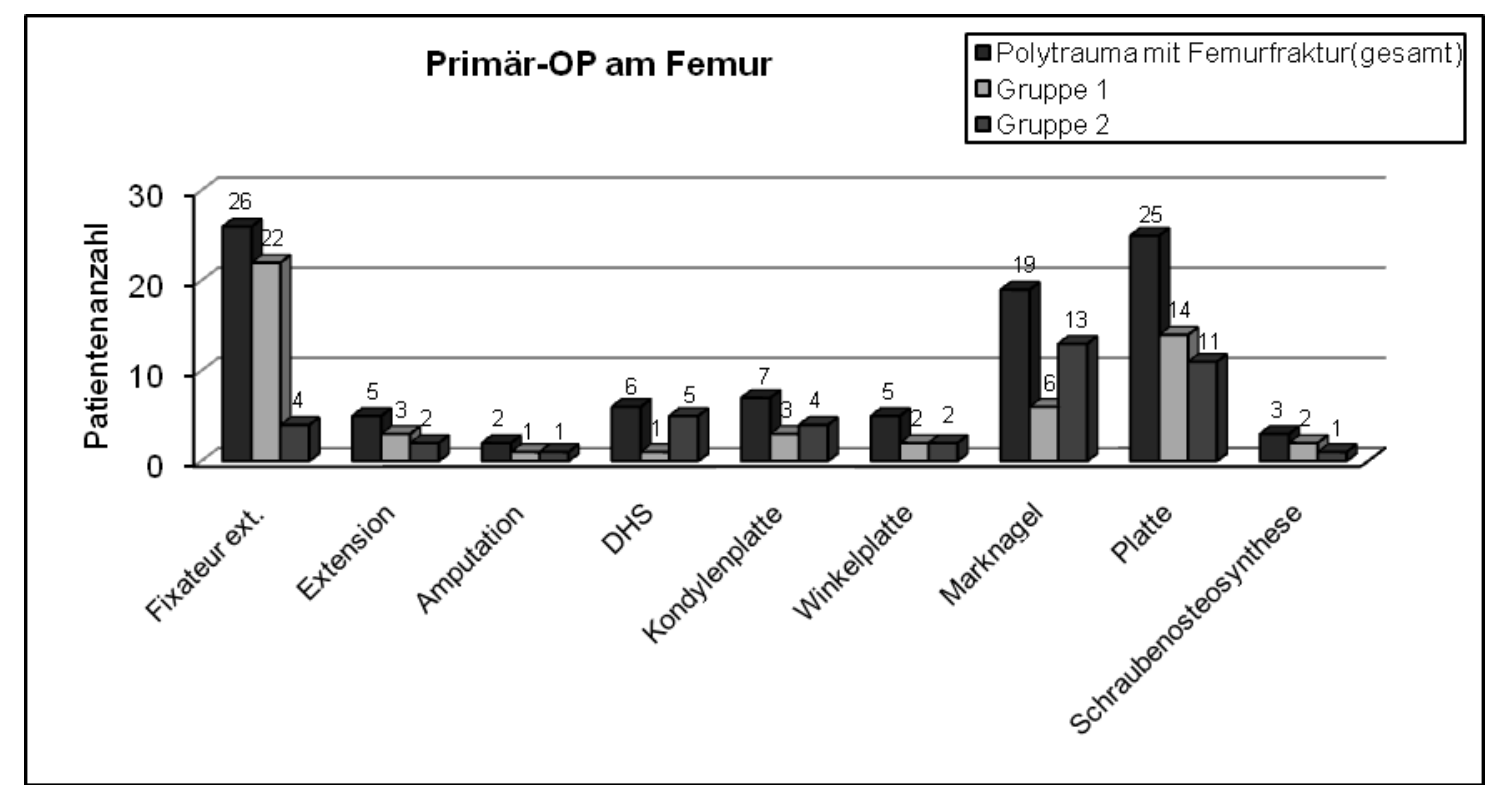

Abbildung 32: Verteilung der Primär-Operation am Femur.

Bei 16 Femurfrakturen (27,59\%) der in die Gruppe 1 eingestuften Femurfakturen $(\mathrm{N}=58)$ wurde eine Sekundär-Operation am Femur zur Frakturversorgung durchgeführt, 9 Patienten waren im weiteren Verlauf bereits verstorben. Im Vergleich hierzu wurden 5 Patienten mit Femurfrakturen (11,63\%) der Gruppe 2 (N=43) nochmals sekundäroperativ versorgt. Einen signifikanten Unterschied in der 
sekundäroperativen Versorgung war zwischen den zu untersuchenden Gruppen nicht nachweisbar $(p=0,942)$.

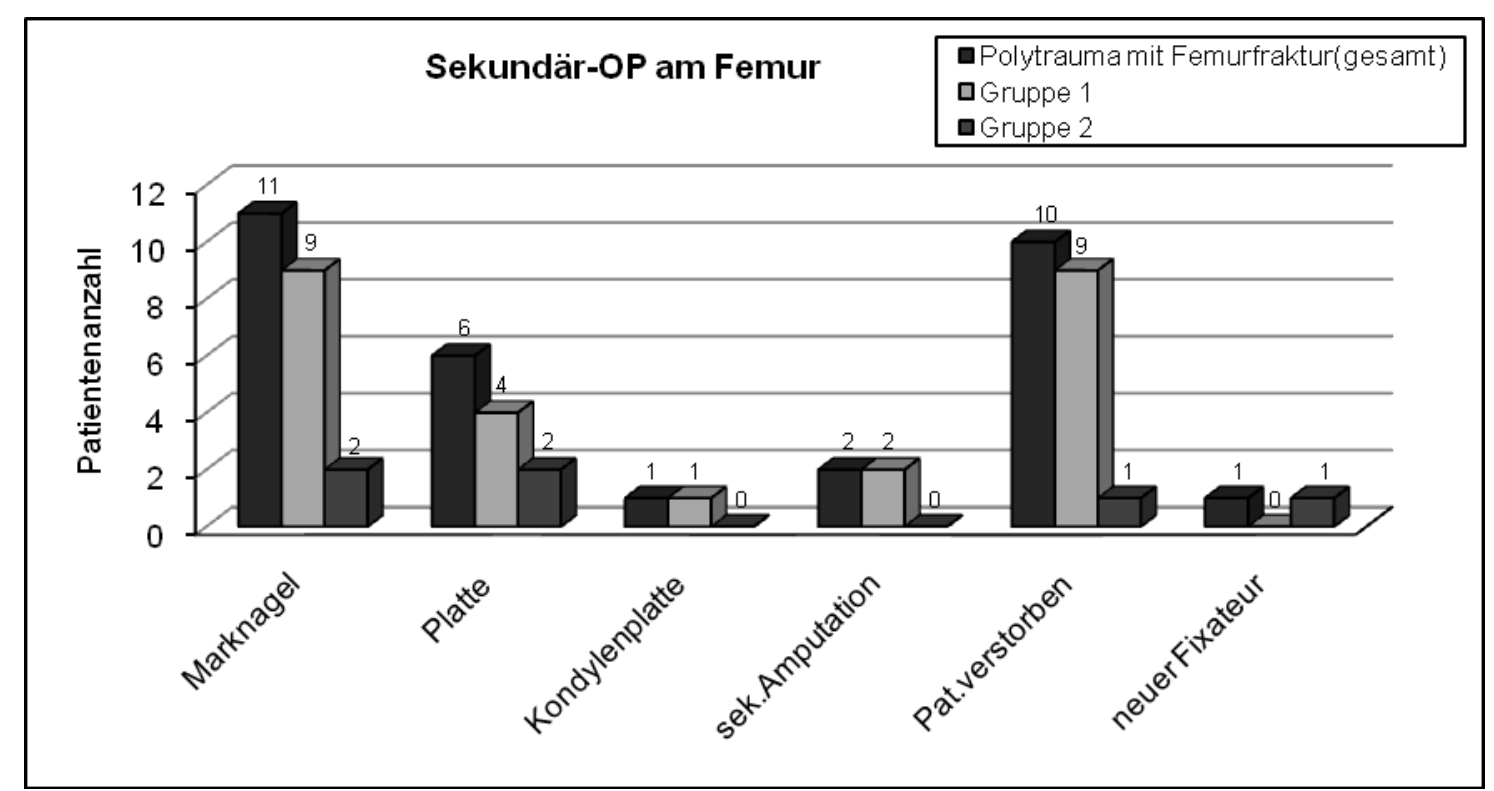

Abbildung 33: Verteilung der Sekundär-Operation am Femur.

3.4.10. Intensiv- und Beatmungsdauer

Bei 42 Patienten (80,77\%) der Gruppe 1 konnte die Intensivaufenthaltsdauer erfasst und ausgewertet werden, in Gruppe 2 erfolgte dies bei 38 Patienten (97,44\%). Der Mittelwert der Intensivaufenthaltsdauer in Gruppe 1 betrug 14,17 \pm 15,40 Tage, in Gruppe 2 betrug der Mittelwert für den Intensivaufenthalt 16,60 \pm 19,52 Tage. In der nachfolgenden Abbildung ist die Intensivaufenthaltsdauer nochmals aufgeführt. 


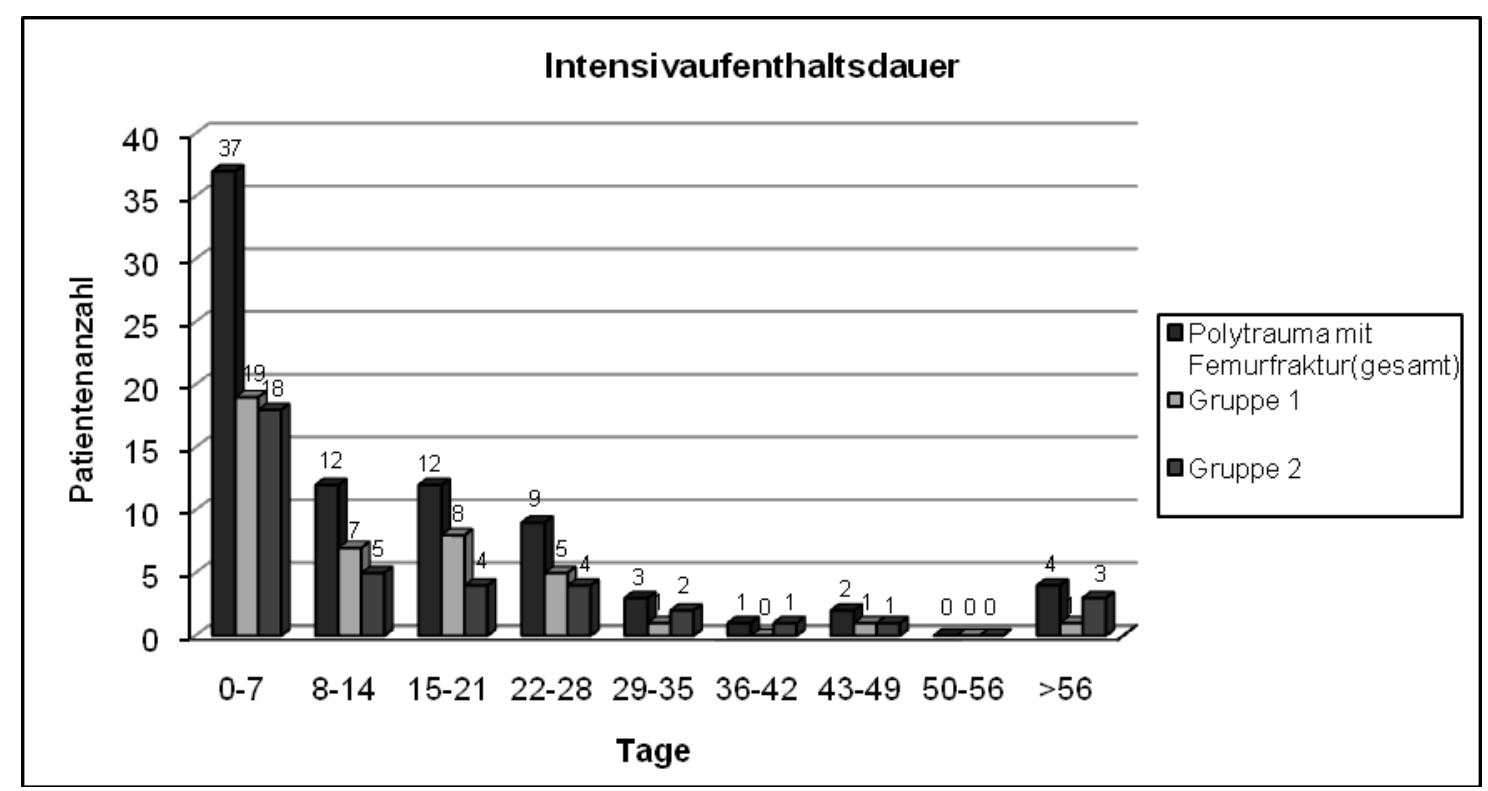

Abbildung 34: Intensivaufenthaltsdauer.

Die Beatmungsdauer konnte bei 42 Patienten (80,77\%) der Gruppe 1 und bei 37 $(94,87 \%)$ der Gruppe 2 erfasst und berechnet werden. Der Mittelwert der Beatmungsdauer der Gruppe 1 lag bei 7,24 Tagen $\pm 8,19$, in Gruppe 2 bei 7,0 Tagen $\pm 8,17$. In der nachfolgenden Abbildung sind die Beatmungsdauer der jeweiligen Gruppen aufgeführt. Ein signifikanter Unterschied zwischen den zu untersuchenden Gruppen fand sich nicht $(p=0,478)$.

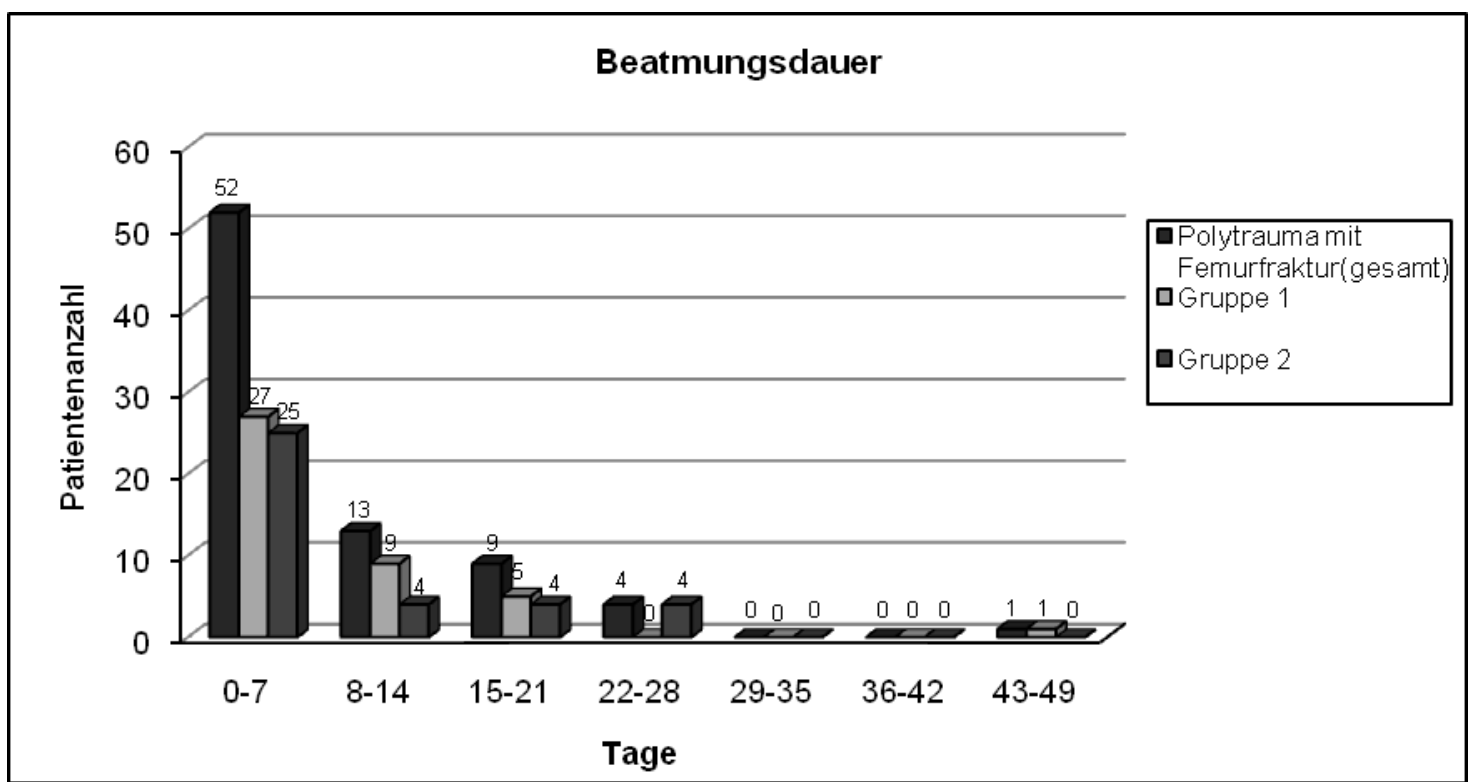

Abbildung 35: Beatmungsdauer.

3.4.11. Hämoglobin und Hämatokrit 
Zur Auswertung des Hämoglobinwertes bei Aufnahme im Schockraum konnten in Gruppe 1 bei 50 Patienten (96,15\%) die Daten erhoben werden, in Gruppe 2 bei 35 Patienten (89,74\%). Der Mittelwert des Hämoglobins bei Aufnahme im Schockraum in Gruppe 1 betrug 9,17g/dl $\pm 3,01$, in Gruppe 2 9,5g/dl $\pm 2,02$.

Tabelle 9: Hämoglobinwert bei Klinikaufnahme nach Schweregradgruppierung unterteilt für Gruppe 1.

\begin{tabular}{|l|l|l|l|l|l|}
\hline & $\mathrm{N}$ & $\begin{array}{l}\text { Mittelwert } \\
(\mathrm{g} / \mathrm{dl})\end{array}$ & $\begin{array}{l}\text { Minimum } \\
(\mathrm{g} / \mathrm{dl})\end{array}$ & $\begin{array}{l}\text { Maximum } \\
(\mathrm{g} / \mathrm{dl})\end{array}$ & $\begin{array}{l}\text { Standard- } \\
\text { abweichung }\end{array}$ \\
\hline ISS 16-25 & 24 & 10,53 & 3,90 & 14,60 & 2,58 \\
\hline ISS 26-35 & 17 & 8,08 & 3,60 & 12,90 & 2,71 \\
\hline ISS 36-45 & 3 & 7,90 & 2,80 & 10,50 & 4,42 \\
\hline ISS 46-55 & 4 & 6,03 & 3,43 & 8,40 & 2,63 \\
\hline ISS $\geq 55$ & 2 & 9,55 & 9,10 & 10,00 & 0,64 \\
\hline Überlebende & 40 & 9,59 & 3,43 & 14,60 & 3,01 \\
\hline Verstorbene & 10 & 7,50 & 2,80 & 10,60 & 2,47 \\
\hline
\end{tabular}

Tabelle 10: Hämoglobinwert bei Klinikaufnahme nach Schweregradgruppierung unterteilt für Gruppe 2.

\begin{tabular}{|l|l|l|l|l|l|}
\hline & $\mathrm{N}$ & $\begin{array}{l}\text { Mittelwert } \\
(\mathrm{g} / \mathrm{dl})\end{array}$ & $\begin{array}{l}\text { Minimum } \\
(\mathrm{g} / \mathrm{dl})\end{array}$ & $\begin{array}{l}\text { Maximum } \\
(\mathrm{g} / \mathrm{dl})\end{array}$ & $\begin{array}{l}\text { Standard- } \\
\text { abweichung }\end{array}$ \\
\hline ISS 16-25 & 17 & 10,31 & 6,90 & 12,90 & 2,00 \\
\hline ISS 26-35 & 12 & 8,85 & 6,00 & 13,80 & 2,18 \\
\hline ISS 36-45 & 5 & 8,86 & 7,00 & 9,60 & 1,06 \\
\hline ISS 46-55 & 1 & 9,30 & 9,30 & 9,30 & 0 \\
\hline ISS $\geq 55$ & 0 & 0 & 0 & 0 & 0 \\
\hline Überlebende & 34 & 9,65 & 6,00 & 13,80 & 2,00 \\
\hline Verstorbene & 1 & 7,00 & 7,00 & 7,00 & 0 \\
\hline
\end{tabular}

Der Hämatokritwert bei Aufnahme im Schockraum konnte bei 49 Patienten $(94,23 \%)$ der Gruppe 1 und bei 33 (84,62\%) der Gruppe 2 ausgewertet werden. Der Mittelwert in Gruppe 1 betrug 26,80\% \pm 8,38, in der Gruppe 2 betrug er 28,25\% $\pm 5,76$. Ein signifikanter Unterschied zwischen den zwei Gruppen fand sich bei der Auswertung des Hämatokritwertes nicht $(p=0,652)$.

Tabelle 11: Hämatokritwert bei Klinikaufnahme nach Schweregradgruppierung unterteilt für Gruppe 1.

\begin{tabular}{|l|l|l|l|l|l|}
\hline & $\mathrm{N}$ & Mittelwert (\%) & Minimum (\%) & Maximum (\%) & $\begin{array}{l}\text { Standard- } \\
\text { abweichung }\end{array}$ \\
\hline ISS 16-25 & 23 & 31,39 & 11,30 & 42,40 & 6,75 \\
\hline ISS 26-35 & 17 & 23,39 & 10,40 & 36,30 & 7,21 \\
\hline ISS 36-45 & 3 & 22,60 & 8,60 & 30,50 & 12,15 \\
\hline ISS 46-55 & 4 & 17,65 & 10,30 & 24,70 & 7,55 \\
\hline ISS $\geq 55$ & 2 & 27,50 & 26,60 & 28,40 & 1,28 \\
\hline Überlebende & 39 & 28,06 & 10,30 & 42,40 & 8,35 \\
\hline Verstorbene & 10 & 21,86 & 8,60 & 29,90 & 6,85 \\
\hline
\end{tabular}


Tabelle 12: Hämatokritwert bei Klinikaufnahme nach Schweregradgruppierung unterteilt für Gruppe 2.

\begin{tabular}{|l|l|l|l|l|l|}
\hline & $\mathrm{N}$ & Mittelwert (\%) & Minimum (\%) & Maximum (\%) & $\begin{array}{l}\text { Standard- } \\
\text { abweichung }\end{array}$ \\
\hline ISS 16-25 & 16 & 30,46 & 20,50 & 36,40 & 5,21 \\
\hline ISS 26-35 & 12 & 26,38 & 17,60 & 38,80 & 6,51 \\
\hline ISS 36-45 & 4 & 25,45 & 20,80 & 28,30 & 3,39 \\
\hline ISS 46-55 & 1 & 26,50 & 26,50 & 26,50 & 0 \\
\hline ISS $\geq 55$ & 0 & 0 & 0 & 0 & 0 \\
\hline Überlebende & 32 & 28,48 & 17,60 & 38,80 & 5,70 \\
\hline Verstorbene & 1 & 20,80 & 20,80 & 20,80 & 0 \\
\hline
\end{tabular}

\subsubsection{Stationäre Aufenthaltsdauer und Entlassung}

Bei 38 Patienten (73,08\%) der Gruppe 1 konnte in den Akten ein Aufnahme- und Entlassungsdatum entnommen werden, so dass ein gesamtstationäre Aufenthaltsdauer in der Universitätsmedizin Göttingen errechnet werden konnte. Der Mittelwert der Gruppe 1 betrug 48,71 Tage \pm 26,56. In der Gruppe 2 konnte bei 36 Patienten (92,31\%) die Aufenthaltsdauer errechnet werden, der Mittelwert betrug hier 47,39 Tage $\pm 38,63$. Ein signifikanter Unterschied zwischen den zwei Gruppen war nicht nachweisbar $(p=0,24)$.

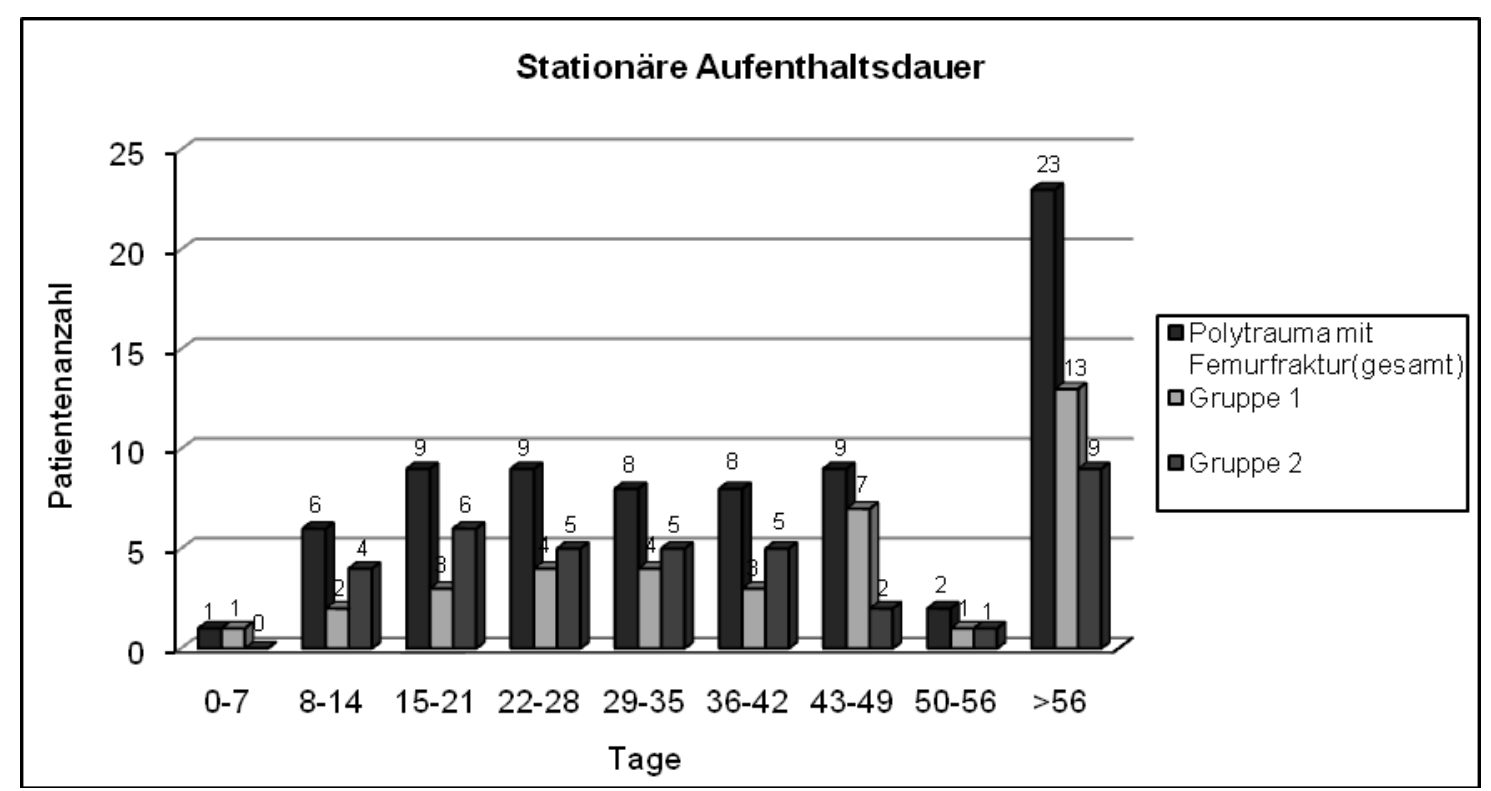

Abbildung 36: Stationäre Aufenthaltsdauer.

Bei 37 Patienten (71,15\%) der Gruppe 1 konnte in den vorliegenden Akten ein Entlassungsziel erfasst werden, dies erfolgte bei 35 Patienten $(89,74 \%)$ der Gruppe 2. In der nachfolgenden Abbildung sind die Entlassungsziele der zwei Gruppen aufgeführt, hierbei zeigte sich kein signifikanter Unterschied $(p=0,202)$. 


\section{Ergebnisse}

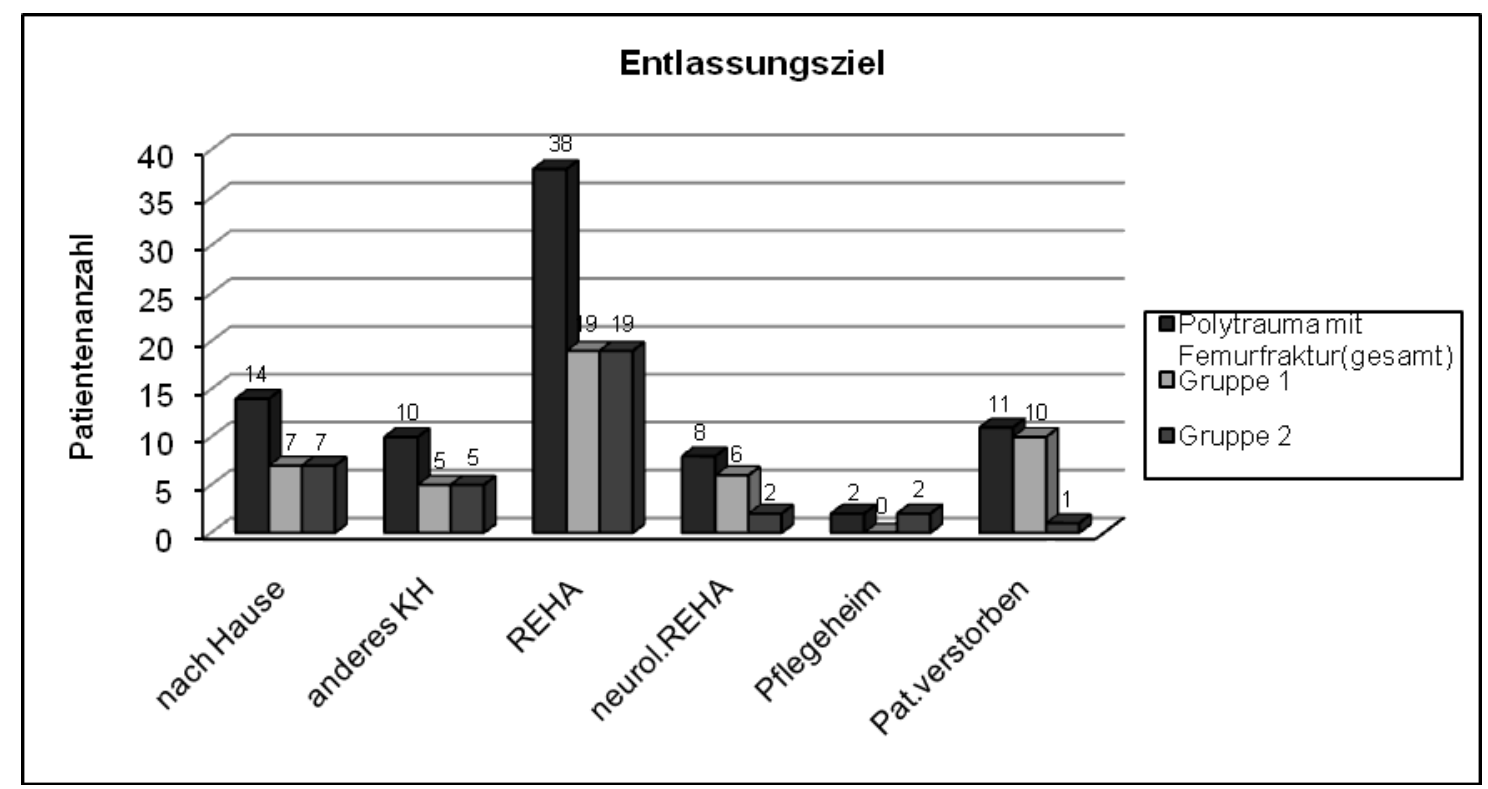

Abbildung 37: Entlassungsziel.

3.4.13. Komplikationen und Risikofaktoren

Im Hinblick auf die erfassten Komplikationen zeigte sich mit $p=0,026$ bei den allgemeinen Komplikationen ein signifikanter Unterschied zwischen den beiden Gruppen, der auch in der nachfolgenden Abbildung erkennbar ist. Bei 13 Patienten (25\%) der Gruppe 1 und bei 12 Patienten (30,77\%)der Gruppe 2 waren allgemeine Komplikationen aufgetreten.

Bei lokalen Komplikationen im Bereich des Femurs zeigte sich zwischen den beiden Gruppen kein signifikanter Unterschied ( $p=0,73$ ). Bei 11,54\% der Gruppe 1 und 17,95\% der Gruppe 2 kam es zu lokalen Komplikationen.

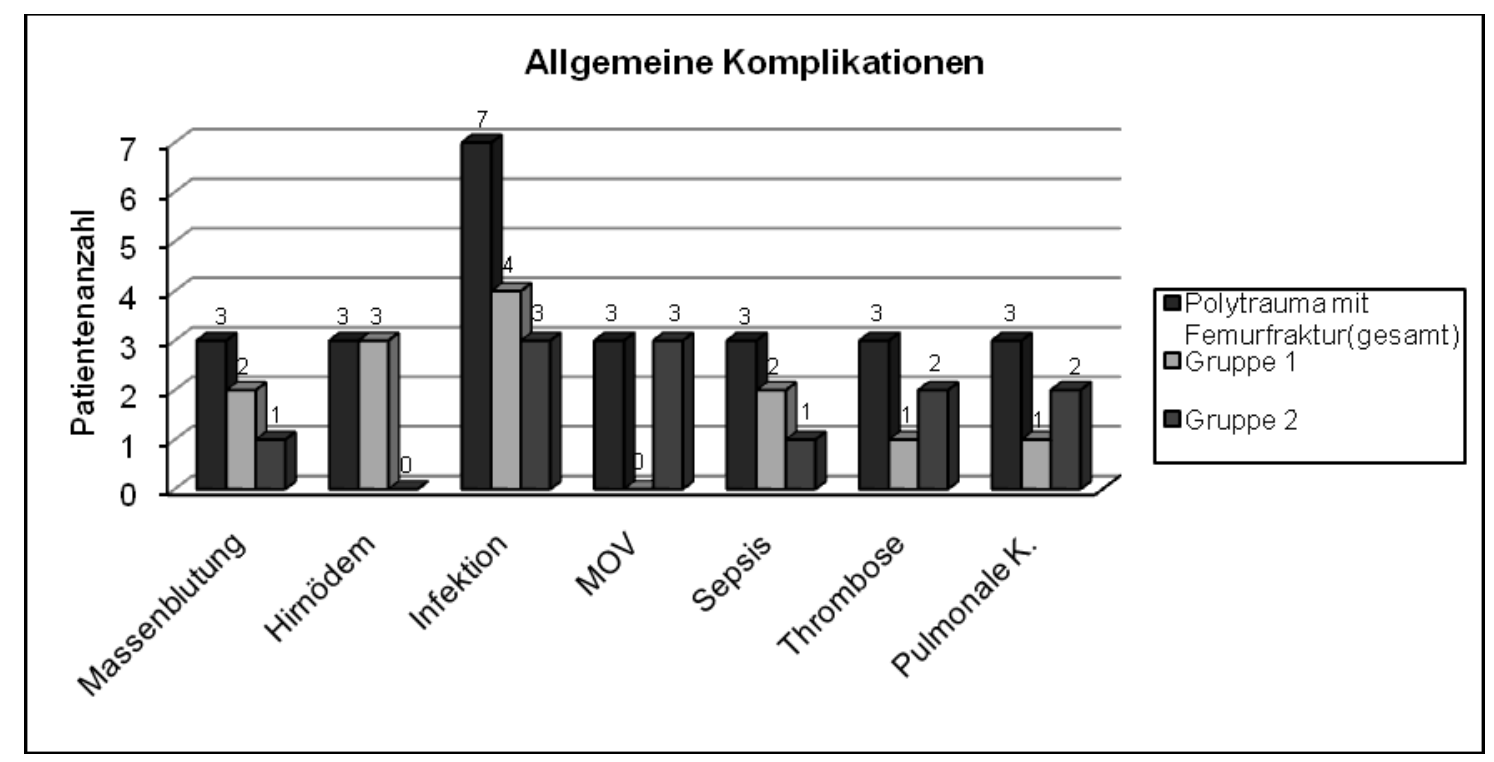

Abbildung 38: Allgemeine Komplikationen. 


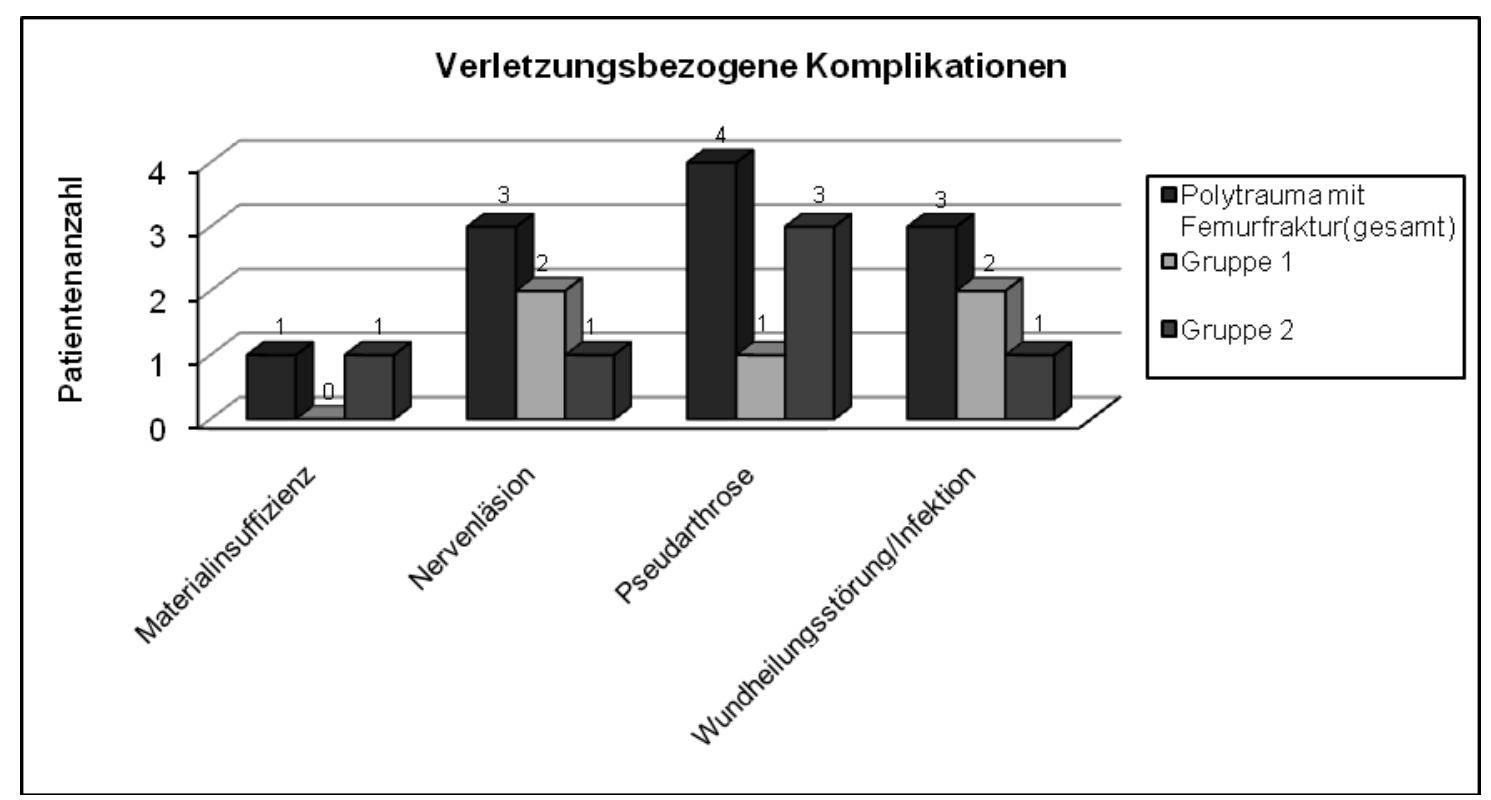

Abbildung 39: Lokale Komplikationen .

Bei je 18 Patienten (34,62\%) der Gruppe 1 und Gruppe 2 (46,15\%) konnten Risikofaktoren laut Akte erfasst. Im Vergleich zeigte sich kein signifikanter Unterschied mit $p=0,73$. In der nachfolgenden Abbildung sind die erfassten Risikofaktoren aufgeführt.

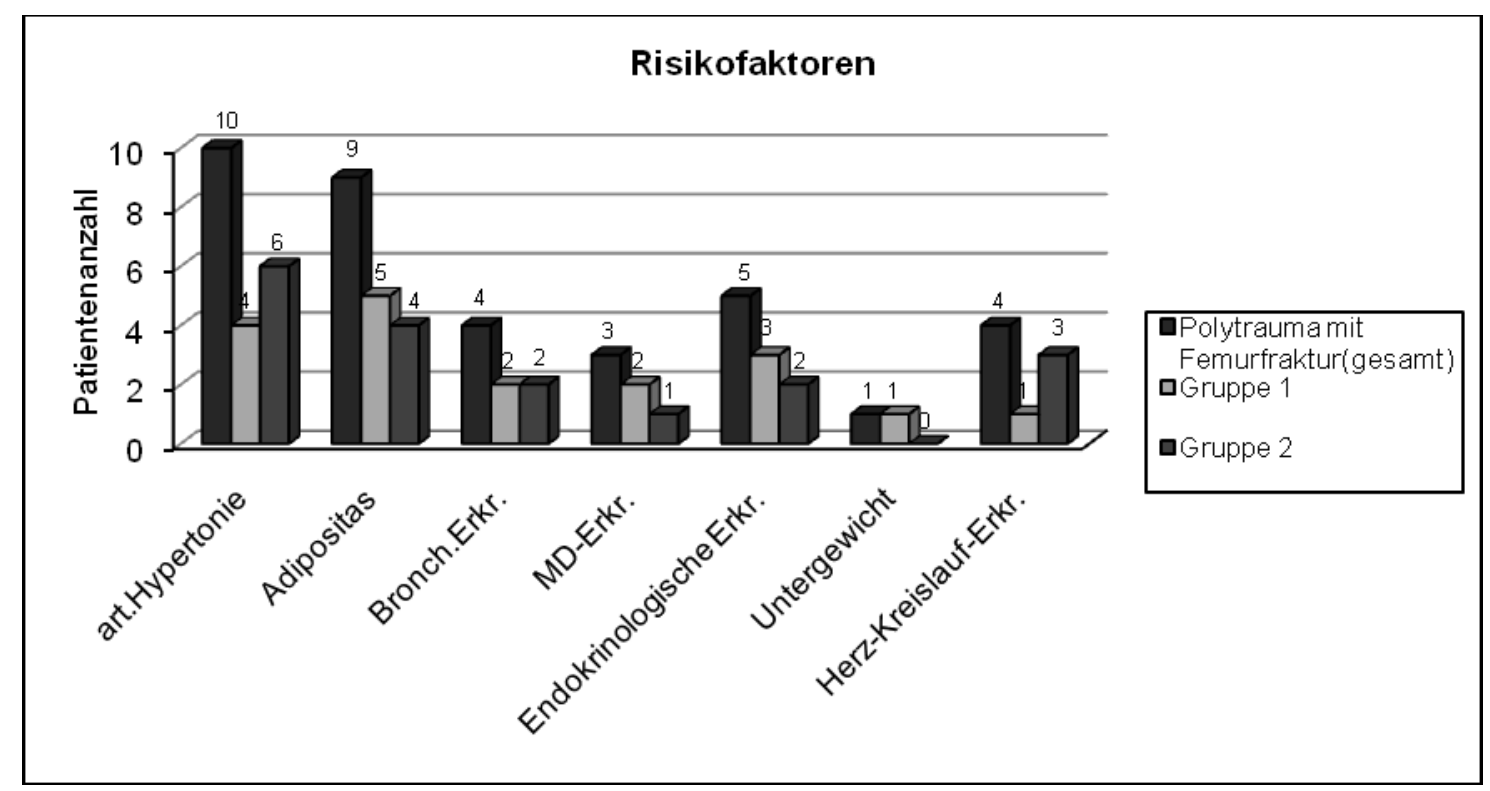

Abbildung 40: Risikofaktoren.

\subsection{Spätergebnisse}




\section{Ergebnisse}

An Hand der Fragebogenauswertung und der zusätzlichen klinischen Untersuchung wurde die hier vorliegende Studie über polytraumatisierte Patienten mit Femurfraktur vervollständigt. Von den insgesamt in die Studie einbezogenen 91 Polytraumapatienten mit Femurfraktur waren durch die Verletzungsschwere insgesamt 11 Patienten während des stationären Aufenthaltes verstorben. Deshalb konnten für die Nachuntersuchung bzw. Befragung nur 80 Patienten $(87,91 \%)$ angeschrieben werden. 7 Patienten (16,67\%) der Gruppe 1 und 6 Patienten (15,79\%) der Gruppe 2 waren leider unbekannt verzogen. Aus der Gruppe 2 waren im weiteren Verlauf nach der Entlassung zwei weitere Patienten (5,26\%) an unfallunabhängigen Ursachen verstorben.

\subsubsection{Auswertung des Fragebogens}

\subsubsection{Anzahl der Patienten und Nachuntersuchungszeitraum}

29 Patienten (69,05\%) der Gruppe 1, denen ein Fragebogen zugesandt worden war, beantworteten den zugeschickten Fragebogen, sowie 23 Patienten (63,89\%) der Gruppe 2, weitere 6 Patienten (14,29\%) der Gruppe 1 waren zur Beantwortung nicht bereit, ebenfalls 7 Patienten (19,44\%) der Gruppe 2.

Das Zeitintervall vom Unfallzeitpunkt bis zur Beantwortung des Fragebogens bzw. der klinischen Nachuntersuchung betrug zwischen einem und 8 Jahren. Der Mittelwert dieses Zeitintervalls in Gruppe 1 betrug 5,54 Jahre \pm 1,72, in Gruppe 2 betrug der Mittelwert 4,86 Jahre $\pm 1,76$.

\subsubsection{Soziodemographische Daten}

Die 2.Frage des erstellten Fragebogens beschäftigte sich mit dem Schulabschluss der Patienten. Zwischen den beiden Gruppen gab es keinen signifikanten Unterschied $(p=0,728)$. In der nachfolgenden Abbildung ist die Verteilung auf die einzelnen Schulabschlüsse aufgezeigt. 


\section{Ergebnisse}

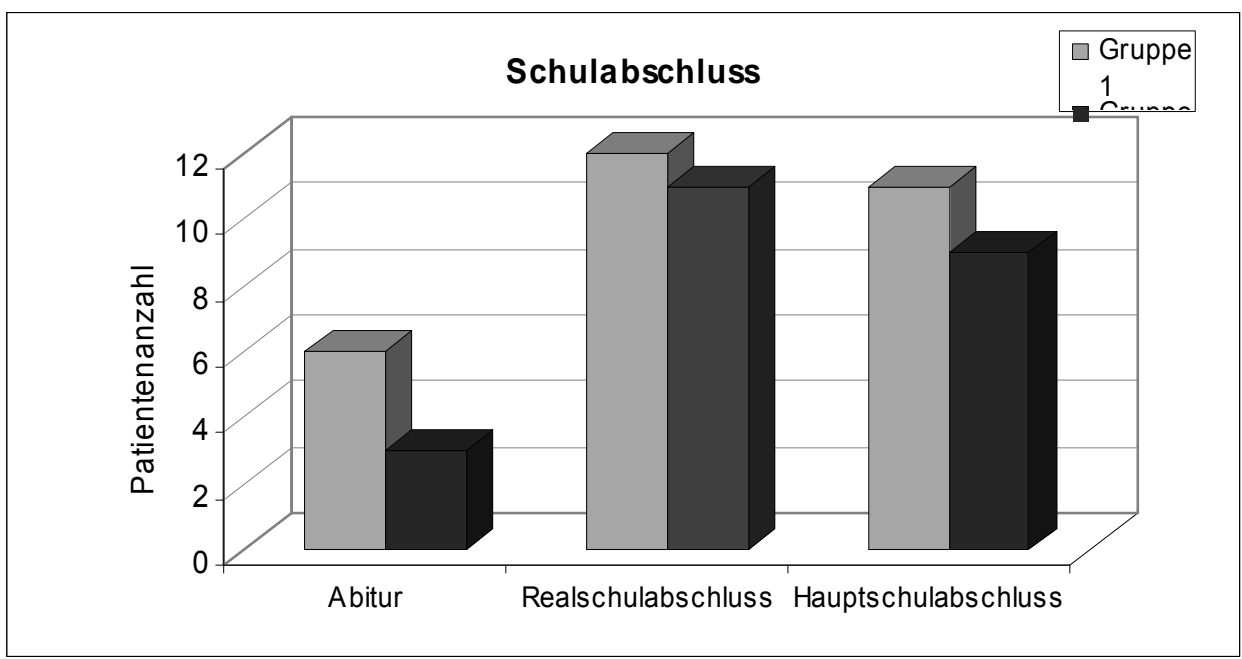

Abbildung 41: Abgeschlossener Schulabschluss der nachuntersuchten Patienten der Gruppe 1 und 2.

Die 3.Frage beschäftigte sich mit der absolvierten Berufsausbildung der Patienten, ein signifikanter Unterschied zwischen den Gruppen fand sich nicht $(p=0,534)$. In der nachfolgenden Abbildung ist die Berufsausbildung aufgeführt, es zeigt sich dass in beiden Gruppen eine große Anzahl Patienten eine Lehre abgeschlossen hatten.

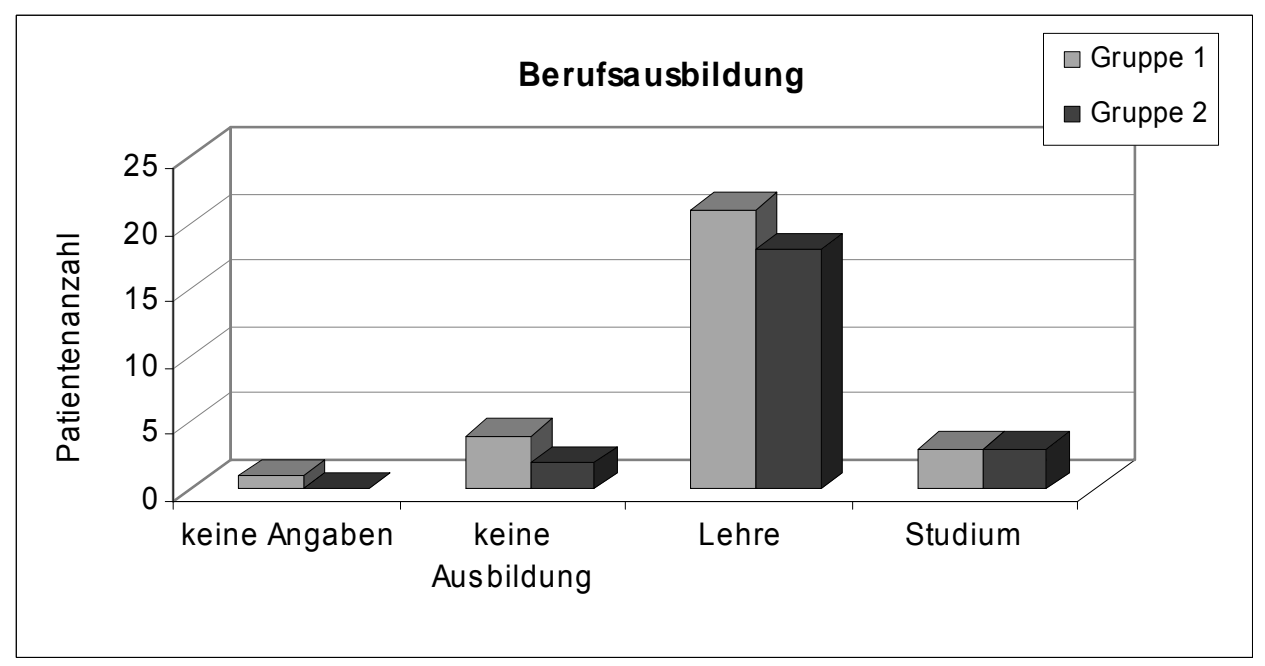

Abbildung 42: Abgeschlossene Berufsausbildung der befragten Patienten der Gruppe 1 und 2.

Auf die Frage nach der aktuellen Berufstätigkeit antworteten 18 Patienten der Gruppe 1 mit ja und 11 mit nein, aus der Gruppe 2 beantworteten 9 die Frage mit ja und 14 mit nein, ein signifikanter Unterschied zwischen den beiden Gruppen war jedoch nicht 
nachweisbar $(p=0,10)$. In den nachfolgenden zwei Abbildungen ist dies graphisch dargestellt.
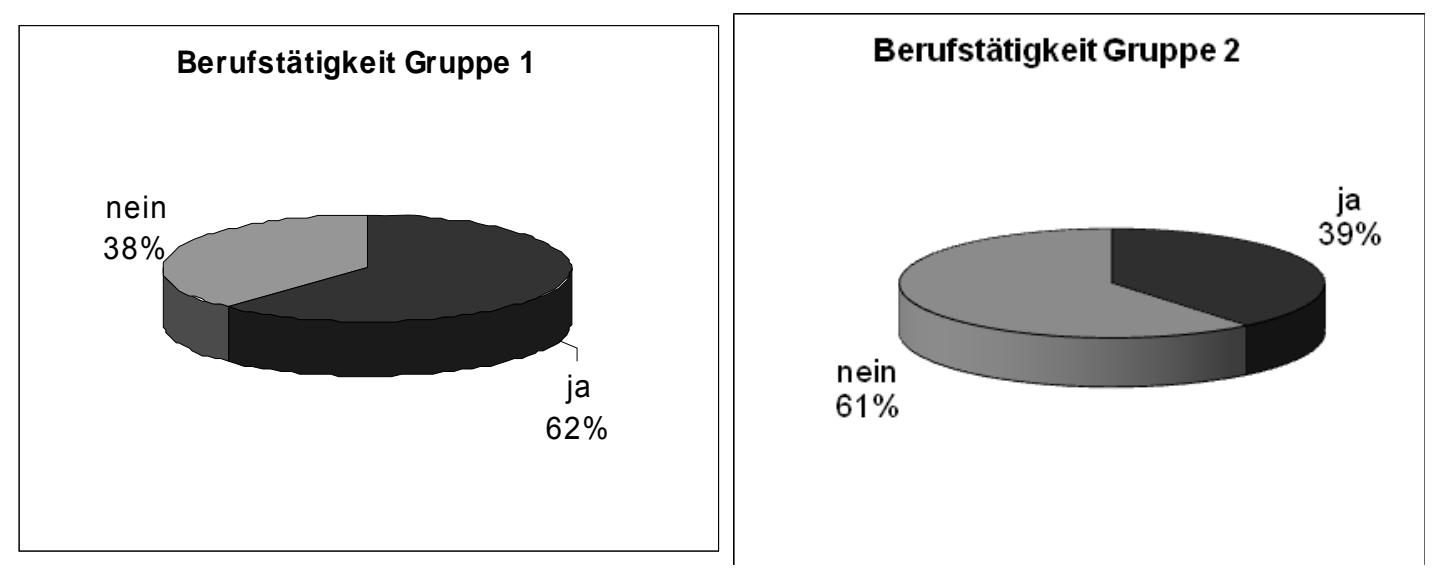

Abbildungen 43/44: Berufstätigkeit der befragten Patienten in Gruppe 1 und 2.

Patienten, die die Frage nach der Berufstätigkeit mit nein beantwortet hatten, hatten in der nachfolgenden Frage die Möglichkeit der Angabe, was sie stattdessen erhalten bzw. ob sie z.B. eine Umschulungsmaßnahme durchführen. Bei denjenigen, die mit ja geantwortet hatten, erfolgte eine weitere Frage nach dem Beruf, d.h. ob der gleiche wie vor dem Unfall möglich ist, oder ob sie einer anderen Tätigkeit nachgehen. Bei beiden Unterfragen gab es hinsichtlich der beiden Gruppen keinen signifikanten Unterschied $(p=0,267)$. In der nachfolgenden Abbildung ist die Verteilung zusammengefasst aufgezeigt.

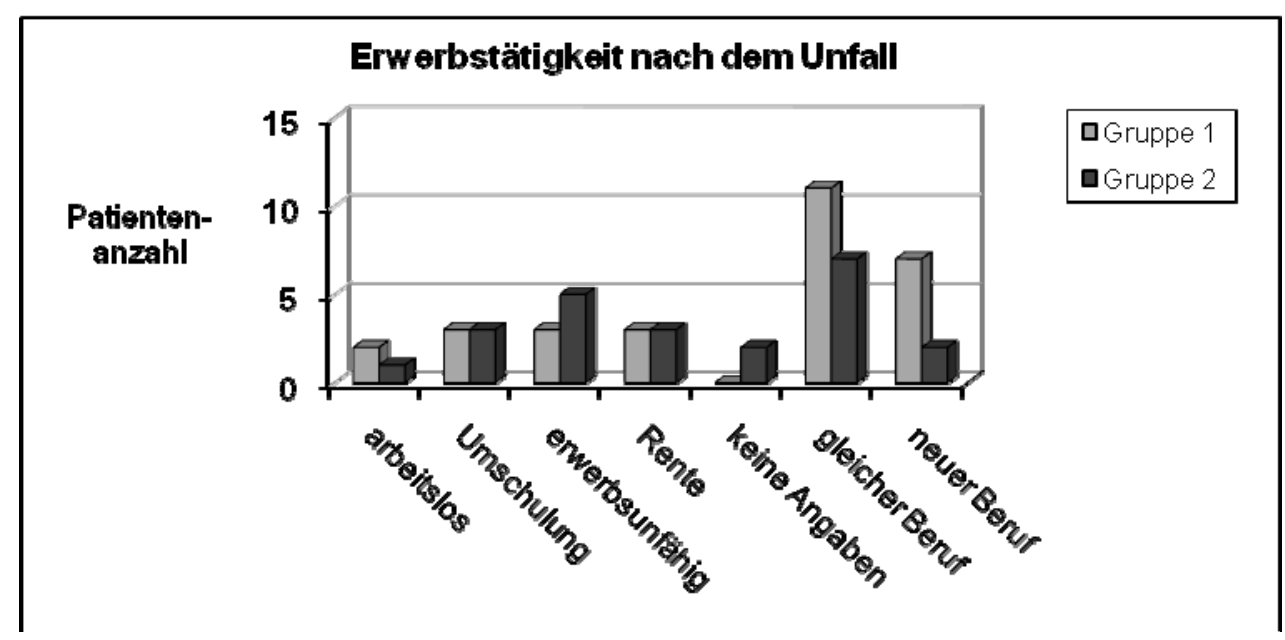

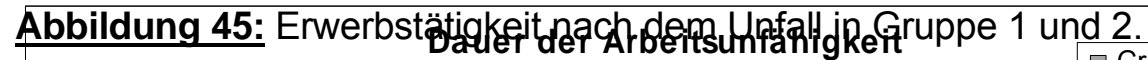

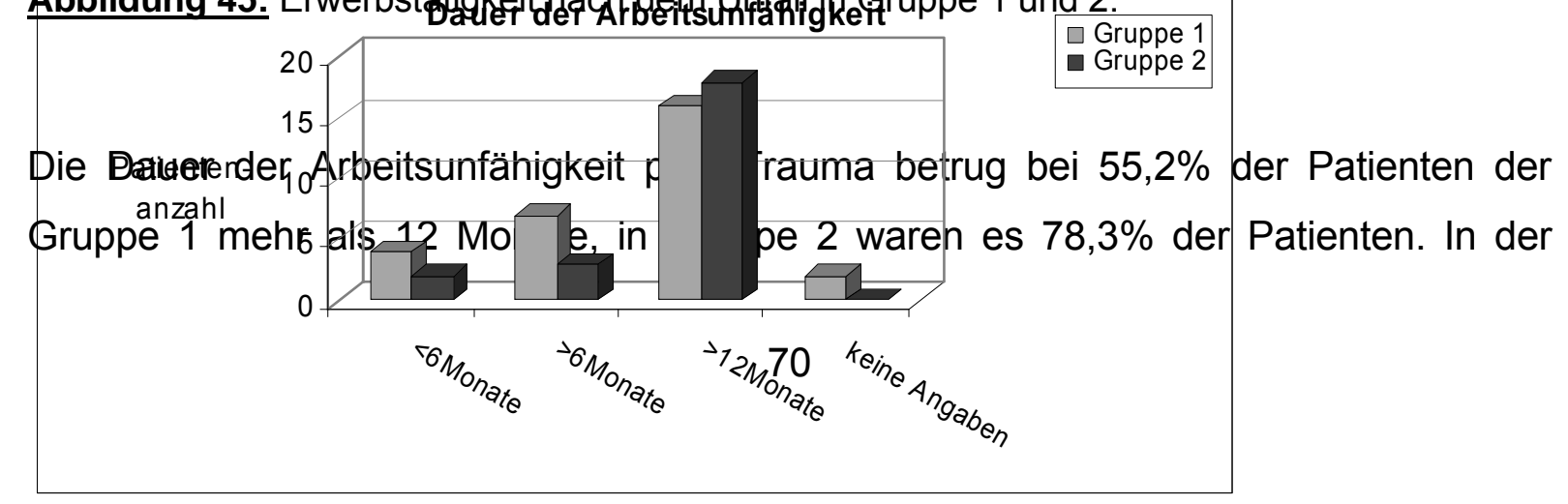


nachfolgenden Abbildung findet sich die Verteilung der Arbeitsunfähigkeitszeiten. Ein signifikanter Unterschied zwischen den Gruppen fand sich nicht $(p=0,522)$.

Abbildung 46: Arbeitsunfähigkeitszeiten in Gruppe 1 und 2 nach dem Trauma.

Bei 24 Patienten $(82,76 \%)$ der Gruppe 1 wurde im Anschluss an den stationären Aufenthalt eine AHB -Maßnahme durchgeführt, in Gruppe 2 ist dies bei 17 Patienten $(73,91 \%)$ erfolgt. Der größte Anteil der Patienten der Gruppe $1(37,5 \%)$, welche eine AHB durchführten, hatten eine Aufenthaltsdauer in der AHB von 4 Wochen, mit 47,1\%

der Patienten der Gruppe 2 war der größte Anteil mit einer Aufenthaltsdauer von 6 Wochen in der AHB, ein signifikanter Unterschied zwischen den zwei Gruppen zeigte sich jedoch nicht $(p=0,211)$.

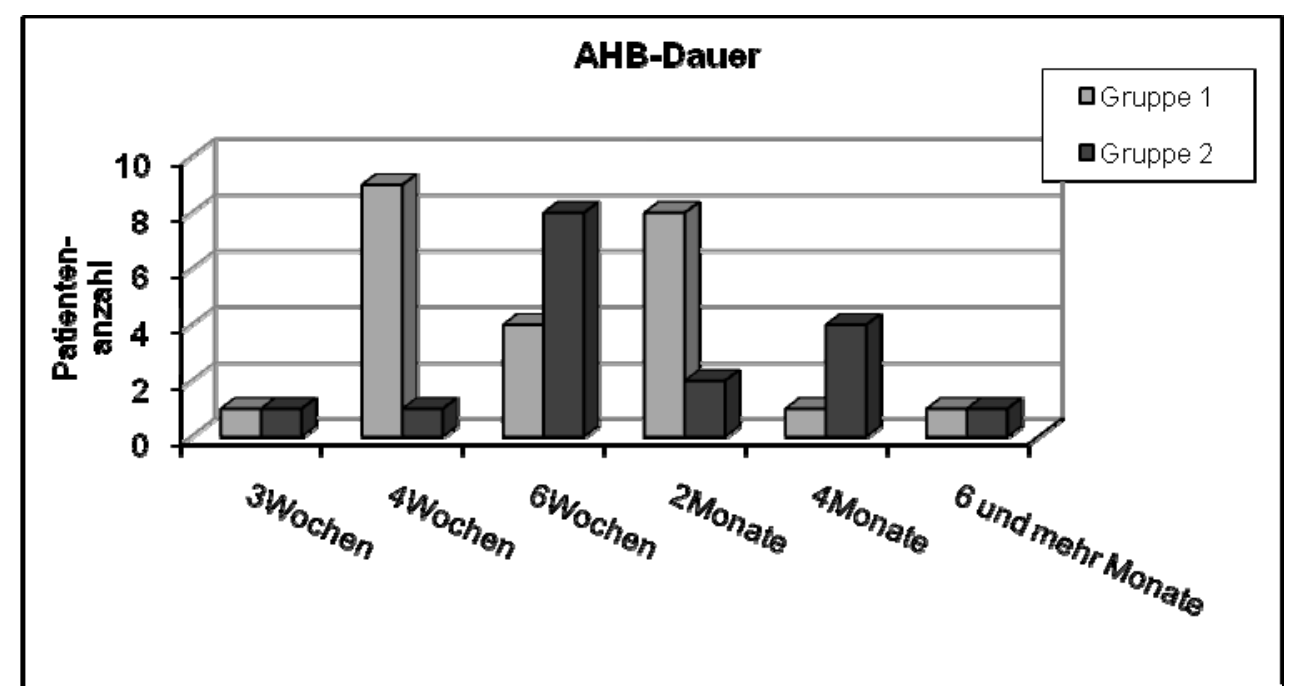

Abbildung 47: AHB-Aufenthaltsdauer in Gruppe 1 und Gruppe 2.

17,24\% der befragten Patienten der Gruppe 1 erhalten eine private Unfallrente, $31,03 \%$ eine berufsgenossenschaftliche Rente. Im Vergleich hierzu erhalten $21,74 \%$ 
der befragten Patienten der Gruppe 2 eine private Unfallrente und 34,78\% eine berufsgenossenschaftliche Rente. Einen signifikanten Unterschied mit $p=0,66$ gab es nicht.

\subsubsection{Veränderungen im privaten Umfeld sowie der Lebensumstände}

Bei $72,41 \%$ der Patienten der Gruppe 1 war keine Veränderung der häuslichen Umgebung notwendig, im Vergleich hierzu war nur bei $47,83 \%$ der Patienten der Gruppe 2 keine Veränderung erforderlich. Ein signifikanter Unterschied zeigte sich jedoch beim Vergleich der beiden Gruppen nicht $(p=0,14)$.

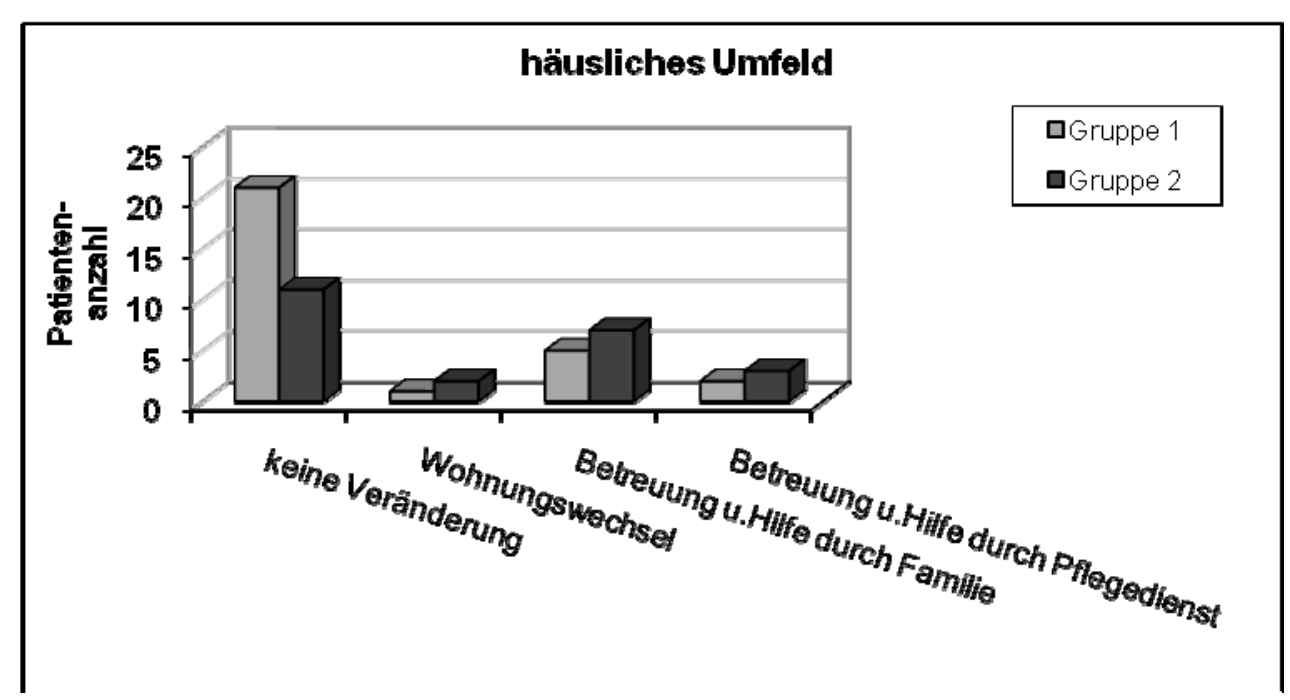

Abbildung 48: Veränderungen im häuslichen Umfeld nach dem Trauma in Gruppe 1 und 2.

Nach dem Trauma ist bei 51,72\% der Patienten der Gruppe 1 das Hobby gleich geblieben. Im Vergleich hierzu ist 52,17\% der Patienten der Gruppe 2 ihr zuvor ausgeübtes Hobby nicht mehr möglich. In der nachfolgenden Abbildung ist dies nochmals dargestellt, ein signifikanter Unterschied fand sich nicht $(p=0,42)$. 


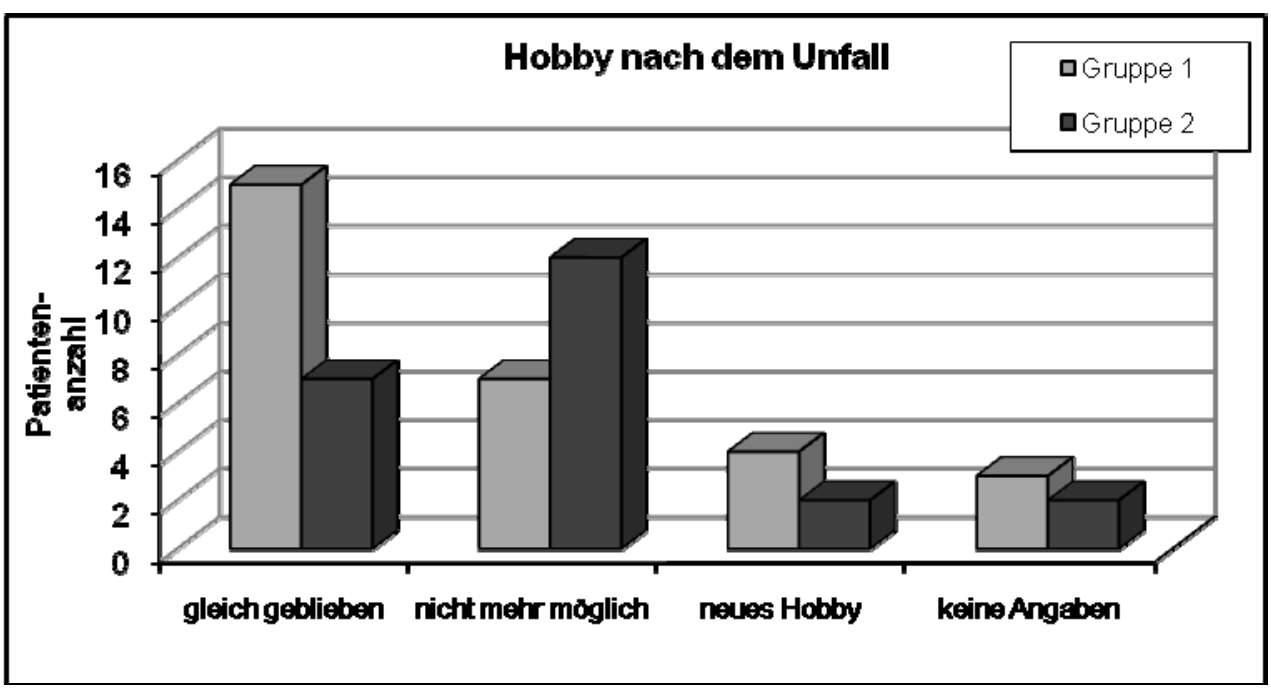

Abbildung 49: Veränderungen des Hobbys nach dem Unfall in Gruppe 1 und 2.

Auf die Frage nach der sportlichen Aktivität antworteten 41,38\% der Patienten der Gruppe 1, dass ihnen keine sportliche Aktivität nach dem Unfall mehr möglich ist. Hierzu antworteten 47,83 \% der Gruppe 2, dass eine sportliche Aktivität nicht mehr möglich ist. Beim Vergleich der beiden Gruppen zeigte sich mit $p=0,041$ ein signifikanter Unterschied vor allem im Hinblick darauf, dass in Gruppe 1 deutlich mehr Patienten ihrer sportlichen Aktivität wieder nachgehen.

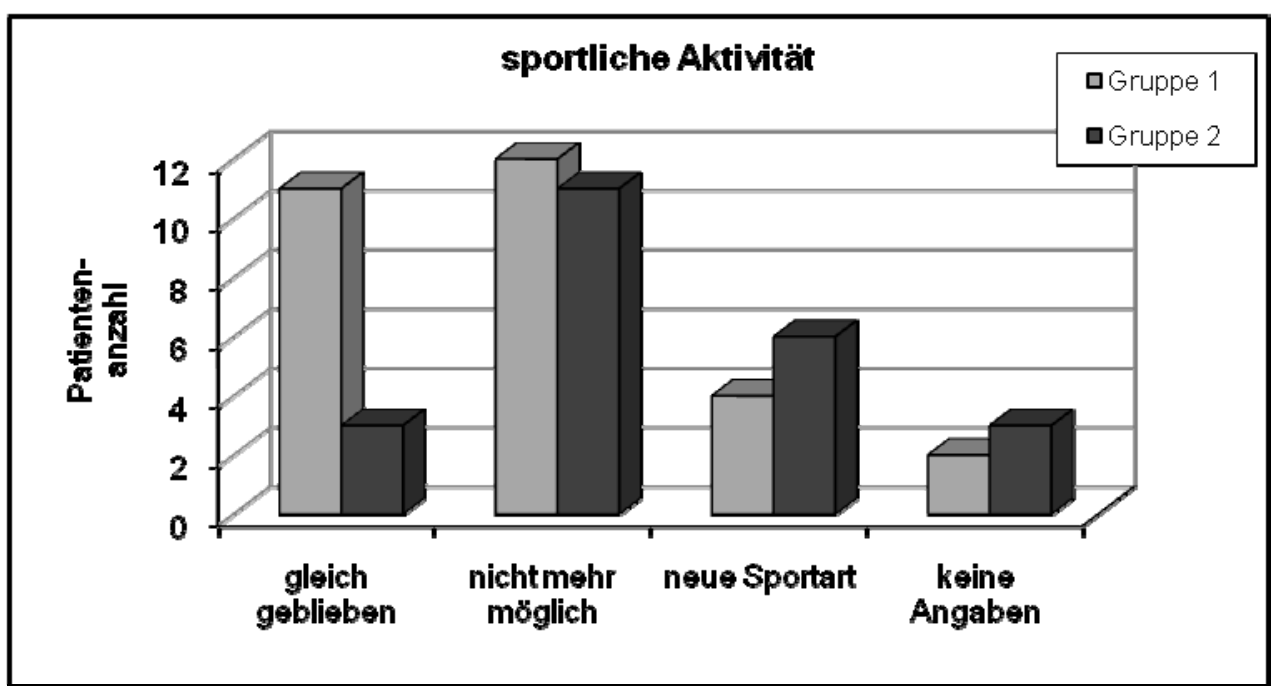

Abbildung 50: Veränderungen der sportlichen Aktivität nach dem Unfall in Gruppe 1 und 2.

$51,72 \%$ der befragten Patienten der Gruppe 1 sind in ihrer Urlaubsplanung und -ausübung eingeschränkt, dies trifft auch auf 47,83 \% der Patienten der Gruppe 2 zu. 
Ein signifikanter Unterschied zwischen den Gruppen konnte nicht festgestellt werden $(p=0,956)$.

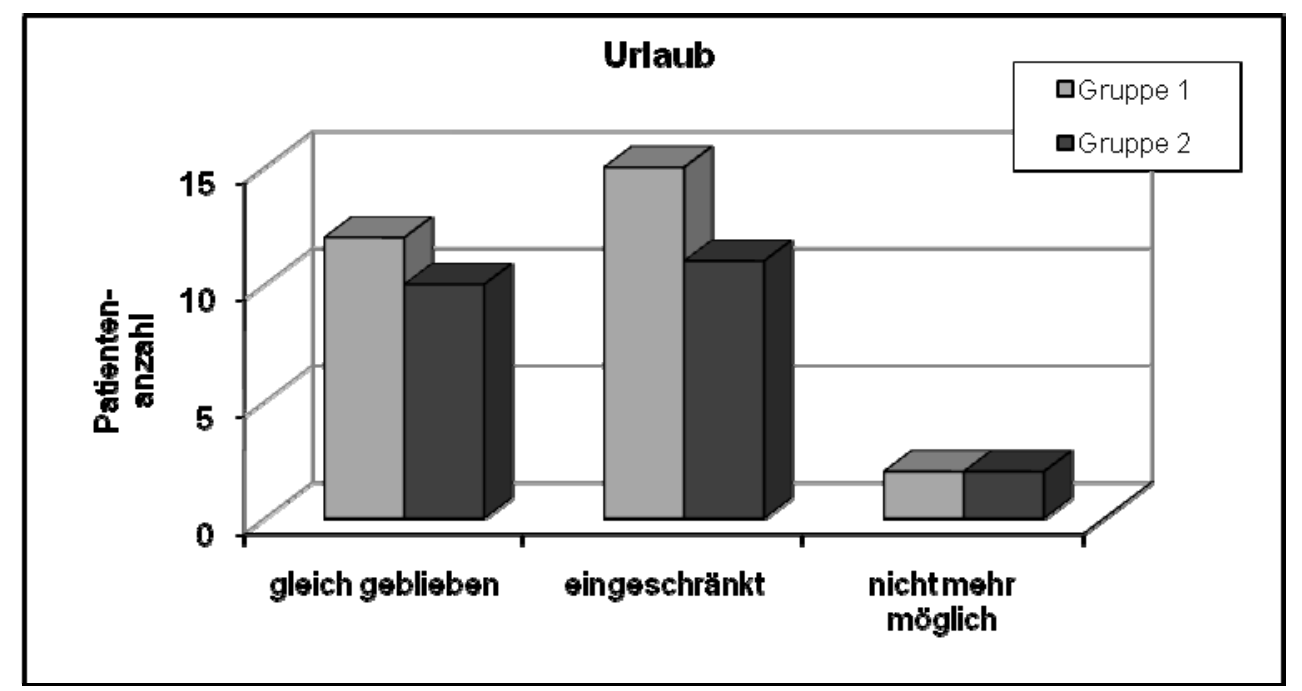

Abbildung 51: Veränderungen im Urlaubsverhalten in Gruppe 1 und 2.

\subsubsection{Aussagen zu Schmerzen nach dem Trauma}

72,41\% (21 Patienten) der befragten Patienten der Gruppe 1 gaben an, dass sie nach dem Unfall zum Befragungszeitpunkt noch Schmerzen haben. Im Vergleich hierzu waren es in Gruppe 2 78,26\% (18 Patienten). Ein signifikanter Unterschied zwischen den Gruppen war hierbei nicht nachweisbar $(p=0,63)$.

In der nachfolgenden Abbildung sind die verschiedenen geäußerten Schmerzstärken zum Vergleich aufgeführt. Ein signifikanter Unterschied mit $p=0,210$ zeigte sich nicht.

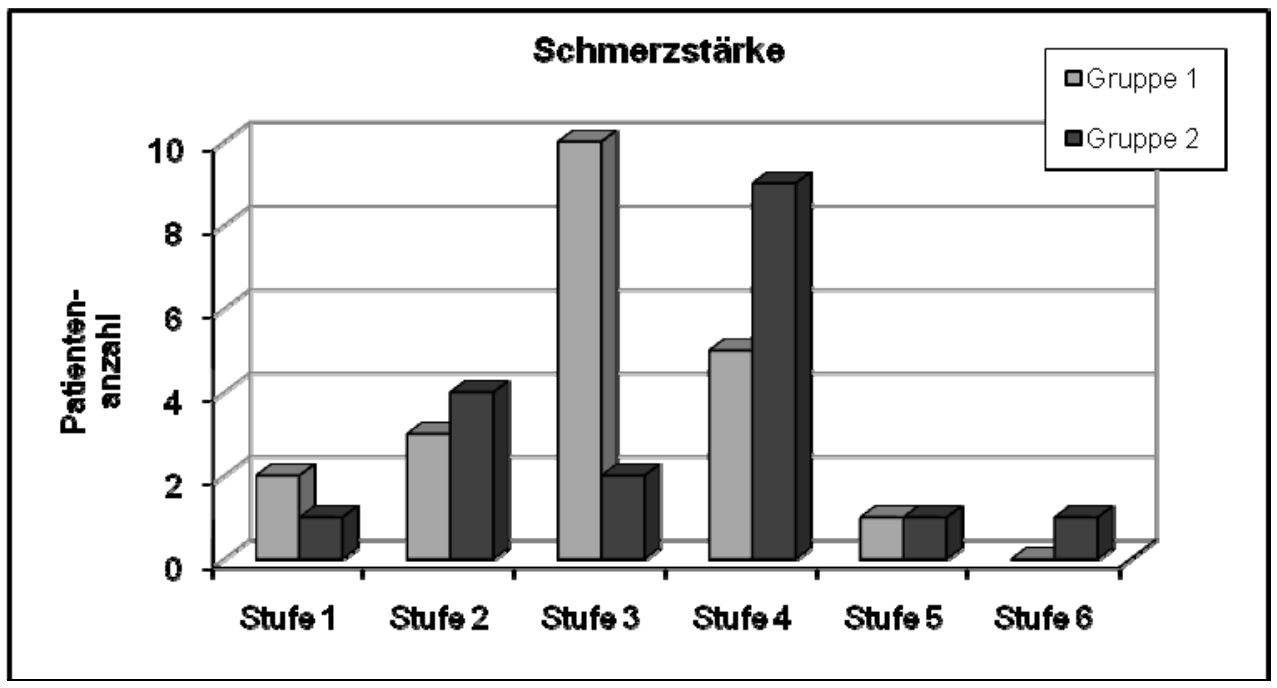


Abbildung 52: Schmerzintensität nach dem Trauma in Gruppe 1 und 2.

In der folgenden Abbildung ist die Häufigkeit des Schmerzauftretens der beiden zu untersuchenden Gruppen aufgeführt, auch hierbei zeigte sich mit $p=0,812$ kein signifikanter Unterschied zwischen den beiden Gruppen.

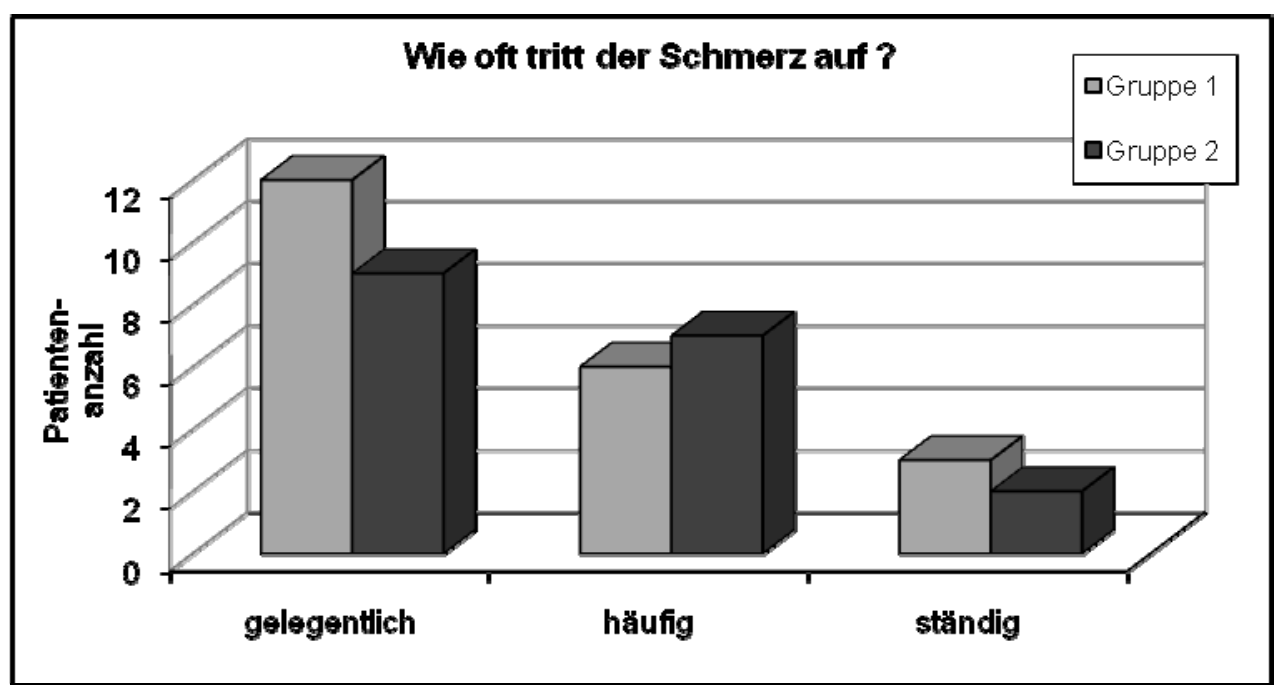

Abbildung 53: Schmerzhäufigkeit in Gruppe 1 und 2.

An Hand der nachfolgenden Abbildung ist der Schmerzverlauf des letzten halben Jahres vor der Untersuchung festgehalten, es zeigte sich dabei kein signifikanter Unterschied zwischen den Gruppen ( $p=0,834)$.

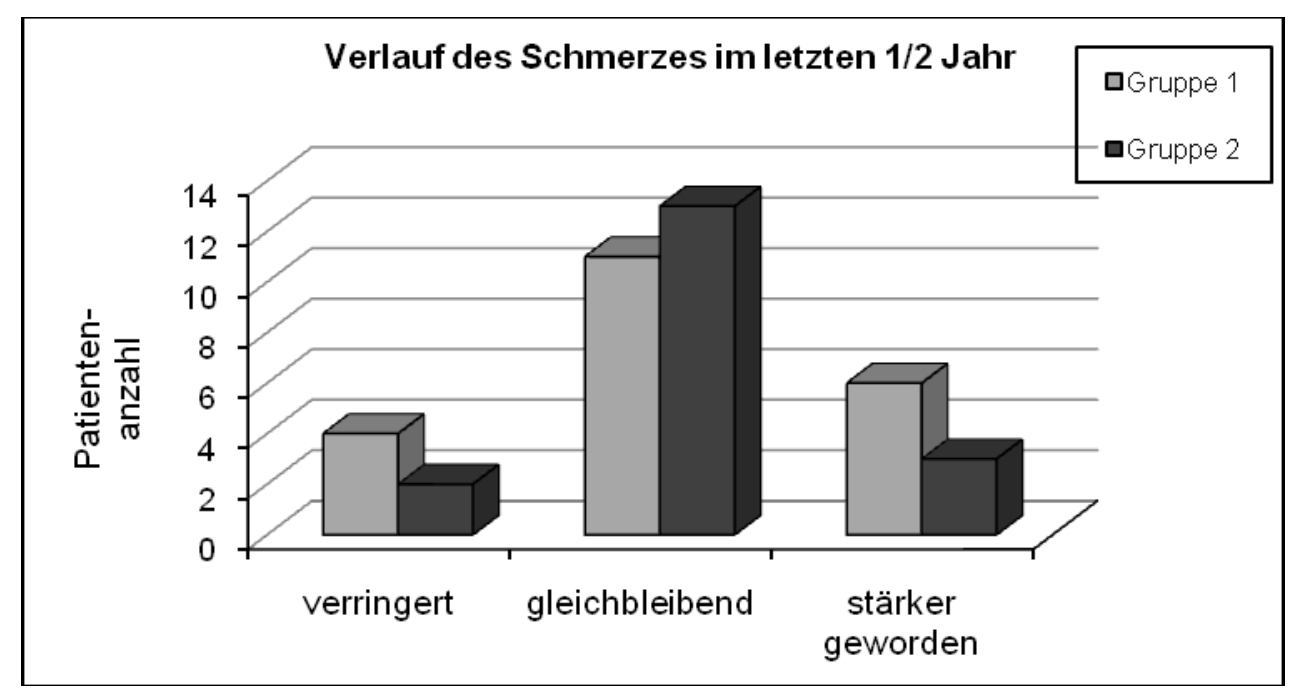

Abbildung 54: Schmerzverlauf im letzten halben Jahr vor der Befragung in Gruppe 1 und 2. 
43\% der Patienten der Gruppe 1 äußerten, dass sie keinen Ruhe- bzw. Nachtschmerz verspüren, im Vergleich hierzu haben $28 \%$ der Gruppe 2 keinen Ruhe- bzw. Nachtschmerz. In den nachfolgenden zwei Abbildungen ist die Ausprägung des Ruhebzw. Nachtschmerzes aufgezeigt. Mit $p=0,791$ zeigte sich kein signifikanter Unterschied zwischen den beiden Gruppen.

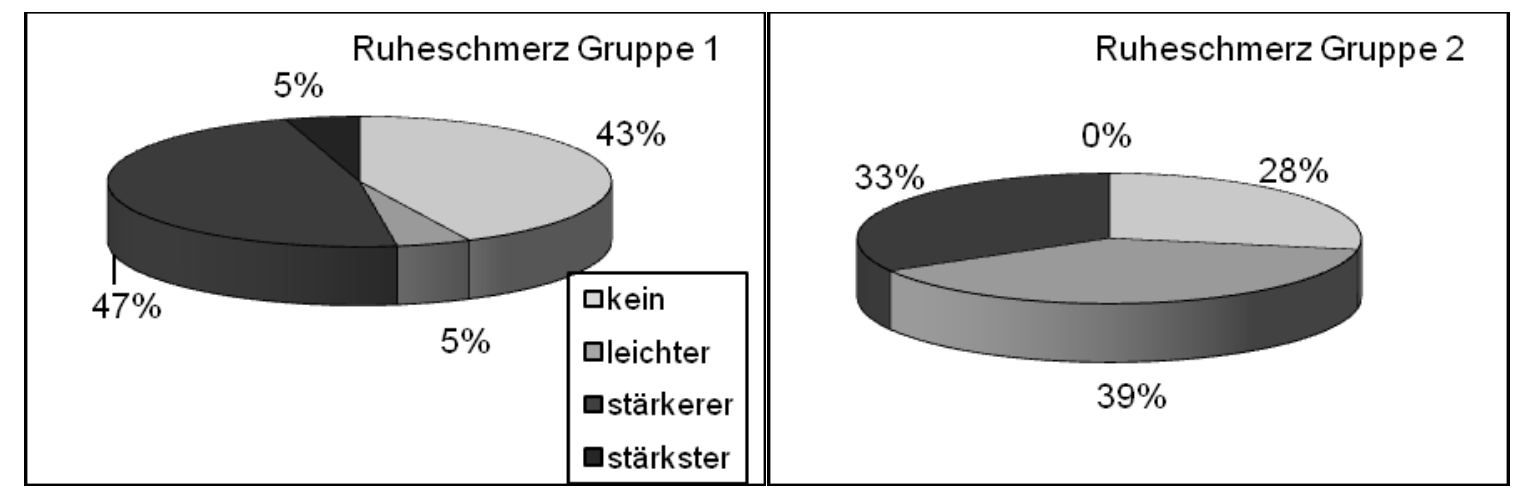

Abbildungen 55/56: Auftreten von Ruheschmerzen bzw. Nachtschmerzen in Gruppe 1 und 2.

76\% der Patienten mit Schmerzen der Gruppe 1 haben einen belastungs- bzw. bewegungsabhängigen Schmerz, im Vergleich hierzu haben $83 \%$ der Schmerzpatienten der Gruppe 2 einen Belastungs- bzw. Bewegungsschmerz. Ein signifikanter Unterschied in der Schmerzstärke zeigt sich nicht $(p=0,459)$.

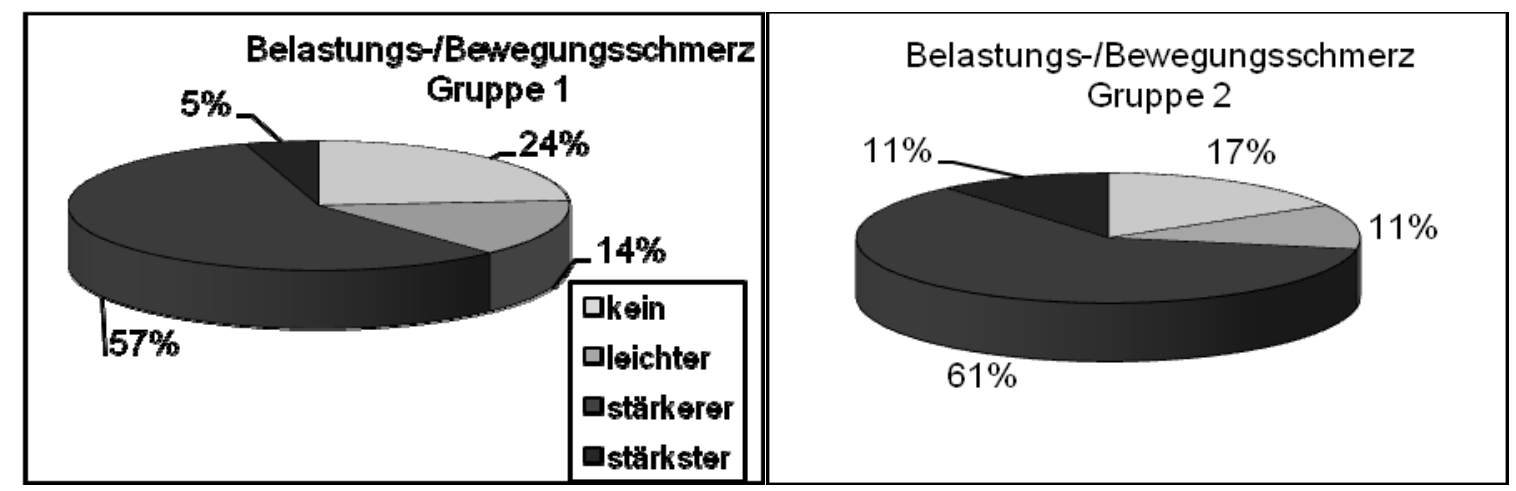

Abbildungen 57/58: Schmerzstärke des Belastungs- bzw. Bewegungsschmerzes in Gruppe 1 und 2. 
58\% der Patienten der Gruppe 1, die Schmerzen äußerten, gaben als Schmerzlokalisation unter anderem die betroffene untere Extremität an, ähnlich verhielt es sich auch in der Gruppe 2, wo 59\% Schmerzen im Bereich der betroffenen unteren Extremität angaben. In den nachfolgenden Abbildungen ist die Schmerzlokalisation in beiden Gruppen zum Vergleich aufgeführt, auch hier konnte kein signifikanter Unterschied $(p=0,807)$ gefunden werden.
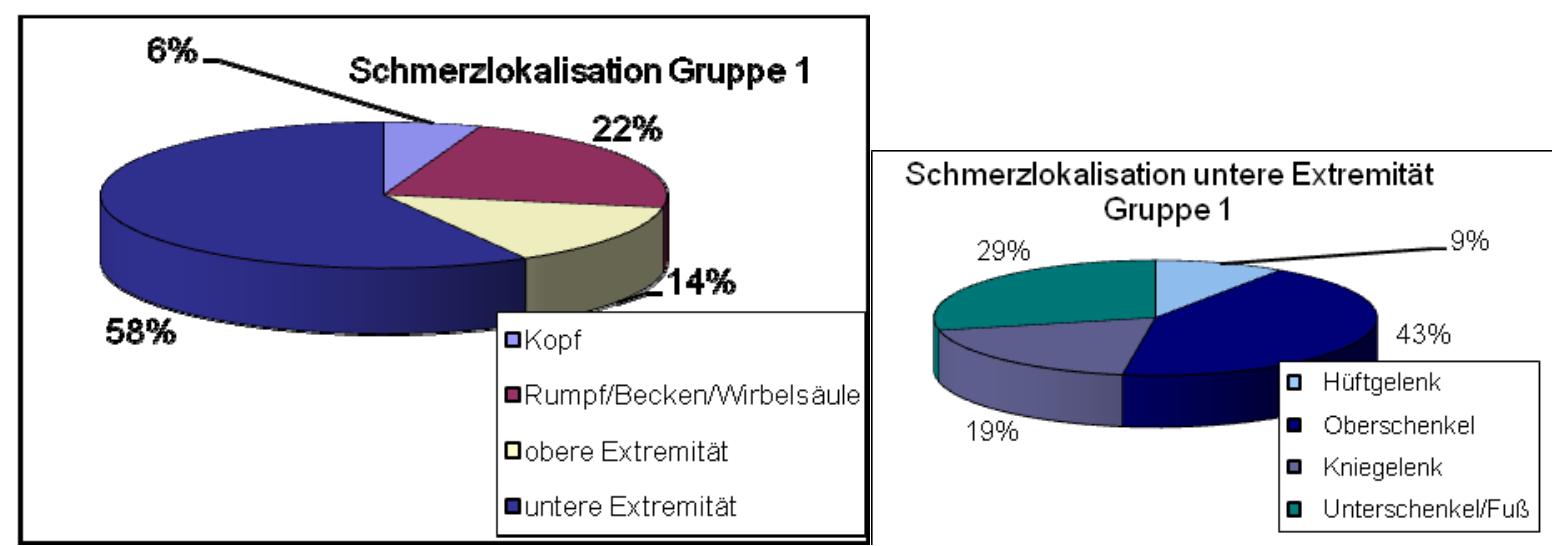

Abbildung 59/60: Schmerzlokalisation in Gruppe 1.

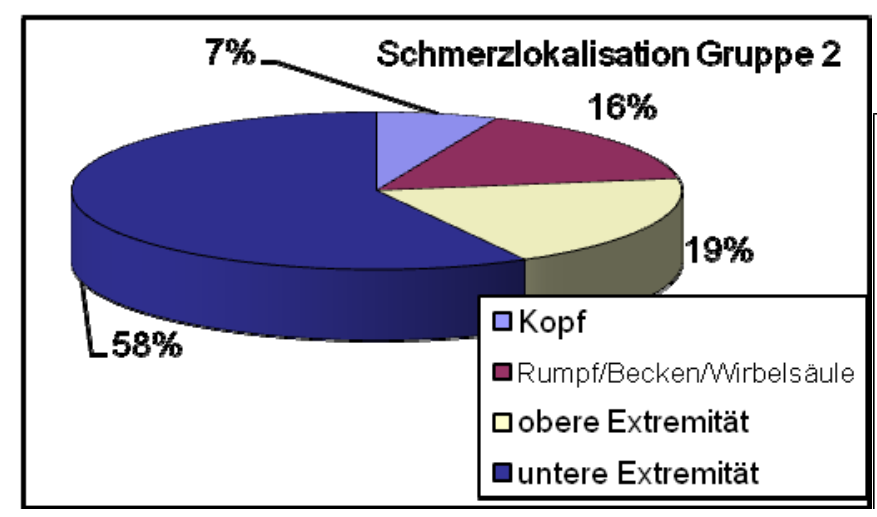

Schmerzlokalisation untere Extremität Gruppe 2

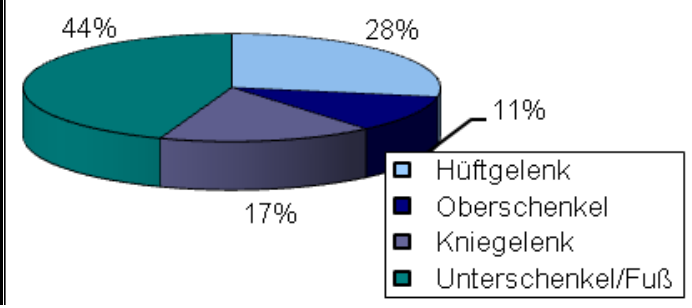

Abbildung 61/62: Schmerzlokalisation in Gruppe 2.

47,62\% der Patienten der Gruppe 1, die Schmerzen angaben, beschrieben den vorhandenen Schmerz als dumpf und drückend, im Vergleich hierzu beschrieben dies auch 44,44\% der Patienten der Gruppe 2. Kein Patient der Gruppe 1 hatte einen unerträglichen Schmerz, im Vergleich hierzu gab ein Patient der Gruppe 2 einen unerträglichen Schmerz an. Beim Vergleich der beiden Gruppen zeigte sich mit $p=0,586$ kein signifikanter Unterschied. In der nachfolgenden Abbildung sind die unterschiedlichen Schmerzqualitäten aufgeführt. 


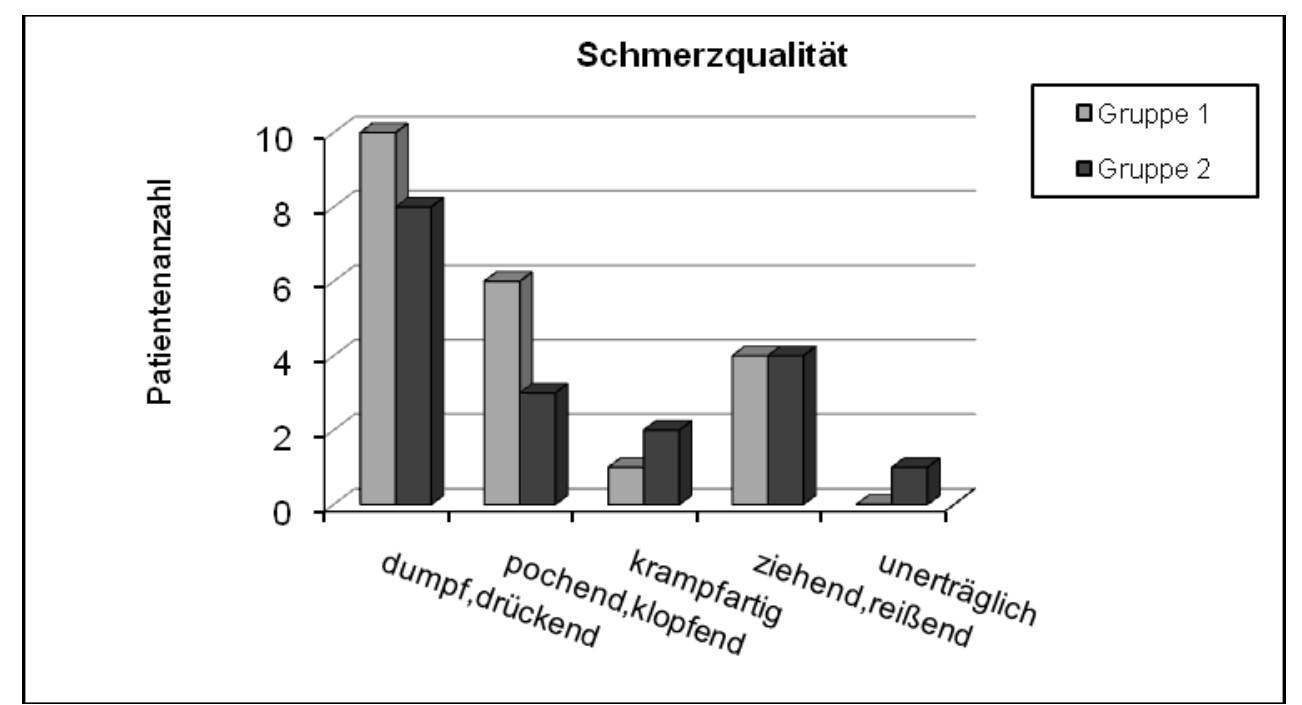

Abbildung 63: Schmerzqualität in Gruppe 1 und 2 .

Eine medikamentöse Schmerztherapie erhielten laut eigenen Angaben zum Nachuntersuchungszeitraum 38,10 \% der Patienten ( $N=8)$ mit Schmerz der Gruppe 1, bei Gruppe 2 waren es $50 \%$ der Patienten mit Schmerz $(\mathrm{N}=9)$. In der nachfolgenden Tabelle ist aufgeführt welche Medikation nach WHO gegen die Schmerzen eingenommen wurde.

Tabelle 12: Schmerzmedikation nach WHO-Schema in Gruppe 1 und 2.

\begin{tabular}{|c|c|c|}
$\begin{array}{c}\text { Schmerzmedikation nach } \\
\text { WHO }\end{array}$ & $\begin{array}{c}\text { Patientenanzahl der } \\
\text { Gruppe 1 } \\
\text { (prozentualer Anteil) }\end{array}$ & $\begin{array}{c}\text { Patientenanzahl der } \\
\text { Gruppe 2 } \\
\text { (prozentualer Anteil) }\end{array}$ \\
\hline WHO I & $5(62,5 \%)$ & $6(66,67 \%)$ \\
\hline WHO II & $2(25,0 \%)$ & $2(22,22 \%)$ \\
\hline WHO III & $1(12,5 \%)$ & $1(11,11 \%)$ \\
\hline
\end{tabular}

Eine Bedarfsmedikation ca. 1x monatlich gaben 37,5\% der Patienten mit Schmerzen der Gruppe 1 an, dagegen gaben 44,44\% der Patienten mit Schmerzen der Gruppe 2 ihre Schmerzmitteleinnahme mit 2-3 x wöchentlich an. Dementsprechend nahmen die Patienten der Gruppe 2 häufiger die Schmerzmedikation ein als die Patienten der Gruppe 1, ein signifikanter Unterschied zeigte sich jedoch nicht $(p=0,276)$.

37,5\% der Patienten der Gruppe 1, die Schmerzmittel bei Bedarf einnahmen, gaben jedoch nur eine kurzzeitige Linderung von ca. 1 Stunde an. In der Gruppe 2 gaben 
33,33\% der Patienten nur eine kurzzeitige Besserung an. Eine längere Besserung der Schmerzen von 5-12 Stunden durch die Schmerzmedikation gaben $25 \%$ der Patienten der Gruppe 1 an und 33,3\% der Gruppe 2.

\subsubsection{Belastbarkeit und Gehstrecke nach dem Trauma}

Die 10. Frage des Patientenfragebogens beschäftigte sich mit der Gehstrecke nach dem Unfall zum Befragungszeitpunkt. Eine deutliche Einschränkung der Gehstrecke gaben insgesamt mehr Patienten der Gruppe 2 im Vergleich zur Gruppe 1 an, ein signifikanter Unterschied konnte jedoch mit $p=0,11$ nicht gezeigte werden. In der nachfolgenden Abbildung sind die Angaben der befragten Patienten beider Gruppen gegenüber gestellt.

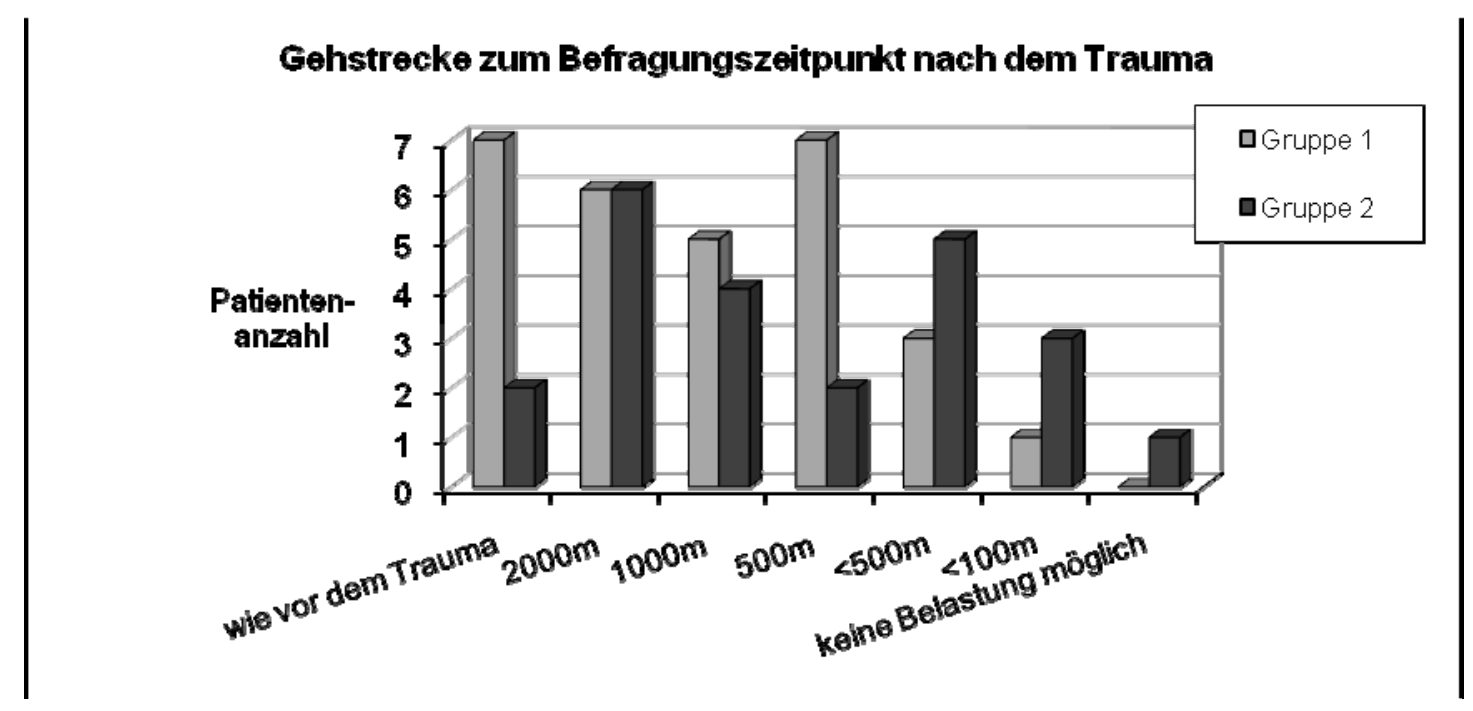

Abbildung 64: Gehstreckeneinschränkungen nach dem Trauma in Gruppe 1 und 2.

Hinsichtlich der körperlichen Belastbarkeit gaben 51,72\% ( $N=15)$ der Patienten der Gruppe 1 eine leichte Einschränkung an. In Gruppe 2 gaben 39,13\% (N=9) eine leichte Einschränkung der Belastbarkeit an. Ein signifikanter Unterschied zwischen den beiden Gruppen hinsichtlich der körperlichen Belastbarkeit nach dem Trauma konnte nicht gezeigt werden $(p=0,17)$. In der nachfolgenden Abbildung sind die die 
Einschränkungen in der Belastbarkeit der Patienten der beiden Gruppen gegenübergestellt.

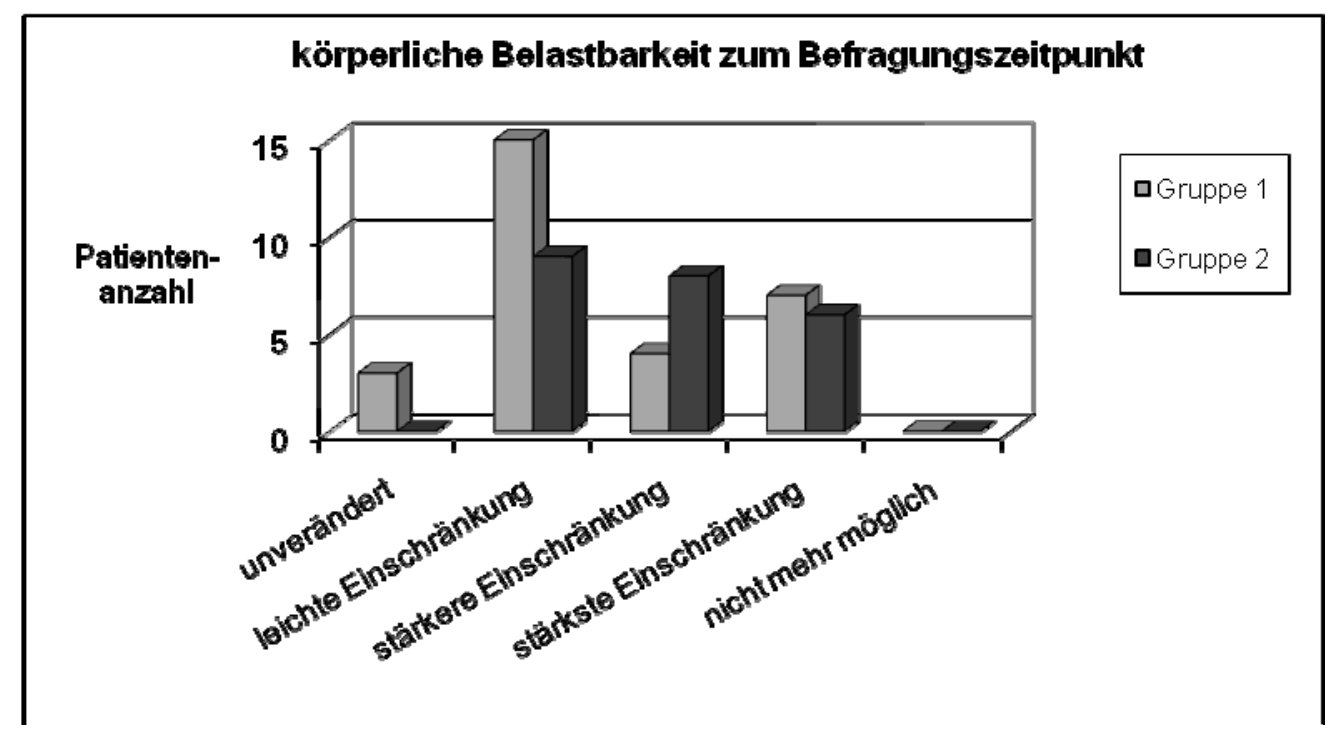

Abbildung 65: körperliche Belastbarkeit zum Befragungszeitpunkt in Gruppe 1 und 2.

\subsubsection{Auswertung der klinischen Nachuntersuchung}

24 Patienten der Gruppe 1 (82,76\%) und 20 Patienten (86,96\%) der Gruppe 2, die einen Fragebogen beantwortet haben, waren auch zu einer klinischen Untersuchung in der Universitätsmedizin Göttingen bereit. 6 der angeschriebenen Patienten (14,29\%) der Gruppe 1 und 7 Patienten (19,44\%) der Gruppe 2 lehnten sowohl die Beantwortung des Fragebogens als auch die klinische Untersuchung ab.

5 Patienten (17,24\%) der Gruppe 1 wünschten keine klinische Untersuchung, füllten aber einen Fragebogen aus. In Gruppe 2 waren dies 3 Patienten (13,04\%), die zwar einen Fragebogen ausfüllten, jedoch keine klinische Untersuchung wünschten.

\subsubsection{Altersverteilung zum Untersuchungszeitpunkt}

Zum Untersuchungszeitpunkt betrug das durchschnittliche Alter der klinisch nachuntersuchten Patienten der Gruppe 1 34,92 Jahre \pm 11,93 und der Gruppe 2 41,70 Jahre $\pm 17,04$. Die Gruppe 2 war von der Altersverteilung gering älter, jedoch zeigte sich mit $p=0,218$ kein signifikanter Unterschied zwischen den beiden Gruppen. 
In der nachfolgenden Abbildung ist die Altersverteilung zum Untersuchungszeitpunkt der beiden Gruppen nochmals gegenübergestellt.

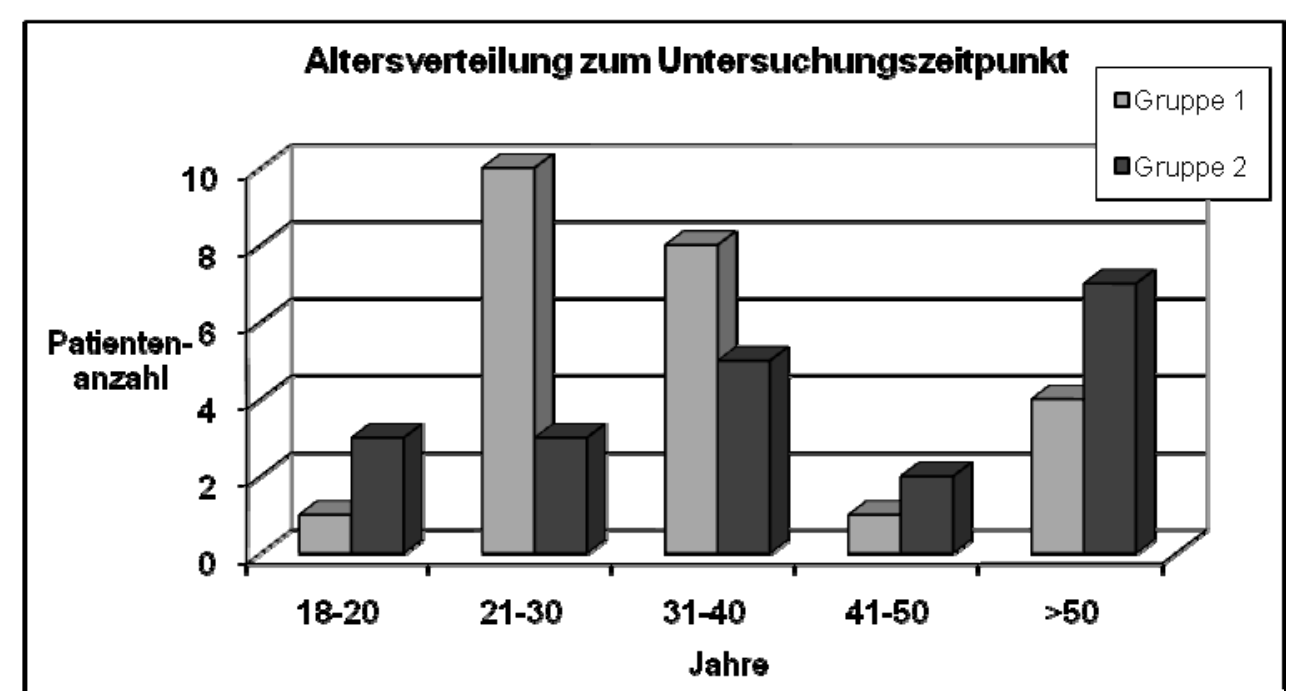

Abbildung 66: Altersverteilung der Patienten der Gruppe 1 und 2 zum Untersuchungszeitpunkt.

\subsubsection{Bodymassindex (BMI) der untersuchten Patienten}

Der Bodymassindex (BMI) wird aus dem Körpergewicht (KG in $\mathrm{kg}$ ) und der Körpergröße (in $\mathrm{m}$ ), wie folgt berechnet : $\quad \mathrm{BMI}=\mathrm{KG} /(\mathrm{Gr})^{2}$. Mit $\mathrm{p}=0,01$ zeigte sich in der Untersuchung der Gruppen ein signifikanter Unterschied, dahin gehend, dass in Gruppe 2 der BMl höher lag als in Gruppe 1, die Patienten der Gruppe 2 waren adipöser. Der BMI-Mittelwert der Gruppe 1 lag bei 23,67 $\pm 3,22$; in Gruppe 2 betrug er 27,16 \pm 3,82. In Gruppe 1 betrug das BMI-Minimum 16,65 und das BMI-Maximum 29,07; in Gruppe 2 betrug das BMI-Minimum 22,09 und das BMI-Maximum 34,72.

\subsubsection{Gangbild und Hilfsmittelnutzung}

54,17\% ( $N=13)$ der untersuchten Patienten der Gruppe 1 zeigten ein unauffälliges Gangbild, in der Gruppe 2 waren es nur 35,0\% (N=7) der Patienten. Ein Hinken bzw. unsicheres Gangbild zeigten 45,83\% der Gruppe-1-Patienten und 65,0\% der Gruppe2-Patienten. Ein signifikanter Unterschied zeigte sich jedoch hierbei nicht $(p=0,203)$. 
Hinsichtlich der Hilfsmittelnutzung wie Schuherhöhung, Gehstock etc. zeigte sich zwischen den beiden Gruppen mit $p=0,845$ ebenfalls kein signifikanter Unterschied. Mehrfachnennungen waren hierbei möglich. Die nachfolgende Abbildung zeigt die Hilfsmittelversorgung in beiden Gruppen.

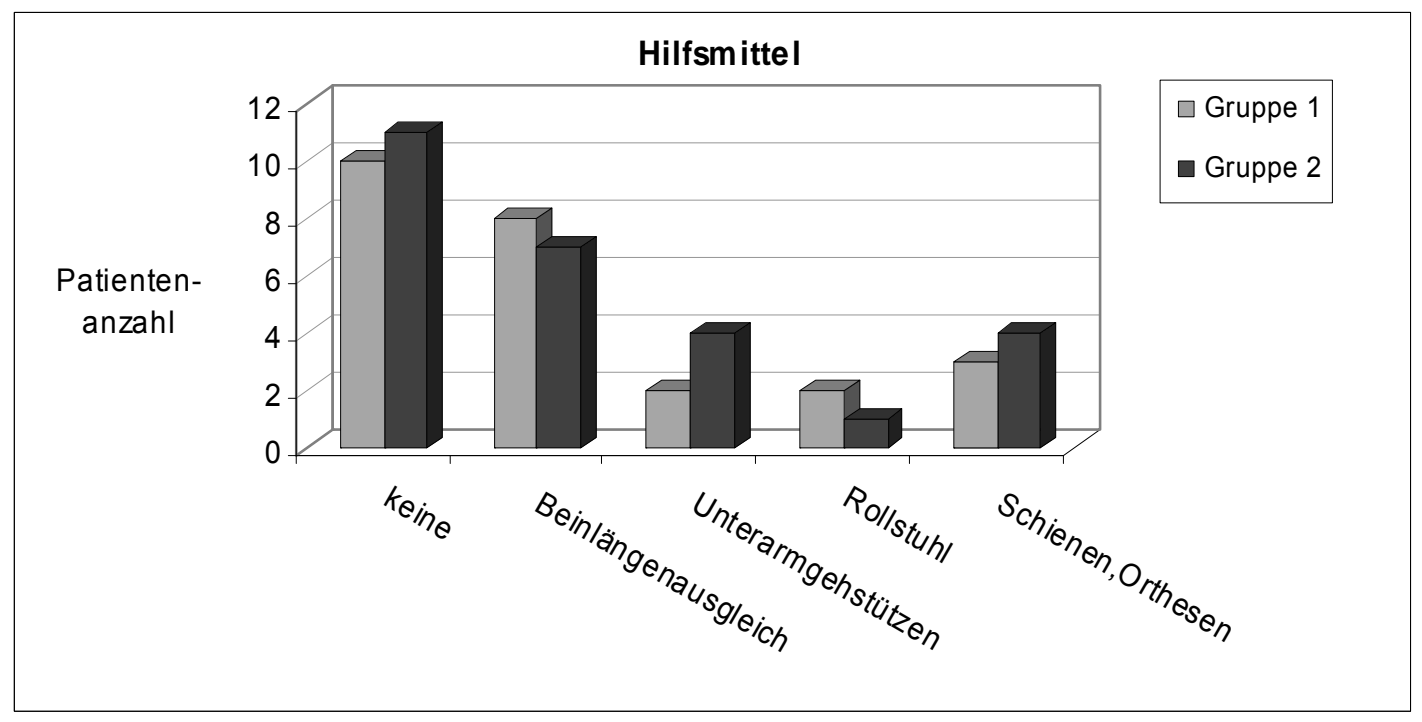

Abbildung 67: Hilfsmittelversorgung in Gruppe 1 und 2.

\subsubsection{Differenzierte Standarten}

Bei der klinischen Untersuchung der Patienten wurden verschiedene Standarten sowie Kniebeuge getestet. Es wurde hierbei differenziert in „möglich“, „eingeschränkt“ und „nicht möglich“. In den nachfolgenden Abbildungen sind die jeweiligen Ergebnisse der Gruppen zum Vergleich aufgeführt. Signifikante Unterschiede fanden sich hierbei jedoch nicht.
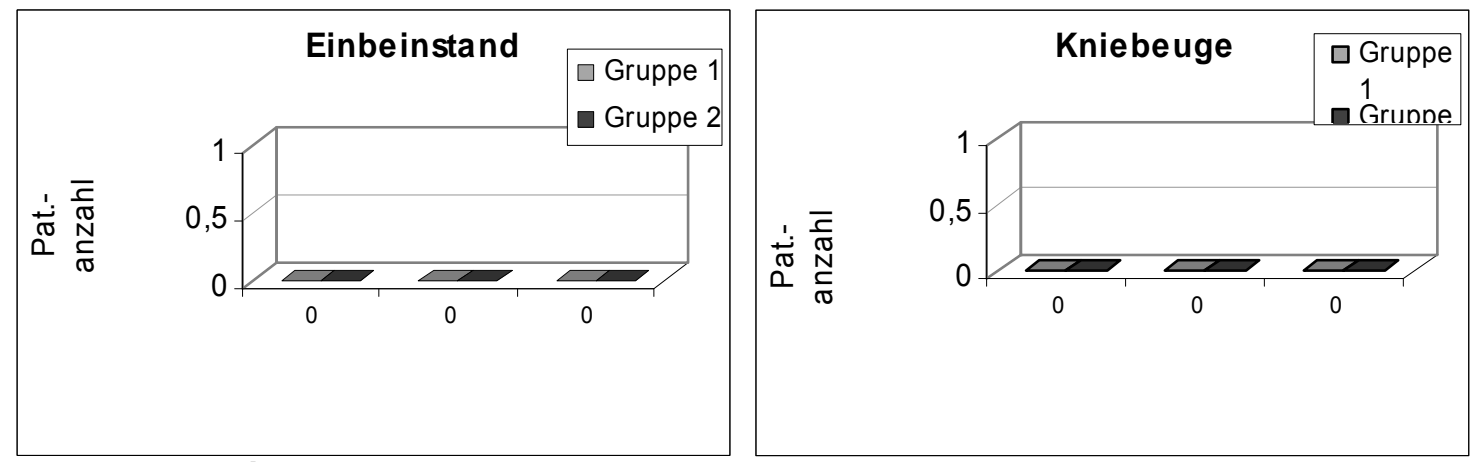

Abbildung 68/69: Ausführung des Einbeinstandes und der Kniebeuge in Gruppe 1 und 2. 


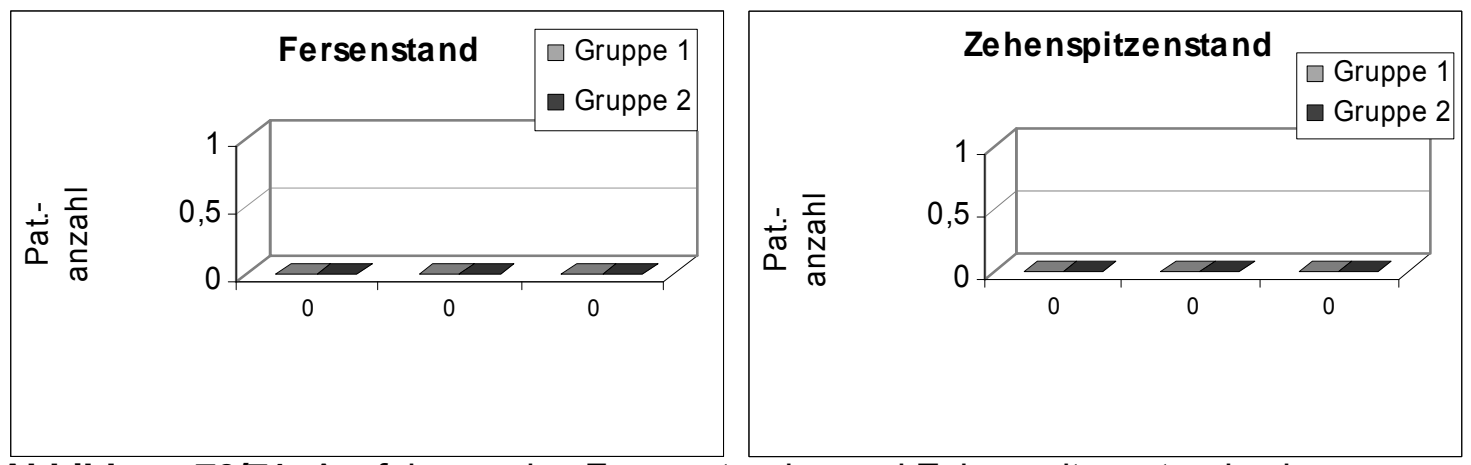

Abbildung 70/71: Ausführung des Fersenstandes und Zehenspitzenstandes in Gruppe 1 und 2.

Des weiteren wurde das Aufstehen aus dem Sitzen überprüft. Ohne zu Hilfenahme der Armlehnen konnten 87,5\% ( $\mathrm{N}=21)$ der Patienten der Gruppe 1 aufstehen, sowie 90,0\% ( $N=18)$ der Gruppe 2. Nur mit Hilfestellung bzw. unter zu Hilfenahme der Armlehnen konnten 12,5\% der Patienten der Gruppe 1 und 5,0\% der Gruppe 2 aufstehen.

\subsubsection{Schmerzlokalisation und Schmerzart}

Auf Befragung bei der klinischen Untersuchung gaben 70,83\% der untersuchten Patienten der Gruppe 1 an, dass sie Schmerzen haben. In der Gruppe 2 waren dies $85,0 \%$ der untersuchten Patienten. Bei der Schmerzlokalisation gaben 12 Patienten der Gruppe 1 unter anderem Schmerzen im Bereich der unteren Extremität an (mehrfach Nennungen waren hierbei möglich). In der Gruppe 2 waren dies 13 Patienten. Ein signifikanter Unterschied zwischen den beiden Gruppen zeigte sich nicht $(p=0,47)$. Die nachfolgende Abbildung gibt die differenzierte Verteilung der Schmerzlokalisation im Bereich der unteren Extremität wieder.

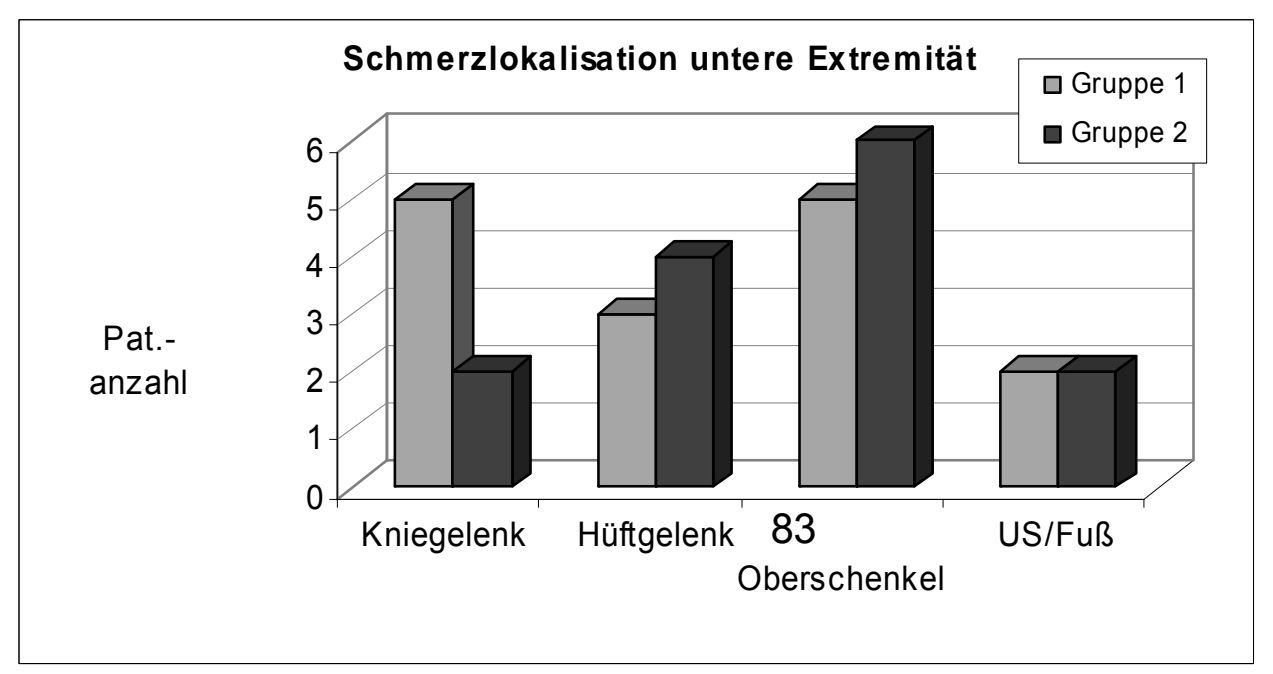


Abbildung 72: Schmerzlokalisation im Bereich der unteren Extremität in Gruppe 1 und 2.

Die nachfolgenden Abbildungen zeigen die verschieden Schmerzarten differenziert in den beiden zu untersuchenden Patientengruppen.

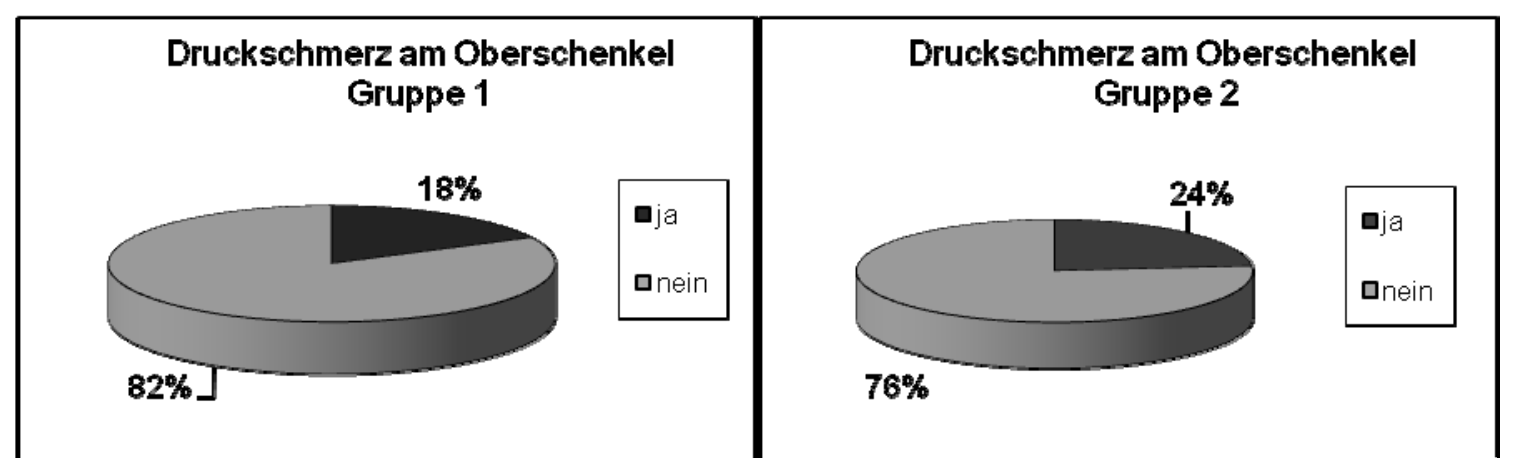

Abbildung 73/74: Druckschmerz am Oberschenkel in Gruppe 1 und 2.

(Ein signifikanter Unterschied zwischen den zwei Gruppen zeigte sich hierbei mit $p=0,67$ nicht.)
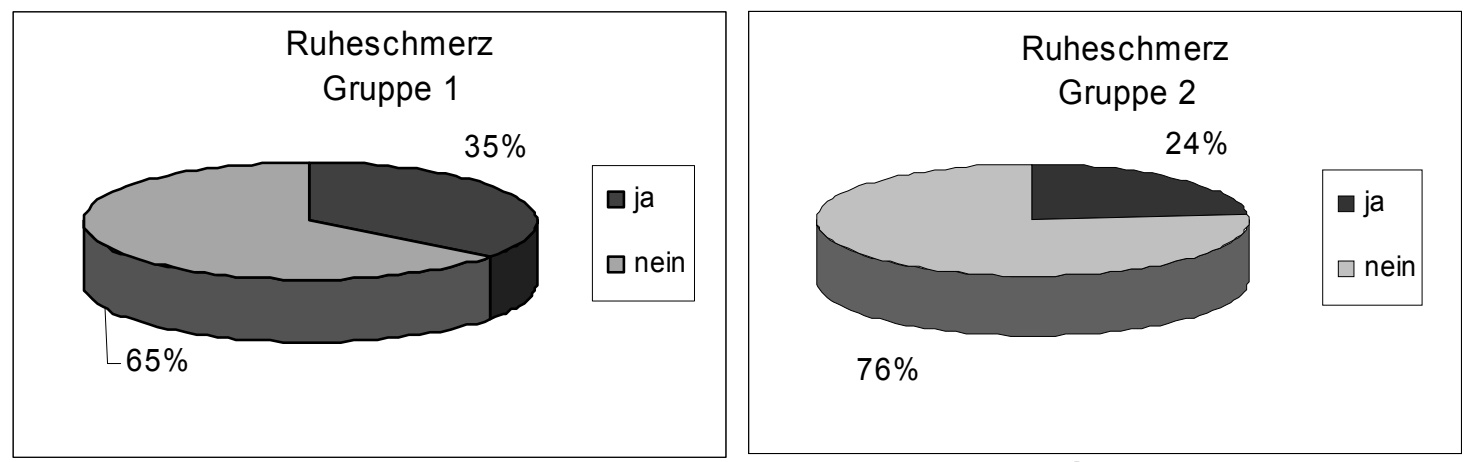

Oberschenkel in Gruppe 1 und 2.

Abbildung 75/76: Ruheschmerz am

(Ein signifikanter Unterschied zwischen den zwei Gruppen zeigte sich hierbei mit $p=0,45$ nicht.)

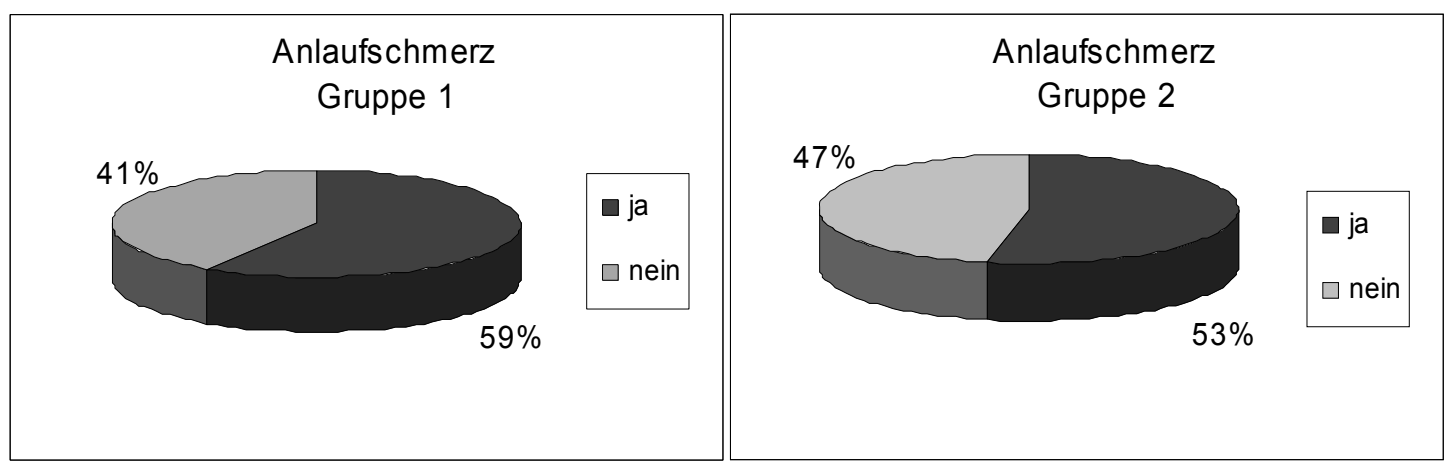

Abbildung 77/78: Anlaufschmerz im Bereich der Oberschenkels in Gruppe 1 und 2.

(Ein signifikanter Unterschied zwischen den zwei Gruppen zeigte sich hierbei mit $p=0,72$ nicht.) 


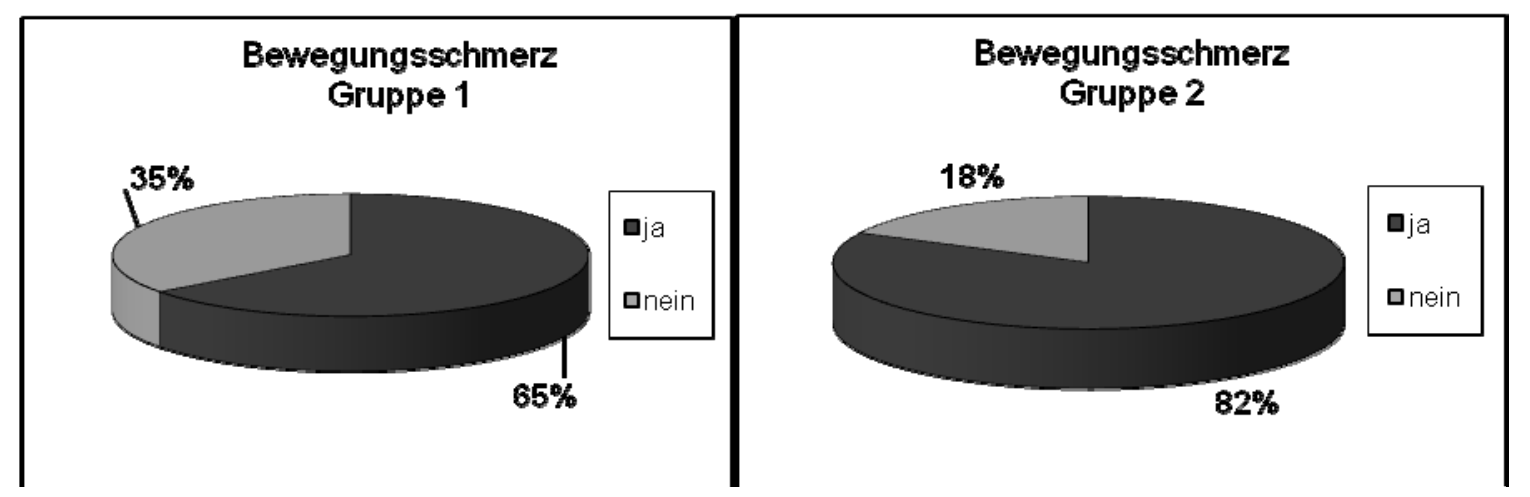

Abbildung 79/80: Bewegungsschmerz im Bereich der Oberschenkels in Gruppe 1 und 2. (Ein signifikanter Unterschied zwischen den zwei Gruppen zeigte sich mit $p=0,24$ nicht.)

\subsubsection{Längen- und Umfangsbestimmungen}

In dieser vorliegenden klinischen Untersuchung wurde die Beinlängendifferenz errechnet aus den jeweilig gemessenen Beinlängen der rechten und linken Seite (siehe Anhang nach Neutralnullmethode). Bei zwei Patienten der Gruppe 1 und einem Patienten der Gruppe 2 konnte keine Beinlängendifferenz errechnet werden, da auf einer Seite ein Zustand nach Amputation bestand. Der Mittelwert der Beinlängendifferenz der Gruppe 1 betrug $-0,295 \pm 0,701 \mathrm{~cm}$ und der Gruppe 2 $-0,342 \pm 0,727 \mathrm{~cm}$, ein signifikanter Unterschied $(p=0,86)$ zwischen den beide Gruppen zeigte sich nicht. In der nachfolgenden Abbildung sind die Beinlängendifferenzen der Patienten nochmals aufgeführt.

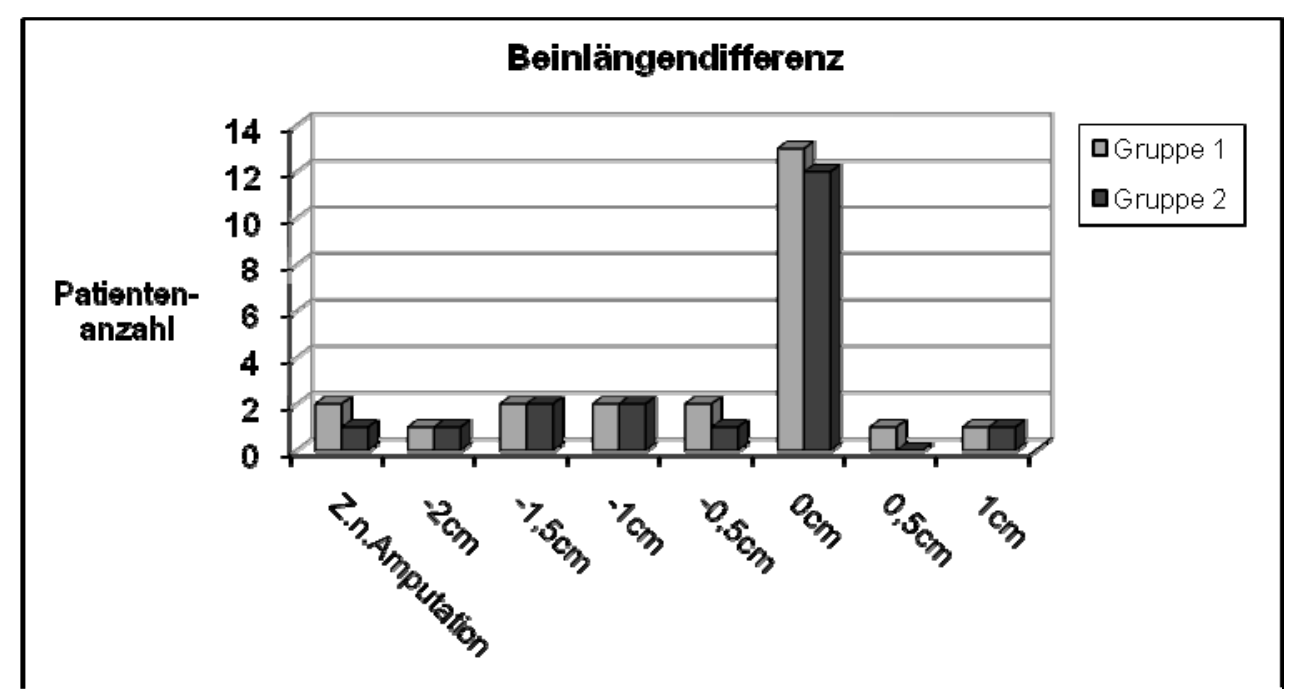

Abbildung 81: Beinlängendifferenz der betroffenen Seite in Gruppe 1 und 2. 
Bei der Berechnung der Oberschenkellängendifferenz betrug der Mittelwert der Gruppe $1-0,340 \pm 0,643 \mathrm{~cm}$ und der Gruppe $2-0,316 \pm 0,605 \mathrm{~cm}$, ein signifikanter Unterschied $(p=0,92)$ zeigte sich hierbei ebenfalls nicht. Die Verteilung auf die einzelnen berechneten Oberschenkellängendifferenzen zeigt die nachfolgende Abbildung.

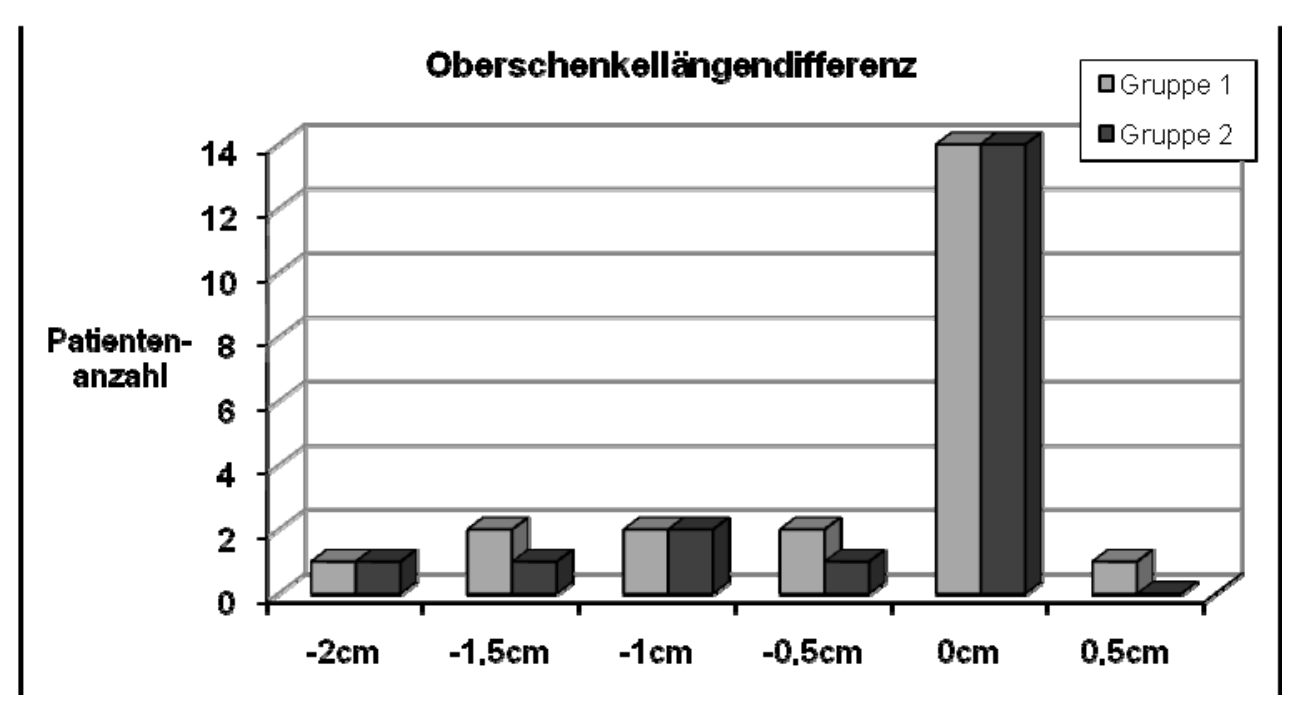

Abbildung 82: Oberschenkellängendifferenz in Bezug auf die betroffene Seite in Gruppe 1 und 2.

Der Mittelwert für die Oberschenkelumfangsdifferenz im Hinblick auf die betroffene Seite in Messhöhe 1 betrug in Gruppe $1-0,0681 \pm 2,867 \mathrm{~cm}$ und in Gruppe 2 $0,00 \pm 2,593 \mathrm{~cm}$. Ein signifikanter Unterschied zwischen den Gruppen zeigte sich nicht $(p=0,614)$.

Der Mittelwert für die Oberschenkelumfangsdifferenz in Messhöhe 2 betrug in Gruppe $1 \quad 0,3636 \pm 1,677 \mathrm{~cm}$ und in Gruppe $2 \quad-0,526 \pm 1,982 \mathrm{~cm}$. Auch hier zeigte sich zwischen den beiden Gruppen kein signifikanter Unterschied $(p=0,444)$. In den zwei nachfolgenden Abbildungen sind die Verteilungen der ermittelten Oberschenkelumfangsdifferenzen in Messhöhe 1 und 2 aufgeführt. 


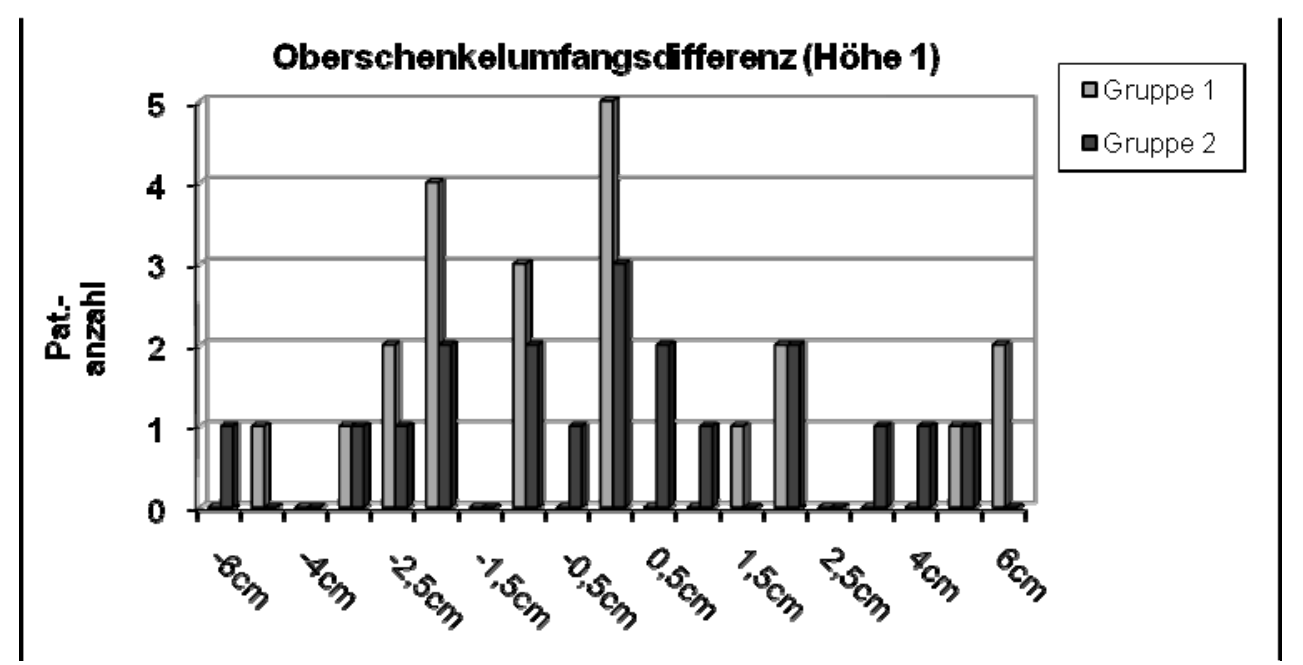

Abbildung 83: Oberschenkelumfangsdifferenz in Höhe1 gemessen in Gruppe 1 und 2.

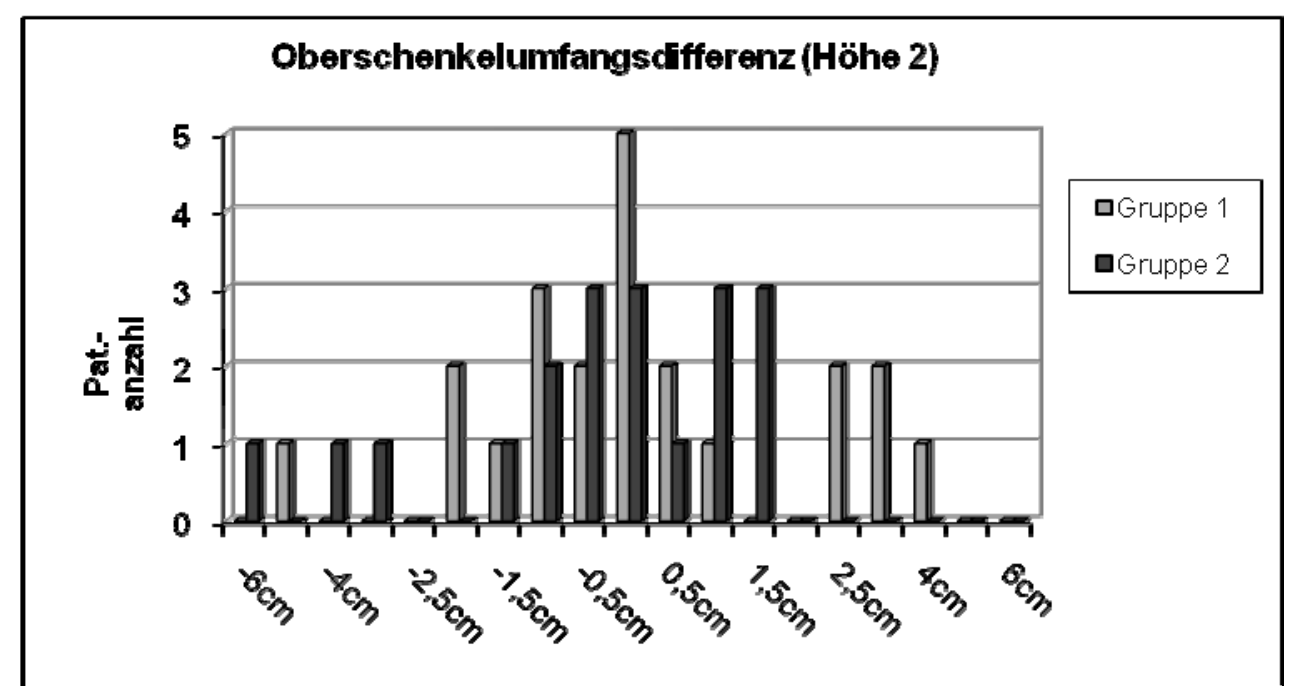

Abbildung 84: Oberschenkelumfangsdifferenz gemessen in Höhe 2 in Gruppe 1 und 2.

3.5.2.7. Bestimmung der Bewegungsausmaße der angrenzenden bzw. betroffenen Gelenke

In der vorliegenden Arbeit wurden bei der klinischen Nachuntersuchung die Bewegungsausmaße der angrenzenden bzw. betroffenen Gelenke (Hüftgelenk und Kniegelenk) nach der Neutral-Null-Methode bestimmt. In den zwei nachfolgenden Tabellen sind die Mittelwerte bzw. Standardabweichungen und errechneten Varianzen der jeweiligen Bewegungsrichtungen bzw. nach Gelenken unterschieden aufgeführt. 
Einen signifikanten Unterschied zwischen den zu untersuchenden Gruppen gab es im Hinblick auf die verschiedenen Bewegungsausmaße und Richtungen nicht.

Tabelle 13: Bewegungsausmaße Gruppe 1

\begin{tabular}{|l|c|c|c|c|c|c|c|}
\hline \hline Bewegung & N & Mittelwert & Median & Min & Max & Varianz & Standard- \\
\hline Hüftgelenk & 21 & 26,43 & 20,0 & 0 & 55,0 & 205,36 & 14,3303 \\
\hline Extension/Flexion & & & & & & & \\
\hline Aro/Iro bei Beugung & 20 & 16,25 & 15,0 & 0 & 40,0 & 204,93 & 13,3155 \\
\hline Aro/Iro bei Streckung & 18 & 13,05 & 12,5 & 0 & 30,0 & 103,35 & 10,1661 \\
\hline Abduktion/Adduktion & 21 & 2,38 & 0 & 0 & 10 & 19,04 & 4,3643 \\
\hline Kniegelenk & 21 & 8,80 & 0 & 0 & 60 & 247,26 & 15,7246 \\
\hline Extension/Flexion & & & & & & & \\
\hline
\end{tabular}

Tabelle 14: Bewegungsausmaße Gruppe 2

\begin{tabular}{|l|c|c|c|c|c|c|c|}
\hline Bewegung & N & Mittelwert & Median & Min & Max & Varianz & Standard- \\
\hline Hüftgelenk & 17 & 25,29 & 25,0 & 0 & 40,0 & 220,22 & 10,9645 \\
\hline Extension/Flexion & & & & & & & \\
\hline Aro/Iro bei Beugung & 17 & 19,71 & 20,0 & 0 & 50,0 & 188,57 & 13,7466 \\
\hline
\end{tabular}




\begin{tabular}{|l|c|c|c|c|c|c|c|}
\hline Aro/Iro bei Streckung & 16 & 13,13 & 10,0 & 0 & 60,0 & 206,25 & 14,3614 \\
\hline Abduktion/Adduktion & 17 & 3,82 & 0 & 0 & 30,0 & 61,03 & 7,8121 \\
\hline Kniegelenk & 17 & 8,52 & 0 & 0 & 80,0 & 393,02 & 18,8246 \\
\hline Extension/Flexion & & & & & & & \\
\hline
\end{tabular}

3.5.2.8. Modifizierter Score nach Neer et al. (1967)

Zum Abschluss der klinischen Untersuchung wurde ein in Anlehnung an den Untersuchungsscore nach NEER et al. (1967) eigens entwickeltes Beurteilungsschema verwendet, hierbei wurde mehr auf die klinischen und soziologischen Ergebnisse eingegangen und weniger auf etwaige röntgenologische Ergebnisse, da die Patienten beim Nachuntersuchungstermin nicht erneut geröntgt wurden. Der entwickelte Score befindet sich im Anhang.

Bei der Auswertung des erstellten Scores zeigte sich, dass bei $45,5 \%$ der nachuntersuchten Patienten der Gruppe 1 ein sehr gutes Ergebnis erzielt wurde, in Gruppe 2 nur bei 26,3\%. Im Vergleich der Gruppen konnte insgesamt jedoch kein signifikanter Unterschied $(p=0,509)$ gezeigt werden. Der Mittelwert der Gruppe 1 für den erstellten Score errechnete sich mit 60,45 \pm 18,27 Punkte, für Gruppe 2 betrug der Mittelwert 56,05 \pm 17,94 Punkte.

In der nachfolgenden Abbildung sind die verschiedenen Verteilungen der Punktwerte innerhalb der beiden Gruppen aufgeführt.

68 - 85 Punkte : sehr gutes Ergebnis

$47-67$ Punkte : gutes Ergebnis

22 - 46 Punkte : mäßiges Ergebnis

$0-21$ Punkte : nicht zufriedenstellendes Ergebnis 


\section{Ergebnisse}

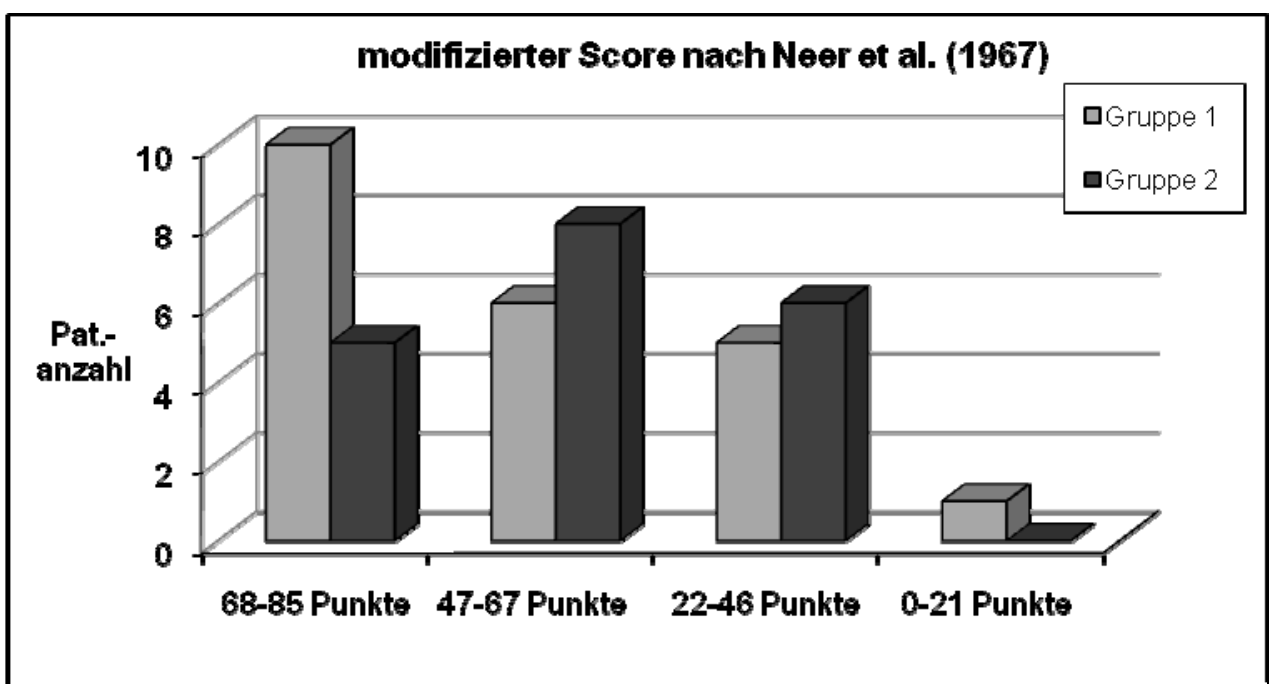

Abbildung 85: Auswertung des Beurteilungscores in Anlehnung an den Neer-Score in Gruppe 1 und 2. 


\section{Diskussion}

Obwohl primär-operative Versorgungsstrategien bei Verletzungen langer Röhrenknochen vor allem im Bereich der unteren Extremität im Rahmen eines Polytraumas eine zentrale klinische Rolle spielen, gibt es hierzu nur wenig Studienevidenz (RIXEN et al. 2005 a). Es wird, wie bereits in der Einleitung erläutert, nach zwei verschiedenen Konzepten verfahren, zum einen "Damage-control“ (DC)Konzept und das „Early-total-care“ (ETC)-Konzept. Bei dem DCO-Konzept (Damage Control Orthopaedics), dem DC-Konzept der Unfallchirurgie, geht es um die primäre schnelle Stabilisierung von Frakturen mittels Fixateur extern mit entsprechender Weichteilversorgung, um den Patienten für die intensivpflegerischen Maßnahmen lagerungsstabil herzurichten. Dabei geht es vor allem um eine signifikante Reduzierung der Operationszeiten sowie Reduktion des Blutverlustes in der Primärphase (TAEGER et al. 2005, TUTTLE et al. 2009). Im Gegensatz dazu steht seit den 1980er Jahren das ETC-Konzept, welches eine definitive Versorgung von Extremitätenverletzungen in der Primärphase fordert, Ziel ist hierbei eine frühe Mobilisierung und Schmerzreduktion zu erreichen, insbesondere auch bei Patienten mit zunehmender Verletzungsschwere (BONE et al. 1989). Allerdings gab es hierzu auch gegenteilige Untersuchungsergebnisse, welche zeigten, dass es durch primäre Nagelung zu einem zusätzlichen Schaden durch Länge der Operationszeit und eine deutliche inflammatorische Antwort, sowie erhöhte Raten an ARDS kommt (PAPE et al. 2002 und 2004, PAPE und KRETTEK 2003).

RIXEN et al. (2005 a) haben durch eine systematische Literaturrecherche folgende Punkte zur Versorgung langer Röhrenknochen beim Polytrauma herausgearbeitet:

- Proximale Femurfrakturen sollten im Sinne des ETC in der Primärphase osteosynthetisch versorgt werden, als DC-Konzept käme ein gelenkübergreifender Fixateur extern in Frage.

- Isolierte und multiple Schaftfrakturen sollten in der Primärphase mittels Verriegelungsmarknagelung versorgt werden, ein abgestuftes Konzept wäre die Versorgung mittels Fixateur extern.

- Distale Femurfrakturen sollten frühzeitig temporär stabilisiert werden und im Verlauf dann sekundär rekonstruiert werden.

Im Untersuchungsgut der hier vorliegenden Arbeit wurde primär-operativ die vorhandene Femurfraktur zu 26,5\% mit einem Fixateur extern versorgt, was dem Konzept des DC entspricht. Weitere Versorgungen erfolgten mit $19,4 \%$ durch 
Marknagelung und mit 25,5\% durch Plattenosteosynthese, eine Extension erhielten 5,1\% der Patienten mit Femurfraktur. Weitere Osteosynthesearten wie Kondylenplatte, Dynamische Hüftschraube, Winkelplatte wurden zu 21,3\% durchgeführt. In 2,04\% der Fälle musste eine Amputation im Oberschenkelbereich erfolgen.

Es zeigten sich beim Vergleich der Patienten, die mit einem Fixateur extern versorgt wurden, und solche die primär mit eine Marknagel oder einer Platte osteosynthetisch am Femur versorgt wurden, signifikante Unterschiede hinsichtlich des ISS, der Intensivaufenthaltsdauer und der Beatmungsdauer. Bei den Patienten, die mittels Fixateur versorgt wurden, zeigte sich ein deutlich höherer ISS, was auf eine höhere Verletzungsschwere hinweist, sowie verlängerte Intensivaufenthaltsdauer und Beatmungsdauer. Gleiche Ergebnisse fanden auch MOMMSEN et al. (2009) in ihrer retrospektiven Studie im Vergleich der Versorgung von Femurschaftfrakturen nach DCO-Prinzip und ETC-Prinzip.

Die unterschiedlichen Entscheidungen zu den jeweiligen operativen Versorgungen der Femurfraktur beim Polytrauma zeigen sich auch in der Datenlage des DGUTraumaregisters. In einigen Kliniken wird primär mit einem Fixateur extern und in anderen Kliniken wiederum mit einem Marknagel primär versorgt (RIXEN et al. 2005 a). Es erfolgt in dieser Arbeit ein Vergleich mit den vorhandenen Daten aus dem DGU-Traumaregister-Jahresbericht 2000 (www.traumaregister.de (2000)).

In der erstellten Arbeit zeigt sich für die Polytraumapatienten mit Femurfraktur ein Altersdurchschnitt von 34,95 Jahren, die Patienten sind im Vergleich zum Durchschnittsalter des DGU-Jahresberichtes 2000 (www.traumaregister.de (2000)) mit 40,6 Jahren etwas jünger. Bei der Geschlechtsverteilung überwiegt wie auch in den Zahlen der DGU 2000 ein männlicher Anteil an den Verletzten mit 70\%.

Eine primäre Versorgung in der Universitätsmedizin Göttingen erfolgte bei 78,65\% der Polytraumapatienten mit Femurfraktur, laut den Ergebnissen der DGU wurden im Jahr 2000 ebenfalls 78,1\% in der Primärklinik versorgt.

Der errechnete ISS-Mittelwert im DGU-Jahresbericht 2000 (www.traumaregister.de (2000)) betrug 23,8, im Vergleich betrug der ISS-Mittelwert in der hier vorliegenden Arbeit bei den Polytraumapatienten mit Femurfraktur 28,38 und war somit etwas höher ausgefallen, die Verunfallten waren von der Verletzungsschwere höher einzustufen. Beim Vergleich der beiden Gruppen mit Femurfraktur zeigte sich ein mit 29,29 etwas 
höherer ISS-Mittelwert in Gruppe 1 als in Gruppe 2 mit 27,18, ein signifikanter Unterschied konnte jedoch nicht nachgewiesen werden $(p=0,417)$.

Die Mortalität betrug im DGU-Jahresbericht 2000 (www.traumaregister.de (2000)) 16,7\%. Im Vergleich hierzu betrug sie bei den im Rahmen dieser Arbeit untersuchten Patienten 12,1\%, obwohl laut ISS-Mittelwert eher aufgrund der höheren Verletzungsschwere mit einem höheren Prozentanteil zurechnen war.

Beim Vergleich der Intensivaufenthaltsdauer zeigte sich in unserem untersuchten Patientengut mit Femurfraktur in der Abteilung für Unfallchirurgie der Universitätsmedizin Göttingen ein etwas verlängerter Aufenthalt mit 15,35 Tagen, bei der DGU betrug sie 11,0 Tage. Auch bei der Beatmungsdauer zeigte sich eine etwas verlängerte Dauer mit 7,13 Tagen im Vergleich zur DGU mit 6,5 Tagen. Insgesamt decken sich aber die Daten unseres Polytraumaregisters mit denen des DGUJahresberichtes 2000 (www.traumaregister.de (2000)).

Eine verlängerte Dauer zeigte sich für den gesamtstationären Aufenthalt der Polytraumapatienten mit Femurfraktur in der Universitätsmedizin Göttingen mit einem Mittelwert von 47,33 Tagen, im Vergleich hierzu betrug die stationäre Aufenthaltsdauer des DGU-Traumaregisters 2000 (www.traumaregister.de (2000)) nur 31,7 Tage. Es ist hierbei zu beachten, dass die Verletzungsschwere im Göttinger Patientengut im Vergleich zur DGU 2000 etwas höher lag und darin auch eine mögliche Ursache für einen verlängerten Aufenthalt zu sehen ist. Laut DGU-Jahresbericht 2000 (www.traumaregister.de (2000)) wurden 17,7\% der verunfallten und überlebenden Patienten in ein anderes Krankenhaus verlegt, 25,3\% wurde in eine REHA und 32,5\% direkt nach Hause entlassen. Bei den hier in der Arbeit erfassten Daten wurden 47,5\% der Patienten mit Femurfraktur in eine REHA entlassen, weitere 12,5\% in ein anderes Krankenhaus verlegt und $17,5 \%$ direkt nach Hause entlassen.

In der nachfolgenden Tabelle sind die prozentualen Anteile der verschiedenen Verletzungsursachen der DGU 2000 und der Polytraumapatienten mit Femurfraktur aus der Universitätsmedizin Göttingen gegenüber gestellt. Es zeigt sich hier ein höherer Anteil an Verkehrsunfällen als Verletzungsursache im Vergleich zu den Angaben der DGU 2000, während der Anteil an sonstigen Unfallursachen oder Suizid in der hier untersuchten Gruppe der Polytraumatisierten mit Femurfraktur deutlich geringer ist. 
Tabelle 15: Unfallursachen im DGU-Jahresbericht im Vergleich zur hier vorliegenden Arbeit.

\begin{tabular}{|l|c|c|}
\hline & $\begin{array}{c}\text { DGU -Jahresbericht 2000 } \\
\text { (www.traumaregister.de (2000) }) \\
\%(\mathrm{~N})\end{array}$ & $\begin{array}{c}\text { Polytrauma mit } \\
\text { Femurfraktur d. } \\
\text { Universitätsmedizin } \\
\text { Göttingen } \\
\%(\mathrm{~N})\end{array}$ \\
\hline Verkehrsunfall & $60,5 \%(1468)$ & $92,3 \%(84)$ \\
\hline => PKW / LWK-Insasse & $59,3 \%(871)$ & $73,8 \%(62)$ \\
\hline => Zweirad & $28,2 \%(414)$ & $21,4 \%(18)$ \\
\hline => Fußgänger & $12,5 \%(183)$ & $4,8 \%(4)$ \\
\hline Absturz aus > 3 m Höhe & $11,1 \%(270)$ & $4,4 \%(4)$ \\
\hline Sonstiges (inkl. Suizid) & $25,9 \%(627)$ & $3,3 \%(3)$ \\
\hline
\end{tabular}

In der Literatur findet man variierende Angaben über den prozentualen Anteil der Extremitätenverletzung am Verletzungsmuster, so zeigte sich in einer Datenerhebung von WENINGER et al. (2005) ein Anteil von 68,3\% mit Frakturen der langen Röhrenknochen beim Polytrauma, am häufigsten seien hierbei Femurfrakturen mit 73,2\% vorhanden (WENINGER et al. 2005). Mit 69\% Extremitätenverletzung gaben auch TRUPKA et al. (1995), PIEK et al. (2000) und WALCHER (2003) den Anteil am Polytrauma an. In einer weiteren Studie von RUCHHOLTZ et al. (1996) zeigten sich abhängig vom Unfallmechanismus unterschiedliche Anteile, so sei der Prozentsatz von Verletzungen der unteren Extremität bei Motorradfahrern sogar 90\% und hierbei der Anteil von Femurfrakturen bis zu 85\%, wohingegen bei PKW-Frontalunfällen mit Gurt nur in $25 \%$ eine Extremitätenverletzung vorlag. Bei dem Gesamtkollektiv traten die Verletzungen an der unteren Extremität mit 56\% am zweithäufigsten nach den Thoraxverletzungen auf (RUCHHOLTZ et al. 1996). In der hier vorliegenden Arbeit zeigte sich im Untersuchungszeitraum von 1994 bis 2000 im Patientengut der Universitätsmedizin Göttingen ein Anteil von 64,9\% an Extremitäten/

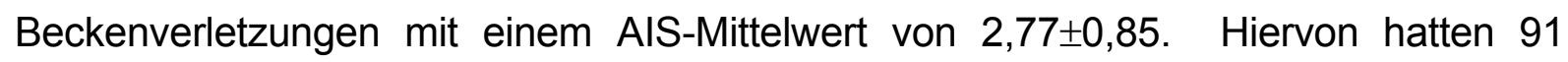
Patienten (20,5\%) eine Femurfraktur, wobei bei 10 Patienten eine beidseitige Fraktur 
vorlag. Hinsichtlich der Unfallursache zeigte sich, dass 68\% als PKW-Insassen und $21,4 \%$ als Zweiradfahrer verunfallt waren.

In der aktuellen Literatur gibt es derzeit keine Angaben, darüber welche Seite bei Femurfrakturen gehäuft verletzt wird. Der in dieser Arbeit gezeigte, deutlich höhere Anteil der linken Seite mit 62,64\% der Fälle im Vergleich zur rechten Seite mit $26,37 \%$, kann deshalb nicht für allgemein gültig erklärt werden.

In unserem Patientenkollektiv wurde bei $28,57 \%$ der Patienten eine offene Femurfraktur dokumentiert. Hierbei handelte es sich bei $46,2 \%$ um erstgradig offene Frakturen und jeweils bei 26,9 \% um zweit- und drittgradig offene Frakturen. In der Studie um WENINGER et al. (2005) zeigte sich zum Vergleich ein Anteil von $71,8 \%$ an offenen Frakturen der langen Röhrenknochen mit einem hohen Anteil von 59,3\% an höhergradigen offenen Frakturen.

Beim Vergleich der beiden Gruppen (wie im Methodenteil bereits beschrieben) zeigte sich ein signifikanter Unterschied mit $p=0,002$ hinsichtlich der primären Krankenhausversorgung. So wurden 90,38\% der Patienten der Gruppe 1 primär über den Schockraum der Universitätsmedizin Göttingen eingeliefert, während nur 62,16\% der Gruppe 2 primär in der Universitätsmedizin Göttingen behandelt wurden und $37,84 \%$ sekundär erst nach externer Primärversorgung nach Göttingen verlegt worden sind.

Ein weiterer signifikanter Unterschied zeigte sich beim Vergleich der beiden Gruppen hinsichtlich der Unfallursache, so waren in der Gruppe 1 deutlich mehr Personen $(21,2 \%)$ mit einem Zweirad verunfallt als in Gruppe 2.

Die Altersstruktur der beiden Gruppen zeigte keinen signifikanten Unterschied mit

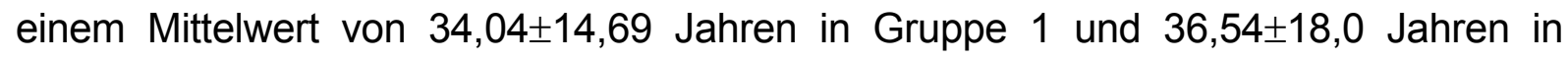
Gruppe 2. Dies entspricht auch annähernd der Altersstruktur aus der Gesamtpolytraumastudie über den benannten Zeitraum mit einem Mittelwert von $39,7 \pm 21,0$ Jahren. Auch hinsichtlich der Geschlechtsverteilung zeigten sich zwischen den beiden Gruppen keine signifikanten Unterschiede.

Die Bestimmung des Hämoglobin- wie auch des Hämatokritwertes gehören It. FRANK et al. (1996) zu den wichtigen Maßnahmen zum Monitoring und der 
Labordiagnostik bei Aufnahme und im weiteren Verlauf eines Polytraumapatienten. In der hier vorliegenden Arbeit lagen bei beiden Gruppen die Hämoglobin- und Hämatokritwerte bei Aufnahme jeweils deutlich unter den Referenzwerten. Es zeigte sich des weiteren, dass die im weiteren Verlauf verstorbenen Patienten einen noch deutlicher erniedrigten Hämoglobin- und Hämatokritwert aufwiesen. In diesem Zusammenhang berichtet HUTSCHENREUTER (2000), dass diese beiden Werte wichtige Indikatoren für Blutverluste seien, welche zu einem hämorrhagischen Schock (ZIEGENFUß 1996) mit den entsprechenden Folgen führen kann. Hierzu ist anzumerken, dass es bei Frakturen großer Röhrenknochen auch zu entsprechenden Blutverlusten von 1000-3000ml kommen kann (RASCHKE und HAAS 1997).

Bei dem Vergleich der Einteilung der Frakturen nach der AO-Klassifikation zeigte sich zwischen den beiden Gruppen ebenfalls kein wesentlicher Unterschied, was natürlich die Gruppen auch im Hinblick auf die operative Versorgung besser vergleichbar macht. Bei der Auswertung der operativen Primärversorgung der Femurfrakturen zeigte sich, dass in Gruppe 1 deutlich mehr Patienten (37,93\%) mit einem Fixateur extern versorgt wurden als in Gruppe 2 mit lediglich 9,3\%. In Gruppe 2 wurden deutlich mehr Patienten $(30,2 \%)$ mit einem Marknagel als in Gruppe $2(11,1 \%)$ versorgt. Der Anteil an plattenosteosynthetischer Versorgung war in beiden Gruppen annähernd gleich mit $25,9 \%$ in Gruppe 1 und 25,6\% in Gruppe 2. Bei 27,6\% der Patienten der Gruppe 1 wurde ein Verfahrenswechsel durchgeführt, dagegen in Gruppe 2 nur bei 11,6\%. Dies ist durch den höheren Anteil von primär mit Fixateur extern versorgten Patienten der Gruppe 1 zu erklären. 36\% der Patienten der Gruppe 1 die einen Verfahrenswechsel erhielten wurden mit einem Marknagel versorgt, weitere 16\% mit einer Plattenosteosynthese. In Gruppe 2 waren der Anteil an Verfahrenswechsel auf einen Marknagel oder eine Plattenosteosynthese gleich mit 33\%.

Beim Vergleich der Begleitverletzungen zeigt sich in Gruppe 2 der größte Anteil von $51,3 \%$ mit einer Kombination von Verletzungen des Schädels sowie des Thorax. In Gruppe 1 beträgt der Anteil an zusätzlichen Kopf/Thorax-Verletzungen 28,8\%. Alleinige Kopfverletzung als Kombination zur Extremitätenverletzung kam in Gruppe 1 bei $26,9 \%$ und in Gruppe 2 bei $20,5 \%$ vor. Bei den Begleitverletzungen Thorax/Abdomen war der prozentuale Anteil in beiden Gruppen annähernd gleich mit 11,5\% in Gruppe 1 und 10,3\% in Gruppe 2. Ein signifikanter Unterschied zwischen den beiden Gruppen zeigte sich mit $p=0,16$ nicht. 
Bei der Beurteilung des Schweregrades des Verletzungsmusters ergab sich für

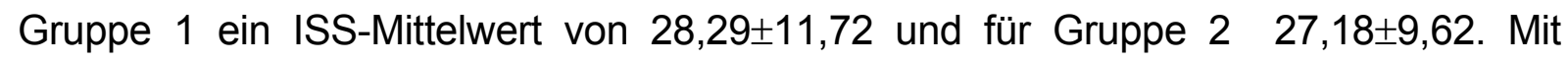
$p=0,42$ zeigte sich zwischen beiden Gruppe kein signifikanter Unterschied.

Hinsichtlich der Intensivstationären Aufenthaltsdauer und der Beatmungsdauer zeigten sich keine signifikanten Unterschiede zwischen den beiden untersuchten Gruppen. Der

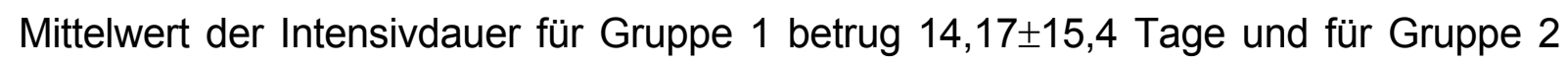
16,6 $\pm 19,52$ Tage $(p=0,86)$. Der Mittelwert für die Beatmungsdauer betrug in Gruppe 1 $7,24 \pm 8,19$ Tage und in Gruppe $27 \pm 8,17$ Tage $(p=0,48)$.

Auch bei der Gesamtstationären Aufenthaltsdauer konnte kein signifikanter Unterschied zwischen den Gruppen nachgewiesen werden, der Mittelwert der Gruppe

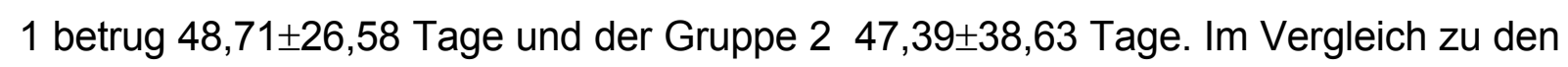
Daten des DGU-Jahresberichtes 2000 (www.traumaregister.de (2000)) ist die gesamtstationäre Aufenthaltsdauer in Göttingen etwas verlängert, was jedoch durch den auch höheren ISS im Patientengut der Universitätsmedizin Göttingen zu erklären ist.

In Gruppe 1 traten signifikant mit $p=0,039$ häufiger Komplikationen in 40,38\% der Fälle auf, in Gruppe 2 bei 38,46\%. 23,8\% der Komplikationen der Gruppe 1 waren pulmonale Komplikationen (zum Beispiel Pneumonie, Lungenembolie), in Gruppe 2 waren die vorherrschenden Komplikationen mit $20 \%$ jeweils Infektionen und das Multiorganversagen.

Zur Auswertung des bei der Nachuntersuchung erhobenen Fragebogens :

69,05\% der angeschriebenen Patienten der Gruppe 1 und 63,89\% der Gruppe 2 beantworteten den im Anhang befindlichen Fragebogen, damit lagen wir im Literaturvergleich im oberen Mittelfeld, WIEKEN (1974) gibt bei postalischer Befragung eine Rücklaufquote zwischen $10 \%$ und $90 \%$ an. Signifikant $(p=0,04)$ negativer wurde die sportliche Aktivität nach dem Trauma im Vergleich zu vorher in Gruppe 2 angegeben, so gaben 47,8\% der Befragten der Gruppe 2 an, ihren zuvor ausgeübten Sport nicht mehr durchführen zu können. Im Vergleich hierzu gaben 37,9\% der Gruppe 1 an, den selben Sport wie vor dem Unfall weiter durchführen zu können.

Für das funktionale und psychosoziale Outcome ist nach BOUILLON et al. (1998) bei $70 \%$ der Patienten mit einer Verschlechterung im Vergleich zum Zeitpunkt vor dem 
Unfallereignis zu rechnen. Im Hinblick auf die nach dem Trauma bestehende körperliche Belastbarkeit gaben 51,7\% der Gruppe 1 und 39,1\% der Gruppe 2 eine leichte Einschränkung an. Stärkere Einschränkungen der körperlichen Belastbarkeit gaben 13,8\% der Gruppe 1 und 34,8\% der Gruppe 2 an, stärkste Einschränkungen gaben in beiden Gruppen annähernd ein Viertel der Patienten an. Auch im Hinblick auf die Beantwortung nach der Gehstrecke konnte kein signifikanter Unterschied gezeigt werden.

Hinsichtlich soziodemographischer Daten aus der Fragebogenbeantwortung zeigte sich, dass in Gruppe 1 signifikant $(p=0,04)$ mehr Patienten $(48,3 \%)$ alleinstehend lebten als in Gruppe 2, wo die größere Anzahl mit 60,8\% verheiratet war. Bei den weiteren erhobenen soziodemographischen Daten wie Schulbildung, Berufsausbildung und weiteres zeigten sich keine signifikanten Unterschiede zwischen den beiden zu untersuchenden Gruppen. 62,1\% der befragten Patienten der Gruppe 1 gaben zum Befragungszeitpunkt an, dass sie berufstätig sind, während in Gruppe 2 nur 39,1\% zu diesem Zeitpunkt berufstätig waren, jedoch zeigte sich mit $p=0,1$ keine Signifikanz hierfür. $27,3 \%$ der nicht Berufstätigen der Gruppe 1 führten gerade eine Umschulung durch, in Gruppe 2 waren dies 21,4\%. Laut statistischem Bundesamt betrugen die volkswirtschaftlichen Kosten für Schwerverletzte im Jahr 2000 8,5 Mrd. Euro. In einer Nachuntersuchung von PIRENTE et al. (2001) waren es $14 \%$ der Schwerverletzten, die eine Umschulung nach dem Unfall durchführen mussten. Eine Erwerbsunfähigkeitsrente erhielten 27,3\% der Gruppe 1 und 35,7\% der Gruppe 2. Eine Arbeitsunfähigkeit nach dem Trauma von mehr als 12 Monaten gaben 55,2\% der Befragten der Gruppe 1 an und sogar 78,3\% der Befragten der Gruppe 2. Laut Daten des statistischen Bundesamtes Deutschland wurde im Jahr 2003 netto 1,3 Mrd. Euro für die Grundsicherung im Alter und bei Erwerbsminderung ausgegeben (Statistisches Bundesamt Deutschland (Abrufdatum 10.08.2009)). Dies belegt die erheblichen wirtschaftlichen Auswirkungen durch schwere Unfälle mit polytraumatischer Verletzung und wie wichtig es ist, eine entsprechende optimale Behandlung zu erreichen.

Noch bestehende Schmerzen nach dem Trauma gaben zum Befragungszeitpunkt 72,4\% der Patienten der Gruppe 1 und 78,3\% der Gruppe 2 an, wobei das Auftreten des Schmerzes bei 57,1\% der Gruppe 1 und 50\% der Gruppe 2 als gelegentlich eingestuft wurde. Nur 38,1\% der Patienten mit Schmerz der Gruppe 1 benötigen eine 
medikamentöse Schmerztherapie, in der Gruppe 2 waren es etwas mehr mit $50 \%$. Eine Signifikanz war nicht nachweisbar.

Hinsichtlich der Schmerzlokalisation betrug der Anteil der Patienten mit Schmerzen im betroffenen Oberschenkelbereich 25\%, im Hüftgelenk 5,6\% und Kniegelenk 11,1\% in Gruppe 1 und in Gruppe 2 im Oberschenkelbereich 8,7\%, im Hüftgelenk 21,7\% und Kniegelenk 13\%. Es zeigt sich hierbei, dass in Gruppe 1 mehr Patienten noch Schmerzen im Oberschenkelbereich haben, dagegen haben mehr Patienten der Gruppe 2 Schmerzen im Hüftgelenk, jedoch zeigte sich keine Signifikanz.

Bei der klinischen Nachuntersuchung fand sich im Vergleich der beiden zu untersuchenden Gruppen, dass in Gruppe 2 mit 65\% signifikant mehr adipöse Patienten waren als in Gruppe 1 mit 33,3\%. In der zur Verfügung stehenden Literatur fanden sich keine Untersuchungsdaten.

45,8\% der klinisch nachuntersuchten Patienten der Gruppe 1 zeigte ein hinkendes Gangbild, in Gruppe 2 waren dies sogar 65\%, jedoch ohne Nachweis einer Signifikanz.

Hinsichtlich der bis zum Nachuntersuchungszeitpunkt erfolgten Hilfsmittelversorgung zeigte sich kein signifikanter Unterschied zwischen den beiden Gruppen, so nutzten 8,3\% der Gruppe 1 und 20\% der Gruppe 2 noch Unterarmgehstützen auf längeren Strecken, des weiteren waren 8,3\% der Gruppe 1 und 5\% der Gruppe 2 mit einem Rollstuhl versorgt. Positiv ist anzumerken, dass 41,7\% der Patienten der Gruppe 1 und 55\% der Patienten der Gruppe 2 keinerlei Hilfsmittel mehr benötigten.

Bei der Untersuchung der verschiedenen Gang- und Standarten zeigten sich zwischen den zwei nachuntersuchten Gruppen keine signifikanten Unterschiede.

Bei der klinischen Untersuchung gaben 70,8\% der nachuntersuchten Patienten der Gruppe 1 und mit 85\% deutlich mehr der Gruppe 2 noch Schmerzen an, davon waren $54,5 \%$ und $65 \%$ jeweils im Bereich der unteren Extremität lokalisiert. Die Schmerzen äußerten sich vor allem unter Belastung mit 55\% in Gruppe 1 und deutlich mehr mit $82,4 \%$ in Gruppe 2. Eine Signifikanz hierfür war nicht nachweisbar.

Die Beinlängenmessung ergab bei $40,9 \%$ der nachuntersuchten Patienten der Gruppe 1 eine Beinlängendifferenz $\geq 0,5 \mathrm{~cm}$, in der Gruppe 2 bei 36,8\%. Im Vergleich hierzu gaben SCHMIDTMANN et al. (1997) in ihrer Studie über die plattenosteo- 
synthetische Versorgung bei 13 nachuntersuchten Patienten lediglich bei einem Patienten eine Beinlängendifferenz von $1,5 \mathrm{~cm}$ an. In einer weiteren Studie von MAIER et al. (2005) wurde ein prozentualer Anteil einer Beinlängendifferenz $\geq 15 \mathrm{~mm}$ von $33 \%$ bei retrograder Marknagelung und von $20 \%$ bei antegrader Nagelung publiziert. Der Anteil in unserer Studie liegt etwas höher, anzumerken ist, dass bereits Beinlängendifferenzen von $0,5 \mathrm{~cm}$ mit in die Bewertung aufgenommen wurden.

Die Bewegungsmessung nach der Neutral-Nullmethode ergab bei Gruppe 1 bei $10 \%$ der Patienten auf der betroffenen Seite ein Streckdefizit des Kniegelenkes $\geq 5^{\circ}$ und in Gruppe 2 bei 5,9\%. Im Vergleich hierzu gab MAIER et al. (2005) in ihrer Studie von 2005 ein Streckdefizit $>10^{\circ}$ bei retrograder Nagelung von $10 \%$ und bei anterograder Nagelung von $5 \%$ an. Ein Beugedefizit im Kniegelenk $\geq 10^{\circ}$ fand sich in Gruppe 1 bei 28,6\% der Patienten und in Gruppe 2 bei 11,8\%, im Vergleich hierzu geben MAIER et al. (2005) für die retrograde Nagelung ein Beugedefizit $>20^{\circ}$ bei $19 \%$ der nachuntersuchten Patienten an. Hinsichtlich der Beweglichkeit im Hüftgelenk zeigte sich bei 9,5\% der Patienten der Gruppe 1 und bei 23,5\% der Gruppe 2 ein Streckdefizit $\geq 5^{\circ}$, sowie bei 76,2\% der Gruppe 1 und bei 82,3\% der Gruppe 2 eine Einschränkung der Beugung $\geq 10^{\circ}$. Eine Einschränkung der Rotation $\geq 10^{\circ}$ wiesen 42,9\% der Gruppe 1 und 52,9\% der Gruppe 2 auf. Für die Ab- und Adduktion im Hüftgelenk zeigte sich bei 9,5\% der Gruppe 1 und 17,6\% der Gruppe 2 eine Einschränkung $>5^{\circ}$. Es zeigte sich jedoch bei 52,4 \% der Gruppe 1 Patienten sowie bei $47,1 \%$ der Gruppe 2 eine seitengleiche Beweglichkeit der Hüfte. Im Vergleich hierzu geben MAIER et al. (2005) eine seitengleiche Beweglichkeit der Hüftgelenke bei $60 \%$ der Patienten nach Marknagelung an. Die in unserer Studie gemessene Beweglichkeit ist im Vergleich etwas schlechter, hierzu ist anzumerken, dass es sich in unserer Studie bei den Nachuntersuchungen nicht nur um die alleinige Femurfraktur handelt, sondern teilweise auch Begleitverletzungen im Beckenbereich vorlagen. Beim Vergleich der beiden Gruppen zeigt sich eine gering schlechtere Beweglichkeit in Gruppe 2 als in Gruppe 1, wobei jedoch keine Signifikanz nachweisbar war.

In Anlehnung an den Score nach NEER et al. (1967) erfolgte die funktionelle Auswertung des Outcomes vor allem im Hinblick auf klinisch erfassbare Veränderungen und subjektive Angaben der Patienten. 45,5\% der nachuntersuchten Patienten der Gruppe 1 erzielten laut dem berechneten Score ein sehr gutes Ergebnis, $27,3 \%$ ein gutes Ergebnis. In Gruppe 2 erzielten 26,3\% ein sehr gutes und 42,1\% ein 


\section{Diskussion}

gutes Ergebnis. Der Mittelwert in Gruppe 1 betrug 60,45 von 85 Punkten und in Gruppe 2 56,05 von 85 Punkten, ein signifikanter Unterschied war mit $p=0,509$ nicht nachweisbar. Im Vergleich hierzu betrug der durchschnittliche Gesamtscore nach NEER et al. (1967) in einer Studie von SCHMIDTMANN et al. (1997) 88 von 100 Punkten und war ebenfalls als gutes Ergebnis einzustufen. 


\section{Zusammenfassung}

\subsection{Einleitung:}

In einer retrospektiven Studie wurden konsekutiv registrierte Schockraumpatienten aus dem Zeitraum September 1994 bis Dezember 2000 der Universitätsmedizin Göttingen, die eine polytraumatische Verletzungsschwere mit einem ISS $\geq 16$ Punkten sowie eine Femurfraktur aufwiesen, erfasst und hinsichtlich einer sehr frühzeitigen (innerhalb der ersten 6 Stunden) sowie einer zeitlich verzögerten operativen Versorgung der Femurfraktur untersucht. Hierbei wurden sowohl klinische Parameter aus dem stationären Aufenthalt wie auch Daten einer klinischen Nachuntersuchung und Befragung der Patienten analysiert. Die Studie zeigte unter anderem auch die verschiedenen Ansätze zur Versorgung der Femurfraktur beim Polytrauma nach ETCund DC-Konzept.

\subsection{Methodik:}

In der hier vorliegenden retrospektiven Studie wurden 91 Polytraumapatienten mit Femurfraktur, die im o.g. Zeitraum über den Schockraum der Universitätsmedizin Göttingen aufgenommen wurden, analysiert. 52 Patienten konnten nach Zeitpunkt der primären operativen Versorgung der Femurfraktur in die Gruppe 1 eingeteilt werden und 39 Patienten, die nach Ablauf der ersten 6 Stunden nach Aufnahme im Schockraum primär operativ im Bereich der Femurfraktur versorgt wurden, in Gruppe 2 eingestuft werden. Diese beiden Gruppen wurden nun anhand der aus den Akten ermittelten klinischen Parameter, einem von der Untersucherin selbst konzipierten Fragebogen sowie der standardisierten klinischen Nachtuntersuchung verglichen und statistisch mit Hilfe des Statistik-Programms Statistica 6.0 ausgewertet. Als statistische Tests zum Vergleich der beiden Gruppen wurden der Wilcoxon-MannWhitney-Test und der Chi-Quadrat-Test genutzt, das Signifikanzniveau lag bei p=0,05 Außerdem wurden der Mittelwert, Minimum, Maximum sowie die Standardabweichung berechnet.

\subsection{Ergebnisse:}

Hinsichtlich der Jahresverteilung fand sich mit $p=0,032$ eine erhöhte Patientenanzahl der Gruppe 1 im Jahre 1995 sowie der Gruppe 2 im Jahre 1998. Mit $p=0,002$ erfolgte 
bei 90,38\% der Patienten der Gruppe 1 die Primäraufnahme nach dem Trauma in der Universitätsmedizin Göttingen, während es in Gruppe 2 nur 62,16\% waren. Bei der Auswertung der Unfallursachen zeigte sich mit $p=0,018$ ein signifikanter Unterschied zwischen den beiden zu untersuchenden Gruppen, in Gruppe 1 war der Anteil der Motorradunfälle deutlich höher als in Gruppe 2. Entsprechend dem Damage ControlKonzept wurden in Gruppe $137,93 \%$ und in Gruppe 2 9,3\% primär mit einem Fixateur extern versorgt. Es zeigten sich hierbei, wie auch in der Studie von MOMMSEN et al. (2009), signifikante Unterschiede für ISS, Intensivaufenthaltsdauer und Beatmungsdauer bei Vergleich zwischen den Patienten, die nach DCO und denjenigen, welche nach ETC versorgt wurden. Die primär mit Fixateur extern versorgten Patienten zeigten einen höheren ISS, eine verlängerte Intensivaufenthaltsdauer sowie verlängerte Beatmungsdauer.

40,38\% der Patienten der Gruppe 1 erlitten im stationären Verlauf Komplikationen, hier fanden sich vor allem pulmonale Komplikationen, im Vergleich traten in Gruppe 2 in $38,46 \%$ der Fälle Komplikationen auf, hier fanden sich vor allem Infektionen sowie Multiorganversagen $(p=0,038)$.

In der Befragung der Patienten zeigte sich ein signifikanter Unterschied hinsichtlich der Möglichkeiten, die bisherigen sportlichen Aktivitäten auch nach dem Trauma fortzusetzen. In Gruppe 1 konnten dies noch 37,9\% der Patienten und in Gruppe 2 nur $13 \%(p=0,041)$.

Mit $p=0,01$ zeigte sich ein höherer Bodymassindex in Gruppe 2 mit 27,16 $\pm 3,82$ als in Gruppe 1 mit 23,67 $\pm 3,22$.

Bei der Analyse weiterer klinischer Parameter wie auch des Outcomes zeigten sich zwischen den beiden Gruppen keine weiteren signifikanten Unterschiede.

\subsection{Schlussfolgerung:}

Das Outcome in Anlehnung an den Neer-Score (NEER et al. 1967) war mit 60,45 Punkten in Gruppe 1 etwas besser als mit 56,05 Punkten in Gruppe 2, eine Signifikanz konnte jedoch nicht nachgewiesen werden. Folglich konnte kein direkter Zusammenhang zwischen den Spätergebnissen und der frühzeitigen bzw. verzögerten Primärversorgung der Femurfraktur nachgewiesen werden. Auch im Vergleich mit den 


\section{Zusammenfassung}

Studien von MAIER et al. (2005) und SCHMIDTMANN et al. (1997) zeigt sich ein etwas schlechteres Abschneiden der im Rahmen dieser Arbeit untersuchten Gruppen. Hierbei ist die Ursache sicherlich darin zu sehen, dass bei Polytraumapatienten auch die zusätzlichen Verletzungen einen Einfluss auf die zeitliche Versorgung der Fraktur nach ausreichender vitaler Stabilisierung der Patienten im Schockraum und auf der Intensivstation auf die Spätergebnisse haben. Dementsprechend kann es kein rigides Konzept für den Zeitrahmen der Operation und auch für die Wahl der angewandten Osteosynthese-Verfahren geben, sondern es muss jeweils im Einzelfall unter der Berücksichtigung der Begleitverletzungen und der vitalen Indikation entschieden werden. 
Anhang

\section{Anhang}

6.1. Glasgow Coma Scale (TEASDALE und JENNETT 1974)

\begin{tabular}{|l|c|l|}
\hline AUGEN & 4 & spontan offen \\
& 3 & auf Anruf offen \\
& 2 & auf Schmerz offen \\
\hline BEWUSSTSEIN & 1 & Geschlossen \\
\hline & 4 & Orientiert \\
& 3 & Desorientiert \\
& 2 & Unartsalat \\
& 1 & nicht ansprechbar \\
\hline MOTORIK & 6 & führt Befehle aus \\
& 5 & gezielte Schmerzabwehr \\
& 4 & ungezielte Schmerzabwehr \\
3 & beugt auf Schmerz \\
2 & streckt auf Schmerz \\
\hline
\end{tabular}


6.2. Anforderungen an Krankenhäuser zur Behandlung von Schwerverletzten im Rahmen des Traumanetzwerkes (aus "Weißbuch" Schwerverletzten-Versorgung (Deutsche Gesellschaft für Unfallchirurgie e.V. Sept.2006, S. 32)).

\begin{tabular}{|c|c|c|c|}
\hline Ausstattung & $\begin{array}{l}\text { Überregionales } \\
\text { Traumazentrum }\end{array}$ & $\begin{array}{l}\text { Regionales } \\
\text { Traumazentrum }\end{array}$ & $\begin{array}{l}\text { KH der } \\
\text { Basisversorgung }\end{array}$ \\
\hline \multicolumn{4}{|l|}{ Notfallaufnahme } \\
\hline Blutbank & $\mathbf{U}$ & $\mathbf{U}$ & Depot \\
\hline Labor & $\mathbf{U}$ & $\mathbf{U}$ & U \\
\hline Mikrobiologie & $\mathbf{U}$ & $\mathbf{U}$ & $\mathbf{W}$ \\
\hline \multicolumn{4}{|l|}{ RTH-Landeplatz } \\
\hline 24-h-Betrieb & $\mathbf{U}$ & $\mathbf{W}$ & $\mathbf{W}$ \\
\hline Tagesbetrieb & $\mathbf{U}$ & $\mathbf{U}$ & $\mathbf{W}$ \\
\hline $\begin{array}{l}\text { Atemwegssicherung/ } \\
\text { Beatmung }\end{array}$ & $\mathbf{U}$ & $\mathbf{U}$ & $\mathbf{U}$ \\
\hline $\begin{array}{l}\text { Pulsoxymetrie/ } \\
\text { Absauganlage }\end{array}$ & $\mathbf{U}$ & $\mathbf{U}$ & $\mathbf{U}$ \\
\hline EKG-Monitor & $\mathbf{U}$ & $\mathbf{U}$ & $\mathbf{U}$ \\
\hline Defibrillator & $\mathbf{U}$ & $\mathbf{U}$ & $\mathbf{U}$ \\
\hline $\begin{array}{l}\text { Invasive } \\
\text { Druckmessung }\end{array}$ & $\mathbf{U}$ & $\mathbf{U}$ & $\mathbf{U}$ \\
\hline \multicolumn{4}{|l|}{ Not-OP-Sets } \\
\hline Laparotomie & $\mathbf{U}$ & $\mathbf{U}$ & $\mathbf{U}$ \\
\hline $\begin{array}{l}\text { Ext.Stabil. } \\
\text { Becken }\end{array}$ & $\mathbf{U}$ & $\mathbf{U}$ & $\mathbf{U}$ \\
\hline Kraniotomie & $\mathbf{U}$ & $\mathbf{U}$ & $\mathbf{W}$ \\
\hline Tracheotomie & $\mathbf{U}$ & $\mathbf{U}$ & $\mathbf{U}$ \\
\hline Thorakotomie & $\mathbf{U}$ & $\mathbf{U}$ & $\mathbf{U}$ \\
\hline Bülaudrainage & $\mathbf{U}$ & $\mathbf{U}$ & $\mathbf{U}$ \\
\hline Endoskopie & $\mathbf{U}$ & $\mathbf{U}$ & $\mathbf{U}$ \\
\hline $\begin{array}{l}\text { Notfall- } \\
\text { medikamente }\end{array}$ & $\mathbf{U}$ & $\mathbf{U}$ & U \\
\hline \multicolumn{4}{|l|}{$\begin{array}{l}\text { Bildgebende } \\
\text { Diagnostik }\end{array}$} \\
\hline $\begin{array}{l}\text { Sono, } \\
\text { Gefäßdoppler }\end{array}$ & $\mathbf{U}$ & $\mathbf{U}$ & $\mathbf{U}$ \\
\hline Röntgen & $\mathbf{U}$ & $\mathbf{U}$ & $\mathbf{U}$ \\
\hline CT & $\mathbf{U}$ & $\mathbf{U}$ & $\mathbf{U}$ \\
\hline Angiographie & $\mathbf{U}$ & $\mathbf{U}$ & $\mathbf{W}$ \\
\hline Bildverstärker & $\mathbf{U}$ & $\mathbf{U}$ & $\mathbf{U}$ \\
\hline MRT & $\mathbf{U}$ & $\mathbf{U}$ & $\mathbf{W}$ \\
\hline $\begin{array}{l}\text { Schienen/ } \\
\text { Extensionssys. }\end{array}$ & $\mathbf{U}$ & $\mathbf{U}$ & $\mathbf{U}$ \\
\hline \multicolumn{4}{|l|}{ Temperiersysteme } \\
\hline Für Patienten & $\mathbf{U}$ & $\mathbf{U}$ & $\mathbf{U}$ \\
\hline $\begin{array}{l}\text { Für Infusionen } \\
\text { und Blut } \\
\end{array}$ & $\mathbf{U}$ & $\mathbf{U}$ & $\mathbf{U}$ \\
\hline \multicolumn{4}{|l|}{ OP-Bereich } \\
\hline \multicolumn{4}{|l|}{$\begin{array}{l}\text { OP-Ausstattung } \\
\text { Vergrößerungshilfen }\end{array}$} \\
\hline OP-Mikroskop & $\mathbf{U}$ & $\mathbf{U}$ & $\mathbf{W}$ \\
\hline Digitalisierte & $\mathbf{U}$ & $\mathbf{W}$ & $\mathbf{W}$ \\
\hline
\end{tabular}


Anhang

\begin{tabular}{|c|c|c|c|}
\hline $\begin{array}{l}\text { D2 od.D3- } \\
\text { Bildvergr. }\end{array}$ & & & \\
\hline \multicolumn{4}{|l|}{ Temperiersysteme } \\
\hline Für Patienten & $\mathbf{U}$ & $\mathbf{U}$ & $\mathbf{U}$ \\
\hline $\begin{array}{l}\text { Für Infusionen } \\
\text { und Blut }\end{array}$ & $\mathbf{U}$ & $\mathbf{U}$ & $\mathbf{U}$ \\
\hline Cell-Saver & $\mathbf{U}$ & $\mathbf{U}$ & $\mathbf{U}$ \\
\hline Rö-Bildverstärker & $\mathbf{U}$ & $\mathbf{U}$ & $\mathbf{U}$ \\
\hline $\begin{array}{l}\text { Unfallchirurg. } \\
\text { Instrumentarium }\end{array}$ & U & $\mathbf{U}$ & $\mathbf{U}$ \\
\hline Fixateur extern & $\mathbf{U}$ & $\mathbf{U}$ & $\mathbf{U}$ \\
\hline Systeme inkl.Becken & $\mathbf{U}$ & U & $\mathbf{U}$ \\
\hline $\begin{array}{l}\text { Spezielle } \\
\text { unfallchir.Implantate } \\
\text { inkl.Wirbelsäule }\end{array}$ & $\mathbf{U}$ & U & - \\
\hline \multicolumn{4}{|l|}{ Rehabilitation } \\
\hline $\begin{array}{l}\text { Physiotherapie und } \\
\text { Frührehabilitation }\end{array}$ & $\mathbf{U}$ & $\mathbf{U}$ & $\mathbf{U}$ \\
\hline Sozialdienst - AHB & $\mathbf{U}$ & $\mathbf{U}$ & $\mathbf{U}$ \\
\hline
\end{tabular}

(W: Wünschenswert U:Unbedingt erforderlich) 


\subsection{Mangled Extremity Severity Score(MESS)}

(JOHANSEN et al. 1990, S.570)

\begin{tabular}{|c|c|c|c|}
\hline \multicolumn{3}{|c|}{ Parameter } & Punkte \\
\hline \multirow[t]{4}{*}{ A } & \multirow[t]{4}{*}{$\begin{array}{l}\text { Knochen- und } \\
\text { Weichteilverletzung }\end{array}$} & $\begin{array}{l}\text { Niedrige Energie (einfache Fraktur), } \\
\text { Schusswunde }\end{array}$ & 1 \\
\hline & & $\begin{array}{l}\text { Offene oder multiple Frakturen mit starker } \\
\text { Dislokation }\end{array}$ & 2 \\
\hline & & $\begin{array}{l}\text { Hohe/mittlere Energie (Nahschuss mit Schrot, } \\
\text { Quetschtrauma) }\end{array}$ & 3 \\
\hline & & $\begin{array}{l}\text { Hochrasanztrauma (zusätzl.schwere } \\
\text { Kontamination, Weichteilavulsion }\end{array}$ & 4 \\
\hline \multirow[t]{3}{*}{ B } & \multirow[t]{3}{*}{$\begin{array}{l}\text { Extremitäten- } \\
\text { ischämie }\end{array}$} & $\begin{array}{l}\text { Pulse abgeschwächt od.fehlend, ausreichende } \\
\text { Perfusion }\end{array}$ & $1^{*}$ \\
\hline & & Pulslos, Parästhesien, verminderte Kapillarfüllung & $2^{*}$ \\
\hline & & $\begin{array}{l}\text { Kühl, motorische Lähmung, asensibel } \\
\text { ("doppeltes Scoring für Ischämie }>6 \mathrm{~h} \text { ) }\end{array}$ & $3^{*}$ \\
\hline \multirow[t]{3}{*}{$\mathbf{C}$} & \multirow[t]{3}{*}{ Schock } & Systol.RR immer $>90 \mathrm{mmHg}$ & 0 \\
\hline & & Vorübergehende Hypotension & 1 \\
\hline & & Anhaltende Hypotension & 2 \\
\hline \multirow[t]{3}{*}{$\mathbf{D}$} & \multirow[t]{3}{*}{ Alter } & $<30$ Jahre & 0 \\
\hline & & 30-50 Jahre & 1 \\
\hline & & $>50$ Jahre & 2 \\
\hline \multicolumn{2}{|c|}{ MESS-Punktzahl $\geq 7$ zeigte } & in Studien einen positiven Voraussagewert von & $100 \%$ \\
\hline
\end{tabular}




\section{4. modifizierter Score nach NEER et al. (1967)}

1. ) Schmerzen: kein Schmerz

20 Punkte

periodische auftretend bzw. wetterabhängig

16 Punkte

mit Ermüdung bzw. nach längerer Belastung

12 Punkte

eingeschränkte Funktion durch Schmerz

8 Punkte

ständig auftreten tagsüber

4 Punkte

ständig tagsüber und nachts

0 Punkte

2. ) Anatomie : Wiederherstellung

$5 \%$ Bewegungshinderung oder $0,5 \mathrm{~cm}$ kürzer

15 Punkte

$10 \%$ Bewegungshinderung oder $2 \mathrm{~cm}$ verkürzt

12 Punkte

$15 \%$ Bewegungshinderung oder $3 \mathrm{~cm}$ kürzer

9 Punkte

Vereinigung mit grober Deformität

6 Punkte

Nichtvereinigung $=$ Pseudarthrose

3 Punkte

0 Punkte

3. ) Funktion : wie vor der Verletzung

geringe Einschränkung

20 Punkte

mittlere Einschränkung, z.B. Treppe steigen,etc.

16 Punkte

schwere Einschränkung, z.B. Gehstock

12 Punkte

Nutzung 2 Unterarmgehstützen, Stützapparate,etc.

8 Punkte

Rollstuhl

0 Punkte

4. ) Arbeit :

wie vor der Verletzung

regelmäßig, aber mit Handycap

10 Punkte

andere Arbeit

leichte Arbeit bzw. verkürzte Arbeitszeit

keine Arbeit mehr möglich

8 Punkte

6 Punkte

4 Punkte

0 Punkte

5. ) Bewegung : normal oder Gesamtbeweglichkeit $360^{\circ}$

20 Punkte

$260^{\circ}-310^{\circ}$

16 Punkte

$200^{\circ}-250^{\circ}$

12 Punkte

$140^{\circ}-190^{\circ}$

8 Punkte

$80^{\circ}-130^{\circ}$

4 Punkte

$<80^{\circ}$

0 Punkte

nach Addition der Punkte :

$68-85$ Punkte $=$ sehr gutes Ergebnis

$47-67$ Punkte $=$ gutes Ergebnis

$22-46$ Punkte $=$ mäßiges Ergebnis

$0-21$ Punkte $=$ nicht zufriedenstellendes Ergebnis 


\subsection{AO-Klassifikation der Femurfraktur}

31 A: Trochanterenregion (zwischen Linea intertrochanterica und Horizontalen am unteren Rand des Trochanter minor)

31 A1: Pertrochanter einfach (stabil)

31 A2: Pertrochanter, mehrfragmentär (instabil)

31 A3: Intertrochanter d.h. distal des Tr. major und proximal des Trochanter minor

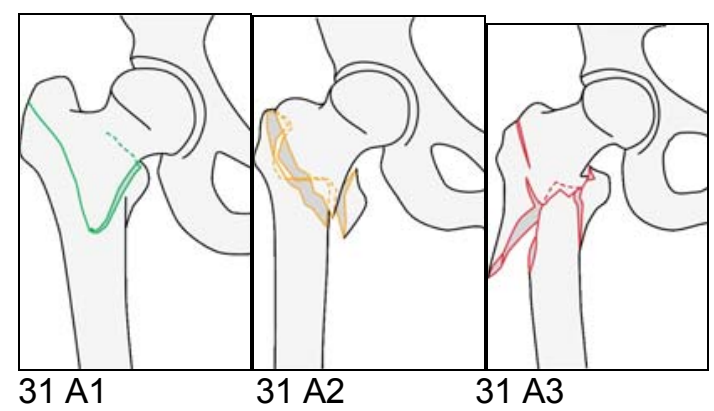

\section{B: Schenkelhals-Fraktur}

31 B1: subkapital, wenig disloziert

31 B2: transzervikal

31 B3: subkapital, disloziert

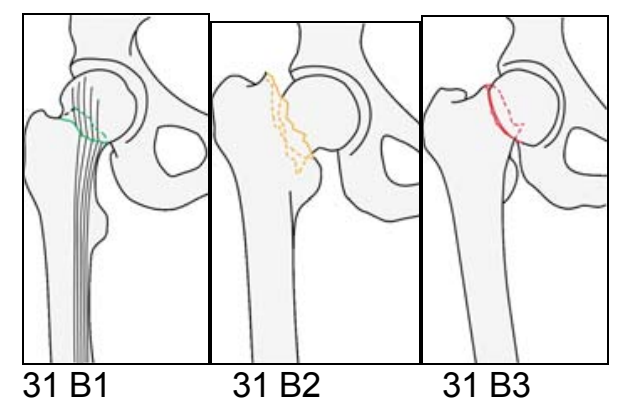

\section{C: Femurkopf-Fraktur}

31 C1: Reine Spaltung

$31 \mathrm{C2}$ : Reine Impression

31 C3: Kombination von 2 Frakturen:

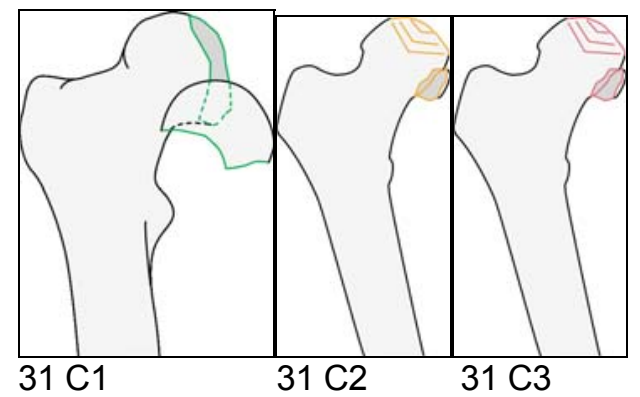


32 A einfache Femurschaftfraktur

32 A1 Spiralfraktur

32 A2 Schrägfraktur

32 A3 Querfraktur

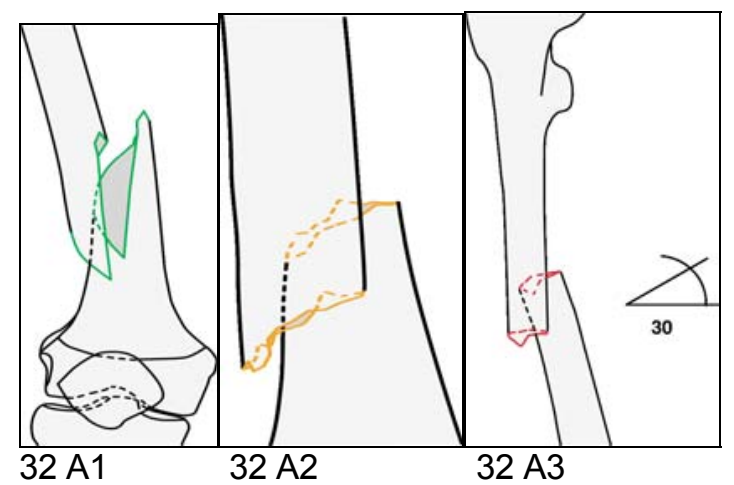

\section{B Keil-Fraktur}

32 B1 Torsionsfraktur mit Drehkeil

32 B2 mit einzelnem Biegekeil

32 B3 mit fragmentiertem Biegekeil

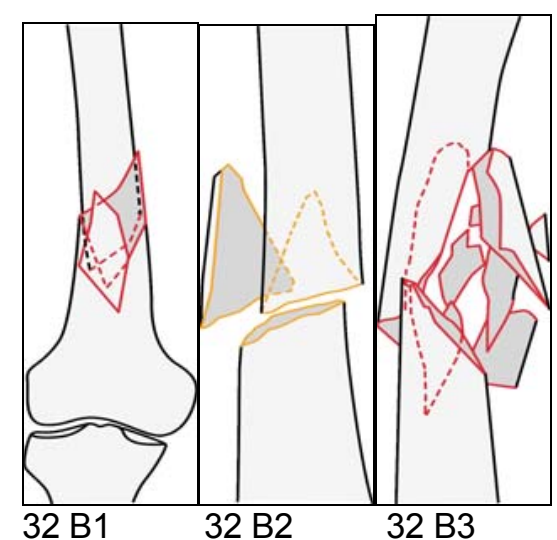

32 C Mehrfragment-Torsions-Fraktur

32 C1 Mehrfragment-Torsions-Fraktur

$32 \mathrm{C} 2$ Etagenfraktur

32 C3 irreguläre Fraktur

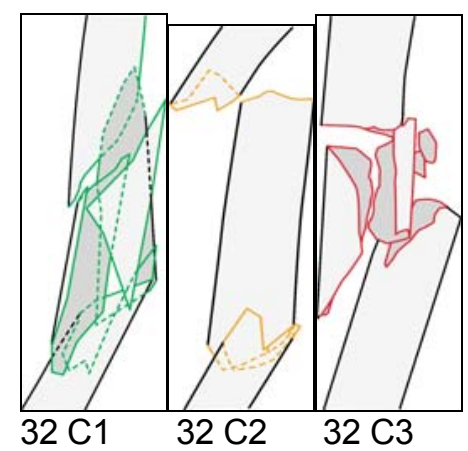


33 A1 einfache suprakondyläre Femurfraktur

33 A2 metaphysäres Keilfragment

33 A3 komplexe metaphysäre Femurfraktur

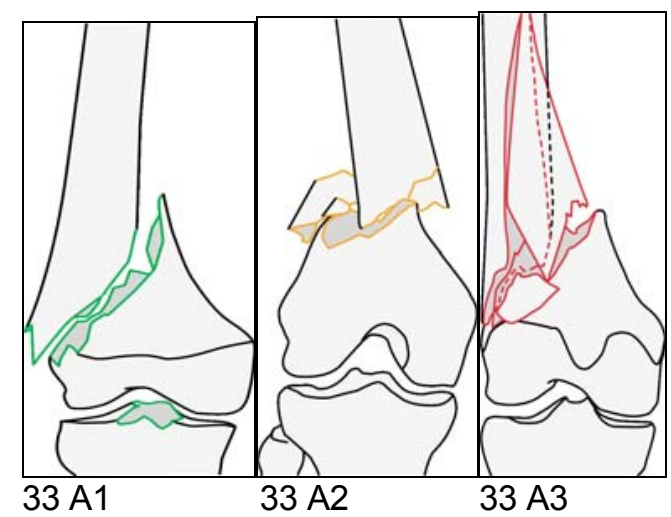

\section{B Teilgelenkbeteiligung}

33 B1 sagittal durch lateralen Kondylus

33 B2 sagittal durch medialen Kondylus

33 B3 frontaler Frakturverlauf:

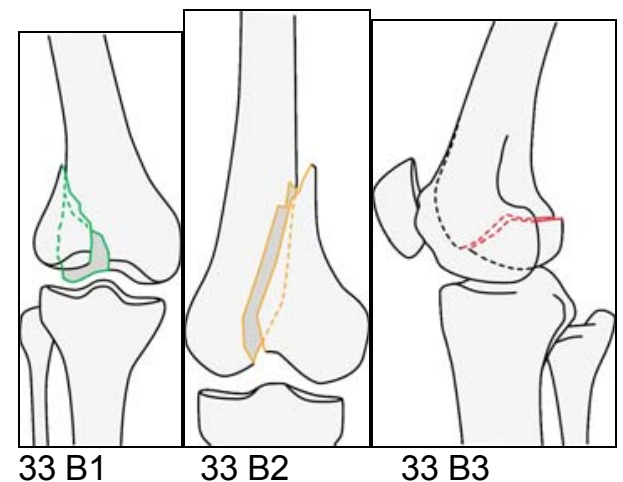

33C multifragmentäre Gelenk-Fraktur

$33 \mathrm{C} 1$ artikulär und metaphysär monofragmentär

33 C2 artikulär mono-, metaphysär multifragmentär

33 C3 multifragmentär

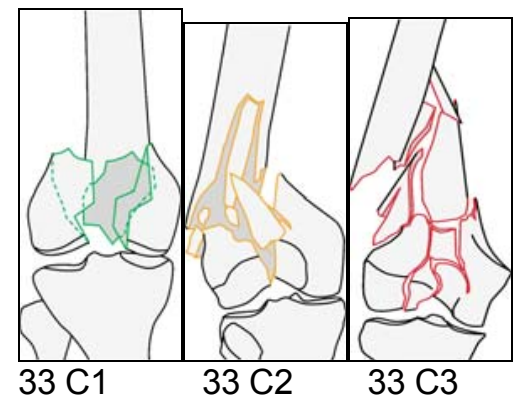

\subsection{Anschreiben an die Patienten}


Absender

Patient

Anschrift

Sehr geehrter Herr

Frau

Am xx.Xx.xxxx haben Sie einen schweren Unfall erlitten. Sie wurden damals als Polytrauma (Schwerverletzter) bei uns eingeliefert. Damals wurden Sie hier bei uns in der Universitätsklinik Göttingen notfallmäßig versorgt und behandelt.

Uns interessiert heute wie es Ihnen heute geht. Wir wollen mit den Erkenntnissen, die wir aus der Befragung vieler Verletzter erfahren, die Behandlung weiterer Unfallopfer verbessern. Optimal wäre es, wenn wir uns mit Ihnen hier in Göttingen unterhalten könnten und gleichzeitig noch einmal eine körperliche Untersuchung vornähmen. Wir wissen aus Erfahrung mit vielen Unfallverletzten, dass häufig Probleme bestehen, die im Gespräch und bei der Untersuchung geklärt werden können.

Frau Böhmert wird sich in den nächsten Tagen bei Ihnen melden und mit Ihnen einen Termin ausmachen. Wir hoffen auf Ihre Mitarbeit, denn damals konnten wir Ihnen helfen. Dieses Mal helfen Sie anderen.

Sollten Sie leider nicht zu uns nach Göttingen kommen können, würden wir sie bitten den beigefügten Fragebogen zu bearbeiten und in den frankierten Rückumschlag an uns zurückzusenden.

Mit herzlichem Dank für Ihre Mitarbeit verbleibe ich Mit freundlichen Grüßen

PD Dr.med.Dresing

\subsection{Fragebogen}


Name :

Nr.:

Vorname :

(Zutreffendes bitte jeweils ankreuzen.)

1.) Familienstand :
O Alleinstehend
O Verheiratet
O Verwitwet
O Geschieden
O Im elterlichen Haushalt lebend
O Im Pflegeheim lebend

2. ) Schulbildung :
O Hauptschulabschluss
O Realschulabschluss
O Abitur
O Kein Schulabschluss

3. ) Berufausbildung :
O keine
O Lehre :
O Abgeschlossen
O Noch nicht abgeschlossen (im .... Lehrjahr)
O Studium: O Abgeschlossen
O Noch nicht abgeschlossen (im .... Semester)

4.) Sind sie jetzt berufstätig ?

O Ja => wenn ja, welchen Beruf üben sie jetzt aus:

Welchen haben sie vor dem Unfall ausgeübt:
=> wie lange waren sie nach dem Unfall krankgeschrieben (arbeitsunfähig) ?
0 weniger als 6 Monate
o mehr als 6 Monate
o mehr als 12 Monate

O Nein => wenn nein, dann 
O Arbeitslos

O Umschulung

O Erwerbsunfähig

O Schwerbehinderung ........\%

5.) Wurden Sie nach ihrem Aufenthalt bei uns in der Universitätsklinik Göttingen noch in einem andere Krankenhaus behandelt?

$$
\begin{aligned}
& 0 \mathrm{Ja} \quad=>\text { Wie lange? } \\
& 0 \text { Nein }
\end{aligned}
$$

6.) Haben Sie nach ihrem Aufenthalt bei uns in der Universitätsklinik Göttingen eine Rehabilitationsbehandlung (KUR bzw. REHA) durchgeführt?

$$
\begin{array}{ll}
0 & \mathrm{Ja} \quad=>\text { Wie lange? } \\
0 & \text { Nein }
\end{array}
$$

7.) Erhalten Sie eine private oder berufsgenossenschaftliche Unfallrente / -entschädigung?

Private Unfallrente :
O Ja
O Nein

Berufsgenossenschaftliche Rente :
O Ja
O Nein

8.) Angaben zum Privatleben :

=> Wie hat sich ihr häusliches Umfeld nach dem Unfall geändert ?
O keine Veränderung
O Wohnungswechsel wegen Behinderung notwendig
O Betreuung \& Hilfe durch Familienangehörige notwendig
O Betreuung \& Hilfe durch Pflegedienst notwendig

=> Hat sich ihr Freizeitverhalten nach dem Unfall verändert ?

a) Hobby : vor dem Unfall :
O gleich geblieben
O nicht mehr möglich
O neues Hobby:

b) Sport :

vor dem Unfall : 
nach dem Unfall :

c) Urlaubsverhalten :
O gleich geblieben
0 eingeschränkt
O nicht mehr möglich

9.) Haben Sie in den letzte Wochen Schmerzen gehabt ?

O Nein => Wenn nein dann fahren sie mit Frage10 fort.

O $\mathrm{Ja} \quad=>$ Wie oft treten die Schmerzen auf?

O Gelegentlich

O Häufig

O Ständig

$=>$ Wie stark sind ihre Schmerzen ?
$\begin{array}{ll}1 & 2 \\ 0 & 0\end{array}$
3
$4 \quad 5$
6
Leicht
$0 \quad 0$
unerträglich

$=>$ Haben sich ihre Schmerzen im letzte Jahr verändert ?

O verringert $\quad 0$ gleich geblieben $\quad 0$ stärker geworden

=> Bitte kennzeichnen Sie auf der folgenden Abbildung die Gebiete, in denen Sie Schmerzen haben?

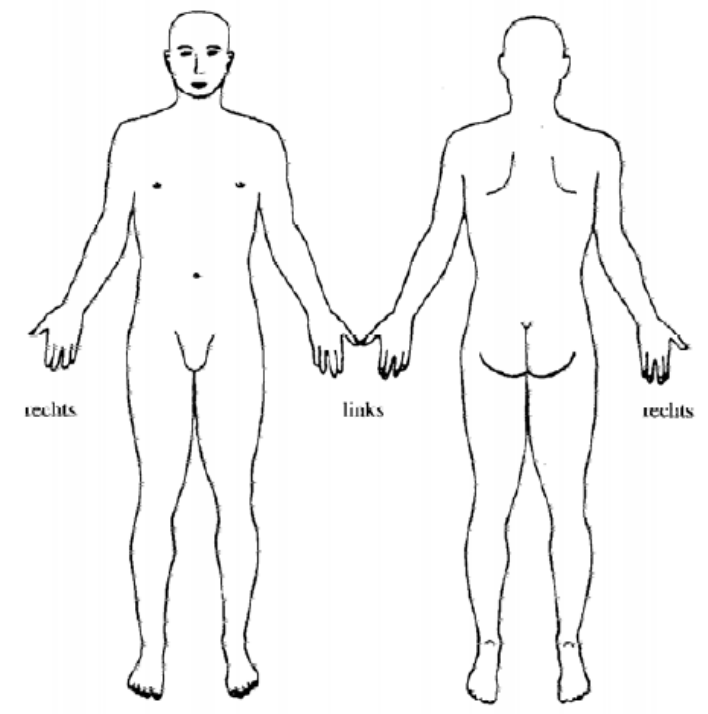

=> Wie empfinden Sie die Schmerzen ? 
Anhang

$\begin{array}{lcc}\text { dumpf, drückend } & \text { Ja } & \text { Nein } \\ \text { pochend, klopfend, stechend } & \mathbf{0} & \mathbf{0} \\ \text { krampfartig } & \mathbf{0} & \mathbf{0} \\ \text { ziehend, reißend } & \mathbf{O} & \mathbf{0} \\ \text { schrecklich, unerträglich } & \mathbf{0} & \mathbf{0} \\ & \mathbf{0} & \mathbf{0}\end{array}$

=> Welche Behandlung oder Medikamente erhalten Sie gegen die Schmerzen?

=> Falls Sie Schmerzmedikamente einnehmen, wie häufig nehmen Sie die Medikamente ein?
o täglich $1 x$
O täglich 2-3x
0 wöchentlich $1 x$
O wöchentlich 2-3x
O 1 x monatlich
O 2-3x im Monat
O mehr als 3x im Monat

=> Falls Sie Schmerzmedikamente einnehmen, wie lange dauert es bis die Schmerzen deutlich gelindert sind?
O keine Linderung
O 1 Stunde
02 Stunden
O 3-4 Stunden
O 5-12 Stunden
$\mathbf{O}>12$ Stunden

$=>$ Wie stark sind Ihre Schmerzen in Ruhe bzw. in der Nacht?
O keine
O leichte
O stärkere
O sehr starke

=> Wie stark sind Ihre Schmerzen bei Belastung bzw. Bewegung ?
O keine
O leichte
O stärkere
O sehr starke 
10.) Wie schätzen Sie das Ergebnis der Behandlung ihrer Verletzungen am Bein ein?

O Beweglichkeit/Belastbarkeit wie vor dem Unfall

O Gehstrecke reduziert auf 2000m (Gehen ohne Pause)

O Gehstrecke reduziert auf $1000 \mathrm{~m}$

O Gehstrecke reduziert auf $500 \mathrm{~m}$

O Gehstrecke reduziert auf $<500 \mathrm{~m}$

O Starke Einschränkungen (Gehstrecke <100m)

O Keine Belastbarkeit möglich (fahre im Rollstuhl)

11.) Wie schätzen Sie ihre körperliche Belastbarkeit zur Zeit ein ?

O Unverändert

O Leichte Einschränkungen

O Stärkere Beeinträchtigung (beim Treppesteigen häufigere Pausen)

O Starke Beeinträchtigung (Treppensteigen stark behindert, Stehen nur kurz möglich)

O Stärkste Beeinträchtigung (Stehen nicht mehr möglich)

12.) Haben Sie Missempfindungen ?
O nein
O Kribbeln
O Taubheitsgefühl
O Gefühllosigkeit

13.) Bitte zeichnen Sie die Stellen der Missempfindungen in die Abbildung ein.

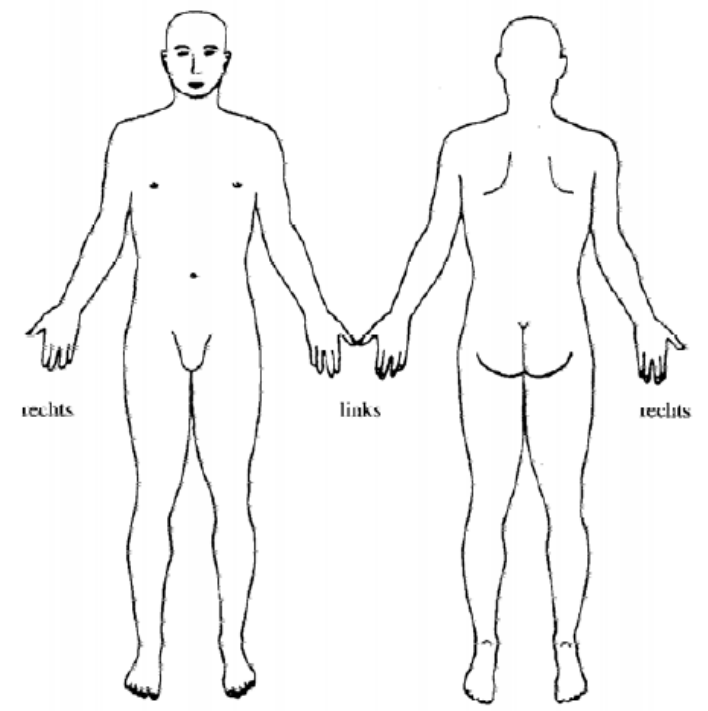


Anhang

14.) Benötigen Sie Hilfsmittel ?
O keine
O Gehstock
O Unterarmgehstützen (1oder2)
O Rollstuhl
O sonstige (z.B. Schienen, etc.)

15.) Sind Sie mit der Versorgung hier in der Universitätsklinik Göttingen nach dem Unfall zufrieden gewesen?
$\mathrm{O} \mathrm{Ja}$
O Nein, warum?

Vielen Dank für Ihre Bemühungen diesen Fragebogen zu beantworten.

\subsection{Nachuntersuchungsbogen}


Name :

Vorname :

Größe : .

.cm Gewicht : ...

..kg Geschlecht :

Alter : Jahre

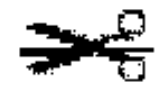

Schmerzen : A) O Ja O Nein

B) Auftreten: $\quad 0$ keine $\quad 0$ periodisch bzw. wetterabhängig auftretend

O mit Ermüdung verbunden/bei längerer Belastung

O eingeschränkte Funktion durch Schmerz

O ständig tagsüber $\quad \mathbf{O}$ ständig nachts

C) Lokalisation :
D) Art :
Bewegungssch
Anlaufschmerz
Ruheschmerz
O Ja
O Nein
O Ja O Nein
O Ja O Nein

Funktion : $\quad \mathbf{O}$ wie vor der Verletzung $\quad \mathbf{O}$ geringe Einschränkungen

O beschränkt, v.a. Treppe steigen etc.

O Nutzung eines Gehstocks, schwerere Einschränkung

O stärkste Einschränkung (Stützen, Rollstuhl, etc.)

Arbeit : $\quad \mathbf{O}$ wie vor der Verletzung $\quad \mathbf{O}$ regelmäßig aber mit Handycap

$\mathbf{O}$ andere Arbeit $\quad \mathbf{O}$ leichtere Arbeit mit verkürzter Arbeitszeit

O keine Arbeit mehr möglich, erwerbsunfähig

Inspektion : O unauffällig

O auffällig (Achsabweichung, etc.) $=>$

Hilfsmittel: 0 keine $\quad 01$ Gehstock

o 1 Unterarmgehstütze

O 2Unterarmgehstützen O Gehwagen/Rollator O Rollstuhl

O sonstiges (Schienen, Orthesen, etc.):

Gangbild : O unauffällig $\quad$ O auffällig =>

Kniebeuge : $\mathbf{O}$ möglich $\quad \mathbf{O}$ eingeschränkt $\quad \mathbf{O}$ nicht möglich

Einbeinstand : $\mathbf{O}$ möglich $\quad \mathbf{O}$ eingeschränkt $\quad \mathbf{O}$ nicht möglich

(=> Trendelenburgzeichen positiv : ....... Seite)

Hackenstand: $\mathbf{O}$ möglich $\quad \mathbf{O}$ eingeschränkt $\mathbf{O}$ nicht möglich

Zehenspitzenstand : $\quad \mathbf{O}$ möglich $\quad \mathbf{O}$ eingeschränkt $\quad \mathbf{O}$ nicht möglich

Fingerbodenabstand : . $\mathrm{cm}$

Aufrichten aus dem Sitzen : $\quad$ O möglich $\quad$ O mit Hilfe, eingeschränkt $\quad$ O nicht möglich 
Beckenstand : $\quad \mathbf{O}$ symmetrisch $\quad \mathbf{O}$ rechts höher als links

O links höher als rechts Differenz : ........cm

Wirbelsäule : $\quad$ O unauffällig $\quad$ O auffällig =>

Druckschmerz: Oberschenkel: O Ja O Nein

Leistendruckschmerz: O Ja O Nein

Kniegelenk: $\quad O \mathrm{Ja} \quad \mathrm{O}$ Nein

Neurologie : A) Sensibilität : O unauffällig O Ausfälle :

B) Reflexe :

\begin{tabular}{|l|l|l|}
\hline & rechts & links \\
\hline PSR & & \\
\hline ASR & & \\
\hline
\end{tabular}

C) Muskelfunktion : $\mathbf{O}$ volle Kraft

O Ausfälle :

Messblatt für untere Extremität (nach Neutral-Null-Methode):

Hüftgelenke:

Streckung/Beugung

Abspreizen/Anführen

Drehg.auswärts/einwärts (Hüftgelenk $90^{\circ}$ gebeugt)

Drehg. auswärts/einwärts (Hüftgelenk gestreckt)
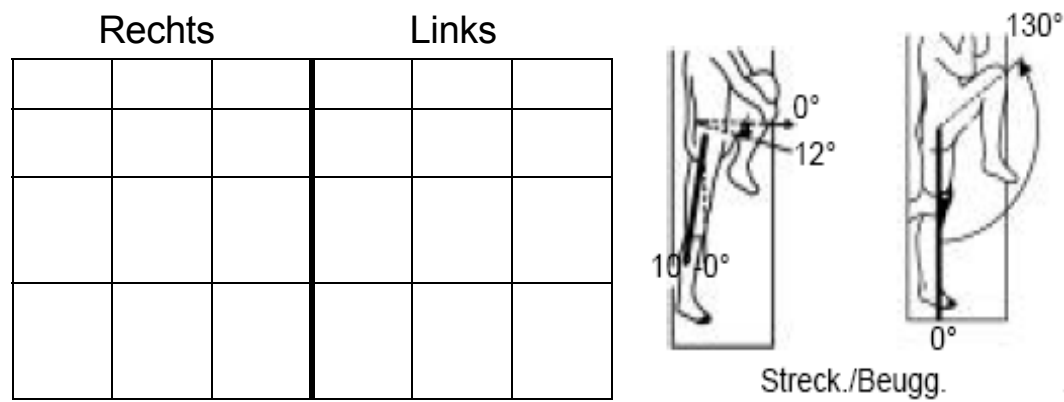

Streck./Beugg.

\section{Kniegelenke : \\ Streckung/Beugung}

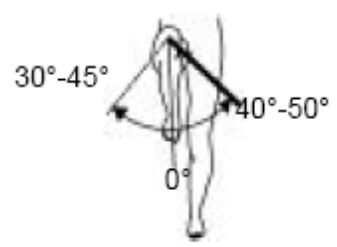

Drehg. ausw./einw.
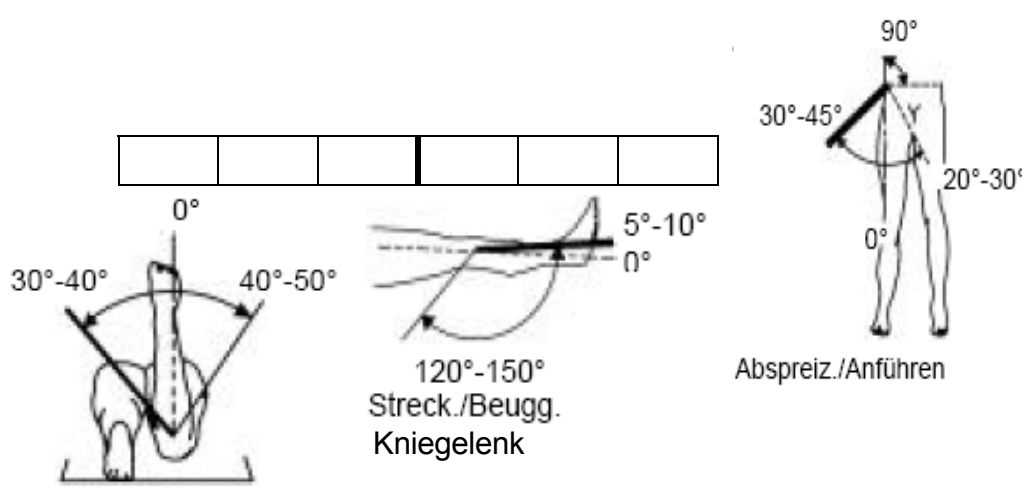

Abspreiz./Anführen

Streck./Beugg

Beinlänge in $\mathrm{cm}$ : 


\section{Anhang}

Vord.ob.D.-beinstachel bis Außenknöchelspitze

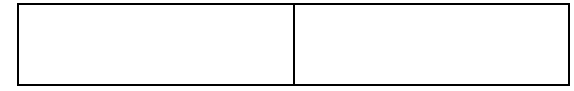

Umfangmaße in $\mathrm{cm}$ :

$20 \mathrm{~cm}$ ob.innerer Kniegelenksspalt

$10 \mathrm{~cm}$ ob.innerer Kniegelenksspalt

Kniescheibenmitte

$15 \mathrm{~cm}$ unterh.inn.Kniegelenksspalt

\begin{tabular}{|l|l|}
\hline & \\
\hline & \\
\hline & \\
\hline & \\
\hline
\end{tabular}

Gefäßstatus :

\begin{tabular}{|l|l|l|}
\hline & Rechts & Links \\
\hline A.femoralis & & \\
\hline A.poplitea & & \\
\hline A.tibialis posterior & & \\
\hline A.dorsalis pedis & & \\
\hline
\end{tabular}

Operationen am Oberschenkel :

Anzahl :

Narbenanzahl :

Gesamtlänge der Narben : . $\mathrm{cm}$

Sonstige relevante Befunde (Drehfehler, Achsabweichung, etc.):

Krankenhausaufenthalte außerhalb ? O nein

$$
\begin{array}{r}
\mathrm{O} \mathrm{Ja}= \\
\text { Wo } \text { Wann ?........... } \\
\text { Wie lange? }
\end{array}
$$

REHA / AHB ? O Nein O Ja => Dauer?

Materialentfernung am Oberschenkel ? O Nein

$\mathrm{O} \mathrm{Ja}=>$ Wann?

Anerkennung Schwerbehinderung ? O Nein

$\mathrm{O} \mathrm{Ja}$ 


\section{LITERATURVERZEICHNIS}

Baker SP, Haddon W, O'Neill B, Long WB (1974): The injury severity score : a method for describing patients with multiple injuries and evaluating emergency care. $\mathrm{J}$ Trauma 14: 187-196

Beck A, Gebhard F, Kinzl L (2002): Notärztliche Versorgung des Traumapatienten. Notfall Rettungsmed 5: 57-71

Bernhard M, Helm M, Aul A, Gries A (2004): Präklinisches Management des Polytraumas. Anaesthesist 53: 887-904

Biewener A, Aschenbrenner U, Rammelt S (2004): Impact of helicopter transport and hospital level on mortality of polytrauma patients. JTrauma 56: 94-98

Bone LB, Johnson KD, Weigelt J, Scheinberg R (1989): Early versus delate stabilization of femoral fractures: $\boldsymbol{A}$ prospective randomized study. J Bone Joint Surg Am 71: 336-340

Bouillon B, Neugebauer E (1998): Outcome after Polytrauma. Langenbeck's Arch Surg 383: 228-234

Bouillon B, Kanz KG, Lackner CK, Mutschler W, Sturm J (2004): Die Bedeutung des Advanced Trauma Life Support (ATLS) im Schockraum. Unfallchirurg 107: 844-850

Bross S: Vergleich der Wertigkeit verschiedener Polytraumascores.

Med. Diss. Dresden 1995 (DISS 95 B 476)

Bundesministerium für Arbeit : Jahresbericht 2004, 28

Burghofer K, Finkenzeller C, Ruppert M, Schlechtriemen T, Stolpe E, Lackner CK (2006): "Hidden Intervals" - verdeckte Zeitintervalle in der Luftrettung. Notarzt 22: 175-185

Champion HR, Sacco WJ, Cornazzo AJ, Copes WS, Fonty WJ (1981): Trauma score. Crit Care Medizin 9: 672-676

Champion HR, Sacco WJ, Copes WS, Gann DS, Gennarelli TA, Flanagan ME (1989): $\boldsymbol{A}$ Revision of the trauma score. J Trauma 29: 623-629

Cowley RA (1977): Trauma center : a new concept for the delivery of critical care. J Med Soc NJ 74: 979-987

Crump JM, Duncan DA, Wears R (1988): Analysis of multiple organ system failure in trauma and nontrauma patients. Am Surg 54: 702-708

Deane SA, Gaudry PL, Roberts RF, Juul O, Little JM (1986): Trauma triage - a comparison of the trauma score and vital sign score. Aust N z J Surg 56: 191-197

Deitch EA (1992): Multiple organ failure. Pathophysiology and potential future therapy. Ann Surg 216: 117-134 
Deutsche Gesellschaft für Unfallchirurgie e.V. (September 2006): "Weißbuch" Schwerverletzten-Versorgung - Empfehlungen zur Struktur, Organisation und Ausstattung der Schwerverletzten-Versorgung in der Bundesrepublik Deutschland. DGU Berlin, September 2006

Dittel KK, Weller S (1981): Zur Problematik des polytraumatisierten Patienten. Aktuelle Traumatol 11: 35

Dittmer H, Faist E, Lauterjung KL (1983): Das Mehrfach-Organversagen - die wichtigste Komplikation beim polytraumatisierten Patienten.

Zentralbl Chir 108: 385-395

Dörner K : „Klinische Chemie und Hämatologie“; 6.Aufl., Thieme Verlag, Stuttgart 2006, 312-315

Dresing K (federführender Autor), Leitlinien-Kommission der DGU: Stürmer KM, Meenen NM, Blauth M, Siebert H, Bonnaire F, Suren EG, Braun W, Wittner B, Dresing K (2007): DGU Leitlinie Polytrauma. AWMF-Leitlinien-Register Nr.012/019: 1-26; DGU Berlin 2007

Finkenzeller C, Burghofer K, Köhler M, Ruppert M, Stolpe E, Lackner CK (2005): Verzögerungen im Prähospitalzeitintervall bei Luftrettungseinsätzen. Notarzt 21: 195-205

Frank J, Marzi I, Mutschler W (1996): Schockraummanagement des Polytraumas. Zentralbl Chir 121: 943-949

Friedl HP, Trentz O (1989): Definition und Pathophysiologie des Polytraumas. OP-Journal 5 Nr. $2: 4-5$

Geldner G, Schwarz U (2003): Präklinische Polytraumaversorgung : Eilen oder Verweilen? Anästhesiol Intensivmed Notfallmed Schmerzther 38: 196-197

Giannoudis PV, Giannoudi M, Stavlas P (2009): Damage control orthopaedics : Lesson learned. Injury 40S4: 47-52

Glinz W, Affentranger T (1975): The fate of patients with severe multiple injuries, 5 years after intensiv care. Bull Soc Int Chir 34: 545-548

Grotz M, Schwermann T, Lefering R, Ruchholtz S, Graf v d Schulenburg JM, Krettek C, Pape HC (2004): DRG-Entlohnung beim Polytrauma - Ein Vergleich mit den tatsächlichen Krankenhauskosten anhand des DGU-Traumaregisters. gms-online (www.egms.de/de/meetings/dgu2004/04dgu0132.html)

Haas NP (1997): Empfehlungen zur Struktur, Organisation und Ausstattung der präklinischen und klinischen Patientenversorgung an Unfallchirurgischen Abteilungen in Krankenhäusern der Bundesrepublik Deutschland. Unfallchirurg 100(1): 2-7

Haas NP, Lindner T, Bail HJ (2007): Neues zum Polytrauma - Prioritäten in der definitiven Versorgung. Chirurg 78: 894-901

Häusler J-M C, Zimmermann H, Töbler B, Arnet B, Hüsler J (2001): Die Volkswirtschaftlichen Kosten vom Polytrauma. Print Suva Schweizerische Unfallversicherungsanstalt Luzern, Dezember 2001

Hauke J, Helm M, Lampl L (2001): Der eingeklemmte PKW-Insasse aus Sicht des Notarztes. Notarzt 17: 47-52 
Havemann D : Zur Epidemiologie des Straßenverkehrsunfalls. 1.Aufl., Thieme-Verlag, Stuttgart, New York 1972, 158-163

Hutschenreuter G : Bluttransfusion In : Chirurgie, Kapitel 2 : Operativer Eingriff Hrsg. Von Schumpelick V, Bleese N, Mommsen U ; Enke im Georg Thieme Verlag, Stuttgart New York 2000, 106-122

Johansen K, Daines M, Howey T, Helfet D, Hansen S (1990): Objective criteria accurately predict amputation following lower extremity trauma. J Trauma 30: 568-573

Johnson KD, Cadambi A, Seibert GB (1985): Incidence of adult respiratory distress syndrome in patients with multiple musculoskeletal injuries : Effect of early operative stabilisation of fractures. J Trauma 25: 375-384

Kanz KG, Sturm JA, Mutschler W, AG Notfall der DGU (2002): Algorithmen für die präklinische Versorgung von Polytrauma. Unfallchirurg 105: 1007-1014

Keel M, Trentz O (2005): Pathophysiologie of polytrauma. Rev Injury 36: 691-709

Keul W, Bernhard M, Völkl A, Gust R, Gries A (2004): Methoden des Atemwegsmanagements in der präklinischen Notfallmedizin.

Anaesthesist DOI 10.1007/s00101-004-0734-9

Kreimeier U, Lackner CK, Prückner S (2003): Neue Strategien in der Volumentherapie beim Polytrauma. Notfall Rettungsmed $\underline{6: 77-88}$

Kühne CA, Ruchholtz S, Buschmann C, Sturm J, Lackner CK, Wentzensen A, Bouillon B, Weber M (2006): Polytraumaversorgung in Deutschland - Eine Standortbestimmung. Unfallchirurg 109: 357-366

Lehnert M, Marzi I : Kapitel 21: Polytrauma In : Orthopädie und Unfallchirurgie; Hrsg. Scharf P, Rüter A; Elsevier Urban\&Fischer Verlag, München-Jena 2009, 386-414

Luiz Th (2003): Notfallmedizin morgen. Anaesthesiol Intensivmed Notfallmed

Schmerzther 38: 296-302

Maier DG, Reisig R, Keppler P, Gebhard F (2005): Posttraumatische Achsabweichungen und funktionelle Untersuchungen nach ante- bzw. retrograder Marknagelung des distalen Femurdrittels Unfallchirurg 108: 109-117

McNamara MG, Heckman JD, Corley FG (1994): Severe open fractures of the lower extremity : a retrospektive evaluation of the Mangled Extremity Severity Score (MESS).

J Orthop Trauma 8: 81-87

Milholland AV, Cowley RA, Sacco WJ (1979): Development and prospective study of anatomical index and an acute trauma index. Am Surg 45: 246

Mönk S (2003): Die Bedeutung von Simulatoren. Notfall Rettungsmed 6: 37-39

Mommsen P, Andruszkow H, Frink M, Probst C, Krettek C, Hildebrand F: Damage Control (DCO) versus Early Total Care (ETC) beim Polytrauma mit Femurschaftfraktur retrospektive Outcome-Analyse. In : Deutscher Kongress für Orthopädie und Unfallchirurgie. (73.Jahrestagung der Deutschen Gesellschaft für Unfallchirurgie, 95.Tagung der Deutschen Gesellschaft für Orthopädie und Orthopädische Chirurgie, 50.Tagung des Berufsverbandes der Fachärzte für Orthopädie.) Berlin 21.-24.10.2009. DocWI19-1016 
Moore EE, Burch JM, Franciose RJ, Offner PJ, Biffl WL (1998): Staged physiologic restoration and damage control surgery. J Surg 22: 1184-1191

Müller-Berghaus G, Lasch HG : Blutgerinnungs- und Mikrozirkulationsstörungen beim polytraumatisierten Patienten. In : „Mehrfachverletzungen. Anästhesiologie und Intensivmedizin“ Bd. 127; Hrsg. Streicher HJ, Rolle J; Springer-Verlag, Berlin, Heidelberg, New York 1980, 66-76

Muhr G : Kapitel 7: Die Bedeutung von Biologie und Biomechanik in der Frakturheilung. In : „Unfallchirurgie in Deutschland Bilanz und Perspektiven“ Hrsg. Oestern HJ, Probst J; Springer-Verlag, Berlin, Heidelberg, New York 1997, 385-389

Mutzbauer TS, Helm M (2001): Präklinisches Airway-Management bei Patienten mit Einklemmungstrauma - Darstellung aus Sicht des Anästhesisten als Notarzt an einer Rettungshubschrauberstation. Notarzt 17: 57-62

Nast-Kolb D, Ruchholtz S, Waydhas C (2005): Damage control orthopaedics. Unfallchirurg 108: 804-811

Neer CS, Grantham SA, Shelton ML (1967): Supracondylar fracture of the adult femur : A study of one hundred an ten cases. JBone Jt Surg. 49-A:591-613

Nehrlich M, Maghsudi M (1997): Polytraumamanagement - Präklinisches Handling und Schockraumversorgung. Notfall Rettungsmed 10: 45-554

Neuhof H (1991): Actions and interactions of mediator systems and mediators in the pathogenesis of ards and multiorgan failure. Acta Anaesthesiol Scand Suppl 95: 7-13

O'Brien PJ (2003): Fracture fixation in patients having multiple injuries.

Can J Surg 46: 124-128

Oestern HJ : Das Polytrauma - Präklinisches und Klinisches Management. 1.Aufl., Deutsche Gesellschaft für Unfallchirurgie; Urban\&Fischer Verlag, München 2008, 346-371

Oestern HJ, Tscherne H, Sturm J (1984): Mehrfachverletzungen. Dringlichkeitsstufen der chirurgischen Versorgung. Chir Prax 33: 127-146

Pape HC, Krettek C (2003): Frakturversorgung des Schwerstverletzten-Einfluss des Prinzips der "verletzungsadaptierten Behandlungsstrategien“ („damage control orthopaedic surgery"). Unfallchirurg 106: 87-96

Pape HC, Aufmolk M, Paffrath T, Regel G, Sturm JA, Tscherne H (1993): Primary intramedullary femur fixation in multiple trauma patients with associated lung contusion- a cause of posttraumatic ARDS? J Trauma 34: 540-548

Pape HC, Stalp M, Dahlweid M (1999): AG Polytrauma der DGU : Welche primäre Operationsdauer ist hinsichtlich eines "Borderline Zustandes" polytraumatisierter Patienten vertretbar? Eine prospektive Evaluation anhand des Traumaregisters der DGU. Unfallchirurg 102: 861-869

Pape HC, Giannoudis P, Krettek C (2002): The Timing of fracture treatment in polytrauma patients: relevance of damage control orthopaedics surgery. Am J Surg 183: 622-629 
Pape HC, Grotz M, Schwermann T (2003): Entwicklung eines Modells zur Berechnung der Kosten der Versorgung schwer Verletzter - eine Initiative des Traumaregisters der DGU. Unfallchirurg 106: 348-357

Pape HC, Hildebrand F, Krettek C (2004): Entscheidungsfindung und Prioritäten der operative Behandlungsstrategien während und nach der Schockraumversorgung. Unfallchirurg 107: 927-936

Parr MJA, Alabdi T (2004): Damage control surgery and intensiv care. Injury 35: 713-722

Piek J, Jantzen JP (2000): Empfehlungen zur Erstversorgung des Patienten mit SchädelHirntrauma bei Mehrfachverletzung. Notfall Rettungsmed 3: 32-37.

Pirente N, Gregor A, Bouillon B, Neugebauer E (2001): Lebensqualität schwerstverletzter Patienten ein Jahr nach Trauma Unfallchirurg 104: 57-63

Raschke MJ, Haas NP : Unfallheilkunde Kap.40.2.6 Komplikationen bei Frakturen In Chirurgie 7.Aufl., Hrsg. Siewert JR; Springer Verlag, Berlin 1997, 816-817

Rehn J : Unfallarten und Unfallgeschehen In : „Chirurgie der Gegenwart“ Bd.4 Hrsg. Zenker R, Deucher F, Schink W; Urban\&Schwarzenberg-Verlag, München, Berlin,

Wien 1973, 430-435

Riede UN, Schaefer HE : Allgemeine und spezielle Pathologie. 4.Aufl., Georg Thieme Verlag, Stuttgart, New York 1999, 415-416

Rixen D, Grass G, Sauerland S (2005 a): Evaluation of criteria for temporary external fixation in risk adapted damage control orthopaedic surgery of femoral shaft fractures in multiple trauma patients: "Evidence based medicine" vs. "reality" in the trauma registry of the German Trauma Society. J Trauma (in press)

Rixen D, Sauerland S, Oestern H-J, Bouillon B (2005 b): Versorgungsstrategien in der ersten operativen Phase nach Verletzung langer Röhrenknochen der unteren Extremität beim Polytrauma, Unfallchirurg 108: 829-842

Ruchholtz S (2004): Polytrauma unter dem Diktat der DRG. Ökonomische Unwägbarkeiten aus ärztlicher Sicht. 7.Deutscher Interdisziplinärer Kongress für Intensivund Notfallmedizin 01.-04.12.2004 Hamburg.

Ruchholtz S, Nast-Kolb D, Waydhas C, Stuber R, Lewan U, Schweiberer L (1995): Cost analysis of clinical treatment of polytrauma patients. Chirurg 1995, 66: 684-692

Ruchholtz S, Nast-Kolb D, Waydhas C, Schweiberer L (1996): Das Verletzungsmuster beim Polytrauma : Stellenwert der Information über den Unfallhergang bei der klinischen Akutversorgung. Unfallchirurg 99: 633-641

Rüedi TP, Murphy WM, Colton CL, Fernandez Dell'Oca A, Holz U, Kellam JF, Ochsner PE: AO Prinzipien des Frakturmanagements. Kap.4.6.1.-4.6.3. Georg-Thieme-Verlag, StuttgartNew York 2003, 445-484

Schlag G, Redl H (1988): Current findings in the pathogenesis of the shock process in traumatology. Unfallchirurgie 14: 3-11

Schlechtriemen T, Schaefer S, Stolpe E, Altemeyer KH (2002): Präklinische Versorgung von Traumapatienten in der Luftrettung. Unfallchirurg 105: 974-985 
Schmelz A, Ziegler D, Beck A, Kienzl L, Gebhard F (2002): Akutstationäre Behandlungskosten polytraumatisierter Patienten. Unfallchirurg 105: 1043-1048

Schmidtmann U, Knopp W, Wolff C, Stürmer KM (1997): Ergebnisse der elastischen Plattenosteosynthese einfacher Femurfrakturen beim Polytrauma - Ein alternatives Verfahren. Unfallchirurg 100: 949-956

Schweiberer L, Nast-Kolb D, Duswald K-H, Waydhus Ch, Müller K (1987): Das Polytrauma Behandlung nach dem diagnostischen und therapeutischen Stufenplan.

Unfallchirurg 90: 529-538

Seebach C, Marzi I : Kapitel 4 Pathophysiologie des Knochens In : Orthopädie und Unfallchirurgie; Hrsg. Scharf P, Rüter A; Elsevier Urban\&Fischer Verlag, München-Jena 2009, 40-52

Seefelder C, Matzek N, Rossi R (1988): Polytrauma - Bewertungsskalen.

Notfallmedizin 14: 227-236 und 317-328

Simmel S, Bühren V (2009): Polytrauma überlebt - und was kommt dann? Die Rehabilitation Schwerstverletzter. Unfallchirurg 112: 965-974

Stalp M, Koch C, Regel G, Krettek C, Pape HC und AG Polytrauma der DGU (2001): Entwicklung eines standardisierten Instruments zur quantitativen und reproduzierbaren Rehabilitationserfassung nach Polytrauma (HASPOC). Chirurg 72: 312-318

Statistisches Bundesamt Deutschland im Internet: http://www.destatis.de (Abrufdatum 10.08.2009)

Stürmer KM, Dresing K, Blauth M, Bonnaire F, Braun W, Meenen NM, Siebert H, Suren EG, Wittner B (2001): Polytrauma - Leitlinien für die Unfallchirurgische Diagnostik und Therapie. Unfallchirurg 104: 902-912

Sturm JA, Lackner CK, Bouillon B, Seekamp A, Mutschler WE (2002): Advanced Trauma Life Support (ATLS). Unfallchirurg 105: 1027-1032

Taeger G, Ruchholtz S, Waydhas C (2005): Damage control orthopaedics in patients with multiple injuries is effective, time saving, and safe. J Trauma 59: 409-416

Teasdale G, Jennett B (1974): Assessment of coma and impaired consciousness. A practical scale. Lancet $1974,2,81-84$

Trentz O (1993): Management des Mehrfachverletzten. Ther Umsch 50: 491-499

Trepesch D, Zollner C, Schmöller G, Ruppert M (2001): Gefahren an der Einsatzstelle Betrachtungen für den Rettungsdienst. Notfall Rettungsmed 4: 505-510

Trunkey D (1991): Initial treatment of patients with extensive trauma .

N Engl J Med 324: 1259-1263

Trupka A, Waydhas C, Nast-Kolb D, Schweiberer L (1995): Der Einfluss der Frühintubation auf die Reduktion des posttraumatischen Organversagens. Unfallchirurg 98: 111-117 
Tscherne H, Regel G : Die Gesamtversorgung des polytraumatisierten Patienten.

In : „Unfallchirurgie in Deutschland Bilanz und Perspektiven“, Hrsg. Ostern HJ, Probst J; Springer-Verlag, Berlin, Heidelberg, New York 1997, 217-221

Tscherne H, Oestern HJ, Sturm JA (1984): Die Belastbarkeit Mehrfachverletzter und ihre Bedeutung für die operative Versorgung.

Langenbecks Arch Chir 364: 71-77

Tscherne H, Regel G, Sturm JA (1987): Schweregrad und Prioritäten bei Mehrfachverletzungen. Chirurg 58: 631-640

Tscherne H, Nerlich ML, Sturm JA (1988): Der Schwerverletzte Patient - Prioritäten und Management. Hefte Unfallheilkd 4: 394-410

Tuttle MS, Smith WR, Williams AE, Agudelo JF, Hartshorn CJ, Moore EE, Morgan SJ (2009): Safety and Efficacy of Damage Control External Fixation Versus Early Definitive Stabilization for femoral Shaft Fractures in the Multiple-Injured Patient. The Journal of Trauma 67: 602-605

Varney $M$, Becker $H$, Röher HD : Therapiekostenermittlung bei polytraumatisierten Patienten während der erststationären Behandlung zur Vereinbarung eines Sonderentgeltes. In: Effektivität und Ökonomie chirurgischen Handeln. Hrsg. Neugebauer E, Troidl H; Thieme-Verlag, Stuttgart 1994, 140-146.

Walcher F (2003): Präklinische Sonographie. Notfall Rettungsmed 6: 476-488

Weninger P, Trimmel H, Nau T, Aldrian S, König F, Vescei V (2005): Polytrauma und Luftrettung Eine retrospektive Analyse der Versorgung im Osten Österreichs am Beispiel eines urbanen Traumazentrums. Unfallchirurg 108: 559-566

Whitesides TE, Heckman MM (1996): Acute Compartment Syndrom : Update on Diagnosis and Treatment. J Am Acta Orthop Surg 4: 209-218

Wieken K : Die schriftliche Befragung In: Techniken der empirischen Sozialforschung, Band 4 : Erhebungsmethoden : Die Befragung. Hrsg. Von J.v.Koolwijk und M. WiekenMayser; Oldenbourg-Verlag, München 1974, 32-41

Wolter D, Wenzl ME, Neikes M : Physikalische Therapie und medizinische Rehabilitation. Kap.33 In : „Unfallchirurgie in Deutschland Bilanz und Perspektiven” Hrsg. Oestern HJ, Probst J; Springer-Verlag, Berlin, Heidelberg, New York 1997, 587-592

Woltmann A, Bühren V (2007): Das Stufenkonzept der Polytraumaversorgung. Intensivmedizin 44: 498-506

Woltmann A, Hamann L, Ulmer AJ, Gerdes J, Bruch HP, Rietschel ET (1998): Molecular mechanism of sepsis. Langenbeck's Arch Surg 383: 2-10

www.traumeregister.de : DGU-Jahresbericht 2000

Zangger P (1989): Die Rehabilitation von polytraumatisierten hirnverletzten Patienten. Ther Umsch 46: 455-459

Zenker W, Havemann D, Besch L (1992): Verletzungsmuster - Leitlinie bei der Beurteilung des Mehrfachverletzten. Unfallchirurgie 18: 69-74 
Ziegenfuß T (1996): Erstversorgung des Polytraumatisierten

Zentralbl Chir 121: 924-942

Zink W, Völkl A, Martin E, Gries A (2002): Die "INTECH" - Studiengruppe: Invasive Notfalltechniken - Ein Ausbildungskonzept in der Notfallmedizin ?

Anaesthesist 51: 853-862 


\section{ABKÜRZUNGSVERZEICHNIS}

ADAC - Allgemeiner Deutscher Automobil Club

AIS - Abbreviated Injury Score

ANV - akutes Nierenversagen

AO - Arbeitsgemeinschaft für Osteosynthesefragen

ARDS - Acute Respiratory Distress Syndrom

Art. - Arterie

ASR - Achillessehnenreflex

BMI - Bodymassindex

bzw. - beziehungsweise

CPAP - Continuous Positive Airway Pressure

DC - Damage Control

DCO - Damage Control Orthopädics

DGU - Deutsche Gesellschaft für Unfallchirurgie

d.h. - das heißt

DIC - disseminierte intravasale Gerinnung

et al. - und andere

ETC - Early-total-care

FA - Facharzt/Fachärztin

GCS - Glasgow Coma Scale

Gr. - Körpergröße

$\mathrm{Hb}$ - Hämoglobin

Hkt. - Hämatokrit

HZV - Herzzeitvolumen

ISS - Injury Severity Score

KG - Körpergewicht

$\mathrm{kg}$ - Kilogramm

Ltd. - leitender

$\mathrm{m}$ - Meter

MESS - Mangled Extremity Severity Score

Min. - Minute

Mio. - Millionen

MODS - Multiorgandysfunktionssyndrom

MOV - Multiorganversagen

Mrd. - Milliarden

NAW - Notarztwagen

NEF - Notarzteinsatzfahrzeug

OP - Operation

PEEP - Positive End-Exspiratory Pressure

PSR - Patellarsehnenreflex

PTS - Hannoverscher Polytrauma-Schlüssel

REHA/AHB - Anschlussheilbehandlung

RTH - Rettungshubschrauber

RTW - Rettungswagen

SIRS - Systemisches inflammatorisches Response Syndrom

u.a. - unter anderem

v.Chr. - vor Christus

WHO - Weltgesundheitsorganisation

z.B. - zum Beispiel

Z.n. - Zustand nach 


\section{DANKSAGUNGEN}

Mein ganz besonderer Dank gilt Herrn Prof.Dr.med.K.Dresing für die Überlassung des interessanten Themas sowie die zahlreichen Anregungen und die Unterstützung während der Durchführung der Arbeit.

Ein weiterer Dank gilt der Abteilung Medizinische Statistik für die beratende Hilfestellung bei der statistischen Analyse der erfassten Daten.

Abschließend spreche ich PD Dr.med.C.Dumont meinen Dank für die Unterstützung bei der Klassifikation der Femurfrakturen aus. 